\title{
Topics on Hamiltonian Dynamics Related to Symbols of Certain Schrodinger Operators Associated with Generators of Levy Processes
}

Rhind, Elian O.T.

How to cite:

Rhind, Elian O.T. (2018) Topics on Hamiltonian Dynamics Related to Symbols of Certain Schrodinger Operators Associated with Generators of Levy Processes. Doctoral thesis, Swansea University.

http://cronfa.swan.ac.uk/Record/cronfa40877

Use policy:

This item is brought to you by Swansea University. Any person downloading material is agreeing to abide by the terms of the repository licence: copies of full text items may be used or reproduced in any format or medium, without prior permission for personal research or study, educational or non-commercial purposes only. The copyright for any work remains with the original author unless otherwise specified. The full-text must not be sold in any format or medium without the formal permission of the copyright holder. Permission for multiple reproductions should be obtained from the original author.

Authors are personally responsible for adhering to copyright and publisher restrictions when uploading content to the repository.

Please link to the metadata record in the Swansea University repository, Cronfa (link given in the citation reference above.) 


\title{
Topics on Hamiltonian Dynamics Related to Symbols of Certain Schrödinger Operators Associated with Generators of Lévy Processes
}

\author{
Elian Osian Tomos Rhind
}

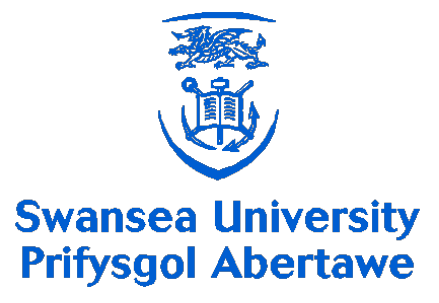

Submitted to Swansea University in the fulfillment of the requirements for the Degree of Doctor of Philosophy

\author{
Department of Mathematics \\ Swansea University \\ 2018
}




\section{Abstract}

Lévy processes give rise to positivity preserving one-parameter operator semigroups, a source for many studies. They are completely characterised by their characteristic exponent $\psi$ which is continuous and negative definite. Using Fourier analysis it can be found that $\psi$ also characterises the corresponding semigroup and its generator.

An interesting case is where $e^{-t \psi} \in L^{1}\left(\mathbb{R}^{n}\right)$ for all $t>0$. This allows us to represent the semigroup as a convolution operator. Through this transition densities are introduced, who are greatly relevant in research associated with probability theory.

As a continuous negative definite function, $\psi$ can define a metric $d_{\psi}$ on $\mathbb{R}^{n}$ which under natural conditions generates the Euclidean topology. This property also leads to looking at metric measure spaces associated with $d_{\psi}$. A key observation is that the diagonal term of the transition density is entirely controlled by the volume of the balls induced by $d_{\psi}$. An idea was to estimate the off-diagonal transition density by a further metric. This raises questions about the convexities of the balls, the dependence of isotropy on parameters, etc.

There is also some interest in the spectral theory of some operators, such as Schrödinger operators associated with $\psi$. The introduction of micro-local analysis induced some development in the spectral theory of (pseudo-) differential operators. From this came an idea of considering the symbol of a (pseudo-) differential operator as a function on the co-tangent bundle. In the case the operator admits a principal part these studies suggest to look at the symbol of the principal part as a Hamilton function. The study of the corresponding dynamics then gives further information about the original pseudo-differential operator, for example its spectral properties.

The aim of this thesis is to start to systematically find out which of the techniques and results from micro-local analysis and the theory of metric measure spaces can be extended to pseudo-differential operators with negative definite symbols generating Feller or $L^{2}$-sub-Markovian semigroups. Through this we would like to provide tools for further studies of such pseudo-differential operators.

To achieve this we concentrate on operators of the form of a Schrödinger operator. In particular, we look at operators $-\psi(D)+V(x)$, with Hamilton function

$$
\mathcal{H}(q, p)=\psi(p)+V(q)
$$


for a suitable potential $V$. Since Hamilton's principle is a central tool we need also study the Lagrange function corresponding to $\mathcal{H}$. The semi-classical asymptotics of classical Schrödinger operators needs a study of the corresponding action function. Hence introducing the Lagrange function leads smoothly into this interest to find the corresponding action function to $\mathcal{H}$.

To accomplish all of this some understanding about convexity as well as negative definite functions is needed and so we collect this required theory in the first two chapters of the thesis. The dynamics associated with $\mathcal{H}$, including the corresponding action function, is then developed in the third chapter.

In some cases, it would be useful to have rather explicit solutions which require concrete potentials. Hence in the fourth chapter we also suggest substitutions for well-studied examples in classical mechanics to provide explicit formulae that could benefit further investigations.

The final two chapters extends the dynamics by taking ideas from the HamiltonJacobi theory. This is possible using the action function associated to $\mathcal{H}$. It provides an equivalent way of studying the dynamics and in some cases, an arguably more efficient way of doing so. 


\section{DECLARATION}

This work has not previously been accepted in substance for any degree and is not being concurrently submitted in candidature for any degree.

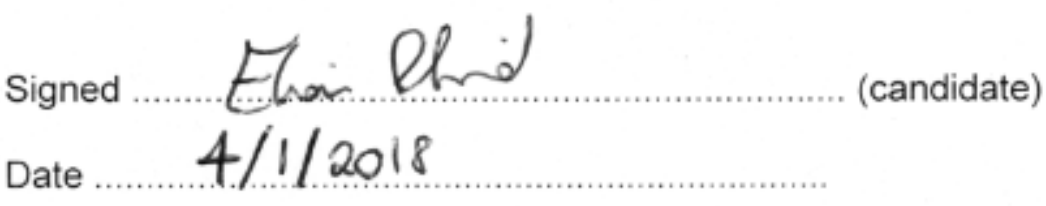

\section{STATEMENT 1}

This thesis is the result of my own investigations, except where otherwise stated.

Other sources are acknowledged by footnotes giving explicit references. A bibliography is appended.

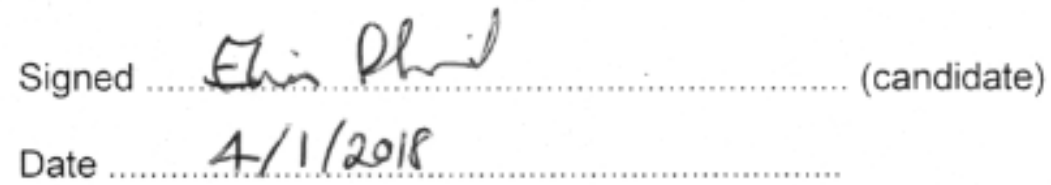

\section{STATEMENT 2}

I hereby give consent for my thesis, if accepted, to be available for photocopying and for inter-library loan, and for the title and summary to be made available to outside organisations.

Signed ....... Elim (candidate)

Date ..........1/2018 


\section{Acknowledgements}

Firstly I would like to express my gratitude to my supervisor Professor Niels Jacob. His everlasting support and patience has been vital in the completion of this thesis. He was always ready to help in any way and his vast array of knowledge knows no end. In fact my thanks extends beyond the duration of my PhD. I have been fortunate to study Mathematics at Swansea University for the past seven years or so starting as an undergraduate leading up to the end of my time as a postgraduate. Professor Jacob has taught me in each of these years, including in the role of my supervisor for my MMath project. It has been a blessing to have had his guidance and these are times I will always value.

I would also like to thank the Mathematics department at Swansea University. The community of staff and students here have always made me feel at home thanks to their prolonging friendliness and encouragement in what I do. In particular I am appreciative of Dr.Andrew Neate for his part in this thesis. His lecture notes on relevant topics were of great help and most crucial in carrying out my studies. I would also like to thank Dr.Gibin Powathil for his valuable aid in instructing me about Matlab which have helped me construct most of the figures one finds in this document. I am grateful to both of these members of staff for their time and efforts in helping me carry out the research.

My time at Swansea has developed myself as a person and has provided many rewarding experiences such as helping on open days as well as being a part of the Further Mathematics programme at Swansea. I am grateful for the help and support from everyone associated with these activities. My time at Swansea has been a pleasurable one which I will always look back on fondly.

My appreciation continues to events that extend externally from the department. Thanks to the department I was able to attend many interesting seminars and colloquia, including those at Gregynog. In addition to this I would like to mention Professor René Schilling and Professor Ingo Witt and express my gratitude for allowing me to attend their workshop at Dresden and Göttingen University respectively. They were enriching experiences from both a personal and mathematical point of view and it was delightful to visit such beautiful locations.

I would like to thank my loving family for always being there for me. They always 
lent an ear for listening throughout the more challenging times for me and I owe it to their never ending encouragement and support in being able to do what I do. Last but not least I would like to mention my friends and colleagues whose company and aid I always value and I thank them for providing a happy environment for me in and out of the working office. 


\section{Contents}

$\begin{array}{ll}\text { Introduction } & 1\end{array}$

$\begin{array}{lr}\text { Index of Notation } & 8\end{array}$

1 Convex Analysis and the Legendre $\begin{array}{lr}\text { Transform } & 10\end{array}$

2 Continuous Negative Definite Functions 25

3 Hamilton and Lagrange Functions Associated with Negative Defi$\begin{array}{lr}\text { nite Functions } & 34\end{array}$

3.1 Revising Classical Mechanics . . . . . . . . . . . . . . . . . . 34

3.2 Hamilton's and Lagrange's Equations Associated with some Negative Definite Symbols . . . . . . . . . . . . . . . . . 40

3.3 Conservation Laws . . . . . . . . . . . . . . . . . . . . . 45

3.4 Relations to the Calculus of Variations . . . . . . . . . . . . . . 52

4 Examples of Hamilton and Lagrange Functions 56

4.1 The Harmonic Oscillator . . . . . . . . . . . . . . . . . . . . . 58

4.2 The Kepler Problem . . . . . . . . . . . . . . . . . . . . . . . . . 81

4.3 Relativistic Dynamics . . . . . . . . . . . . . . . . . . . 104

5 Hamilton-Jacobi Theory $\quad \mathbf{1 2 0}$

5.1 Generating Functions and Canonical Transformations . . . . . . . . . 121

5.2 The Hamilton-Jacobi Equation . . . . . . . . . . . . . . . . . 128

6 Examples Using the Hamilton-Jacobi Theory 137

6.1 Harmonic Oscillator and Kepler Problem

(Hamilton-Jacobi Approach) . . . . . . . . . . . . . . . . . . . . . . . 138

6.2 Relativistic Dynamics

(Hamilton-Jacobi Approach) . . . . . . . . . . . . . . . . . . . . . 148

6.3 Relativistic Dynamics with a Fractional Power . . . . . . . . . . . . 153 
$\begin{array}{ll}\text { A Hypergeometric Series } & 161\end{array}$

B Full Calculations of Examples $\quad 164$ 


\section{Introduction}

Lévy processes, i.e. stochastic processes with stationary and independent increments, form an important class of Markov processes of theoretical interest which has a lot of rather concrete applications, we refer just to [5] and [56]. They give rise to positivity preserving one-parameter operator semigroups, hence to analytic objects, and these semigroups as well as their generators are worth studying by their own right. Lévy processes are completely characterised by their characteristic exponent $\psi: \mathbb{R}^{n} \longrightarrow \mathbb{C}$ which is a continuous function admitting a Lévy-Khinchine representation. Such functions are so called continuous negative definite functions. Using Fourier analysis we see that $\psi$ characterises the corresponding semigroup $\left(T_{t}\right)_{t \geq 0}$ and its generator $A$. The basic formulae are

$$
\begin{gathered}
\mathbb{E}\left(e^{-i\left\langle\xi, X_{t}\right\rangle}\right)=e^{-t \psi(\xi)}, \\
\left(T_{t} u\right)(x)=(2 \pi)^{-\frac{n}{2}} \int_{\mathbb{R}^{n}} e^{i x \cdot \xi} e^{-t \psi(\xi)} \hat{u}(\xi) \mathrm{d} \xi
\end{gathered}
$$

and

$$
A u(x)=-\psi(D) u(x)=-(2 \pi)^{-\frac{n}{2}} \int_{\mathbb{R}^{n}} e^{i x \cdot \xi} \psi(\xi) \hat{u}(\xi) \mathrm{d} \xi
$$

where in (2) and (3) we assume for the moment $u \in \mathcal{S}\left(\mathbb{R}^{n}\right)$. We are here only interested in the case where $e^{-t \psi} \in L^{1}\left(\mathbb{R}^{n}\right)$ for all $t>0$ which allows us to represent $T_{t}$ as a convolution operator

$$
T_{t} u(x)=\int_{\mathbb{R}^{n}} p_{t}(x-y) u(y) \mathrm{d} y
$$

with

$$
p_{t}(x)=(2 \pi)^{-n} \int_{\mathbb{R}^{n}} e^{i x \cdot \xi} e^{-t \psi(\xi)} \mathrm{d} \xi .
$$

Moreover, in this thesis we will always assume $\psi$ to be real-valued which entails $T_{t}$ to be symmetric on $L^{2}\left(\mathbb{R}^{n}\right)$. The family $p_{t}(),. t>0$, consists of the transition densities of the process $\left(X_{t}\right)_{t>0}$ and it is of great relevance in probability theory. For this reason it is an object of intensive studies. We refer here to [5], [56], [6], [7] and 
the references given therein. In particular we want to mention that many of these references refer to even more general processes, for example we mention the work of the Wroclaw School around K.Bogdan, the Seattle School around Z-Q.Chen, or the work of T.Kumagai, A.Kulik and V.Knopova, and many others.

In the late 1970ties to the mid 1990ties Schrödinger operators of type

$$
\psi(D) u+V u
$$

were studied, in particular their spectral theory. We refer to [33], [12], [13]-[15] and in a more wider context [16] and the monograph [17]. The approach was basically a functional analytic one however the Feynman-Kac formula was a much used tool, in particular in [16] and [17].

Spectral theory for (pseudo-) differential operators changed however its face with the advent of micro-local analysis. We mention as principal contributors only L.Hörmander, V.P.Maslov, C.Fefferman, V.Ivrii and M.Shubin (which is by no means a complete list) and refer to [38]-[41], [42] or [61] and the references given there. The idea is to consider the symbol of a (pseudo-) differential operator as a function on the co-tangent bundle and to study this function in more detail in its interaction with geometric constraints as well as data or potentials (given as functions). In the case that the operator admits a principal part these studies suggest to look at the symbol of the principal part also as a Hamilton function and the study of the corresponding dynamics gives further information about the original pseudo-differential operator. Note that in [53] V.P.Maslov suggested to use the full symbol for such an analysis.

Not quite independent of these investigations has been a second development initiated by the papers [37] and [55]. For second-order degenerate elliptic operators $L$ it was possible to introduce certain metrics either by using corresponding vector fields [54] or with the help of the symbol [21] and [22]. In these metrics the fundamental solution of $L$ and the corresponding heat kernel associated with $\frac{\partial}{\partial t}-L$ admit very precise and geometric meaningful estimates. This led first to sub-Riemannian geometry, see [63] or [11] and then to the theory of metric measure spaces associated with local Dirichlet forms, we refer to [28] and [29], and [65]-[67]. Some ideas were also transferred to certain non-local Dirichlet spaces.

The present thesis is embedded into the programme initiated ca. 10 years ago by the supervisor and this programme tries systematically to find out which of the techniques and results from micro-local analysis and the theory of metric measure spaces can be extended or generalised to pseudo-differential operators with negative 
definite symbols generating Feller or $L^{2}$-sub-Markovian semigroups. These operators are of type

$$
-q(x, D) u(x)=-(2 \pi)^{-\frac{n}{2}} \int_{\mathbb{R}^{n}} e^{i x \cdot \xi} q(x, \xi) \hat{u}(\xi) \mathrm{d} \xi, \quad u \in \mathcal{S}\left(\mathbb{R}^{n}\right),
$$

where $q: \mathbb{R}^{n} \times \mathbb{R}^{n} \longrightarrow \mathbb{C}$ is a continuous function such that $\xi \mapsto q(x, \xi)$ is negative definite, i.e. admits for every fixed $x \in \mathbb{R}^{n}$ a Lévy-Khinchine representation. Such operators were studied systematically since the late 1980ties, we refer to the monographs [43]-[45] and [7] for overviews in which in particular the seminal existences results W.Hoh [34]-[36] and the equally important papers [57]-[59] of R.Schilling on the probabilistic relevance of the symbol are discussed. The basic problem in the theory is that in general these symbols do not fit into any of the framework discussed above.

An important fact is that if $\psi: \mathbb{R}^{n} \longrightarrow \mathbb{R}$ is a continuous negative definite function such that $\psi(\xi)=0$ if and only if $\xi=0$, then

$$
d_{\psi}(\xi, \eta):=\psi^{\frac{1}{2}}(\xi-\eta)
$$

defines a metric on $\mathbb{R}^{n}$ which under natural conditions generates the Euclidean topology, see [48]. One of the key observations in [48] based on [50] was that the diagonal term of $p_{t}(x-y)$, i.e. $p_{t}(0)$, is then entirely controlled by the volume of the balls $B_{d_{\psi}}$. A further idea in [48] was to try to estimate $p_{t}(x)$ off-diagonal, i.e. for $x \neq 0$, by a metric $\delta_{t, \psi}$ to obtain estimates of the type

$$
p_{t}(x-y) \asymp p_{t}(0) e^{-\delta_{t, \psi}^{2}(x, y)} .
$$

Examples were provided in [48] and the idea was developed further in [9], see also [8] and the forthcoming paper [20]. In [31], see also [30], [52], and [49], the metric $d_{\psi}$ was investigated in more detail, questions of interest are the convexities of balls, the dependence of isotropy on parameters etc. In this thesis the main interest is to provide tools for further studies of pseudo-differential operators of type (7) and we concentrate eventually on operators of the form (6). The main goal is to look at the Hamilton function

$$
\mathcal{H}(q, p)=\psi(p)+V(q)
$$

for a suitable potential $V$. Since Hamilton's principle is a central tool we need also to study the Lagrange function corresponding to $\mathcal{H}$ and it took some effort to develop the appropriate frame. The first observation is that $\psi$ need not to be convex or coercive or differentiable, a typical assumption in the classical theory (and easily achievable for differential operators) is that $\mathcal{H}$ is a $C^{2}$-function. In order to make some non-trivial progress we left on the one hand side the classical convexity and 
coercivity assumptions but we reduced the differentiability assumption to $C^{1}$. We also gave up homogeneity assumptions on $\psi$, they would be too limiting. While the continuous negative definite function $\xi \mapsto|\xi|^{\alpha}, 1<\alpha<2$, are obvious examples, we provided with

$$
\psi_{E R}\left(\xi_{1}, \xi_{2}\right)=\left(\left|\xi_{1}\right|^{\alpha_{1}}+\left|\xi_{2}\right|^{\beta_{1}}\right)^{\gamma_{1}}+\left(\left|\xi_{1}\right|^{\alpha_{2}}+\left|\xi_{2}\right|^{\beta_{2}}\right)^{\gamma_{2}}
$$

$1<\alpha_{i}, \beta_{i}<2,0<\gamma_{i}<1, i=1,2$, an example which shows full anisotropy and does not allow us to define a homogeneous principal part, but is still convex, coercive and $C^{1}$. For coercive, convex and continuously differentiable continuous negative definite functions $\psi$ we may now look at their Legendre transforms $\psi^{*}$, i.e. their convex dual functions. By looking at appropriate potentials we can also work with the partial Legendre transform of $\psi(p)+V(q)$ (transforming the variable $p$ ) and by this we obtain the corresponding Lagrange function. Typically we find under suitable assumptions

$$
\mathcal{L}(q, \eta)=\psi^{*}(\eta)-V(q)
$$

and latest at this stage we leave "classical" mechanics. We now turn to the main question of this thesis namely to develop for the case where $\psi$ is a suitable continuous negative definite function the dynamics generated by $\mathcal{H}$ given by (10) with corresponding Lagrange function $\mathcal{L}$ given by (12). The aim was in particular to get in some cases rather explicit solutions which require concrete potentials. We make the suggestion to study as a substitute of the classical harmonic oscillator the Hamilton function

$$
\mathcal{H}(q, p)=\psi(p)+\psi^{*}(q)
$$

and for the Hamiltonian extending the Kepler problem we suggest

$$
\mathcal{H}(q, p)=\psi(p)+F^{-1}\left(\frac{1}{\psi(.)}\right)(q) .
$$

Discussing these examples in much detail for

$$
\psi(p)=\frac{1}{\alpha}\|p\|^{\alpha}, \quad 1<\alpha<2,
$$

is a major part of our investigation, not least to provide for further investigations concrete and where possible explicit formulae. Our basic idea in the presentation is to develop along the lines of classical mechanics, see [26] or [27], the modifications needed for the case (12) and then turn in full details to concrete examples.

As was pointed out earlier, in the case of second-order degenerate elliptic differential operators the underlying Hamilton dynamics associated with the principal symbol is an important tool. We would like to add that the semi-classical asymptotics of classical Schrödinger operators needs a study of the corresponding action 
function of the underlying classical system. Thus if we want to study some semiclassical asymptotics of certain Schrödinger operators of type $\psi(D)+V(x)$, we first of all need a "classical" system corresponding to $\psi(D)+V(x)$ and of course we suggest (10) to be this object. Therefore there is also some interest to find, if possible, explicitly the corresponding action function $S$, or at least some of its basic properties. These are indications for further studies which will go beyond our thesis.

We now discuss briefly the context of each chapter. In the first chapter we collect results we will need in regard to convexity. In particular we learn about Legendre transforms and coercivity. Also included here is a result for finding the Legendre transform of rotational invariant functions. An example for when this is useful is when $\psi$ is defined as at (15). The most interesting example introduced will be $\psi_{E R}$ from (11). We prove both its convexity and its coercivity. In regard to finding the Legendre transform of $\psi_{E R}$ we are unable to calculate it explicitly. However with the help of the inf-convolute we are able to provide an upper and lower bound for $\psi_{E R}^{*}$.

The second chapter covers continuous negative definite functions. We provide examples including symbols $\psi$ for $(6)$ and we discuss the metric $d_{\psi}$ from (8). In particular we consider the metric measure space $\left(\mathbb{R}^{2}, d_{\psi_{E R}}, \lambda^{(2)}\right)$. The relation of continuous negative definite functions to convolution semigroups is presented here as well as their Lévy-Khinchine representation. To expand our portfolio of examples for $\psi$ we also introduce Bernstein functions. It is found that the composition of a continuous negative definite function with a Bernstein function is also continuous negative definite and hence this induces $\psi_{E R}$ amongst other examples to be continuous and negative definite.

We introduce the dynamics in the third chapter. The first section is a revision of elements of classical mechanics that we will use when developing the dynamics of (10). If anything this initial part acts as a checklist for when we attempt to transfer each property to the case of pseudo-differential operators. The second section begins to set the problem of studying the dynamics associated with (10). By studying the dynamics we mean to solve a system of first-order ordinary differential equations known as Hamilton's equations. We find also the corresponding Lagrangian using the partial Legendre transform introduced in the first chapter as well as proving the equivalence of Hamilton's equations to a system of second-order ordinary differential equations known as Lagrange's equations. It is here where care in regard to the smoothness of the symbols becomes especially crucial. The third section introduces conservation laws to our study which help simplify the solving of Hamilton's and Lagrange's equations. We already know Hamilton's principle to be important and so to close the chapter some ideas of the calculus of variations are provided where the action function is taken to have the Lagrangian from (12).

In the fourth chapter we study the dynamics associated to some examples of (10). 
As mentioned before the idea here is to choose examples with concrete potentials who act as analogues to well-studied problems in classical mechanics. Two of the suggested examples we have already introduced at (13) and (14). We also suggest a third example given by

$$
\mathcal{H}(q, p)=\sqrt{m^{2}+\|p\|^{2}}-m+V(q), \quad m>0,
$$

which corresponds to the Hamiltonian for a type of relativistic dynamics.

As mentioned before, we devote much detail to discuss the dynamics corresponding to (13) and (14) with $\psi(\xi)$ given by (15). We discuss both, the Hamiltonian system and the Lagrange system, of course our examples for the Harmonic Oscillator and Kepler problem may be viewed as generalisations of their corresponding Hamiltonians in classical mechanics. We will show how the "classical" case may be obtained as special cases in either example. We also demonstrate some "classical" properties of (13) and (14). For example our Kepler problem in three dimensions, i.e. setting $n=3$, may be reduced to an equivalent two-dimensional problem, similar to what can be done with the Kepler problem in classical mechanics.

The solutions we obtain when solving Hamilton's or Lagrange's equations are not always explicit. Some may be determined by certain special functions such as hypergeometric series but others leave integrals who are difficult to compute. Hence we also provide in this chapter figures demonstrating the behaviour of the solutions so that we may learn more about them. These figures were constructed using Mathematica and Matlab. Due to the length of the calculations behind these examples we do not provide them at their fullest in this chapter. Instead we collect the main results and focus on their analysis. We reserve the full details of the workings for an appendix towards the end of thesis.

Next we seek more efficient ways to study the dynamics. In this fifth chapter we do this by developing an analogue to the Hamilton-Jacobi theory. The idea is to transform the variables $q$ and $p$ so that the solving of Hamilton's equations is facilitated. In doing this we obtain the Hamilton-Jacobi equation which is a firstorder partial differential equation. This provides an equivalent approach to solving Hamilton's equations. Developing this theory requires the action function $S$ hence the conclusive section of the third chapter will be of most use here. Of course, this is a transfer of classical results.

The final chapter follows similarly in style to the fourth chapter and picks up on the examples found there. However this time they are investigated using the Hamilton-Jacobi theory developed in the previous chapter. Of course we expect to obtain results comparable to those obtained before. An additional example for the relativistic dynamics is also considered here. It in some sense acts as a generalisation to (16) which was considered in the fourth chapter.

Appendix A collects material about hypergeometric series and as mentioned 
before, Appendix B gives further details to some lengthy calculations needed in discussing the examples. 


\section{Index of Notation}

$\mathbb{N}$

$\mathbb{R}$

$\mathbb{R}_{+}$

$\mathbb{R}^{n}$

$\mathbb{C}$

$\bar{A}$

int $A$

$\operatorname{det} M$

$x \cdot y=\langle x, y\rangle$

$\left(u_{n}\right)_{n \in \mathbb{N}}$

$\left.u\right|_{G}$

$\operatorname{Re} u$

$\operatorname{dom} u$

epi $u$

Hess $u$

$\mathrm{d} u$

$\nabla u$

$u^{*}$

$u_{1} \circ u_{2}$

$u_{1} \square u_{2}$

$F u=\hat{u}$

$F^{-1} u$

$\Gamma(n)$

${ }_{2} F_{1}(a, b ; c ; z)$

$\mathcal{H}$

$\mathcal{L}$

$\epsilon_{a}$

$\lambda^{(n)}$

$\mathcal{B}^{(n)}(G)$

$(\Omega, \mathcal{A})$ set of natural numbers

set of real numbers

set of positive real numbers

$n$-dimensional Euclidean space

set of complex numbers

closure of a set $A$

interior of a set $A$

determinant of matrix $M$

dot or scalar product of $x$ and $y$

sequence of functions $u_{n}$

a function $u$ restricted to $G$

real part of a function $u$

domain of a function $u$

epigraph of a function $u$

Hessian of a function $u$

differential of a function $u$

gradient of a function $u$

Legendre transform of a function $u$

composition of functions $u_{1}$ and $u_{2}$

inf-convolute of functions $u_{1}$ and $u_{2}$

Fourier transform of $u$

inverse Fourier transform of $u$

Gamma function of $n$

hypergeometric series with parameters $a, b$ and $c$, and complex variable $z$

Hamilton function

Lagrange function

Dirac measure at $a \in \mathbb{R}^{n}$

$n$-dimensional Lebesgue measure

$n$-dimensional Borel sets of the set $G$

measurable space with set $\Omega$ and $\sigma$-field $\mathcal{A}$ 


$$
(\Omega, \mathcal{A}, \mu)
$$$$
\mathcal{A}_{1} \otimes \mathcal{A}_{2}
$$$$
\mu_{1} \otimes \mu_{2}
$$$$
\left(\Omega_{1} \otimes \Omega_{2}, \mathcal{A}_{1} \otimes \mathcal{A}_{2}, \mu_{1} \otimes \mu_{2}\right)
$$

$\left(\mu_{t}\right)_{t \geq 0}$
$C(G)$
$C_{c}(G)$
$C^{m}(G)$
$C^{m}\left(G ; \mathbb{R}^{n}\right)$
$C^{\infty}(G)$
$C^{\infty}\left(G ; \mathbb{R}^{n}\right)$
$C_{c}^{\infty}\left(G ; \mathbb{R}^{n}\right)$

$\mathcal{S}^{\prime}\left(\mathbb{R}^{n}\right)$

$N\left(\mathbb{R}^{n}\right)$

$C N\left(\mathbb{R}^{n}\right)$

$\psi(D)$

$d_{\psi}$

$(X, d)$

$(X, d, \mu)$

$B_{r}^{d}(x)$

$K_{r}^{d}(x)$

$|x|$

$\|x\|$

$\|x\|_{p}$

$\|u\|_{C^{1}(a, b)}$ meausre space with measurable space $(\Omega, \mathcal{A})$ and measure $\mu$

product $\sigma$-field of $\sigma$-fields $\mathcal{A}_{1}$ and $\mathcal{A}_{2}$

product measure of measures $\mu_{1}$ and $\mu_{2}$

product measure space of measure spaces $\left(\Omega_{1}, \mathcal{A}_{1}, \mu_{1}\right)$ and $\left(\Omega_{2}, \mathcal{A}_{2}, \mu_{2}\right)$

convolution semigroup of measures $\mu_{t}$

continuous functions on $G$

continuous functions with compact support on $G$ $m$-times continuously differentiable functions on $G$ $\mathbb{R}^{n}$-valued $m$-times continuously differentiable functions on $G$

arbitrarily differentiable functions on $G$

$\mathbb{R}^{n}$-valued arbitrarily differentiable functions on $G$

$\mathbb{R}^{n}$-valued arbitrarily differentiable functions with compact support on $G$

space of tempered distributions on $\mathbb{R}^{n}$

space of negative definite functions on $\mathbb{R}^{n}$

space of continuous negative definite functions on $\mathbb{R}^{n}$

pseudo-differential operator with symbol $\psi(\xi)$ metric generated by $\psi \in C N\left(\mathbb{R}^{n}\right)$

metric space with metric $d$ defined on $X \times X$

metric measure space with metric space $(X, d)$ and measure $\mu$

open ball of radius $r$ and centre $x$ induced by a metric $d$

closed ball of radius $r$ and centre $x$ induced by a metric $d$

Euclidean norm in $\mathbb{R}$

Euclidean norm in $\mathbb{R}^{n}$

$p$-norm in $\mathbb{R}^{n}, p \geq 1$

$C^{1}$-norm of a continuously differentiable function $u$ defined on $(a, b)$ 


\section{Chapter 1}

\section{Convex Analysis and the Legendre Transform}

Here we collect some results we will need from convex analysis. This will be done following mainly Chapter II of Ekeland's book [19] unless stated otherwise.

Consider the function $F: \mathbb{R}^{n} \longrightarrow \mathbb{R} \cup\{+\infty\}$. By the domain of $F$ we mean those points $x \in \mathbb{R}^{n}$ such that the values $F(x)$ are finite:

$$
\operatorname{dom} F:=\left\{x \in \mathbb{R}^{n} \mid F(x)<+\infty\right\} .
$$

The epigraph of $F$ is the following subset of $\mathbb{R}^{n} \times \mathbb{R}$ :

$$
\text { epi } F:=\left\{(x, a) \in \mathbb{R}^{n} \times \mathbb{R} \mid F(x) \leq a\right\} \text {. }
$$

The function $F$ will be referred to as proper if it is not identically $+\infty$. Comparing (1.1) to (1.2) this is equivalent to saying that the domain of $F$ is non-empty.

In general in this chapter we will consider functions $F$ who may or may not have values of $+\infty$. When calling such functions "proper" we will attain the notation of $F: \mathbb{R}^{n} \longrightarrow \mathbb{R} \cup\{+\infty\}$, even though the functions do have values in $\{+\infty\}$. This notation will leave the majority of the thesis unaffected as most of the functions we will consider following this chapter are proper where $\{+\infty\}$ is not included in their range. The only exception can be found at Section 4.3 when discussing examples for the relativistic dynamics.

Definition 1.1. The function $F: \mathbb{R}^{n} \longrightarrow \mathbb{R} \cup\{+\infty\}$ is convex if epi $F \subset \mathbb{R}^{n} \times \mathbb{R}$ is a convex set.

A further equivalent condition for convexity is given below: 
Proposition 1.2. The function $F: \mathbb{R}^{n} \longrightarrow \mathbb{R} \cup\{+\infty\}$ is convex if and only if for all $x, y \in \mathbb{R}^{n}$ and $\lambda, \mu \in \mathbb{R}_{+}$it holds that

$$
F(\lambda x+\mu y) \leq \lambda F(x)+\mu F(y)
$$

where $\lambda+\mu=1$.

In fact when $F$ is twice continuously differentiable there also exists the following characterisation for convexity:

Theorem 1.3. (see [47, pages 272 to 273])

Consider a proper function $F \in C^{2}\left(\mathbb{R}^{n}\right)$. Then $F$ is convex if and only if

$$
\langle\operatorname{Hess} F(x) y, y\rangle=\sum_{k, l=1}^{n} \frac{\partial^{2} F}{\partial x_{k} \partial x_{l}}(x) y_{k} y_{l} \geq 0 \quad \text { for all } \quad x, y \in \mathbb{R}^{n},
$$

i.e. the Hesse matrix of $F$, denoted Hess $F$, is positive semi-definite.

We will also need the notion of a lower semicontinuous function on $\mathbb{R}^{n}$ :

Definition 1.4. The function $F: \mathbb{R}^{n} \longrightarrow \mathbb{R} \cup\{+\infty\}$ is said to be lower semicontinuous (l.s.c) if epi $F \subset \mathbb{R}^{n} \times \mathbb{R}$ is a closed set.

Equivalently one can also show:

Proposition 1.5. A function $F: \mathbb{R}^{n} \longrightarrow \mathbb{R} \cup\{+\infty\}$ is l.s.c if and only if either of the following conditions are true:

(i) the set $\{x \mid F(x) \leq a\}$ is closed in $\mathbb{R}^{n}$ for every $a \in \mathbb{R}$;

(ii) $\liminf _{y \rightarrow x} F(y) \geq F(x)$.

The continuity of a function implies its lower semicontinuity. However, the converse is not always true:

Example 1.6. The function $\varphi_{G}: G \longrightarrow \mathbb{R} \cup\{+\infty\}, G \subset \mathbb{R}^{n}$, defined by

$$
\varphi_{G}(x):= \begin{cases}0, & \text { if } x \in G, \\ +\infty, & \text { if } x \notin G,\end{cases}
$$

is l.s.c if and only if $G$ is closed. However it is not continuous.

If $F$ is a convex l.s.c function then this implies that $\lambda F$ is convex l.s.c for all $\lambda>0$. Introducing a further convex l.s.c function $H, F+H$ is also convex l.s.c. Overall the set of all convex (l.s.c) functions defined on $\mathbb{R}^{n}$ forms a convex cone.

Also of use is a result from [47, page 256]:

Proposition 1.7. Let $F_{n}: \mathbb{R}^{n} \longrightarrow \mathbb{R} \cup\{+\infty\}$ form a sequence of proper convex functions $\left(F_{n}\right)_{n \in \mathbb{N}^{*}}$. Suppose its limit $F(x):=\lim _{n \rightarrow \infty} F_{n}(x)$ exists and is proper for every $x \in \mathbb{R}^{n}$. Then $F: \mathbb{R}^{n} \longrightarrow \mathbb{R} \cup\{+\infty\}$ is also convex. 


\section{Example 1.8.}

A. The function $x \mapsto \psi_{\alpha}(x):=\frac{1}{\alpha}\|x\|^{\alpha}, 1<\alpha \leq 2$, is proper and convex on its domain $\mathbb{R}^{n}$. Its epigraph is the set

$$
\text { epi } \psi_{\alpha}=\left\{(x, a) \in \mathbb{R}^{n} \times \mathbb{R} \mid \frac{1}{\alpha}\|x\|^{\alpha} \leq a\right\} .
$$

Being continuous $\psi_{\alpha}$ is l.s.c.

$\boldsymbol{B}$. We will return to this example at many instances later. Consider the function $\psi_{E R}: \mathbb{R}^{2} \longrightarrow \mathbb{R}$ defined as

$$
\psi_{E R}\left(\xi_{1}, \xi_{2}\right):=\left(\left|\xi_{1}\right|^{\alpha_{1}}+\left|\xi_{2}\right|^{\beta_{1}}\right)^{\gamma_{1}}+\left(\left|\xi_{1}\right|^{\alpha_{2}}+\left|\xi_{2}\right|^{\beta_{2}}\right)^{\gamma_{2}}
$$

for $1<\alpha_{i}, \beta_{i}<2,0<\gamma_{i}<1, i=1,2$. It is clear that $\psi_{E R}$ is proper for any $\left(\xi_{1}, \xi_{2}\right) \in \mathbb{R}^{2}$. Therefore $\operatorname{dom} \psi_{E R}=\mathbb{R}^{2}$. Following (1.2) the epigraph is a subset of $\mathbb{R}^{2} \times \mathbb{R}$ :

$$
\text { epi } \psi_{E R}:=\left\{\left(\left(\xi_{1}, \xi_{2}\right), a\right) \in \mathbb{R}^{2} \times \mathbb{R} \mid \psi_{E R}\left(\xi_{1}, \xi_{2}\right) \leq a\right\} .
$$

We know that $\psi_{E R}$ is continuous on $\mathbb{R}^{2}$ hence it must also be l.s.c.

Note that $\psi_{E R}$ is not a $C^{2}$-function and therefore we can not use Theorem 1.3 to prove that $\psi_{E R}$ is convex on $\mathbb{R}^{2}$. We claim that for $\alpha_{i} \gamma_{i}>1$ and $\beta_{i} \gamma_{i}>1$ the function $\psi_{E R}$ is convex.

Proof of the convexity of $\psi_{E R}$. For this proof we will require that $\alpha_{i} \gamma_{i}>1$ and $\beta_{i} \gamma_{i}>1$. To begin we recall that the set of convex functions forms a convex cone. Hence for the convexity of (1.3) it is sufficient to prove the convexity of the mappings $\psi_{i}: \mathbb{R}^{2} \longrightarrow \mathbb{R}, i=1,2$, where

$$
\psi_{i}\left(\xi_{1}, \xi_{2}\right):=\left(\left|\xi_{1}\right|^{\alpha_{i}}+\left|\xi_{2}\right|^{\beta_{i}}\right)^{\gamma_{i}}
$$

with each $\alpha_{i}, \beta_{i}$ and $\gamma_{i}$ as in Example 1.8.B.

A problem is that (1.4) is not a $C^{2}$-function hence we cannot use Theorem 1.3. To overcome this we define $\phi_{n}: \mathbb{R}^{2} \longrightarrow \mathbb{R}$ such that

$$
\phi_{n}\left(\xi_{1}, \xi_{2}\right):=\left(\left(\xi_{1}^{2}+\epsilon_{n}\right)^{\frac{\alpha_{i}}{2}}+\left(\xi_{2}^{2}+\epsilon_{n}\right)^{\frac{\beta_{i}}{2}}\right)^{\gamma_{i}}
$$

where $\left(\epsilon_{n}\right)_{n \in \mathbb{N}}$ is a sequence of positive real numbers converging to 0 as $n$ tends to infinity.

Noting that $\phi_{n}$ is $C^{2}$ we will prove its convexity using Theorem 1.3. Having done this, taking the limit $n \rightarrow \infty$ gives $\psi_{i}$, which now being the limit of a sequence of convex functions, we can then conclude $\psi_{E R}$ to be convex. 
We begin with the convexity of $\phi_{n}$. We will need its first and second-order partial derivatives, therefore it will be useful to define

$$
h_{n}\left(\xi_{1}\right):=\left(\xi_{1}^{2}+\epsilon_{n}\right)^{\frac{\alpha_{i}}{2}}
$$

and

$$
g_{n}\left(\xi_{2}\right):=\left(\xi_{2}^{2}+\epsilon_{n}\right)^{\frac{\beta_{i}}{2}}
$$

and calculate their derivatives. The first-order derivatives of $h_{n}$ and $g_{n}$ are

$$
\frac{\mathrm{d} h_{n}}{\mathrm{~d} \xi_{1}}=\alpha_{i} \xi_{1}\left(\xi_{1}^{2}+\epsilon_{n}\right)^{\frac{\alpha_{i}}{2}-1}=\frac{\alpha_{i} \xi_{1} h_{n}\left(\xi_{1}\right)}{\left(\xi_{1}^{2}+\epsilon_{n}\right)}
$$

and

$$
\frac{\mathrm{d} g_{n}}{\mathrm{~d} \xi_{2}}=\beta_{i} \xi_{2}\left(\xi_{2}^{2}+\epsilon_{n}\right)^{\frac{\beta_{i}}{2}-1}=\frac{\beta_{i} \xi_{2} g_{n}\left(\xi_{2}\right)}{\left(\xi_{2}^{2}+\epsilon_{n}\right)}
$$

respectively. The second-order derivatives are

$$
\frac{\mathrm{d}^{2} h_{n}}{\mathrm{~d} \xi_{1}^{2}}=\frac{\alpha_{i}\left(\epsilon_{n}+\left(\alpha_{i}-1\right) \xi_{1}^{2}\right)}{\left(\xi_{1}^{2}+\epsilon_{n}\right)^{2-\frac{\alpha_{i}}{2}}}=\frac{\alpha_{i}\left(\epsilon_{n}+\left(\alpha_{i}-1\right) \xi_{1}^{2}\right) h_{n}\left(\xi_{1}\right)}{\left(\xi_{1}^{2}+\epsilon_{n}\right)^{2}}
$$

and

$$
\frac{\mathrm{d}^{2} g_{n}}{\mathrm{~d} \xi_{2}^{2}}=\frac{\beta_{i}\left(\epsilon_{n}+\left(\beta_{i}-1\right) \xi_{2}^{2}\right)}{\left(\xi_{2}^{2}+\epsilon_{n}\right)^{2-\frac{\beta_{i}}{2}}}=\frac{\beta_{i}\left(\epsilon_{n}+\left(\beta_{i}-1\right) \xi_{2}^{2}\right) g_{n}\left(\xi_{2}\right)}{\left(\xi_{2}^{2}+\epsilon_{n}\right)^{2}} .
$$

Rewriting $\phi_{n}$ as

$$
\phi_{n}\left(\xi_{1}, \xi_{2}\right)=\left(h_{n}\left(\xi_{1}\right)+g_{n}\left(\xi_{2}\right)\right)^{\gamma_{i}},
$$

its first-order partial derivatives follow as

$$
\frac{\partial \phi_{n}}{\partial \xi_{1}}=\gamma_{i} h_{n}^{\prime}\left(\xi_{1}\right)\left(h_{n}\left(\xi_{1}\right)+g_{n}\left(\xi_{2}\right)\right)^{\gamma_{i}-1}
$$

and

$$
\frac{\partial \phi_{n}}{\partial \xi_{2}}=\gamma_{i} g_{n}^{\prime}\left(\xi_{2}\right)\left(h_{n}\left(\xi_{1}\right)+g_{n}\left(\xi_{2}\right)\right)^{\gamma_{i}-1}
$$

This gives

$$
\begin{aligned}
\frac{\partial^{2} \phi_{n}}{\partial \xi_{1}^{2}} & =\frac{\gamma_{i} h_{n}^{\prime \prime}\left(\xi_{1}\right)\left(h_{n}\left(\xi_{1}\right)+g_{n}\left(\xi_{2}\right)\right)+\gamma_{i}\left(\gamma_{i}-1\right) h_{n}^{\prime 2}\left(\xi_{1}\right)}{\left(h_{n}\left(\xi_{1}\right)+g_{n}\left(\xi_{2}\right)\right)^{2-\gamma_{i}}} \\
\frac{\partial^{2} \phi_{n}}{\partial \xi_{2}^{2}} & =\frac{\gamma_{i} g_{n}^{\prime \prime}\left(\xi_{2}\right)\left(h_{n}\left(\xi_{1}\right)+g_{n}\left(\xi_{2}\right)\right)+\gamma_{i}\left(\gamma_{i}-1\right) g_{n}^{\prime 2}\left(\xi_{2}\right)}{\left(h_{n}\left(\xi_{1}\right)+g_{n}\left(\xi_{2}\right)\right)^{2-\gamma_{i}}}
\end{aligned}
$$


and

$$
\frac{\partial^{2} \phi_{n}}{\partial \xi_{1} \partial \xi_{2}}=\gamma_{i}\left(\gamma_{i}-1\right) h_{n}^{\prime}\left(\xi_{1}\right) g_{n}^{\prime}\left(\xi_{2}\right)\left(h_{n}\left(\xi_{1}\right)+g_{n}\left(\xi_{2}\right)\right)^{\gamma_{i}-2}
$$

For its convexity we want to check if the Hessian matrix of $\phi_{n}$ is positive semidefinite:

$$
\begin{aligned}
& H_{\phi_{n}}=\frac{1}{\left(h_{n}\left(\xi_{1}\right)+g_{n}\left(\xi_{2}\right)\right)^{2-\gamma_{i}}} \times \\
& \left(\begin{array}{cc}
\gamma_{i} h_{n}^{\prime \prime}\left(\xi_{1}\right)\left(h_{n}\left(\xi_{1}\right)+g_{n}\left(\xi_{2}\right)\right) & \gamma_{i}\left(\gamma_{i}-1\right) h_{n}^{\prime}\left(\xi_{1}\right) g_{n}^{\prime}\left(\xi_{2}\right) \\
+\gamma_{i}\left(\gamma_{i}-1\right) h_{n}^{\prime 2}\left(\xi_{1}\right) & \gamma_{i} g_{n}^{\prime \prime}\left(\xi_{2}\right)\left(h_{n}\left(\xi_{1}\right)+g_{n}\left(\xi_{2}\right)\right) \\
\gamma_{i}\left(\gamma_{i}-1\right) h_{n}^{\prime}\left(\xi_{1}\right) g_{n}^{\prime}\left(\xi_{2}\right) & +\gamma_{i}\left(\gamma_{i}-1\right) g_{n}^{\prime 2}\left(\xi_{2}\right)
\end{array}\right)
\end{aligned}
$$

Using the theory of positive definite matrices from [62, page 97], to gain positive semi-definitness of $H_{\phi_{n}}$ we require

$$
\begin{aligned}
& \gamma_{i} g_{n}^{\prime \prime}\left(\xi_{2}\right)\left(h_{n}\left(\xi_{1}\right)+g_{n}\left(\xi_{2}\right)\right)+\gamma_{i}\left(\gamma_{i}-1\right) g_{n}^{\prime 2}\left(\xi_{2}\right) \geq 0 \\
& \gamma_{i} h_{n}^{\prime \prime}\left(\xi_{1}\right)\left(h_{n}\left(\xi_{1}\right)+g_{n}\left(\xi_{2}\right)\right)+\gamma_{i}\left(\gamma_{i}-1\right){h_{n}^{\prime}}^{2}\left(\xi_{1}\right) \geq 0
\end{aligned}
$$

and

$$
\operatorname{det} H_{\phi_{n}} \geq 0 .
$$

Note in the above it has been multiplied through by $\left(h_{n}\left(\xi_{1}\right)+g_{n}\left(\xi_{2}\right)\right)^{2-\gamma_{i}}$ to simplify the conditions.

Let us first check (1.10). Note we can write instead

$$
g_{n}^{\prime \prime}\left(\xi_{1}\right)\left(h_{n}\left(\xi_{1}\right)+g_{n}\left(\xi_{2}\right)\right) \geq\left(1-\gamma_{i}\right) g_{n}^{\prime 2}\left(\xi_{2}\right) .
$$

Implementing (1.7) and (1.9) we see that

$$
\frac{\beta_{i}\left(\epsilon_{n}+\left(\beta_{i}-1\right) \xi_{2}^{2}\right)}{\left(\xi_{2}^{2}+\epsilon_{n}\right)^{2-\frac{\beta_{i}}{2}}}\left(\left(\xi_{1}^{2}+\epsilon_{n}\right)^{\frac{\alpha_{i}}{2}}+\left(\xi_{2}^{2}+\epsilon_{n}\right)^{\frac{\beta_{i}}{2}}\right) \geq\left(1-\gamma_{i}\right) \beta_{i}^{2} \xi_{2}^{2}\left(\xi_{2}^{2}+\epsilon_{n}\right)^{\beta_{i}-2}
$$

or

$$
\left(\xi_{2}^{2}+\epsilon_{n}\right)^{-\frac{\beta_{i}}{2}} \beta_{i}\left(\epsilon_{n}+\left(\beta_{i}-1\right) \xi_{2}^{2}\right)\left(\left(\xi_{1}^{2}+\epsilon_{n}\right)^{\frac{\alpha_{i}}{2}}+\left(\xi_{2}^{2}+\epsilon_{n}\right)^{\frac{\beta_{i}}{2}}\right) \geq\left(1-\gamma_{i}\right) \beta_{i}^{2} \xi_{2}^{2}
$$

To see when (1.13) is true consider

$$
\beta_{i}\left(\epsilon_{n}+\left(\beta_{i}-1\right) \xi_{2}^{2}\right) \geq\left(1-\gamma_{i}\right) \beta_{i}^{2} \xi_{2}^{2}
$$

or through some simplification

$$
\epsilon_{n}-\xi_{2}^{2} \geq-\gamma_{i} \beta_{i} \xi_{2}^{2}
$$


This then gives

$$
\xi_{2}^{2}\left(\gamma_{i} \beta_{i}-1\right) \geq-\epsilon_{n}
$$

which is true for $\gamma_{i} \beta_{i}>1$. Noting that

$$
\left(\xi_{2}^{2}+\epsilon_{n}\right)^{-\frac{\beta_{i}}{2}}\left(\left(\xi_{1}^{2}+\epsilon_{n}\right)^{\frac{\alpha_{i}}{2}}+\left(\xi_{2}^{2}+\epsilon_{n}\right)^{\frac{\beta_{i}}{2}}\right)=\frac{\left(\xi_{1}^{2}+\epsilon_{n}\right)^{\frac{\alpha_{i}}{2}}}{\left(\xi_{2}^{2}+\epsilon_{n}\right)^{\frac{\beta_{i}}{2}}}+1>1,
$$

it is clear that

$$
\begin{aligned}
& \left(\xi_{2}^{2}+\epsilon_{n}\right)^{-\frac{\beta_{i}}{2}} \beta_{i}\left(\epsilon_{n}+\left(\beta_{i}-1\right) \xi_{2}^{2}\right)\left(\left(\xi_{1}^{2}+\epsilon_{n}\right)^{\frac{\alpha_{i}}{2}}+\left(\xi_{2}^{2}+\epsilon_{n}\right)^{\frac{\beta_{i}}{2}}\right) \\
& >\beta_{i}\left(\epsilon_{n}+\left(\beta_{i}-1\right) \xi_{2}^{2}\right) \\
& \geq\left(1-\gamma_{i}\right) \beta_{i}^{2} \xi_{2}^{2}
\end{aligned}
$$

whenever $\gamma_{i} \beta_{i}>1$.

Now we look to (1.11) and (1.12). Starting with (1.11), one can follow what was done with (1.10) to require that

$$
\xi_{1}^{2}\left(\gamma_{i} \alpha_{i}-1\right) \geq-\epsilon_{n},
$$

leaving $\gamma_{i} \alpha_{i}>1$ as a sufficient condition for (1.14) to be true.

Moving on to (1.12), we note that this is equivalent to verifying when

$$
\begin{aligned}
& \left(\gamma_{i} h_{n}^{\prime \prime}\left(\xi_{1}\right)\left(h_{n}\left(\xi_{1}\right)+g_{n}\left(\xi_{2}\right)\right)+\gamma_{i}\left(\gamma_{i}-1\right) h_{n}^{\prime 2}\left(\xi_{1}\right)\right)\left(\gamma_{i} g_{n}^{\prime \prime}\left(\xi_{2}\right)\left(h_{n}\left(\xi_{1}\right)+g_{n}\left(\xi_{2}\right)\right)\right. \\
& \left.+\gamma_{i}\left(\gamma_{i}-1\right) g_{n}^{\prime 2}\left(\xi_{2}\right)\right)-\gamma_{i}^{2}\left(\gamma_{i}-1\right)^{2}{h_{n}^{\prime}}^{2}\left(\xi_{1}\right) g_{n}^{\prime 2}\left(\xi_{2}\right) \geq 0
\end{aligned}
$$

is true. Through expanding the brackets and observing some cancellations we then have

$$
\begin{aligned}
& h_{n}^{\prime \prime}\left(\xi_{1}\right) g_{n}^{\prime \prime}\left(\xi_{2}\right)\left(h_{n}\left(\xi_{1}\right)+g_{n}\left(\xi_{2}\right)\right)^{2}+\left(\gamma_{i}-1\right) h_{n}^{\prime 2}\left(\xi_{1}\right) g_{n}^{\prime \prime}\left(\xi_{2}\right)\left(h_{n}\left(\xi_{1}\right)+g_{n}\left(\xi_{2}\right)\right) \\
& +\left(\gamma_{i}-1\right) g_{n}^{\prime 2}\left(\xi_{2}\right) h_{n}^{\prime \prime}\left(\xi_{1}\right)\left(h_{n}\left(\xi_{1}\right)+g_{n}\left(\xi_{2}\right)\right) \geq 0
\end{aligned}
$$

Dividing through by $\left(h_{n}\left(\xi_{1}\right)+g_{n}\left(\xi_{2}\right)\right)>0$ we get

$$
h_{n}^{\prime \prime}\left(\xi_{1}\right) g_{n}^{\prime \prime}\left(\xi_{2}\right)\left(h_{n}\left(\xi_{1}\right)+g_{n}\left(\xi_{2}\right)\right) \geq\left(1-\gamma_{i}\right)\left(h_{n}^{\prime 2}\left(\xi_{1}\right) g_{n}^{\prime \prime}\left(\xi_{2}\right)+g_{n}^{\prime 2}\left(\xi_{2}\right) h_{n}^{\prime \prime}\left(\xi_{1}\right)\right) \text {. }
$$

Recollecting the derivatives from (1.6), (1.7), (1.8) and (1.9), the above inequality also appears as

$$
\frac{\alpha_{i}\left(\epsilon_{n}+\left(\alpha_{i}-1\right) \xi_{1}^{2}\right)\left(\xi_{1}^{2}+\epsilon_{n}\right)^{\frac{\alpha_{i}}{2}}}{\left(\xi_{1}^{2}+\epsilon_{n}\right)^{2}} \frac{\beta_{i}\left(\epsilon_{n}+\left(\beta_{i}-1\right) \xi_{2}^{2}\right)\left(\xi_{2}^{2}+\epsilon_{n}\right)^{\frac{\beta_{i}}{2}}}{\left(\xi_{2}^{2}+\epsilon_{n}\right)^{2}}
$$




$$
\begin{aligned}
& \times\left(\left(\xi_{1}^{2}+\epsilon_{n}\right)^{\frac{\alpha_{i}}{2}}+\left(\xi_{2}^{2}+\epsilon_{n}\right)^{\frac{\beta_{i}}{2}}\right) \\
& \geq\left(1-\gamma_{i}\right)\left(\frac{\alpha_{i}^{2} \xi_{1}^{2}\left(\xi_{1}^{2}+\epsilon_{n}\right)^{\alpha_{i}}}{\left(\xi_{1}^{2}+\epsilon_{n}\right)^{2}} \frac{\beta_{i}\left(\epsilon_{n}+\left(\beta_{i}-1\right) \xi_{2}^{2}\right)\left(\xi_{2}^{2}+\epsilon_{n}\right)^{\frac{\beta_{i}}{2}}}{\left(\xi_{2}^{2}+\epsilon_{n}\right)^{2}}\right. \\
& \left.+\frac{\alpha_{i}\left(\epsilon_{n}+\left(\alpha_{i}-1\right) \xi_{1}^{2}\right)\left(\xi_{1}^{2}+\epsilon_{n}\right)^{\frac{\alpha_{i}}{2}}}{\left(\xi_{1}^{2}+\epsilon_{n}\right)^{2}} \frac{\beta_{i}^{2} \xi_{2}^{2}\left(\xi_{2}^{2}+\epsilon_{n}\right)^{\beta_{i}}}{\left(\xi_{2}^{2}+\epsilon_{n}\right)^{2}}\right) .
\end{aligned}
$$

Setting $A=\left(\xi_{1}^{2}+\epsilon_{n}\right)$ and $B=\left(\xi_{2}^{2}+\epsilon_{n}\right)$, multiplying through by $A^{2} B^{2}$ gives

$$
\begin{aligned}
& \alpha_{i}\left(\epsilon_{n}+\left(\alpha_{i}-1\right) \xi_{1}^{2}\right) A^{\alpha_{i}} \beta_{i}\left(\epsilon_{n}+\left(\beta_{i}-1\right) \xi_{2}^{2}\right) B^{\frac{\beta_{i}}{2}} \\
& +\alpha_{i}\left(\epsilon_{n}+\left(\alpha_{i}-1\right) \xi_{1}^{2}\right) A^{\frac{\alpha_{i}}{2}} \beta_{i}\left(\epsilon_{n}+\left(\beta_{i}-1\right) \xi_{2}^{2}\right) B^{\beta_{i}} \\
& \geq\left(1-\gamma_{i}\right) \alpha_{i}^{2} \xi_{1}^{2} A^{\alpha_{i}} \beta_{i}\left(\epsilon_{n}+\left(\beta_{i}-1\right) \xi_{2}^{2}\right) B^{\frac{\beta_{i}}{2}} \\
& +\left(1-\gamma_{i}\right) \beta_{i}^{2} \xi_{2}^{2} B^{\beta_{i}} \alpha_{i}\left(\epsilon_{n}+\left(\alpha_{i}-1\right) \xi_{1}^{2}\right) A^{\frac{\alpha_{i}}{2}}
\end{aligned}
$$

The above inequality would hold if the following are true simultaneously:

$$
\begin{aligned}
& \alpha_{i}\left(\epsilon_{n}+\left(\alpha_{i}-1\right) \xi_{1}^{2}\right) A^{\alpha_{i}} \beta_{i}\left(\epsilon_{n}+\left(\beta_{i}-1\right) \xi_{2}^{2}\right) B^{\frac{\beta_{i}}{2}} \\
& \geq\left(1-\gamma_{i}\right) \alpha_{i}^{2} \xi_{1}^{2} A^{\alpha_{i}} \beta_{i}\left(\epsilon_{n}+\left(\beta_{i}-1\right) \xi_{2}^{2}\right) B^{\frac{\beta_{i}}{2}}
\end{aligned}
$$

and

$$
\begin{aligned}
& \alpha_{i}\left(\epsilon_{n}+\left(\alpha_{i}-1\right) \xi_{1}^{2}\right) A^{\frac{\alpha_{i}}{2}} \beta_{i}\left(\epsilon_{n}+\left(\beta_{i}-1\right) \xi_{2}^{2}\right) B^{\beta_{i}} \\
& \geq\left(1-\gamma_{i}\right) \beta_{i}^{2} \xi_{2}^{2} B^{\beta_{i}} \alpha_{i}\left(\epsilon_{n}+\left(\alpha_{i}-1\right) \xi_{1}^{2}\right) A^{\frac{\alpha_{i}}{2}} .
\end{aligned}
$$

Looking at (1.16) one can reduce the inequality to be

$$
\epsilon_{n}+\left(\alpha_{i}-1\right) \xi_{1}^{2} \geq\left(1-\gamma_{i}\right) \alpha_{i} \xi_{1}^{2}
$$

or

$$
\xi_{1}^{2}\left(\gamma_{i} \alpha_{i}-1\right) \geq-\epsilon_{n}
$$

which holds for $\gamma_{i} \alpha_{i}>1$. Similarly for (1.17) one can reduce the inequality to be

$$
\xi_{2}^{2}\left(\gamma_{i} \beta_{i}-1\right) \geq-\epsilon_{n}
$$

giving $\gamma_{i} \beta_{i}>1$ as a further sufficient condition. It is good to note that one can show that both (1.11) and (1.12) hold with a strict inequality following an analogous method. By [62, page 97] this gives $H_{\phi_{n}}$ to be positive definite. Hence, if $\alpha_{i} \gamma_{i}>1$ and $\beta_{i} \gamma_{i}>1$ the Hessian matrix of $\phi_{n}$ is both positive definite and semi-definite. 
Using now Theorem 1.3 we now have the convexity of $\phi_{n}$. This implies that the following limit must be convex:

$$
\begin{aligned}
& \lim _{n \rightarrow \infty}\left(\left(\xi_{1}^{2}+\epsilon_{n}\right)^{\frac{\alpha_{i}}{2}}+\left(\xi_{2}^{2}+\epsilon_{n}\right)^{\frac{\beta_{i}}{2}}\right)^{\gamma_{i}} \\
& =\left(\left|\xi_{1}\right|^{\alpha_{i}}+\left|\xi_{2}\right|^{\beta_{i}}\right)^{\gamma_{i}} \\
& =\psi_{i} .
\end{aligned}
$$

Hence assuming that $\alpha_{i} \gamma_{i}>1$ and $\beta_{i} \gamma_{i}>1$ we have the convexity of $\psi_{E R}$.

We will want to look at the Legendre transform of a proper convex function:

Definition 1.9. Consider a proper convex function $F: \mathbb{R}^{n} \longrightarrow \mathbb{R} \cup\{+\infty\}$. We define the Legendre transform $F^{*}: \mathbb{R}^{n} \longrightarrow \mathbb{R} \cup\{+\infty\}$ of $F$ as

$$
F^{*}\left(x^{*}\right):=\sup _{x \in \mathbb{R}^{n}}\left\{x \cdot x^{*}-F(x)\right\}
$$

Remark 1.10. As seen at [27, Chapter 14.2], the Legendre transform may not always be calculated with respect to all of the variables of a given function. To distinguish this case from when all of the variables are considered, we refer to the calculation as the partial Legendre transform. Otherwise we call it finding the (full) Legendre transform.

Proposition 1.11. If the function $F$ is proper then its Legendre transform $F^{*}$ is convex l.s.c. Additionally, if $F$ is l.s.c then

$$
\left(F^{*}\right)^{*}=F
$$

Proof. The proof can be found at [19, page 82].

The coercivity of a convex function can tell us more about its Legendre transform:

Definition 1.12. (See [18, page 216])

We call a proper convex function $F: \mathbb{R}^{n} \longrightarrow \mathbb{R} \cup\{+\infty\}$ coercive at infinity (coercive) if

$$
\lim _{\|x\| \rightarrow \infty} \frac{F(x)}{\|x\|}=\infty
$$

Proposition 1.13. The coercivity of $F$ implies that $F^{*}$ is proper in $\mathbb{R}^{n}$. If $F$ is proper then $F^{*}$ is coercive at infinity.

Proof. The proof can be found at [18, page 216]. 


\section{Example 1.14.}

A. The function $\psi_{\alpha}$ from Example 1.8.A is coercive.

B. The function $\psi_{E R}$ from Example 1.8.B is coercive whenever either $\alpha_{1} \gamma_{1}=\beta_{2} \gamma_{2}$ and $\alpha_{1} \gamma_{1}>1$, or $\beta_{1} \gamma_{1}=\alpha_{2} \gamma_{2}$ and $\beta_{1} \gamma_{1}>1$ hold. As with its convexity this is a non-trivial statement.

The proof for the coercivity of $\psi_{E R}$ requires some further estimates:

Proposition 1.15. The function $\psi_{E R}$ satisfies the following inequalities under the conditions $\alpha_{1} \gamma_{1}=\beta_{2} \gamma_{2}$ and $\alpha_{2} \gamma_{2}=\beta_{1} \gamma_{1}$ such that $\alpha_{i} \gamma_{i}>1, i=1,2$ :

A.

$$
\psi_{E R}\left(\xi_{1}, \xi_{2}\right) \geq k_{1}\left(\left|\xi_{1}\right|^{2}+\left|\xi_{2}\right|^{2}\right)^{\frac{\alpha_{1} \gamma_{1}}{2}}
$$

and

$$
\psi_{E R}\left(\xi_{1}, \xi_{2}\right) \geq k_{2}\left(\left|\xi_{1}\right|^{2}+\left|\xi_{2}\right|^{2}\right)^{\frac{\beta_{1} \gamma_{1}}{2}}
$$

for some constants $k_{1}, k_{2}>0$;

$B$.

$$
\psi_{E R}\left(\xi_{1}, \xi_{2}\right) \geq \kappa_{1}\left(\left(\left|\xi_{1}\right|^{2}+\left|\xi_{2}\right|^{2}\right)^{\frac{\alpha_{1} \gamma_{1}}{2}}+\left(\left|\xi_{1}\right|^{2}+\left|\xi_{2}\right|^{2}\right)^{\frac{\alpha_{2} \gamma_{2}}{2}}\right)
$$

and

$$
\psi_{E R}\left(\xi_{1}, \xi_{2}\right) \leq \kappa_{2}\left(\left(\left|\xi_{1}\right|^{2}+\left|\xi_{2}\right|^{2}\right)^{\frac{\alpha_{1} \gamma_{1}}{2}}+\left(\left|\xi_{1}\right|^{2}+\left|\xi_{2}\right|^{2}\right)^{\frac{\alpha_{2} \gamma_{2}}{2}}\right)
$$

for some constants $\kappa_{1}, \kappa_{2}>0$.

Proof.

A. We will prove the inequality at (1.20) taking $\alpha_{1} \gamma_{1}=\beta_{2} \gamma_{2}$ to be true. Inequality (1.21) can then be proved analogously using $\beta_{1} \gamma_{1}=\alpha_{2} \gamma_{2}$.

We first note that $\left|\xi_{1}\right|^{\alpha_{2}},\left|\xi_{2}\right|^{\beta_{1}} \geq 0$. With $\alpha_{1} \gamma_{1}=\beta_{2} \gamma_{2}$ we can then write

$$
\begin{aligned}
\psi_{E R}\left(\xi_{1}, \xi_{2}\right) & =\left(\left|\xi_{1}\right|^{\alpha_{1}}+\left|\xi_{2}\right|^{\beta_{1}}\right)^{\gamma_{1}}+\left(\left|\xi_{1}\right|^{\alpha_{2}}+\left|\xi_{2}\right|^{\beta_{2}}\right)^{\gamma_{2}} \\
& \geq\left|\xi_{1}\right|^{\alpha_{1} \gamma_{1}}+\left|\xi_{2}\right|^{\alpha_{1} \gamma_{1}}=\left\|\left(\left|\xi_{1}\right|,\left|\xi_{2}\right|\right)\right\|_{\alpha_{1} \gamma_{1}}^{\alpha_{1} \gamma_{1}} .
\end{aligned}
$$

At [47, page 9] one can find that for all $x \in \mathbb{R}^{n}$ and $p, q \geq 1$, there exists constants $c_{p, q}>0$ and $C_{p, q}>0$ such that

$$
c_{p, q}\|x\|_{q} \leq\|x\|_{p} \leq C_{p, q}\|x\|_{q}
$$

where $\|.\|_{p}$ and $\|\cdot\|_{q}$ denote the $p$-norm and $q$-norm in $\mathbb{R}^{n}$ respectively. Taking $q=2, p=\alpha_{1} \gamma_{1}$ and $n=2$ in (1.25) and using this with (1.24) one can find that

$$
\psi_{E R}\left(\xi_{1}, \xi_{2}\right) \geq\left\|\left(\left|\xi_{1}\right|,\left|\xi_{2}\right|\right)\right\|_{\alpha_{1} \gamma_{1}}^{\alpha_{1} \gamma_{1}} \geq k_{1}\left(\left|\xi_{1}\right|^{2}+\left|\xi_{2}\right|^{2}\right)^{\frac{\alpha_{1} \gamma_{1}}{2}}
$$

for some constant $k_{1}>0$. 
B. We prove here the upper bound at (1.23). The lower bound (1.22) can be proved analogously. We first rewrite $\psi_{E R}$ as

$$
\psi_{E R}\left(\xi_{1}, \xi_{2}\right)=\left\|\left(\left|\xi_{1}\right|^{\alpha_{1} \gamma_{1}},\left|\xi_{2}\right|^{\beta_{1} \gamma_{1}}\right)\right\|_{\frac{1}{\gamma_{1}}}+\left\|\left(\left|\xi_{1}\right|^{\alpha_{2} \gamma_{2}},\left|\xi_{2}\right|^{\beta_{2} \gamma_{2}}\right)\right\|_{\frac{1}{\gamma_{2}}}
$$

where $\frac{1}{\gamma_{i}}>1$ allows us to define $\|\cdot\|_{\frac{1}{\gamma_{i}}}$, the $\frac{1}{\gamma_{i}}$-norm on $\mathbb{R}^{2}, i=1,2$. Taking the conditions $\alpha_{1} \gamma_{1}=\beta_{2} \gamma_{2}$ and $\alpha_{2} \gamma_{2}=\beta_{1} \gamma_{1}$, and then using (1.25) with $p=\frac{1}{\gamma_{i}}$ and $q=1$, equation (1.27) becomes

$$
\begin{aligned}
\psi_{E R}\left(\xi_{1}, \xi_{2}\right) & \leq \tilde{\kappa}_{2}\left(\left|\xi_{1}\right|^{\alpha_{1} \gamma_{1}}+\left|\xi_{2}\right|^{\alpha_{2} \gamma_{2}}+\left|\xi_{1}\right|^{\alpha_{2} \gamma_{2}}+\left|\xi_{2}\right|^{\alpha_{1} \gamma_{1}}\right) \\
& =\tilde{\kappa}_{2}\left(\left\|\left(\left|\xi_{1}\right|,\left|\xi_{2}\right|\right)\right\|_{\alpha_{1} \gamma_{1}}^{\alpha_{1} \gamma_{1}}+\left\|\left(\left|\xi_{1}\right|,\left|\xi_{2}\right|\right)\right\|_{\alpha_{2} \gamma_{2}}^{\alpha_{2} \gamma_{2}}\right) .
\end{aligned}
$$

Here $\tilde{\kappa}_{2}>0$ and $\|\cdot\|_{\alpha_{i} \gamma_{i}}$ denotes the $\alpha_{i} \gamma_{i}$-norm on $\mathbb{R}^{2}, i=1,2$. Repeating this step with $p=\alpha_{i} \gamma_{i}$ and $q=2$ gives (1.23).

Proof of the coercivity of $\psi_{E R}$. By (1.20) we have

$$
\psi_{E R}\left(\xi_{1}, \xi_{2}\right) \geq k_{1}\left(\left|\xi_{1}\right|^{2}+\left|\xi_{2}\right|^{2}\right)^{\frac{\alpha_{1} \gamma_{1}}{2}}
$$

for $k_{1}>0$ where we have used that $\alpha_{1} \gamma_{1}=\beta_{2} \gamma_{2}$. Setting $r=\left(\left|\xi_{1}\right|^{2}+\left|\xi_{2}\right|^{2}\right)^{\frac{1}{2}}$ we can write using (1.28)

$$
\frac{\psi_{E R}\left(\xi_{1}, \xi_{2}\right)}{r} \geq k_{1} r^{\alpha_{1} \gamma_{1}-1}
$$

Assuming that $\alpha_{1} \gamma_{1}>1$, taking $r \rightarrow \infty$ in (1.29) gives by Definition 1.12 the coercivity of $\psi_{E R}$.

Following a similar method to this proof, one may also prove the coercivity of $\psi_{E R}$ using the estimate at (1.21). Here we assume that $\beta_{1} \gamma_{1}>1$ and $\beta_{1} \gamma_{1}=\alpha_{2} \gamma_{2}$ are true.

Finding the Legendre transform may be at times difficult to do when working directly from the definition. However the property of rotational invariance may facilitate the process.

Theorem 1.16. Let $F: \mathbb{R}^{n} \longrightarrow \mathbb{R} \cup\{+\infty\}$ be a proper convex function. Further suppose that it is rotational invariant, i.e. there exists a proper function $\tilde{F}:[0, \infty) \longrightarrow \mathbb{R} \cup\{+\infty\}$ such that $F(x)=\tilde{F}\left(\|x\|^{2}\right)$. Then $F^{*}$ is also rotational invariant given that

$$
\zeta_{F}(s):=4\left(\tilde{F}^{\prime}(s)\right)^{2} s
$$

is bijective for all $s \geq 0$. Further we may write $F^{*}$ explicitly as

$$
F^{*}\left(x^{*}\right)=2 \tilde{F}^{\prime}\left(\zeta_{F}^{-1}\left(\left\|x^{*}\right\|^{2}\right)\right) \zeta_{F}^{-1}\left(\left\|x^{*}\right\|^{2}\right)-\tilde{F}\left(\zeta_{F}^{-1}\left(\left\|x^{*}\right\|^{2}\right)\right) .
$$


Proof. Using that $F$ is rotational invariant we can write by Definition 1.9

$$
F^{*}\left(x^{*}\right):=\sup _{x \in \mathbb{R}^{n}}\left\{x \cdot x^{*}-\tilde{F}\left(\|x\|^{2}\right)\right\} .
$$

This supremum is achieved when

$$
x_{j}^{*}=2 x_{j} \tilde{F}^{\prime}\left(\|x\|^{2}\right), \quad j=1, \ldots, n .
$$

Squaring both sides of (1.31) and summing over $j$ we obtain

$$
\begin{aligned}
\left\|x^{*}\right\|^{2} & =4\left(\tilde{F}^{\prime}\left(\|x\|^{2}\right)\right)^{2}\|x\|^{2} \\
& =\zeta_{F}\left(\|x\|^{2}\right)
\end{aligned}
$$

Whenever $\zeta_{F}(s)$ is bijective for $s \geq 0$ we can find an inverse $\zeta_{F}^{-1}$ such that $\zeta_{F}^{-1}\left(\left\|x^{*}\right\|^{2}\right)=$ $\|x\|^{2}$. Taking this to be true and using (1.31) we have

$$
\begin{aligned}
\sup _{x \in \mathbb{R}^{n}}\left\{x \cdot x^{*}-\tilde{F}\left(\|x\|^{2}\right)\right\} & =\|x\|^{2}\left(2 \tilde{F}^{\prime}\left(\|x\|^{2}\right)\right)-\tilde{F}\left(\|x\|^{2}\right) \\
& =2 \tilde{F}^{\prime}\left(\zeta_{F}^{-1}\left(\left\|x^{*}\right\|^{2}\right)\right) \zeta_{F}^{-1}\left(\left\|x^{*}\right\|^{2}\right) \\
& -\tilde{F}\left(\zeta_{F}^{-1}\left(\left\|x^{*}\right\|^{2}\right)\right)
\end{aligned}
$$

implying that $F^{*}$ is rotational invariant.

Example 1.17. Consider the proper convex coercive function $\psi_{\alpha}$ from Example 1.8.A. We claim that

$$
\psi_{\alpha}^{*}\left(x^{*}\right)=\frac{1}{\alpha^{*}}\left\|x^{*}\right\|^{\alpha^{*}}
$$

where $\alpha^{*}=\frac{\alpha}{\alpha-1}$. Note $\psi_{\alpha}$ is rotational invariant since $\psi_{\alpha}(x)=\tilde{\psi}_{\alpha}\left(\|x\|^{2}\right)$ for $\tilde{\psi}_{\alpha}$ : $[0, \infty) \longrightarrow \mathbb{R}$ defined by $\tilde{\psi}_{\alpha}(s):=\frac{1}{\alpha} s^{\frac{\alpha}{2}}$. Hence we can use Theorem 1.16 to find $\psi_{\alpha}^{*}$. It follows that $\zeta_{\psi_{\alpha}}(s)=s^{\alpha-1}$ which is bijective for $s \geq 0$. Therefore $\psi_{\alpha}^{*}$ is rotational invariant and using (1.30) one would find $\psi_{\alpha}^{*}$ to be (1.32). Since $\psi_{\alpha}$ is continuous we know by Proposition 1.11 that $\left(\psi_{\alpha}^{*}\right)^{*}=\psi_{\alpha}$.

We will make use of the following properties found at [19, page 82]. Suppose $F, G: \mathbb{R}^{n} \longrightarrow \mathbb{R} \cup\{+\infty\}$ are both proper and convex. Then

$$
F \leq G \text { is equivalent to } F^{*} \geq G^{*}
$$

and

$$
(\lambda F)^{*}\left(x^{*}\right)=\lambda F^{*}\left(\frac{x^{*}}{\lambda}\right) \quad \text { for } \quad \lambda>0
$$


Example 1.18. Let us return to the function $\psi_{E R}$ from Example 1.8.B. Recall from Proposition 1.15 that when $\alpha_{1} \gamma_{1}=\beta_{2} \gamma_{2}$ and $\alpha_{2} \gamma_{2}=\beta_{1} \gamma_{1}$ we have

$$
\psi_{E R}\left(\xi_{1}, \xi_{2}\right) \geq k_{1}\left(\left|\xi_{1}\right|^{2}+\left|\xi_{2}\right|^{2}\right)^{\frac{\alpha_{1} \gamma_{1}}{2}}
$$

and

$$
\psi_{E R}\left(\xi_{1}, \xi_{2}\right) \leq \kappa_{2}\left(\left(\left|\xi_{1}\right|^{2}+\left|\xi_{2}\right|^{2}\right)^{\frac{\alpha_{1} \gamma_{1}}{2}}+\left(\left|\xi_{1}\right|^{2}+\left|\xi_{2}\right|^{2}\right)^{\frac{\alpha_{2} \gamma_{2}}{2}}\right)
$$

for constants $k_{1}, \kappa_{2}>0$.

With the above conditions we can learn more about $\psi_{E R}^{*}$ through (1.33). To do this we define for our own convenience the lower and upper bounds from (1.35) and (1.36) as $f: \mathbb{R}^{2} \longrightarrow \mathbb{R}$

$$
f\left(\xi_{1}, \xi_{2}\right):=k_{1}\left(\left|\xi_{1}\right|^{2}+\left|\xi_{2}\right|^{2}\right)^{\frac{\alpha_{1} \gamma_{1}}{2}}
$$

and $g: \mathbb{R}^{2} \longrightarrow \mathbb{R}$

$$
g\left(\xi_{1}, \xi_{2}\right):=\kappa_{2}\left(\left(\left|\xi_{1}\right|^{2}+\left|\xi_{2}\right|^{2}\right)^{\frac{\alpha_{1} \gamma_{1}}{2}}+\left(\left|\xi_{1}\right|^{2}+\left|\xi_{2}\right|^{2}\right)^{\frac{\alpha_{2} \gamma_{2}}{2}}\right)
$$

respectively. Both $f$ and $g$ are proper and convex on $\mathbb{R}^{2}$. Using (1.33) we obtain an upper and lower bound for the Legendre transform of $\psi_{E R}$ :

$$
f^{*}\left(x_{1}, x_{2}\right) \geq \psi_{E R}^{*}\left(x_{1}, x_{2}\right) \geq g^{*}\left(x_{1}, x_{2}\right)
$$

where $f^{*}, g^{*}: \mathbb{R}^{2} \longrightarrow \mathbb{R}$ are the Legendre transforms of $f$ and $g$ respectively.

Below we calculate $f^{*}$ :

Example 1.19. Define the proper convex function $\psi: \mathbb{R}^{n_{1}+n_{2}} \longrightarrow \mathbb{R} \cup\{+\infty\}$, $n_{i} \in \mathbb{N}, i=1,2$,

$$
\psi(\xi, \eta):=\left(\|\xi\|^{\alpha}+\|\eta\|^{\beta}\right)^{\gamma}
$$

for $1<\alpha, \beta<2,0<\gamma<1$. Here $\|$.$\| denotes the Euclidean norm. In fact \xi$ and $\eta$ are elements in different Euclidean spaces, i.e. $\|\xi\|$ and $\|\eta\|$ are Euclidean norms in $\mathbb{R}^{n_{1}}$ and $\mathbb{R}^{n_{2}}$ respectively.

One can observe that $f(\xi, \eta)=k_{1} \psi(\xi, \eta)$ where in $\psi$ we set $n_{1}=n_{2}=1$, $\alpha=\beta=2$ and $\gamma=\frac{\alpha_{1} \gamma_{1}}{2}$. Therefore, finding $f^{*}$ means to find $\left(k_{1} \psi\right)^{*}$. We can do this using (1.34) to have $\left(k_{1} \psi\right)^{*}\left(x_{1}, x_{2}\right)=k_{1} \psi^{*}\left(\frac{1}{k_{1}}\left(x_{1}, x_{2}\right)\right)$.

Under the conditions $\alpha=\beta$ and $\alpha \gamma>1$, the Legendre transform of (1.40) is a convex l.s.c function $\psi^{*}: \mathbb{R}^{n_{1}+n_{2}} \longrightarrow \mathbb{R} \cup\{+\infty\}$

$$
\psi^{*}(r, s)=\frac{(\alpha \gamma-1)}{(\gamma \alpha)^{(\alpha \gamma)^{*}}}\left(\|r\|^{\alpha^{*}}+\|s\|^{\alpha^{*}}\right)^{\frac{\gamma(\alpha-1)}{\gamma \alpha-1}} .
$$


Hence

$$
f^{*}\left(x_{1}, x_{2}\right)=\kappa_{\alpha_{1}, \gamma_{1}}\left\|\left(\left|x_{1}\right|,\left|x_{2}\right|\right)\right\|^{\left(\alpha_{1} \gamma_{1}\right)^{*}}
$$

where $\kappa_{\alpha_{1}, \gamma_{1}}=k_{1}^{\frac{-1}{\alpha_{1} \gamma_{1}-1}} \frac{\alpha_{1} \gamma_{1}-1}{\left(\alpha_{1} \gamma_{1}\right)^{\left(\alpha_{1} \gamma_{1}\right)^{*}}}$.

Therefore $\psi_{E R}^{*}$ must be bounded above by (1.42). Now we ask about its lower bound $g^{*}$ ? This will require us to find the Legendre transform of the sum of two functions. We can do this through the inf-convolute of two convex functions (see [19, Chapter II]):

Definition 1.20. Consider $F_{1}, F_{2}: \mathbb{R}^{n} \longrightarrow \mathbb{R} \cup\{+\infty\}$, both convex. The sum of their epigraphs is the following subset of $\mathbb{R}^{n} \times \mathbb{R}$ :

$$
\text { epi } F_{1}+\text { epi } F_{2}:=\left\{\left(x_{1}, a_{1}\right)+\left(x_{2}, a_{2}\right) \mid\left(x_{1}, a_{1}\right) \in \text { epi } F_{1},\left(x_{2}, a_{2}\right) \in \text { epi } F_{2}\right\} \text {. }
$$

Define now $\bar{F}: \mathbb{R}^{n} \longrightarrow \mathbb{R} \cup\{-\infty\} \cup\{+\infty\}$ by:

$$
\bar{F}(x):=\inf \left\{a \mid(x, a) \in \text { epi } F_{1}+\text { epi } F_{2}\right\} .
$$

If for every $x \in \mathbb{R}^{n}$ it holds that $\bar{F}(x)>-\infty$ then $\bar{F}$ is said to be the inf-convolute of $F_{1}$ and $F_{2}$. In this case we use the notation:

$$
\bar{F}=: F_{1} \square F_{2} \text {. }
$$

An alternative way of writing $F_{1} \square F_{2}$ is

$$
F_{1} \square F_{2}(x):=\inf _{x=x_{1}+x_{2}}\left\{F_{1}\left(x_{1}\right)+F_{2}\left(x_{2}\right)\right\}
$$

as seen at [19, page 80]. Therefore, when the right-hand side of (1.43) is strictly greater than $-\infty$ the inf-convolute of $F_{1}$ and $F_{2}$ is well-defined. Hence, if $F_{1}$ and $F_{2}$ are both bounded from below then this condition is immediately satisfied.

When well-defined $F_{1} \square F_{2}$ is convex. Further the epigraph of $F_{1} \square F_{2}$ is related to the epigraphs of $F_{1}$ and $F_{2}$ in the following way:

$$
\text { epi } F_{1}+\text { epi } F_{2} \subset \text { epi }\left(F_{1} \square F_{2}\right) \subset \overline{\text { epi } F_{1}+\text { epi } F_{2}} \text {. }
$$

However, if $F_{1}$ and $F_{2}$ are both l.s.c, this does not necessarily imply that $F_{1} \square F_{2}$ is also l.s.c. Also, if both epi $F_{1}$ and epi $F_{2}$ were closed, this does not imply the closedness of epi $F_{1}+$ epi $F_{2}$.

Proposition 1.21. Suppose $F_{1}, F_{2}: \mathbb{R}^{n} \longrightarrow \mathbb{R} \cup\{+\infty\}$ are convex l.s.c where $F_{1} \square F_{2}$ is well-defined. Then

$$
\left(F_{1} \square F_{2}\right)^{*}\left(x^{*}\right)=F_{1}^{*}+F_{2}^{*}
$$


Proof. See [19, pages 82 to 83].

Corollary 1.22. Suppose $F_{1}, F_{2}: \mathbb{R}^{n} \longrightarrow \mathbb{R} \cup\{+\infty\}$ are convex l.s.c such that $F_{1}+F_{2}$ is proper. Then $F_{1}^{*} \square F_{2}^{*}$ is well-defined and satisfies

$$
\left(F_{1}+F_{2}\right)^{*}=\left(F_{1}^{*} \square F_{2}^{*}\right)^{* *} \text {. }
$$

Proof. See [19, page 83].

The ideas of this corollary are taken further with this next theorem:

Theorem 1.23. Suppose $F_{1}, F_{2}: \mathbb{R}^{n} \longrightarrow \mathbb{R} \cup\{+\infty\}$ are convex l.s.c such that

$$
0 \in \operatorname{int}\left(\operatorname{dom} F_{1}-\operatorname{dom} F_{2}\right) \text {. }
$$

Then $F_{1}^{*} \square F_{2}^{*}$ is convex on $\mathbb{R}^{n}$ and l.s.c on $\mathbb{R}^{n}$. Further it holds that

$$
F_{1}^{*} \square F_{2}^{*}=\left(F_{1}+F_{2}\right)^{*} .
$$

Lastly, we can find some $x_{1}^{*}$ and $x_{2}^{*}$ for every $x^{*} \in \mathbb{R}^{n}$ such that

$$
\left(F_{1}^{*} \square F_{2}^{*}\right)\left(x^{*}\right)=F_{1}^{*}\left(x_{1}^{*}\right)+F_{2}^{*}\left(x_{2}^{*}\right)
$$

where $x_{1}^{*}+x_{2}^{*}=x^{*}$.

Proof. See [19, pages 87 to 89].

Now we know more about the Legendre transform of $g$ :

Example 1.24. Consider the function $g$ from (1.38). To find the Legendre transform of $g$ we choose to read it as the sum of two further functions $g_{i}: \mathbb{R}^{2} \longrightarrow \mathbb{R}$

$$
g_{i}\left(\xi_{1}, \xi_{2}\right):=\kappa_{2}\left(\left|\xi_{1}\right|^{2}+\left|\xi_{2}\right|^{2}\right)^{\frac{\alpha_{i} \gamma_{i}}{2}}, \quad i=1,2
$$

The constants $\alpha_{i}$ and $\gamma_{i}$ follow as at (1.38). We note that each $g_{i}$ is convex and continuous on $\mathbb{R}^{2}$ (hence l.s.c on $\mathbb{R}^{2}$ ). It holds that $\operatorname{dom} g_{i}=\mathbb{R}^{2}$ implying that

$$
0 \in \operatorname{int}\left(\operatorname{dom} g_{1}-\operatorname{dom} g_{2}\right)=\operatorname{int}\left(\mathbb{R}^{2}\right) .
$$

Therefore, by Theorem 1.23 we know that

$$
g_{1}^{*} \square g_{2}^{*}=\left(g_{1}+g_{2}\right)^{*} .
$$

The function $g_{i}$ is equal to $\kappa_{2} \psi$, where we have adopted $\psi$ from (1.40) and taken $n_{1}=n_{2}=1, \alpha=\beta=2$ and $\gamma=\frac{\alpha_{i} \gamma_{i}}{2}$. Hence, finding $g_{i}^{*}$ is similar to finding $f^{*}$ as was done in Example 1.19. This gives

$$
g_{i}^{*}\left(x_{1}, x_{2}\right)=\kappa_{\alpha_{i}, \gamma_{i}}\left\|\left(\left|x_{1}\right|,\left|x_{2}\right|\right)\right\|^{\left(\alpha_{i} \gamma_{i}\right)^{*}}
$$




$$
\begin{aligned}
& \text { where } \kappa_{\alpha_{i}, \gamma_{i}}=\kappa_{2}^{\frac{-1}{\alpha_{i} \gamma_{i}-1}} \frac{\alpha_{i} \gamma_{i}-1}{\left(\alpha_{i} \gamma_{i}\right)^{\left(\alpha_{i} \gamma_{i}\right)^{*}}} \text {. Using (1.46) with (1.45) we can write } \\
& g^{*}\left(x_{1}, x_{2}\right)=\inf _{\left(x_{1}, x_{2}\right)=\left(x_{1,1}, x_{1,2}\right)+\left(x_{2,1}, x_{2,2}\right)}\left\{\sum_{i=1}^{2} \kappa_{\alpha_{i}, \gamma_{i}}\left\|\left(\left|x_{i, 1}\right|,\left|x_{i, 2}\right|\right)\right\|^{\left(\alpha_{i} \gamma_{i}\right)^{*}}\right\} .
\end{aligned}
$$

Therefore the Legendre transform of $\psi_{E R}$ is bounded below by (1.47). 


\section{Chapter 2}

\section{Continuous Negative Definite Functions}

Presented here is what we need about (continuous) negative definite functions. We follow mainly [4] and [43] unless otherwise stated. When discussing Bernstein functions [60] was most helpful.

Definition 2.1. We call a function $\psi: \mathbb{R}^{n} \longrightarrow \mathbb{C}$ negative definite if for all $k \in \mathbb{N}$ and $\xi_{1}, \ldots, \xi_{k} \in \mathbb{R}^{n}$, the matrix

$$
\left(\psi\left(\xi_{l}\right)+\overline{\psi\left(\xi_{m}\right)}-\psi\left(\xi_{l}-\xi_{m}\right)\right)_{l, m=1, \ldots, k}
$$

is positive Hermitian, i.e. for any $c_{1}, \ldots, c_{k} \in \mathbb{C}$

$$
\sum_{l, m=1}^{k}\left(\psi\left(\xi_{l}\right)+\overline{\psi\left(\xi_{m}\right)}-\psi\left(\xi_{l}-\xi_{m}\right)\right) c_{l} \bar{c}_{m} \geq 0
$$

We will denote the set of all negative definite functions and continuous negative definite functions on $\mathbb{R}^{n}$ by $N\left(\mathbb{R}^{n}\right)$ and $C N\left(\mathbb{R}^{n}\right)$ respectively.

If $\psi \in N\left(\mathbb{R}^{n}\right)$ then $\psi(0) \geq 0$. Additionally, since the matrix

$$
\left(\begin{array}{cc}
\psi(\xi)+\overline{\psi(\xi)}-\psi(0) & \psi(\xi)+\overline{\psi(0)}-\psi(\xi) \\
\psi(0)+\overline{\psi(\xi)}-\psi(-\xi) & \psi(0)+\overline{\psi(0)}-\psi(0)
\end{array}\right)
$$

is positive Hermitian for every $\xi \in \mathbb{R}^{n}$, it holds that

$$
\psi(-\xi)=\overline{\psi(\xi)}
$$

The determinant of (2.1) must be non-negative which gives

$$
\operatorname{Re} \psi(\xi) \geq \psi(0) \geq 0
$$


for all $\xi \in \mathbb{R}^{n}$.

The following proposition includes further properties of negative definite functions.

\section{Proposition 2.2.}

(i) The set $N\left(\mathbb{R}^{n}\right)$ is a convex cone. It is closed under pointwise convergence.

(ii) If $\psi \in N\left(\mathbb{R}^{n}\right)$ then $\bar{\psi} \in N\left(\mathbb{R}^{n}\right)$ and $\operatorname{Re} \psi \in N\left(\mathbb{R}^{n}\right)$.

(iii) The set $N\left(\mathbb{R}^{n}\right)$ contains the non-negative constants.

(iv) The set $C N\left(\mathbb{R}^{n}\right)$ is a convex cone. It is closed with respect to uniform convergence on compact sets.

(v) For $\psi_{i} \in N\left(\mathbb{R}^{n_{i}}\right)$, $i=1,2$, the function $\psi(\xi, \eta):=\psi_{1}(\xi)+\psi_{2}(\eta)$ is a negative definite function on $\mathbb{R}^{n_{1}+n_{2}}$.

Next comes a proposition giving a way to determine more negative definite functions. The corresponding proof can be found at [4, page 40 to 41].

Proposition 2.3. Suppose $\psi: \mathbb{R}^{n} \longrightarrow \mathbb{C}$ is a negative definite function. Then $\xi \mapsto \psi(\xi)-\psi(0)$ is negative definite.

\section{Example 2.4.}

A. The function $\xi \mapsto\|\xi\|^{2 s}, 0<s \leq 1$, is a continuous negative definite function on $\mathbb{R}^{n}$.

B. For $m \geq 0$, the function $\xi \mapsto\left(\|\xi\|^{2}+m^{2}\right)^{\frac{1}{2}}-m$ is a continuous negative definite function on $\mathbb{R}^{n}$.

C. A further continuous negative definite function is given by any non-negative symmetric quadratic form $q: \mathbb{R}^{n} \times \mathbb{R}^{n} \longrightarrow \mathbb{R}$.

D. Define $\psi(\xi):=i l(\xi)$ for $l: \mathbb{R}^{n} \longrightarrow \mathbb{R}$ a linear functional. Then $\psi$ is a continuous negative definite function.

E. The function $\psi_{E R}$ from Example 1.8.B is continuous and negative definite. This is proved at Example 2.22.B.

By this next lemma we see that negative definite functions have at most quadratic growth: 
Lemma 2.5. Let $\psi \in N\left(\mathbb{R}^{n}\right)$ be locally bounded. Then there exists a constant $c_{\psi}>0$ such that

$$
|\psi(\xi)| \leq c_{\psi}\left(1+\|\xi\|^{2}\right)
$$

for all $\xi \in \mathbb{R}^{n}$.

Proof. See [43, page 134].

Lemma 2.5 also holds for any continuous negative definite function.

The following property will also be useful to us:

Proposition 2.6. Let $\psi \in N\left(\mathbb{R}^{n}\right)$ be real-valued. Then we know from equation (2.3) that $\psi(\xi) \geq 0$ for all $\xi \in \mathbb{R}^{n}$. Through this the function $\sqrt{\psi}$ is subadditive, i.e.

$$
\sqrt{\psi(\xi+\eta)} \leq \sqrt{\psi(\xi)}+\sqrt{\psi(\eta)} \text { for all } \xi, \eta \in \mathbb{R}^{n} .
$$

Proof. See [4, pages 45 to 46].

For $\psi \in N\left(\mathbb{R}^{n}\right)$ being complex-valued, the inequality at (2.4) would need $|\psi()$. for each occurrence of $\psi($.$) for it to be well-defined.$

As observed at [48] a real-valued negative definite function $\psi$ may induce a metric on $\mathbb{R}^{n}$. For such a negative definite function it holds by $(2.2)$ that $\psi(-\xi)=\psi(\xi)$, i.e. $\psi$ is even. Hence by Proposition 2.6 it must hold that every locally bounded, non-periodic negative definite function such that $\psi(0)=0$ induces a metric on $\mathbb{R}^{n}$. This is defined as $d_{\psi}: \mathbb{R}^{n} \times \mathbb{R}^{n} \longrightarrow[0, \infty)$

$$
d_{\psi}(\xi, \eta):=\sqrt{\psi(\xi-\eta)} .
$$

In fact $d_{\psi}$ is invariant under translations, i.e.

$$
d_{\psi}(\xi+\zeta, \eta+\zeta)=d_{\psi}(\xi, \eta)
$$

for all $\xi, \eta, \zeta \in \mathbb{R}^{n}$.

We ask $\psi$ to be non-periodic here since by the Lévy-Khinchine formula which we will see later at Corollary 2.16, the periodicity of $\psi$ may give multiple zeros for the function. This would condradict $\psi(0)=0$.

It will be useful later to consider balls induced by $d_{\psi}$ in the metric space $\left(\mathbb{R}^{n}, d_{\psi}\right)$. Below the sets

$$
B_{r}^{d_{\psi}}(\xi):=\left\{\eta \in \mathbb{R}^{n} \mid d_{\psi}(\xi, \eta)<r\right\}=\left\{\eta \in \mathbb{R}^{n} \mid \psi(\xi-\eta)<r^{2}\right\},
$$

and

$$
K_{r}^{d_{\psi}}(\xi):=\left\{\eta \in \mathbb{R}^{n} \mid d_{\psi}(\xi, \eta) \leq r\right\}=\left\{\eta \in \mathbb{R}^{n} \mid \psi(\xi-\eta) \leq r^{2}\right\}
$$

denote the open and closed balls respectively with radius $r>0$ and centre $\xi$.

The metric $d_{\psi}$ may generate the Euclidean topology on $\mathbb{R}^{n}$. To see this we have the lemma below: 
Lemma 2.7. Consider $\psi \in C N\left(\mathbb{R}^{n}\right)$ and the closed ball $K_{r}^{d_{\psi}}(0)$ induced by $d_{\psi}$ with radius $r>0$ and centre $0 \in \mathbb{R}^{n}$. Then $K_{r}^{d_{\psi}}(0)$ is bounded in the Euclidean topology if and only if $r^{2}<\liminf _{\|\xi\| \rightarrow \infty} \psi(\xi)$. Furthermore, $d_{\psi}$ generates on $\mathbb{R}^{n}$ the Euclidean topology if and only if $\liminf _{\|\xi\| \rightarrow \infty} \psi(\xi)>0$.

Proof. See [48, page 1106].

Following [48], whenever $d_{\psi}$ generates on $\mathbb{R}^{n}$ the Euclidean topology we call $\psi$ metric generating. Having now introduced metrics we can discuss metric measure spaces. One can find more about such spaces at [48] and [32, Chapter 1], in particular the following two definitions:

Definition 2.8. Let $(X, d), X \neq \emptyset$, be a metric space and $\mu$ a measure defined on the Borel sets of $X$. Then a triple $(X, d, \mu)$ is called a metric measure space.

Definition 2.9. Suppose $(X, d, \mu)$ is a metric measure space. Then $\mu$ is said to be doubling if

$$
\mu\left(B_{2 r}^{d}(x)\right) \leq C(\mu)\left(B_{r}^{d}(x)\right)
$$

is true for all metric balls $B_{r}^{d}(x)=\{y \in X \mid d(y, x)<r\} \subset X$ and a constant $C \geq 1$.

Example 2.10. Consider the metric measure space $\left(\mathbb{R}^{2}, d_{\psi_{E R}}, \lambda^{(2)}\right)$ where $\psi_{E R}$ is the continuous negative definite function from Example 1.8.B and $\lambda^{(2)}$ is the twodimensional Lebesgue measure. Then $\lambda^{(2)}$ is doubling whenever $\alpha_{1} \gamma_{1}=\beta_{2} \gamma_{2}, \beta_{1} \gamma_{1}=$ $\alpha_{2} \gamma_{2}, \alpha_{1} \gamma_{1}>1$ and $\beta_{1} \gamma_{1}>1$. This is another non-trivial statement and we provide the proof below.

Proof that $\lambda^{(2)}$ is doubling. We begin by noting that the Lebesgue measure is translation invariant. Hence following (2.5) it will be sufficient to prove that

$$
\lambda^{(2)}\left(B_{2 r}^{d_{\psi_{E R}}}(0)\right) \leq C \lambda^{(2)}\left(B_{r}^{d_{\psi_{E R}}}(0)\right)
$$

where $C \geq 1$. We proved in Proposition 1.15 the following estimates involving $\psi_{E R}$ :

$$
\psi_{E R}\left(\xi_{1}, \xi_{2}\right) \geq \kappa_{1}\left(\left(\left|\xi_{1}\right|^{2}+\left|\xi_{2}\right|^{2}\right)^{\frac{\alpha_{1} \gamma_{1}}{2}}+\left(\left|\xi_{1}\right|^{2}+\left|\xi_{2}\right|^{2}\right)^{\frac{\alpha_{2} \gamma_{2}}{2}}\right)
$$

and

$$
\psi_{E R}\left(\xi_{1}, \xi_{2}\right) \leq \kappa_{2}\left(\left(\left|\xi_{1}\right|^{2}+\left|\xi_{2}\right|^{2}\right)^{\frac{\alpha_{1} \gamma_{1}}{2}}+\left(\left|\xi_{1}\right|^{2}+\left|\xi_{2}\right|^{2}\right)^{\frac{\alpha_{2} \gamma_{2}}{2}}\right)
$$

for some constants $\kappa_{1}, \kappa_{2}>0$ and $\alpha_{1} \gamma_{1}=\beta_{2} \gamma_{2}, \beta_{1} \gamma_{1}=\alpha_{2} \gamma_{2}$. For $a, b \geq 0$ we have

$$
\frac{1}{\sqrt{2}}(a+b) \leq\left(a^{2}+b^{2}\right)^{\frac{1}{2}} \leq a+b,
$$


hence

$$
\frac{1}{\sqrt{2}} \max (a, b) \leq\left(a^{2}+b^{2}\right)^{\frac{1}{2}} \leq 2 \max (a, b) .
$$

Let $a^{2}=\|\xi\|^{\alpha_{1} \gamma_{1}}$ and $b^{2}=\|\xi\|^{\alpha_{2} \gamma_{2}}$ where $\|$.$\| is the Euclidean norm in \left(\xi_{1}, \xi_{2}\right)=\xi \in$ $\mathbb{R}^{2}$. Then combining the above with the estimates at (2.7) and (2.8) we have

$$
\sqrt{\frac{\kappa_{1}}{2}} \max \left(\|\xi\|^{\frac{\alpha_{1} \gamma_{1}}{2}},\|\xi\|^{\frac{\alpha_{2} \gamma_{2}}{2}}\right) \leq \psi_{E R}^{\frac{1}{2}}(\xi) \leq 2 \sqrt{\kappa_{2}} \max \left(\|\xi\|^{\frac{\alpha_{1} \gamma_{1}}{2}},\|\xi\|^{\frac{\alpha_{2} \gamma_{2}}{2}}\right) .
$$

Let us define a new metric on $\mathbb{R}^{n}, n \in \mathbb{N}$, namely $d_{\alpha}: \mathbb{R}^{n} \times \mathbb{R}^{n} \longrightarrow \mathbb{R}$

$$
d_{\alpha}(\xi, \eta):=\|\xi-\eta\|^{\frac{\alpha}{2}}
$$

for $1<\alpha<2$. Assuming $\alpha_{1} \gamma_{1}>1$ and $\beta_{1} \gamma_{1}>1$, we have $1<\alpha_{i} \gamma_{i}<2$ and $1<\beta_{i} \gamma_{i}<2$ for $i=1,2$. Taking $n=2$ at (2.10), we can then use $d_{\alpha}$ with (2.9) to have

$$
\begin{aligned}
& B_{r}^{d_{\psi_{E R}}}(0)=\left\{\xi \in \mathbb{R}^{2} \mid \psi_{E R}^{\frac{1}{2}}(\xi)<r\right\} \\
& \subset\left\{\xi \in \mathbb{R}^{2} \mid \max \left(\|\xi\|^{\frac{\alpha_{1} \gamma_{1}}{2}},\|\xi\|^{\frac{\alpha_{2} \gamma_{2}}{2}}\right)<\sqrt{\frac{2 r^{2}}{\kappa_{1}}}\right\} \\
&=\left\{\xi \in \mathbb{R}^{2} \mid\|\xi\|^{\frac{\alpha_{1} \gamma_{1}}{2}}<\sqrt{\frac{2 r^{2}}{\kappa_{1}}},\|\xi\|^{\frac{\alpha_{2} \gamma_{2}}{2}}<\sqrt{\frac{2 r^{2}}{\kappa_{1}}}\right\} \\
&=B_{\sqrt{\frac{2 r^{2}}{\kappa_{1}}}}^{d_{\alpha_{1} \gamma_{1}}}(0) \cap B^{d_{\alpha_{2} \gamma_{2}}}(0) . \\
& \sqrt{\frac{2 r^{2}}{\kappa_{1}}}
\end{aligned}
$$

Similarly we may also deduce

$$
\begin{aligned}
B_{\frac{r}{2 \sqrt{\kappa_{2}}}}^{d_{\alpha_{1} \gamma_{1}}}(0) \cap B_{\frac{r}{2 \sqrt{\kappa_{2}}}}^{d_{\alpha_{2} \gamma_{2}}}(0) & =\left\{\xi \in \mathbb{R}^{2} \mid\|\xi\|^{\frac{\alpha_{1} \gamma_{1}}{2}}<\frac{r}{2 \sqrt{\kappa_{2}}},\|\xi\|^{\frac{\alpha_{2} \gamma_{2}}{2}}<\frac{r}{2 \sqrt{\kappa_{2}}}\right\} \\
& =\left\{\xi \in \mathbb{R}^{2} \mid 2 \sqrt{\kappa_{2}} \max \left(\|\xi\|^{\frac{\alpha_{1} \gamma_{1}}{2}},\|\xi\|^{\frac{\alpha_{2} \gamma_{2}}{2}}\right)<r\right\} \\
& \subset\left\{\xi \in \mathbb{R}^{2} \mid \psi_{E R}^{\frac{1}{2}}(\xi)<r\right\} \\
& =B_{r}^{d_{\psi_{E R}}}(0) .
\end{aligned}
$$

Hence overall we have

$$
B_{\frac{r}{2 \sqrt{\kappa_{2}}}}^{d_{\alpha_{1} \gamma_{1}}}(0) \cap B_{\frac{r}{2 \sqrt{\kappa_{2}}}}^{d_{\alpha_{2} \gamma_{2}}}(0) \subset B_{r}^{d_{\psi_{E R}}}(0) \subset B_{\sqrt{\frac{2 r^{2}}{\kappa_{1}}}}^{d_{\alpha_{1} \gamma_{1}}}(0) \cap B_{\sqrt{\frac{2 r^{2}}{\kappa_{1}}}}^{d_{\alpha_{2} \gamma_{2}}}(0)
$$


Taking the two-dimensional Lebesgue measure of the that in the inclusion at (2.11) we have

$$
\begin{aligned}
\lambda^{(2)}\left(B_{\frac{r}{2 \sqrt{\kappa_{2}}}}^{d_{\alpha_{1} \gamma_{1}}}(0) \cap B_{\frac{r}{2 \sqrt{\kappa_{2}}}}^{d_{\alpha_{2} \gamma_{2}}}(0)\right) & \leq \lambda^{(2)}\left(B_{r}^{d_{\psi_{E R}}}(0)\right) \\
& \leq \lambda^{(2)}\left(\begin{array}{c}
B^{d_{\alpha_{1} \gamma_{1}}}(0) \cap B^{\frac{2 r^{2}}{\kappa_{1}}} \sqrt{\frac{2 r^{2}}{\kappa_{1}}}(0)
\end{array}\right) .
\end{aligned}
$$

To proceed we consider when $\alpha_{l} \gamma_{l} \geq \alpha_{m} \gamma_{m}$, for $l, m=1,2$ and $l \neq m$. This implies that

$$
B_{\frac{r}{2 \sqrt{\kappa_{2}}}}^{d_{\alpha_{l} \gamma_{l}}}(0) \cap B_{\frac{r}{2 \sqrt{\kappa_{2}}}}^{d_{\alpha_{m} \gamma_{m}}}(0)=B_{\frac{r}{2 \sqrt{\kappa_{2}}}}^{d_{\alpha_{l} \gamma_{l}}}(0)
$$

and

$$
B_{\sqrt{\frac{2 r^{2}}{\kappa_{1}}}}^{d_{\alpha_{l} \gamma_{l}}}(0) \cap B_{\sqrt{\frac{2 r^{2}}{\kappa_{1}}}}^{d_{\alpha_{m} \gamma_{m}}}(0)=B^{d_{\alpha_{l} \gamma_{l}}}(0) .
$$

Further, consider the metric measure space $\left(\mathbb{R}^{2}, d_{\alpha}, \lambda^{(2)}\right)$. Then by [52, pages 109 and 110] it holds that $\lambda^{(2)}$ doubling. Using this with (2.12) as well as (2.13) and (2.14) above, we have

$$
\begin{aligned}
\lambda^{(2)}\left(B_{2 r}^{d_{\psi_{E R}}}(0)\right) & \leq \lambda^{(2)}\left(\begin{array}{c}
B_{\alpha_{l} \gamma_{l}}^{d_{\sqrt{\frac{22}{\kappa_{1}}}}}(0) \\
2
\end{array}\right) \leq \kappa \lambda^{(2)}\left(\begin{array}{c}
B_{\sqrt{\frac{2 r^{2}}{\kappa_{1}}}}^{d_{\alpha_{l}}}(0) \\
\sqrt{\kappa^{2}}
\end{array}\right)^{\frac{2}{\alpha_{l} \gamma_{l}}}=\kappa \pi\left(\frac{8 \kappa_{2}}{\kappa_{1}}\right)^{\frac{2}{\alpha_{l} \gamma_{l}}}\left(\frac{r}{2 \sqrt{\kappa_{2}}}\right)^{\frac{4}{\alpha_{l} \gamma_{l}}} \\
& =\kappa \pi\left(\lambda^{(2)}\left(B_{\frac{r}{2 \kappa_{\kappa_{2}}}}^{d_{\alpha_{l} \gamma_{l}}}(0)\right)\right. \\
& \leq C \lambda^{(2)}\left(B_{r}^{d_{\psi_{E R}}}(0)\right)
\end{aligned}
$$

where we define the constant $C:=\kappa\left(\frac{8 \kappa_{2}}{\kappa_{1}}\right)^{\frac{2}{\alpha_{l} \gamma_{l}}}$. Note $\kappa>0$ was the constant obtained using that $\lambda^{(2)}$ is doubling when considered as a part of the metric measure spaces $\left(\mathbb{R}^{2}, d_{\alpha_{l} \gamma_{l}}, \lambda^{(2)}\right)$. The constants $\kappa_{1}, \kappa_{2}>0$ were chosen arbitrarily. Hence when forming the estimate (2.9), we can always choose these constants such that $\kappa_{2}>\kappa_{1}$. Therefore it always holds that $C \geq 1$. Overall we now have (2.6).

It is known that continuous negative definite functions have a one-to-one correspondence with convolution semigroups on $\mathbb{R}^{n}$. This requires us to know about positive definite functions: 
Definition 2.11. We call a function $u: \mathbb{R}^{n} \longrightarrow \mathbb{C}$ positive definite if the matrix $\left(u\left(\xi_{l}-\xi_{m}\right)\right)_{l, m=1, \ldots, k}$ is positive Hermitian for all $k \in \mathbb{N}$ and $\xi_{1}, \ldots, \xi_{k} \in \mathbb{R}^{n}$.

Definition 2.12. We call a family $\left(\mu_{t}\right)_{t \geq 0}$ of bounded Borel measures on $\mathbb{R}^{n}$ a convolution semigroup on $\mathbb{R}^{n}$ if the following conditions are satisfied:

(i) for all $t \geq 0$ it holds that $\mu_{t}\left(\mathbb{R}^{n}\right) \leq 1$;

(ii) $\mu_{s} * \mu_{t}=\mu_{t+s}$ for $s, t \geq 0$ and $\mu_{0}=\epsilon_{0}$;

(iii) $\mu_{t} \rightarrow \epsilon_{0}$ vaguely as $t \rightarrow 0$.

Here $\epsilon_{0}$ denotes the Dirac measure at $0 \in \mathbb{R}^{n}$.

Theorem 2.13. Let $\left(\mu_{t}\right)_{t \geq 0}$ be a convolution semigroup on $\mathbb{R}^{n}$. Then there exists a uniquely determined continuous negative definite function $\psi: \mathbb{R}^{n} \longrightarrow \mathbb{C}$ such that

$$
\hat{\mu}_{t}(\xi)=(2 \pi)^{-\frac{n}{2}} e^{-t \psi(\xi)} \quad \text { for } \quad t \geq 0 \quad \text { and } \quad \xi \in \mathbb{R}^{n} .
$$

Conversely, let $\psi: \mathbb{R}^{n} \longrightarrow \mathbb{C}$ be a continuous negative definite function. Then there exists a unique convolution semigroup $\left(\mu_{t}\right)_{t \geq 0}$ on $\mathbb{R}^{n}$ such that (2.15) is true.

Proof. See [43, pages 127 to 129].

Following Theorem 2.13 one can obtain:

Corollary 2.14. A function $\psi: \mathbb{R}^{n} \longrightarrow \mathbb{C}$ is continuous and negative definite if and only if

(i) $\psi(0) \geq 0$;

(ii) $\xi \mapsto e^{-t \psi(\xi)}$ is continuous and positive definite for $t>0$.

A continuous negative definite function can be characterised by what is known as the Lévy-Khinchine formula:

Theorem 2.15. Suppose $\psi: \mathbb{R}^{n} \longrightarrow \mathbb{C}$ is a continuous negative definite function. Then there exists a constant $c \geq 0$, a vector $d \in \mathbb{R}^{n}$, a positive symmetric semidefinite quadratic form $q$ on $\mathbb{R}^{n}$ and a finite symmetric measure $\mu$ on $\mathbb{R}^{n} \backslash\{0\}$ such that

$$
\psi(\xi)=c+i(d \cdot \xi)+q(\xi)+\int_{\mathbb{R}^{n} \backslash\{0\}}\left(1-e^{-i x \cdot \xi}-\frac{i x \cdot \xi}{1+\|x\|^{2}}\right) \frac{1+\|x\|^{2}}{\|x\|^{2}} \mu(\mathrm{d} x)
$$

is true. Conversely, given $c, d, q$ and $\mu$ as above, a continuous negative definite function is defined by (2.16). Furthermore, $\psi$ uniquely determines $c, d, q$ and $\mu$. 
Proof. See [43, pages 148 to 151].

The Lévy-Khinchine formula of real-valued continuous negative definite functions follows as:

Corollary 2.16. Consider $\psi: \mathbb{R}^{n} \longrightarrow \mathbb{R}$, a real-valued continuous negative definite function. Then with $c, q$ and $\mu$ as in Theorem 2.15 the function $\psi$ has the representation:

$$
\psi(\xi)=c+q(\xi)+\int_{\mathbb{R}^{n} \backslash\{0\}}(1-\cos (x \cdot \xi)) \frac{1+\|x\|^{2}}{\|x\|^{2}} \mu(\mathrm{d} x) .
$$

Definition 2.17. Suppose $\mu$ is the measure in the Lévy-Khinchine formula for $\psi \in$ $C N\left(\mathbb{R}^{n}\right)$. We call the measure

$$
\nu(\mathrm{d} x)=\frac{1+\|x\|^{2}}{\|x\|^{2}} \mu(\mathrm{d} x)
$$

the Lévy measure defined on $\mathcal{B}\left(\mathbb{R}^{n} \backslash\{0\}\right)$ associated with $\psi$.

Looking at the Lévy-Khinchine formula it is clear that a continuous negative definite function is not necessarily differentiable. This key observation will be important as we look to the mechanics associated with negative definite functions later in Chapter 3.

More continuous negative definite functions may be constructed using Bernstein functions.

Definition 2.18. We call a function $f:(0, \infty) \longrightarrow \mathbb{R}$ a Bernstein function if

(i) $f \in C^{\infty}((0, \infty))$;

(ii) for all $x>0$ it holds that $f(x) \geq 0$;

(iii) for all $k \in \mathbb{N}$ and $x>0$ it holds that $(-1)^{k-1} f^{(k)}(x) \geq 0$.

Examples of Bernstein functions are as follows:

\section{Example 2.19.}
A. $x \mapsto x^{\alpha} \quad$ for $\quad 0<\alpha<1$;
B. $x \mapsto(x+1)^{\alpha}-1 \quad$ for $0<\alpha<1$;
C. $x \mapsto \frac{x}{a+x} \quad$ for $\quad a>0$; 
D. $x \mapsto \log \left(1+\frac{x}{a}\right) \quad$ for $\quad a>0$;

E. $x \mapsto \sqrt{x} \arctan \left(\sqrt{\frac{x}{a}}\right)$ for $a>0$.

As mentioned at [60, page 142], one can show that for a Bernstein function $f$ and a continuous negative definite function $\psi, f \circ \psi$ may be given by a Lévy-Khinchine formula. By Theorem $2.15 f \circ \psi$ must also be continuous negative definite. This result is also given at [43] as follows:

Lemma 2.20. Let $f$ be any Bernstein function and $\psi$ any continuous negative definite function on $\mathbb{R}^{n}$ such that $f(0)=0$. Then $f \circ \psi$ is also continuous and negative definite.

Proof. We refer to [43, page 189].

Later we will consider continuous negative definite functions that are rotationally invariant. Hence this result due to Schoenberg and Bochner will be relevant to us:

Theorem 2.21. A function $f:(0, \infty) \longrightarrow[0, \infty)$ is a Bernstein function if and only if $\xi \mapsto f\left(\|\xi\|^{2}\right), \xi \in \mathbb{R}^{n}$, is continuous negative definite for all $n \in \mathbb{N}$.

Proof. See [60, pages 142 to 144].

\section{Example 2.22.}

A. The function $\psi_{\alpha}$ from Example 1.8.A is continuous negative definite. Recalling that it is rotatinal invariant from Example 1.17 we can write $\psi_{\alpha}(x)=\tilde{\psi}_{\alpha}\left(\|x\|^{2}\right)$ for $\tilde{\psi}_{\alpha}:[0, \infty) \longrightarrow \mathbb{R}$ defined as $\tilde{\psi}_{\alpha}(s):=\frac{1}{\alpha} s^{\frac{\alpha}{2}}$. Comparing to Theorem 2.21 we see its claims to be justified as we recognise $\tilde{\psi}_{\alpha}$ as a Bernstein function by Example 2.19.A.

B. Recall the function $\psi_{E R}$ from Example 1.8.B. We claim that $\psi_{E R}$ is an element of $C N\left(\mathbb{R}^{2}\right)$. First, using Example 2.4.A. we note that $\left|\xi_{1}\right|^{\alpha_{i}},\left|\xi_{2}\right|^{\beta_{i}} \in C N(\mathbb{R})$ for $i=1,2$. This implies by Proposition 2.2 (v) that $\left|\xi_{1}\right|^{\alpha_{i}}+\left|\xi_{2}\right|^{\beta_{i}} \in C N\left(\mathbb{R}^{2}\right)$. Hence, following Lemma 2.20 we have $\left(\left|\xi_{1}\right|^{\alpha_{i}}+\left|\xi_{2}\right|^{\beta_{i}}\right)^{\gamma_{i}} \in C N\left(\mathbb{R}^{2}\right)$ where we note that $x \mapsto x^{\gamma_{i}}$ is a Bernstein function by Example 2.19.A. Lastly, recollecting that $C N\left(\mathbb{R}^{2}\right)$ is a convex cone we can deduce that

$$
\psi_{E R}\left(\xi_{1}, \xi_{2}\right)=\left(\left|\xi_{1}\right|^{\alpha_{1}}+\left|\xi_{2}\right|^{\beta_{1}}\right)^{\gamma_{1}}+\left(\left|\xi_{1}\right|^{\alpha_{2}}+\left|\xi_{2}\right|^{\beta_{2}}\right)^{\gamma_{2}} \in C N\left(\mathbb{R}^{2}\right) .
$$




\section{Chapter 3}

\section{Hamilton and Lagrange Functions Associated with Negative Definite Functions}

We want to study the Hamiltonian dynamics associated with the function

$$
\mathcal{H}(q, p)=\psi(p)+V(q)
$$

where $\mathcal{H} \in C^{1}\left(\mathbb{R}^{2 n}\right)$ is convex with respect to $p$. We also assume $V \in C^{1}\left(\mathbb{R}^{n}\right)$ and $\psi \in C N\left(\mathbb{R}^{n}\right) \cap C^{1}\left(\mathbb{R}^{n}\right)$ is real-valued, convex and coercive. As we will see there will be cases when the Hamilton function has singularities upon differentiating. Hence $\mathcal{H}$ may not always be assumed to be differentiable on all of $\mathbb{R}^{2 n}$. In this case the domains of such Hamilton functions may be changed and this will always be made clear.

To continue with the study of the Hamiltonian dynamics of (3.1), we begin with a brief revision of classical mechanics, in particular what are mechanical systems and their associated Hamiltonians, as well as how their behavior may be determined by solving what are known as Hamilton's or Lagrange's equations. This will then provide a good reference for when we develop an analogous Hamiltonian dynamics for the function (3.1). The material will be in reference to [2] and [26] with any further references being specified as the reader progresses. Another interpretation of the Hamilton function and Hamilton's equations will be covered later where we use the calculus of variations.

\subsection{Revising Classical Mechanics}

An aim in classical mechanics is to study the motion of a system of $N$ particles in $\mathbb{R}^{3}$ during an interval of time. Such a system is an example of a mechanical system. 
To study the motion a concept of position and movement needs to be established:

Given a time $t \in I \subset \mathbb{R}$ the position vector of the $i$ th particle in $\mathbb{R}^{3}$ at $t$ is given by the mappings $\underline{r}_{i}: I \longrightarrow \mathbb{R}^{3}$

$$
\underline{r}_{i}(t)=\left(x_{i}(t), y_{i}(t), z_{i}(t)\right), \quad i=1, \ldots, N .
$$

This position is taken with respect to an origin $(0,0,0) \in \mathbb{R}^{3}$, hence (3.1.1) specifies the displacement from the origin. The image of $(3.1 .1), \underline{r}_{i}(I)$, is called a curve in $\mathbb{R}^{3}$. As time passes the position of the particle travels proportionally along the curve. Because of this $\underline{r}_{i}(t)$ is also referred to as the motion of the particle.

Whenever $x_{i}, y_{i}, z_{i} \in C^{2}(I)$ we can determine the velocity and acceleration of each particle. The velocity vector of the $i$ th particle at $t \in I$ is given by $\underline{\dot{r}}_{i}: I \longrightarrow$ $\mathbb{R}^{3}$

$$
\underline{\dot{r}}_{i}(t)=\left(\dot{x}_{i}(t), \dot{y}_{i}(t), \dot{z}_{i}(t)\right) .
$$

Similarly the acceleration vector of the $i$ th particle at $t \in I$ is given by $\ddot{\ddot{q}}_{i}: I \longrightarrow$ $\mathbb{R}^{3}$

$$
\ddot{\underline{x}}_{i}(t)=\left(\ddot{x}_{i}(t), \ddot{y}_{i}(t), \ddot{z}_{i}(t)\right) .
$$

The Cartesian product of the images $\underline{r}_{i}(I)$ forms the a $3 N$-dimensional space given by $\underline{r}: I \longrightarrow \mathbb{R}^{3 N}$

$$
\underline{r}(t)=\left(x_{1}(t), y_{1}(t), z_{1}(t), \ldots, x_{N}(t), y_{N}(t), z_{N}(t)\right) .
$$

Similar mappings for the velocity and acceleration vectors can be defined as $\underline{\dot{r}}$ : $I \longrightarrow \mathbb{R}^{3 N}$

$$
\underline{\dot{r}}(t)=\left(\dot{x}_{1}(t), \dot{y}_{1}(t), \dot{z}_{1}(t), \ldots, \dot{x}_{N}(t), \dot{y}_{N}(t), \dot{z}_{N}(t)\right),
$$

and $\ddot{\ddot{x}}: I \longrightarrow \mathbb{R}^{3 N}$

$$
\underline{\underline{r}}(t)=\left(\ddot{x}_{1}(t), \ddot{y}_{1}(t), \ddot{z}_{1}(t), \ldots, \ddot{x}_{N}(t), \ddot{y}_{N}(t), \ddot{z}_{N}(t)\right)
$$

respectively. We can think of $\underline{r}, \underline{\dot{r}}$ and $\underline{\ddot{q}}$ as describing the movement of all $N$ particles as a whole.

Given an initial time for the motion, $t_{0}$, the vector $\underline{r}(t)$ is uniquely determined by the initial values $\underline{r}\left(t_{0}\right)$ and $\underline{\dot{r}}\left(t_{0}\right)$. In fact, the acceleration $\underline{\ddot{r}}(t)$ is determined by $\underline{r}\left(t_{0}\right)$ and $\underline{\dot{r}}\left(t_{0}\right)$ through

$$
m_{i} \ddot{\ddot{x}}_{i}(t)=\underline{F}_{i}(\underline{r}(t), \underline{\dot{r}}(t), t)
$$

for a function $\underline{F}_{i}: \mathbb{R}^{3 N} \times \mathbb{R}^{3 N} \times \mathbb{R} \longrightarrow \mathbb{R}^{3 N}$. This function represents all the forces acting on the $i$ th particle. The constant $m_{i}$ denotes the mass of the $i$ th particle. As at [51, Chapter 1], equation (3.1.7) is the equation of motion for the $i$ th particle. It may be read as a second-order ordinary differential equation which can be solved as an initial value problem with $2 N$ initial conditions. 
Here we take the assumption that the corresponding system to (3.1.7) occurs within an inertial frame. This means that the system or (3.1.7) is assumed to be invariant with respect to transformations such as rotating the coordinate axes or translating the origin.

The momentum of the $i$ th particle is given by $\underline{p}_{i}: I \longrightarrow \mathbb{R}^{3}$

$$
\underline{p}_{i}(t)=\left(m_{i} \dot{x}_{i}(t), m_{i} \dot{y}_{i}(t), m_{i} \dot{z}_{i}(t)\right)
$$

with the Cartesian product of the images $\underline{p}_{i}(I)$ given by $\underline{p}: I \longrightarrow \mathbb{R}^{3 N}$

$$
\underline{p}(t)=\left(m_{1} \dot{x}_{1}(t), m_{1} \dot{y}_{1}(t), m_{1} \dot{z}_{1}(t), \ldots, m_{N} \dot{x}_{N}(t), m_{N} \dot{y}_{N}(t), m_{N} \dot{z}_{N}(t)\right) .
$$

Also of use to know is the total kinetic energy of the system given by $T:=$ $\sum_{i=1}^{N} \frac{1}{2} m_{i}\left|\underline{\underline{r}}_{i}(t)\right|^{2}$. The potential energy $V\left(\underline{r}_{i}(t)\right)$ of the $i$ th particle is a differentiable function $V_{i}: \mathbb{R}^{3} \longrightarrow \mathbb{R}$ such that

$$
\underline{F}_{i}=-\nabla V_{i}\left(\underline{r}_{i}(t)\right)=\left(-\frac{\partial V_{i}}{\partial x_{i}}(t),-\frac{\partial V_{i}}{\partial y_{i}}(t),-\frac{\partial V_{i}}{\partial z_{i}}(t)\right) .
$$

In this case the forces $\underline{F}_{i}$ are said to be conservative. The potential energy of the system is $V: \mathbb{R}^{3 N} \longrightarrow \mathbb{R}$ given by $V(\underline{r}(t))=\left(V_{1}\left(\underline{r}_{i}(t)\right), \ldots, V_{N}\left(\underline{r}_{i}(t)\right)\right)$.

In classical mechanics, it is possible to have constraints that restrict the motion of the system. There exists a way to classify them.

Definition 3.1.1. Let $\underline{r}_{i}, i=1, \ldots, N$, be the position vectors of $N$ particles in a mechanical system. Further, suppose the conditions of a constraint can be expressed as equations of the form

$$
f\left(x_{1}(t), y_{1}(t), z_{1}(t), \ldots, x_{N}(t), y_{N}(t), z_{N}(t), t\right)=0
$$

for some real-valued function $f$. Then the constraint is called holonomic.

A problem with imposing constraints is that the coordinates determining the position are no longer independent due to the existence of equations such as (3.1.11). This implies that the equations of motion of each particle contained in (3.1.7) are also not all independent. Because of this it is difficult to obtain explicit expressions when determining $\underline{r}, \underline{\dot{r}}$ and $\underline{\ddot{r}}$. When working with holonomic constraints one can overcome this problem through introducing generalised coordinates.

Given a mechanical system, suppose there are $k$ holonomic constraints given by

$$
f_{l}\left(x_{1}(t), y_{1}(t), z_{1}(t), \ldots, x_{N}(t), y_{N}(t), z_{N}(t), t\right)=0, \quad l=1, \ldots, k .
$$


The system originally has $3 N$ independent coordinates determining the position of the $N$ particles. Introducing $k$ equations of the form (3.1.12), it is possible to eliminate $k$ of these $3 N$ coordinates, leaving $3 N-k$ independent coordinates. These are the generalised coordinates. We call the space of all generalised coordinates the configuration space. For simplicity let the number $3 N-k$ be $n$ such that we have $n$ generalised coordinates $q_{j}, j=1, \ldots, n$. The number $n$ is called the number of degrees of freedom of the system. Through this the position vectors of the particles may be written as invertible functions of the generalised coordinates and time:

$$
\underline{r}_{i}(t)=\underline{r}_{i}\left(q_{1}(t), \ldots, q_{n}(t), t\right), \quad i=1, \ldots, N .
$$

Equations (3.1.13) therefore serve as transformation equations between the two sets of coordinates $\left(x_{i}(t), y_{i}(t), z_{i}(t)\right)_{i=1, \ldots, N}$ and $\left(q_{j}(t)\right)_{j=1, \ldots, n}$.

The configuration space can be thought of as an $n$-dimensional space of points $q=\left(q_{1}(t), \ldots, q_{n}(t)\right)$. As time passes, a path is traced proportianally throughout this space representing the motion of the particles. This is called the "path of motion of the system". Note that a point in the configuration space does not represent the motion of any single particle but the motion of all the particles as a whole.

Here is an example of finding the generalised coordinates for a constrained system of one particle. For simiplicity, the particle is chosen to move within $\mathbb{R}^{2}$ but all the ideas so far discussed can be applied analogously to a space of $N$ particles moving in $\mathbb{R}^{2}$.

Example 3.1.2. Consider a particle moving in $\mathbb{R}^{2}$. The position vector of the particle at $t \in I$ is given by $\underline{r}: I \longrightarrow \mathbb{R}^{2}, \underline{r}(t)=(x(t), y(t))$. Let us introduce $a$ constraint on the system. Suppose the particle may only follow the path of an ellipse such that the position of the particle at $t \in I$ satisfies

$$
\frac{x^{2}(t)}{a^{2}}+\frac{y^{2}(t)}{b^{2}}-1=0 .
$$

Note (3.1.14) is of the form of (3.1.11) and so this constraint is holonomic.

The system was initially described using two coordinates $x(t)$ and $y(t)$. We now have one equation of constraint and therefore one of the coordinates may be eliminated. Here we eliminate $x(t)$ but the discussion may be carried out analogously when eliminating $y(t)$. Rearranging (3.1.14) for $x(t)$ we have

$$
x(t)= \pm a \sqrt{1-\frac{y^{2}(t)}{b^{2}}}
$$

giving the position vector now to be

$$
\underline{r}(t)=\left( \pm a \sqrt{1-\frac{y^{2}(t)}{b^{2}}}, y(t)\right) .
$$


Therefore the system is now determined by a single generalised coordinate $y(t)$ and so we could write $\underline{r}(t)=\underline{r}(y(t), t)$ to fit the form of (3.1.13). The configuration space in this case is $\mathbb{R}$ with one degree of freedom.

As was possible with the position vectors, we can define the corresponding generalised velocity vectors through taking the time derivative of the generalised coordinates: $\dot{q}(t)=\left(\dot{q}_{1}(t), \ldots, \dot{q}_{n}(t)\right)$. As seen at [27, Chapter 12] this is calculated as seen below

$$
\underline{\dot{r}}_{i}(t)=\sum_{j=1}^{n} \frac{\partial \underline{r}_{i}}{\partial q_{j}} \dot{q}_{j}+\frac{\partial \underline{r}_{i}}{\partial t}
$$

implying that the velocity vectors are functions of $q(t)$ and $\dot{q}(t)$ :

$$
\dot{\underline{r}}_{i}(t)=\underline{\dot{r}}_{i}\left(q_{1}(t), \ldots, q_{n}(t), \dot{q}_{1}(t), \ldots, \dot{q}_{n}(t), t\right), \quad i=1, \ldots, N .
$$

A second problem with introducing constraints is that some forces contained in $\underline{F}_{i}$ may be unknown. From here an alternate representation of the equations of motion is derived to counter the problem. Here we list the main result in doing this but the full details can be found at [26] and [27].

When a system is determined by positions (3.1.13), the corresponding kinetic energy is of a quadratic form denoted $T(q(t), \dot{q}(t), t)$. Introducing generalised coordinates, the potential energy is of the form $V(q(t), t)$. Having these energies in a system with forces which are conservative, the equations of motion may be represented as

$$
\frac{\mathrm{d}}{\mathrm{d} t}\left(\frac{\partial \mathcal{L}}{\partial \dot{q}_{j}}\right)-\frac{\partial \mathcal{L}}{\partial q_{j}}=0, \quad j=1, \ldots, n .
$$

Here $\mathcal{L}(q, \dot{q}, t):=T(q, \dot{q}, t)-V(q, t)$ is a function known as the Lagrangian. The system of equations at (3.1.16) are known as Lagrange's equations.

As mentioned in [51, Chapter 1], equation (3.1.16) may be interpreted as a set of $n$ second-order ordinary differential equations for $n$ unknown functions $q_{j}$, $j=1, \ldots, n$. Hence when incorporating $2 n$ initial values $q\left(t_{0}\right)=q_{0}$ and $\dot{q}\left(t_{0}\right)=\dot{q}_{0}$ it may solved as an initial value problem similar to (3.1.7).

There is a further equivalent approach of solving the equations of motion. For each generalised coordinate $q_{j}$ one can define the corresponding generalised momentum $p_{j}: I \longrightarrow \mathbb{R}$ at $t \in I$ as

$$
p_{j}(t)=\frac{\partial \mathcal{L}}{\partial q_{j}}(q(t), \dot{q}(t), t), \quad j=1, \ldots, n .
$$

The coordinates $p_{j}$ are also called the conjugate momenta. As with the generalised coordinates we will consider the momenta as points $p(t)=\left(p_{1}(t), \ldots, p_{n}(t)\right)$ in an $n$-dimensional space. Taking the Cartesian product of the generalised momenta with the generalised coordinates gives the $2 n$-dimensional space of points 
$(q, p) \in \mathbb{R}^{2 n}$ known as the phase space. Following [27, Chapter 14] a geometrical interpretation is that as time changes, the coordinates $q_{j}$ and $p_{j}$ change tracing a path throughout the phase space similar to how position is traced within the configuration space. With this picture in mind, if we can find any point along this traced path, then the system would be considered as completely determined. The process of finding these points is what we refer to as "studying the dynamics" of the system.

Solving Lagrange's equations can be shown to be equivalent to solving a further system of first-order differential equations

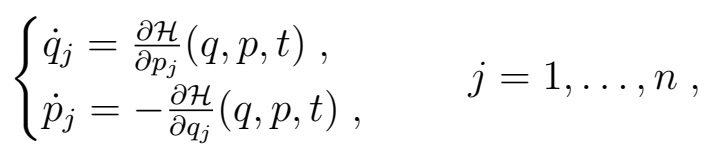

where $\mathcal{H}(q, p, t)$ is a function known as the Hamiltonian. The Hamiltonian is obtained through the partial Legendre transform of the Lagrangian with respect to $\dot{q}$. The equations contained in the above system are known as Hamilton's equations. Referring again to [51, Chapter 7], the above system may be interpreted as a system of $2 n$ first-order ordinary differential equations for $2 n$ unknown functions $q_{j}$ and $p_{j}$. Incorporating the initial conditions $q\left(t_{0}\right)=q_{0}$ and $p\left(t_{0}\right)=p_{0}$ the system may be solved as an initial value problem similar to the equations of motion (3.1.7) and Lagrange's equations (3.1.16). Through this motion may be determined uniquely by Hamilton's equations.

When it comes to solving a system of equations such as Lagrange's or Hamilton's equations, conservation laws are useful. As at [51, Chapter 2], they are of the form of constant valued functions of the generalised coordinates known as integrals of the motion. Their benefit is that they simplify the equations needed to be solved to determine the motion.

An example of an integral of the motion is the conservation of energy (see also [27, Chapters 12 and 14]). This is a constant $E$ which is given by the sum of the kinetic and potential energies:

$$
E=T+V .
$$

This constant is also is referred to as the total energy of the system.

If the Hamiltonian is independent of time then it itself has a conserved value and is an integral of the motion. In fact, further conditions may induce the value of the Hamiltonian to coincide with $E$ as at (3.1.19), i.e. $E$ is determined by the initial values through $\mathcal{H}(q(t), p(t))=\mathcal{H}\left(q_{0}, p_{0}\right)=E$.

Further integrals may also arise if the Lagrangian or the Hamiltonian are independent of a collection of the generalised coordinates $q_{j}, j \in\{1, \ldots, n\}$. These coordinates are referred to as cyclic and induce the conservation of the corresponding conjugate momenta $p_{j}$. 
Lastly, it is possible to derive Lagrange's and Hamilton's equations from the perspective of the calculus of variations. A later section will give an overview of how this ties with classical mechanics hence we do not give further details here. However we mention it here so overall this section as a whole serves as a complete organised list of elements of classical mechanics that we will refer to as the reader progresses through the chapter.

\subsection{Hamilton's and Lagrange's Equations Associ- ated with some Negative Definite Symbols}

We would now like to use the elements of classical mechanics revised in the previous section to study the dynamics of (3.1). We choose to read it as a Hamiltonian corresponding to a mechanical system. In this case we will refer to (3.1) as the Hamilton function to distinguish it from the Hamiltonians in quantum mechanics.

We list below assumptions and properties we now assume when thinking of (3.1) as a Hamiltonian:

(i) We assume that $\mathcal{H} \in C^{1}\left(\mathbb{R}^{2 n}\right)$ defined as

$$
\mathcal{H}(q, p):=\psi(p)+V(q)
$$

is convex with respect to $p$. In particular we require that $\psi \in C N\left(\mathbb{R}^{n}\right) \cap$ $C^{1}\left(\mathbb{R}^{n}\right)$ is convex and coercive as well as $V \in C^{1}\left(\mathbb{R}^{n}\right)$. As previously mentioned, we will look at examples wich induce singularities. This will affect the domain we have to consider for $\mathcal{H}$. However in moving forward, we will in general write $\mathcal{H} \in C^{1}\left(\mathbb{R}^{2 n}\right)$. But, as we reach situations with singularities in the problem, the necessary conditions we have to take so that the examples are well-defined will always be clearly referred to and specified.

Differentiability is important as we want to be able to study the corresponding equations of motion, i.e. Hamilton's equations. We require convexity as we will want to study the corresponding Lagrangian to (3.2.1) which requires finding its Legendre transform.

(ii) The Hamilton function is defined on a $2 n$-dimensional space of points $(q, p) \in$ $\mathbb{R}^{2 n}$ where the variables $q$ and $p$ correspond to the generalised coordinates and momenta respectively. For convenience we refer to this space also as the phase space.

(iii) For $I \subset \mathbb{R}$ each variable $q$ and $p$ may be viewed as mappings in $C^{2}\left(I, \mathbb{R}^{n}\right)$. 
(iv) The Hamilton function has a corresponding system of Hamilton's equations

$$
\left\{\begin{array}{l}
\dot{q}_{j}=\frac{\partial \mathcal{H}}{\partial p_{j}}(q, p)=\psi_{p_{j}}(p), \\
\dot{p}_{j}=-\frac{\partial \mathcal{H}}{\partial q_{j}}(q, p)=-V_{q_{j}}(q), \quad j=1, \ldots, n .
\end{array}\right.
$$

(v) We assume the existence of initial values $q\left(t_{0}\right)=q_{0}$ and $p\left(t_{0}\right)=p_{0}$, where $t_{0}$, $q_{0}$ and $p_{0}$ are fixed. The system of first-order differential equations (3.2.2) is then considered as an initial-value problem.

We would like the corresponding Lagrangian to the Hamilton function. We follow mechanics and calculate the partial Legendre transform of (3.2.1) following Definition 1.9, the convexity of (3.2.1) allows us to do this.

Theorem 3.2.1. The partial Legendre transform of (3.2.1) with respect to $p$ is welldefined giving the function $\mathcal{L}: \mathbb{R}^{2 n} \longrightarrow \mathbb{R}$

$$
\mathcal{L}(q, \eta)=\psi^{*}(\eta)-V(q)
$$

where $\psi^{*}$ is the conjugate function of $\psi$. The function $\mathcal{L}$ is convex with respect to $\eta$. Proof. Taking the partial Legendre transform of (3.2.1) with the respect to $p$ we have

$$
\mathcal{L}(q, \eta)=\sup _{p \in \mathbb{R}^{n}}(\eta \cdot p-\psi(p)-V(q))
$$

for $\eta \in \mathbb{R}^{n}$. The above supremum is achieved when

$$
0=\frac{\partial}{\partial p_{j}}(\eta \cdot p-\psi(p)), \quad j=1, \ldots, n
$$

But (3.2.5) is equivalent to achieving the supremum of

$$
\sup _{p \in \mathbb{R}^{n}}(\eta \cdot p-\psi(p))-V(q) \text {. }
$$

Comparing to Definition 1.9 the first term in (3.2.6) is the full Legendre transform of $\psi$. Therefore (3.2.4) may be rewritten as

$$
\mathcal{L}(q, \eta)=\psi^{*}(\eta)-V(q)
$$

The convexity of $\psi$ guarantees that $\psi^{*}$ is convex by Proposition 1.11. Following Proposition 1.13 the coercivity of $\psi$ gives $\psi^{*}$ to be well-defined. 
Similar to what was done with the Hamilton function we refer to $\mathcal{L}(q, \eta)$ as the Lagrange function to avoid any confusion with Lagrangians from classical mechanics.

An observation is that (3.2.5) gives

$$
\eta_{j}=\psi_{p_{j}}(p)
$$

for $j=1, \ldots, n$. Combining (3.2.7) with Hamilton's equations (3.2.2) confirms that each $\eta_{j}$ is the derivative $\dot{q}_{j}$. This reflects the analogous property in classical mechanics where as well as being dependent on the generalised coordinates, the Lagrangian also depends on the generalised velocities.

Remark 3.2.2. As Proposition 1.11 would suggest the partial Legendre transform of (3.2.3) with respect to $\dot{q}$ gives back the Hamilton function (3.2.1). In doing this we find an alternate representation of $\mathcal{H}$ :

$$
\mathcal{H}(q, p)=\eta \cdot p-\mathcal{L}(q, \eta)
$$

whenever

$$
p_{j}=\psi_{\eta_{j}}^{*}(\eta), \quad j=1, \ldots, n .
$$

Comparing (3.2.9) to (3.2.3) we can rewrite it as

$$
p_{j}=\frac{\partial \mathcal{L}}{\partial \eta_{j}}
$$

confirming that the coordinates $p_{j}$ correspond to the conjugate momenta in classical mechanics (recall (3.1.17)).

With (3.2.3) we can show that solving Hamilton's equations at (3.2.2) is equivalent to solving an additional system of equations:

Theorem 3.2.3. Let $\mathcal{H}$ be the Hamilton function (3.2.1) with $\mathcal{L}$ being the corresponding Lagrange function (3.2.3) such that $\psi^{*} \in C^{2}\left(\mathbb{R}^{n}\right)$, i.e. $\mathcal{L}(., \eta) \in C^{1}\left(\mathbb{R}^{n}\right)$ and $\mathcal{L}(q,.) \in C^{2}\left(\mathbb{R}^{n}\right)$. Then solving the system of Hamilton's equations (3.2.2) is equivalent to solving the following system of $n$ second-order ordinary differential equations:

$$
\frac{\mathrm{d}}{\mathrm{d} t}\left(\psi_{\eta_{j}}^{*}(\eta)\right)=-V_{q_{j}}(q), \quad j=1, \ldots, n .
$$

Equations (3.2.11) are Lagrange's equations for (3.2.3). Further

$$
\frac{\partial \mathcal{H}}{\partial q_{j}}=-\frac{\partial \mathcal{L}}{\partial q_{j}}, \quad j=1, \ldots, n
$$


Proof. We first prove that the existence of Hamilton's equations implies (3.2.11) to be true. Following this the converse can then be proved analogously to overall give the equivalence between the two sets of equations.

The first implication depends on comparing two alternative forms of expressing the differential of the Lagrange function with respect to $t$. We denote this differential by $\mathrm{d} \mathcal{L}$. We take inspiration from a similar approach used to derive Hamilton's equations in [26, pages 337 to 338 ].

Using the chain rule, the differential of $\mathcal{L}(q(t), \dot{q}(t))$ with respect to $t$ gives the first form:

$$
\mathrm{d} \mathcal{L}=\sum_{j=1}^{n} \psi_{\eta_{j}}^{*}(\eta) \mathrm{d} \eta_{i}-\sum_{j=1}^{n} V_{q_{j}}(q) \mathrm{d} q_{j} .
$$

To obtain the second form, we recollect from Theorem 3.2.1 that whenever

$$
\eta_{j}=\psi_{p_{j}}(p), \quad j=1, \ldots, n,
$$

$\mathcal{L}$ has an alternate representation:

$$
\mathcal{L}(q, \eta)=\eta \cdot p-\mathcal{H}(q, p)
$$

Taking the differential of $\mathcal{L}$ with respect to $t$ again but with the above form in mind gives

$$
\mathrm{d} \mathcal{L}=\sum_{j=1}^{n} p_{j} \mathrm{~d} \eta_{j}+\sum_{j=1}^{n} \eta_{j} \mathrm{~d} p_{j}-\sum_{j=1}^{n} \psi_{p_{j}}(p) \mathrm{d} p_{j}-\sum_{j=1}^{n} V_{q_{j}}(q) \mathrm{d} q_{j}
$$

Combining (3.2.14) with (3.2.16) we obtain the second form of $\mathrm{d} \mathcal{L}$ :

$$
\mathrm{d} \mathcal{L}=\sum_{j=1}^{n} p_{j} \mathrm{~d} \eta_{j}-\sum_{j=1}^{n} V_{q_{j}}(q) \mathrm{d} q_{j}
$$

Comparing the coefficients of $\mathrm{d} \eta_{i}$ and $\mathrm{d} q_{i}$ in (3.2.13) to those at (3.2.17) we deduce that

$$
p_{j}=\psi_{\eta_{j}}^{*}(\eta)
$$

and

$$
V_{q_{j}}(q)=V_{q_{j}}(q) \text {. }
$$

Note, (3.2.19) is equivalent to writing

$$
\frac{\partial \mathcal{H}}{\partial q_{j}}=-\frac{\partial \mathcal{L}}{\partial q_{j}}, \quad j=1, \ldots, n .
$$


But, recall from the second equation in Hamilton's equations it holds that $\dot{p}_{i}=$ $-V_{q_{i}}(q)$. Combining this with equation (3.2.18) gives Lagrange's equations:

$$
\frac{\mathrm{d}}{\mathrm{d} t}\left(\psi_{\eta_{j}}^{*}(\eta)\right)=-V_{q_{j}}(q)
$$

The converse is proved in an analogous way: we compare two forms of the differential of the Hamilton function with respect to $t$, denoted $\mathrm{d} \mathcal{H}$. The first form is given by calculating $\mathrm{d} \mathcal{H}$ directly from $\mathcal{H}(q(t), p(t))$, similar to what was done for $\mathcal{L}$ to obtain (3.2.13). For the second form we recollect the other form of the Hamilton function at (3.2.8) which holds whenever (3.2.18) is true. Using this to recalculate $\mathrm{d} \mathcal{H}$ followed by incorporating (3.2.18) and Lagrange's equations gives the second expression of $\mathrm{d} \mathcal{H}$. Comparing terms then completes the proof. be

Using the chain rule Lagrange's equations may be calculated more explicitly to

$$
\sum_{l=1}^{n} \psi_{\dot{q}_{l} \dot{q}_{j}}^{*}(\dot{q}) \ddot{q}_{l}=-V_{q_{j}}(q), \quad j=1, \ldots, n .
$$

It is important to note that we required some more differentiability with $\psi^{*} \in$ $C^{2}\left(\mathbb{R}^{n}\right)$ to ensure the possibility of forming Lagrange's equations. However, it is possible to obtain this extra smoothness without imposing such extra conditions in certain examples:

Example 3.2.4. We want to study the dynamics associated with the Hamilton function

$$
\mathcal{H}(q, p)=\frac{1}{\alpha}\|p\|^{\alpha}+V(q),
$$

for $1<\alpha \leq 2$. Here $\psi(p):=\psi_{\alpha}(p)=\frac{1}{\alpha}\|p\|^{\alpha}$, a function we know to be continuous negative definite from Example 2.22.A. It holds that $\psi \in C^{1}\left(\mathbb{R}^{n}\right)$ and we know from Examples 1.8.A and 1.14.A that $\psi$ is convex and coercive respectively. Hence $\mathcal{H} \in C^{1}\left(\mathbb{R}^{2 n}\right)$ at (3.2.23) satisfies all that we require of a Hamilton function.

Using (3.2.2) Hamilton's equations follow as

$$
\left\{\begin{array}{l}
\dot{q}_{j}=p_{j}\|p\|^{\alpha-2} \\
\dot{p}_{j}=-V_{q_{j}}(q)
\end{array}\right.
$$

By Theorem 3.2.1 the corresponding Lagrange function follows as

$$
\mathcal{L}(q, \eta)=\frac{1}{\alpha^{*}}\|\eta\|^{\alpha^{*}}-V(q)
$$


where from Example 1.17 we recall that $\psi_{\alpha}^{*}(\eta)=\frac{1}{\alpha^{*}}\|\eta\|^{\alpha^{*}}$. Important to note here is that $\psi_{\alpha}^{*} \in C^{2}\left(\mathbb{R}^{2}\right)$ as $1<\alpha \leq 2$ implies that $\alpha^{*} \geq 2$. This means that Lagrange's equations are well-defined and follow as

$$
\frac{\mathrm{d}}{\mathrm{d} t}\left(\eta_{j}\|\eta\|^{\alpha^{*}-2}\right)=-V_{q_{j}}(q) .
$$

Hence despite not initially assuming that $\psi_{\alpha}^{*}$ would be $C^{2}$, we were able to gain the required differentiability regardless. Therefore, although the extra conditions of Theorem 3.2.3 are ones we cannot ignore, they may not always be essential for some examples of Hamilton and Lagrange functions.

\subsection{Conservation Laws}

Recall we discussed at the end of Section 3.1 that problems in classical mechanics can be made simpler to solve by exploiting integrals of the system, i.e. conserved quantites associated with the problem. We would like to have similar advantages when dealing with the Hamilton function (3.2.1).

First, let us consider what is induced when the system is invariant under a translation of $t \in I$, i.e. $t \mapsto t+t^{*} \in I$. In mechanics this leads to the conservation of energy and hence the value of the Hamiltonian to be conserved. Being always independent of $t$, we therefore would expect the value of the Hamilton function to also be conserved.

For convenience we follow [27] and call the Hamilton function autonomous due to its invariance with respect to $t$. The same term will be used for the Lagrange function or any other expression should they also have this invariance.

We begin with proving the conservation of the Hamilton function. The ideas to follow are taken from [51, Chapter 2].

Theorem 3.3.1. The value of the Hamilton function

$$
\mathcal{H}(q, p)=\psi(p)+V(q),
$$

where $\psi \in C N\left(\mathbb{R}^{n}\right) \cap C^{1}\left(\mathbb{R}^{n}\right)$ and $V \in C^{1}\left(\mathbb{R}^{n}\right)$ is a conserved quantity. We denote this by $E$. This means for a solution $(q(t), p(t))$ of Hamilton's equations with initial conditions $\left(q\left(t_{0}\right), p\left(t_{0}\right)\right)=\left(q_{0}, p_{0}\right), E$ is determined by $\mathcal{H}(q(t), p(t))=\mathcal{H}\left(q_{0}, p_{0}\right)=E$ for all $t$.

Proof. Using the chain rule to calculate the derivative of (3.3.1) with respect to $t$, we have

$$
\frac{\mathrm{d} \mathcal{H}}{\mathrm{d} t}=\sum_{j=1}^{n} V_{q_{j}} \dot{q}_{j}+\sum_{j=1}^{n} \psi_{p_{j}} \dot{p}_{j}
$$




$$
=\sum_{j=1}^{n} V_{q_{j}} \cdot\left(\psi_{p_{j}}\right)+\sum_{j=1}^{n} \psi_{p_{j}} \cdot\left(-V_{q_{j}}\right)=0,
$$

where we have used Hamilton's equations.

There is a further interpretation of $E$ : we know from Remark 3.2.2 that the Hamilton function (3.3.1) is equal to the partial Legendre transform of the Lagrange function. Hence, using this with Theorem 3.3.1 we may equivalently write

$$
\frac{\mathrm{d} \mathcal{H}}{\mathrm{d} t}=\frac{\mathrm{d}}{\mathrm{d} t}\left(\sum_{j=1}^{n} \psi_{\eta_{j}}^{*}(\eta) \eta_{j}-\mathcal{L}(q, \eta)\right)=0
$$

where we recall that $p_{j}=\psi_{\eta_{j}}^{*}(\eta)$ from (3.2.9). Looking at what is contained in the parantheses above, we have proved

Theorem 3.3.2. The expression

$$
\sum_{j=1}^{n} \psi_{\eta_{j}}^{*}(\eta) \eta_{j}-\mathcal{L}(q, \eta)
$$

is a conserved value equal to that of the Hamilton function.

The above expression corresponds to the energy for a mechanical system and is used in classical mechanics to derive properties such as the conservation of energy.

In classical mechanics the energy is usually derived from the perspective of the Lagrangian. However we have started with the Hamilton function. We can also derive (3.3.3) using the Lagrange function

$$
\mathcal{L}(q, \dot{q})=\psi^{*}(\dot{q})-V(q),
$$

$\mathcal{L}(., \eta) \in C^{1}\left(\mathbb{R}^{n}\right)$ and $\mathcal{L}(q,.) \in C^{2}\left(\mathbb{R}^{n}\right)$. The derivative of (3.3.4) with respect to $t$ gives

$$
\frac{\mathrm{d} \mathcal{L}}{\mathrm{d} t}=-\sum_{j=1}^{n} V_{q_{j}} \dot{q}_{j}+\sum_{j=1}^{n} \psi_{\dot{q}_{j}}^{*} \ddot{q}_{j} .
$$

Using Lagrange's equations and then the product rule of differentiation we can write

$$
\begin{aligned}
\frac{\mathrm{d} \mathcal{L}}{\mathrm{d} t} & =\sum_{j=1}^{n} \frac{\mathrm{d}}{\mathrm{d} t}\left(\psi_{\dot{q}_{j}}^{*}\right) \dot{q}_{j}+\sum_{j=1}^{n} \psi_{\dot{q}_{j}}^{*} \ddot{q}_{i} \\
& =\sum_{j=1}^{n} \frac{\mathrm{d}}{\mathrm{d} t}\left(\psi_{\dot{q}_{j}}^{*} \dot{q}_{j}\right) .
\end{aligned}
$$


Rearranging we have

$$
\frac{\mathrm{d}}{\mathrm{d} t}\left(\sum_{j=1}^{n} \psi_{\dot{q}_{j}}^{*}(\dot{q}) \dot{q}_{j}-\mathcal{L}(q, \dot{q})\right)=0
$$

giving the claim of Theorem 3.3.2.

We can take this analogue of a "conserved energy" further by recollecting about the total energy at (3.1.19). We do this with the help of [27, Chapter 12 and 14]. Whenever independent of time, it is possible for the conserved Hamiltonian in classical mechanics to be of the form

$$
\mathcal{H}(q, p)=T(q, p)+V(q)
$$

the total energy conserved. Here $T$ is the total kinetic energy but unlike before, now expressed in terms of $q$ and $p$, and $V$ is the potential energy of the system as before. We notice that the Hamilton function, also with a conserved value, has the form of (3.3.6). Hence for our study we choose to read the Hamilton function as the sum of $\psi(p)=T(p)$, the kinetic (energy) term, and $V(q)$ the potential (energy) term. However, as seen at [51, Chapter 2], Hamiltonians such as (3.3.6) are obtained through the Legendre transform of Lagrangians

$$
\mathcal{L}(q, \eta)=T(q, \eta)-V(q)
$$

where $T$ is a quadratic form with respect to $\eta$. Hence to maintain the analogue should we not expect the Lagrange function to also be of the form of (3.3.7)? I.e. should the Lagrange function (3.3.4) be the kinetic term minus the potential term? From Theorem 3.2.1 we know the true form of the Lagrange function to be

$$
\mathcal{L}(q, \eta)=T^{*}(\eta)-V(q)
$$

where $T^{*}$ is the conjugate of the kinetic term $T$. Comparing (3.3.8) to (3.3.7), (3.3.8) suggests the kinetic term to be $T^{*}$. But we have already established $T(p)$, a different term, as the kinetic term.

The problem is that $\psi(p)=T(p)$ is a continuous negative definite function which does not necessarily have the same values as its conjuagte $T^{*}(\eta)$. Hence the Lagrange function (3.3.4) does not always have the form of (3.3.7) as seen in classical mechanics. To reflect this distinction we will call $T^{*}$ in the Lagrange function the dual kinetic (energy) term to $T$.

The case from classical mechanics case is not lost however. One could look at our analogue as its generalisation. We know from Example 2.4.C that all positive semidefinite quadratic forms are elements of the set of all continuous negative functions. Additionally values of quadratic forms and their Legendre transforms coincide as 
explained at [2, page 66] (or at least they coincide up to a constant). Therefore whenever $\psi \in C N\left(\mathbb{R}^{n}\right)$ is a quadratic form, it holds that $\psi=\psi^{*}$ and the Lagrange function (3.3.4) would be of the form

$$
\mathcal{L}(q, \eta)=T(\eta)-V(q)
$$

giving (3.3.7) as in classical mechanics.

Example 3.3.3. Recall from Example 3.2.4 the Hamilton function

$$
\mathcal{H}(q, p)=\frac{1}{\alpha}\|p\|^{\alpha}+V(q)
$$

and its Lagrange function

$$
\mathcal{L}(q, \eta)=\frac{1}{\alpha^{*}}\|\eta\|^{\alpha^{*}}-V(q)
$$

The kinetic and potential terms of (3.3.10) are $\frac{1}{\alpha}\|p\|^{\alpha}$ and $V(q)$ respectively. The dual kinetic term at (3.3.11) is $\frac{1}{\alpha^{*}}\|\eta\|^{\alpha^{*}}$. Notice the kinetic term is not necessarily of a quadratic form. Hence the dual kinetic term $\frac{1}{\alpha^{*}}\|\cdot\|^{\alpha^{*}}$ of (3.3.11) of course does not always have equal values. However, we see that (3.3.10) and (3.3.11) have the expected forms of (3.3.6) and (3.3.8).

We now want to develop cyclic coordinates but for the Hamilton and Lagrange function. We do this following [27, Chapters 12 and 14].

Definition 3.3.4. Any coordinate $q_{j}, j \in\{1, \ldots, n\}$, that does not appear explicitly in the Hamilton function is called cyclic.

In classical mechanics the conjugate momenta for cyclic generalised coordinates are constant. This result extends to our new situation:

Theorem 3.3.5. Suppose the Hamilton function has a cyclic coordinate $q_{j}, j \in$ $\{1, \ldots, n\}$. Then the corresponding coordinate $p_{j}$ is constant.

Proof. Suppose $q_{j}$ is cyclic. Then by Hamilton's equations it holds that

$$
\dot{p}_{j}=-\frac{\partial \mathcal{H}}{\partial q_{j}}=0
$$

Hence $p_{j}$ must be constant. 
Recognising a cyclic coordinate in the Lagrange function is in fact equivalent to finding cyclic coordinates in the Hamilton function due to the connection between the two functions as shown at Theorem 3.2.3:

$$
\frac{\partial \mathcal{H}}{\partial q_{j}}=-\frac{\partial \mathcal{L}}{\partial q_{j}}, \quad j=1, \ldots, n .
$$

Recall in this theorem it was assumed that for $q$ and $\eta$ fixed, $\mathcal{L}(., \eta) \in C^{1}\left(\mathbb{R}^{n}\right)$ and $\mathcal{L}(q,.) \in C^{2}\left(\mathbb{R}^{n}\right)$. Therefore, if a coordinate was cyclic in the Hamilton function then the left-hand side of (3.3.13) would be zero. When compared with the righthand side this shows that the Lagrange function must also be independent of the same coordinate. One can argue in a similar way to prove the converse. A similar observation is made with Hamiltonians and Lagrangians at [27, page 406].

An analogous result to Theorem 3.3.5 follows for the Lagrange function. Let us call coordinates $q_{j}$ who do not appear in the Lagrange function also cyclic. Then we have:

Theorem 3.3.6. Suppose the Lagrange function has a cyclic coordinate $q_{j}, j \in$ $\{1, \ldots, n\}$. Then the corresponding coordinate $p_{j}$ is constant.

Proof. Recall from (3.2.9) we have

$$
p_{j}=\psi_{\eta_{j}}^{*}(\eta) .
$$

Differentiating both sides of the above with respect to $t$ and using Lagrange's equations we have

$$
\dot{p}_{j}=\frac{\partial \mathcal{L}}{\partial q_{j}}=-V_{q_{j}}(q) .
$$

But, the Lagrange function is assumed to be independent of $q_{j}$. Hence, equation (3.3.15) is equal to 0 and so $p_{j}$ is constant.

One can think of recognising cyclic coordinates as spotting symmetries in the system. In this way we can form a generalisation of our approach with cyclic coordinates to find more conserved quantities. Such a result exists in classical mechanics known as Noether's Theorem. Following [2, pages 20 to 21, and 88] and [27, Chapter 12 ] we want to develop an analogous result. To begin we need

Definition 3.3.7. A family of diffeomorphisms $Q^{s}: \mathbb{R}^{n} \longrightarrow \mathbb{R}^{n}, s \in \mathbb{R}$, is said to form a one-parameter group if

(i) $Q^{0}(x)=x$ (the identity mapping),

(ii) $Q^{t+s}(x)=\left(Q^{t} \circ Q^{s}\right)(x)$, 
(iii) $\left(Q^{-t} \circ Q^{t}\right)(x)=x\left(Q^{-t}\right.$ is the inverse of $\left.Q^{t}\right)$.

Take a one-parameter group of diffeomorphisms $Q^{s}, s \in \mathbb{R}$, defined on the configuration space of points $q$, i.e. $Q^{s}: \mathbb{R}^{n} \longrightarrow \mathbb{R}^{n}, q \mapsto Q^{s}(q)=\left(Q_{1}^{s}(q), \ldots, Q_{n}^{s}(q)\right)$. The function $Q^{s}$ may also be thought of as a function of two variables, $q$ and $s$. These functions will represent symmetric invariances in our dynamics, for example a translation in a particular coordinate. Through this we define:

Definition 3.3.8. Let $Q^{s}$ be a one-paramter group of diffeomorphisms defined on the configuration space of points $q \in \mathbb{R}^{n}$. Let $\mathcal{L}$ be the Lagrange function. Then we say that $\mathcal{L}$ admits the mappings $Q^{s}$ if

$$
\mathcal{L}(q, \dot{q})=\mathcal{L}\left(Q^{s}(q), \dot{Q}^{s}(q, \dot{q})\right)
$$

Looking at (3.3.16), we need to understand what is meant by $\dot{Q}^{s}(q, \dot{q})$. We consider it as the derivative of $Q^{s}(q)$ with respect to $t$ :

$$
\dot{Q}^{s}(q, \dot{q})=\sum_{j=1}^{n} \frac{\partial Q^{s}}{\partial q_{j}} \dot{q}_{j} .
$$

Through this the function $\dot{Q}^{s}$ may be considered as a function of three variables, $q$, $\dot{q}$ and $s$. Further it shares a similar identity property to $Q^{s}$, i.e. $\dot{Q}^{0}(q, \dot{q})=\dot{q}$. Now we are ready to give our analogue to Noether's theorem:

Theorem 3.3.9. Let $\mathcal{L}$ be the Lagrange function such that $\mathcal{L}(., \eta) \in C^{1}\left(\mathbb{R}^{n}\right)$ and $\mathcal{L}(q,.) \in C^{2}\left(\mathbb{R}^{n}\right)$. Further suppose $\mathcal{L}$ admits a one-paramter group of diffeomorphisms $Q^{s}$. Then

$$
I(q, \dot{q})=\left.\sum_{j=1}^{n} \psi_{\dot{q}_{j}}^{*} \frac{\partial Q_{j}^{s}}{\partial s}\right|_{s=0}
$$

is constant.

Proof. For the proof we refer to [27, Chapter 12] where the same result is proved for classical Lagrangians. Since $\mathcal{L}(., \eta) \in C^{1}\left(\mathbb{R}^{n}\right)$ and $\mathcal{L}(q,.) \in C^{2}\left(\mathbb{R}^{n}\right)$, the Lagrange function $\mathcal{L}$ satisfies what is needed so that the proof may be completed analogously to what is done there.

The result of Theorem 3.3.9 acts as a generalisation of finding constants induced by cyclic coordinates. This is demonstrated in the next example:

Example 3.3.10. Suppose the $j$ th coordinate $q_{j}$ is cyclic in the Lagrange function. Therefore any translation in this coordinate would leave $\mathcal{L}$ invariant. Hence let us define the diffeomorphisms $Q^{s}: \mathbb{R}^{n} \longrightarrow \mathbb{R}^{n}$ on the configuration space as:

$$
Q^{s}(q)=\left(Q_{1}^{s}(q), \ldots, Q_{j}^{s}(q), \ldots, Q_{n}^{s}(q)\right)
$$




$$
:=\left(q_{1}, \ldots, q_{j}+s, \ldots, q_{n}\right)
$$

where the $j$ th coordinate $q_{j}$ has been translated by $s \in \mathbb{R}$. Because $q_{j}$ is cyclic the Lagrange function admits the above mappings:

$$
\begin{aligned}
\mathcal{L}(q, \dot{q}) & =\mathcal{L}\left(q_{1}, \ldots, q_{j}+s, \ldots, q_{n}, \dot{q}\right) \\
& =\mathcal{L}\left(Q^{s}(q), \dot{Q}^{s}(q, \dot{q})\right) .
\end{aligned}
$$

Using Theorem 3.3 .9 a constant is then given by

$$
I(q, \dot{q})=\left.\sum_{j=1}^{n} \psi_{\dot{q}_{j}}^{*} \frac{\partial Q_{j}^{s}}{\partial s}\right|_{s=0} .
$$

Explicitly calculating (3.3.19) and recollecting (3.3.14), we have

$$
I(q, \dot{q})=p_{j} .
$$

Hence we have the claim of Theorem 3.3.6. 


\subsection{Relations to the Calculus of Variations}

In classical mechanics it is possible to determine the motion of a mechanical system using the calculus of variations. A source for seeing this is at [10, Chapter 1]. It requires the minimising of variational integrals (or action functionals as they are named in [27, Chapter 13]), i.e. functionals of the form

$$
F(u)=\int_{I} \mathcal{F}(t, u(t), \dot{u}(t)) \mathrm{d} t
$$

where $I=(a, b) \subset \mathbb{R}$ is a bounded interval, $u \in C\left(\bar{I}, \mathbb{R}^{n}\right)$ is sufficiently smooth, and $\mathcal{F} \in C^{2}(\mathcal{U})$. The set $\mathcal{U} \subset \mathbb{R}^{2 n+1}$ is a neighbourhood containing

$$
\{(t, u(t), \dot{u}(t)) \mid t \in \bar{I}\} \subset \mathbb{R}^{2 n+1},
$$

the 1-graph of $u$. By "minimising" we mean to determine mappings $u$ that give the minimum (or at least a stationary) value of (3.4.1).

Given $u, \mathcal{F}(v)$ is well-defined for any $v \in C^{1}\left(\bar{I}, \mathbb{R}^{n}\right)$ if $\|v-u\|_{C^{1}(I)}<\delta$ for $\delta>0$ sufficiently small. Here $\|\cdot\|_{C^{1}(I)}$ is a norm such that for $v \in C^{1}\left(\bar{I}, \mathbb{R}^{n}\right)$

$$
\|v\|_{C^{1}(I)}:=\sup _{a \leq t \leq b}|v(t)|+\sup _{a \leq t \leq b}|\dot{v}(t)| .
$$

A way to minimise (3.4.1) is to find extremals who solve the corresponding EulerLagrange equations

$$
\frac{\mathrm{d}}{\mathrm{d} t}\left(\frac{\partial \mathcal{F}}{\partial \dot{u}_{j}}\right)=\frac{\partial \mathcal{F}}{\partial u_{j}}, \quad j=1 \ldots, n,
$$

a system of second-order ordinary differential equations. An important point is that extremals or solutions of (3.4.3) are not necessarily minimisers. However there are ways of determining whether a given extremal is a minimiser or not. For more details we refer to [10].

Nevertheless, the Euler-Lagrange equations are relevant to dynamics due to their structure. Taking $\mathcal{F}$ as a Lagrangian, equations (3.4.3) become Lagrange's equations. Hence determining the motion of a mechanical system is equivalent to solving for extremals.

We would like to know more about action functionals related to Hamilton and Lagrange functions, in particular to obtain a similar equivalence between finding extremals and solving our Lagrange's equations. We will see that having such a connection to the calculus of variations will prove to be useful for later chapters.

The Lagrange function and the mappings $q$ satisfy what is sufficient for them to play the roles of $\mathcal{F}$ and $u$ respectively. Hence with the help of [10, Chapter 1] we may collect the above results but for the Lagrange function and $q$. The proofs 
follow analogously to those as seen at [10]. With all of this having been said, let us reset the problem:

Consider $q \in C^{2}\left(\bar{I}, \mathbb{R}^{n}\right), I \subset \mathbb{R}$, and the Lagrange function

$$
\mathcal{L}(q, \dot{q})=\psi^{*}(\dot{q})-V(q) .
$$

For $q$ and $\dot{q}$ fixed we have that $\mathcal{L}(., \dot{q}) \in C^{1}(\mathcal{U})$ and $\mathcal{L}(q,.) \in C^{2}(\mathcal{V})$ where $\mathcal{U} \times \mathcal{V}$ form an open neighbourhood of

$$
\{(t, q(t), \dot{q}(t)) \mid t \in \bar{I}\} \subset \mathbb{R}^{2 n+1},
$$

the 1-graph of $q$. The aim is to find minimisers of the functional

$$
L(q)=\int_{I}\left[\psi^{*}(\dot{q}(t))-V(q(t))\right] \mathrm{d} t .
$$

Note given $q$, the functional $L$ is defined for $\rho \in C^{1}\left(\bar{I}, \mathbb{R}^{n}\right)$ such that $\|q-\rho\|_{C^{1}(I)}<\delta$, for $\delta>0$ sufficiently small.

We take the interpretation that minimisers should act like stationary values for the functional, i.e. induce the directional derivative $\delta L(q, \varphi):=\left.\frac{\mathrm{d}}{\mathrm{d} \epsilon} L(q+\epsilon \varphi)\right|_{\epsilon=0}$ to vanish in all smooth directions $\varphi \in C_{c}^{\infty}\left(I, \mathbb{R}^{n}\right)$. Let $|\epsilon|<\epsilon_{0}$ for $\epsilon_{0}:=\frac{\delta}{\|\varphi\|_{C^{1}(I)}}$. For $q$ fixed and any $\varphi$, through this $L(q+\epsilon \varphi)$ is well-defined as a function of $\epsilon$, in fact $L(q+(.) \varphi) \in C^{1}\left(-\epsilon_{0}, \epsilon_{0}\right)$.

Definition 3.4.1. Let $q \in C^{1}\left(I, \mathbb{R}^{n}\right)$ satisfy

$$
\delta L(q, \varphi)=\int_{I}\left[\sum_{j=1}^{n}\left(-V_{q_{j}}(q(t))\right) \cdot \varphi_{j}(t)+\sum_{j=1}^{n} \psi_{\dot{q}_{j}}^{*}(\dot{q}(t)) \cdot \dot{\varphi}_{j}(t)\right] \mathrm{d} t=0
$$

for all $\varphi \in C_{c}^{\infty}\left(I, \mathbb{R}^{n}\right), \varphi(t)=\left(\varphi_{1}(t), \ldots, \varphi_{n}(t)\right) \in \mathbb{R}^{n}$. Then we call $q$ a weak extremal of $L$.

The next proposition relates the extremals to the minimisers. First we formally define

Definition 3.4.2. We call $q \in C^{1}\left(\bar{I}, \mathbb{R}^{n}\right)$ a weak minimiser of $L$ if

$$
L(q) \leq L(q+\varphi)
$$

for all $\varphi \in C_{c}^{\infty}\left(I, \mathbb{R}^{n}\right)$ such that $\|\varphi\|_{C^{1}(I)}<\mu, 0<\mu<1$ sufficiently small.

Proposition 3.4.3. A weak minimser of $L$ is a weak extremal of $L$. 
By Proposition 3.4.3 finding extremals are not sufficient for determining minimisers. However, it is the connection between the Euler-Lagrange functions and Lagrange's equations we are more interested in.

Suppose $I=(a, b) \subset \mathbb{R}$. As $\varphi$ is a function defined on $I$ with values in $\mathbb{R}^{n}$, the term $q+\epsilon \varphi$ may be viewed as paths in the space of $q(t)$ plotted against $t$. Even more as $\varphi$ has compact support, these paths are constrained to move from one fixed point $q(a)$ to another $q(b)$. From this geometrical point of view we think of finding extremals as finding the correct path connecting $q(a)$ to $q(b)$ that minimises $L$. A similar picture for extremals is desribed at [26, Chapter 2].

Now we come to the main result:

Proposition 3.4.4. Let $q$ be a weak extremal of L. Then $q$ solves

$$
\frac{\mathrm{d}}{\mathrm{d} t}\left(\psi_{\dot{q}_{j}}^{*}(\dot{q}(t))\right)+V_{q_{j}}(q(t))=0, \quad j=1, \ldots, n .
$$

As in [27, Chapter 13], we will refer to (3.4.6) as the Euler-Lagrange equations. We call solutions of (3.4.6) extremals of $L$.

Notice the equations at (3.4.6) coincide with Lagrange's equations from (3.2.11). Hence solving (3.4.6) is equivalent to finding extremals of $L$.

We recall that weak extremals (or now solutions of (3.4.6)) may be thought of as paths constrained between two fixed points. Through this, Lagrange's equations at (3.4.6) may be viewed as a boundary value problem with conditions $q(a)=q_{a}$ and $q(b)=q_{b}$ for some $q_{a}, q_{b} \in \mathbb{R}^{n}$.

We may also deduce some conservation laws using the calculus of variations:

Proposition 3.4.5. Define $\Phi \in C^{1}\left(\mathbb{R}^{n}\right)$ as

$$
\Phi(q, \dot{q}):=\sum_{j=1}^{n} \psi_{\dot{q}_{j}}^{*}(\dot{q}) \dot{q}_{j}-\mathcal{L}(q, \dot{q}) .
$$

Then for any weak extremal $q \in C^{2}\left(I, \mathbb{R}^{n}\right)$ the function (3.4.7) is constant.

Proof. Using the product and chain rules it can be shown that

$$
\frac{\mathrm{d}}{\mathrm{d} t} \Phi(q(t), \dot{q}(t))=\sum_{j=1}^{n} \dot{q}_{j}\left(\frac{\mathrm{d}}{\mathrm{d} t}\left(\psi_{\dot{q}_{j}}^{*}(\dot{q}(t))\right)+V_{q_{j}}(q(t))\right) .
$$

Following Proposition 3.4.4, whenever $q$ is an extremal the term contained in the parantheses on the right-and side of (3.4.8) must be zero. This gives the desired result. 
Notice (3.4.7) coincides with (3.3.3) at Theorem 3.3.2. Hence Proposition 3.4.5 is equivalent to this theorem, but proved using the calculus of variations. as

In fact, recollecting that $p_{j}=\psi_{\dot{q}_{j}}^{*}$, equation (3.4.7) may alternatively be written

$$
\Phi(q, \dot{q})=\sum_{j=1}^{n} p_{j} \dot{q}_{j}-\mathcal{L}(q, \dot{q})
$$

This is the alternate representation of the Hamilton function at (3.2.8). Hence Proposition 3.4.5 also obtains the result of Theorem 3.3.1.

It is possible to identify the Euler-Lagrange equations as Hamilton's equations. With additional help from [26, Chapter 8.5], this is seen by looking for minimisers of the functional

$$
G(q, p)=\int_{I} \mathcal{G}(q(t), \dot{q}(t), p(t), \dot{p}(t)) \mathrm{d} t
$$

where $q, p \in C^{2}\left(\bar{I}, \mathbb{R}^{n}\right)$ and $\mathcal{G}$ is defined as

$$
\mathcal{G}(q(t), p(t), \dot{q}(t), \dot{p}(t)):=p(t) \cdot \dot{q}(t)-\psi(p(t))-V(q(t)) .
$$

At equation (3.4.10) it is assumed that $p_{j}(t)=\psi_{\dot{q}_{j}}^{*}(\dot{q}(t))$ for $j=1, \ldots, n$. For $(q, p)$ and $(\dot{q}, \dot{p})$ fixed we also take that $\mathcal{G}(., \dot{q}, ., \dot{p}) \in C^{1}(\mathcal{M})$ and $\mathcal{G}(q, ., p,.) \in C^{2}(\mathcal{N})$ where $\mathcal{M} \times \mathcal{N}$ form an open neighbourhood of

$$
\{(t, q(t), p(t), \dot{q}(t), \dot{p}(t)) \mid t \in \bar{I}\} \subset \mathbb{R}^{4 n+1},
$$

the 1-graph of $(q, p)$. Following Proposition 3.4.4 the corresponding Euler-Lagrange equations appear as

$$
\frac{\mathrm{d}}{\mathrm{d} t}\left(\mathcal{G}_{\dot{q}_{j}}\right)-\mathcal{G}_{q_{j}}=0 \text { and } \quad \frac{\mathrm{d}}{\mathrm{d} t}\left(\mathcal{G}_{\dot{p}_{j}}\right)-\mathcal{G}_{p_{j}}=0
$$

or

$$
\dot{p}_{j}+V_{q_{j}}=0 \quad \text { and } \quad \dot{q}_{j}-\psi_{p_{j}}=0,
$$

which are Hamilton's equations from (3.2.2). 


\section{Chapter 4}

\section{Examples of Hamilton and Lagrange Functions}

We want to study the dynamics of the Hamilton system associated with

$$
\mathcal{H}(q, p)=\psi(p)+V(q),
$$

$\psi \in C N\left(\mathbb{R}^{n}\right) \cap C^{1}\left(\mathbb{R}^{n}\right)$ and $V \in C^{1}\left(\mathbb{R}^{n}\right)$, i.e. we try to solve Hamilton's equations:

$$
\left\{\begin{array}{l}
\dot{q}_{j}=\psi_{p_{j}}(p), \\
\dot{p}_{j}=-V_{q_{j}}(q),
\end{array} \quad j=1, \ldots, n .\right.
$$

To do this we construct examples of (4.1) that provide good analogues to wellstudied Hamiltonians in classical mechanics. We know that solutions to Hamilton's equations are equivalent to those obtained from solving Lagrange's equations:

$$
\frac{\mathrm{d}}{\mathrm{d} t}\left(\psi_{\eta_{j}}^{*}(\eta)\right)=-V_{q_{j}}(q)
$$

where the corresponding Lagrange function follows as

$$
\mathcal{L}(q, \eta)=\psi^{*}(\eta)-V(q) .
$$

For $q$ and $\eta$ fixed, recall that $\mathcal{L}(., \eta) \in C^{1}\left(\mathbb{R}^{n}\right)$ and $\mathcal{L}(q,.) \in C^{2}\left(\mathbb{R}^{n}\right)$. Hence the above system of equations (4.3) will also be investigated. Introducing the inital conditions, we recall that these systems may be read as initial-value problems. Once having found the general solutions any constants of integration may then be eliminated using the initial conditions. Being equivalent approaches we of course expect to obtain the same solutions when solving either system.

We will take full advantage of the conservation laws established in Section 3.3. We will see that this reduces the amount of differential equations we need to solve 
for a given problem. For example in the two-dimensional examples with a system of four Hamilton's equations, a conserved value will be incorporated so that we need only consider three differential equations.

However, one clear distinction between the Hamilton and Lagrange approach is that when solving Hamilton's equations, we are looking for two sets of solutions, namely the coordinates $q_{j}$ and $p_{j}$. Where with Lagrange's equations it is sufficient to solve for only $q_{j}$. Given we know that the solutions are equivalent, projecting a solution $(q, p) \in \mathbb{R}^{2 n}$ in the phase plane to its first set of coordinates $q \in \mathbb{R}^{n}$ should coincide with those solutions in the configuration space.

The examples analogous to classical mechanics that we will consider are the Harmonic Oscillator, the Kepler problem and the relativistic Hamiltonian. They are divided into three separate cases, namely the one-dimensional, the two-dimensional and the three-dimensional problem. So that this chapter does not become too convoluted only the main results from each example will be collected here. The full calculations are provided in Appendix B for the one and two-dimensional cases, and in Chapter 6 for the three-dimensional case. We will find that to solve the problem for when $n=3$, we need the Hamilton-Jacobi theory which is covered at Chapters 5 and 6 . However, since we can still use the theory we have already to form the Hamilton function and its equations, as well as still carry out the corresponding analysis as we do with the other examples, we do include a discussion about the three-dimensional case here.

To obtain more explicit solutions the function $\psi \in C N\left(\mathbb{R}^{n}\right)$ will be taken to be

$$
\psi(p):=\frac{1}{\alpha}\|p\|^{\alpha}, \quad 1<\alpha \leq 2 .
$$

Recall that $\psi$ as above satisfies the conditions the Hamilton function needs, i.e. $\psi \in C N\left(\mathbb{R}^{n}\right) \cap C^{1}\left(\mathbb{R}^{n}\right)$ as well as being convex and coercive. Additionally we know from Example 3.2.4 that $\psi^{*} \in C^{2}\left(\mathbb{R}^{n}\right)$ hence the corresponding Lagrange's equations will be well-defined.

Following the solving of the differential equations are figures to enlighten us on the behaviour of the solutions we obtain. This will be especially useful as we will see it is not always possible to obtain explicit solutions. The one-dimensional examples come with phase diagrams for Hamilton's equations as they have solutions in a two-dimensional phase plane. These are construced using the computer programme Mathematica. Looking ahead to higher dimensions, we are no longer able to efficiently demonstrate the four-dimensional or six-dimensional phase diagrams when $n=2$ and $n=3$ respectively. Hence in these examples we use an alternative approach. We turn to another computer programme Matlab and use ODE solvers to obtain approximations for the solutions of Hamilton's equations. The ODE solvers use numerical methods to approximate solutions, hence the perturbation of accuracy due to the error cannot be ignored. To demonstrate this we include alongside each 
plot of the solutions a plot of the conserved value of the Hamilton function, E. As well as specifying the value of $E$ this plot has a secondary purpose in showing the efficiency of the numerical methods as $t$ changes. We know that for each inital condition $E$ has a corresponding constant value. However this may be misinterpreted after looking at the figures as $E$ will not appear constant. However a margin of error is a cost when employing numerical methods hence the changes we see in $E$ represent these errors playing a role. Larger changes indicate more error where less change indicates less error. One should look at the larger picture when reading the plots of $E$ and notice the range of $E$ only being of a small value. Hence the constant property of $E$ is strongly suggested being only slightly affected by the errors. We will see that these graphics are very beneficial in predicting what the solutions are actually doing. We leave the full details for the graphics themselves in each example.

\subsection{The Harmonic Oscillator}

In classical mechanics, the problem of the Harmonic Oscillator deals with particles that oscillate, or in other words have periodic motion. In one-dimension the potentials associated to such motion are usually of the form

$$
V(q)=c|q|^{2}
$$

where $c$ is some positive constant. Such an example can be found at [26, page 434].

We want to form an analogue to this problem with the Hamilton function. To do this we let the potentials be the Legendre transform of the kinetic term, i.e. $V(q):=\psi^{*}(q)$. Recollecting our chosen $\psi$ from (4.5) our Hamilton functions are therefore of the form

$$
\mathcal{H}(q, p)=\frac{1}{\alpha}\|p\|^{\alpha}+\frac{1}{\alpha^{*}}\|q\|^{\alpha^{*}} .
$$

We recollect from Example 1.17 that the Legendre transform of $\psi$ is $\frac{1}{\alpha^{*}}\|\cdot\|^{\alpha^{*}}, \alpha^{*}=$ $\frac{\alpha}{\alpha-1}$. Note that setting $\alpha=2$ in (4.1.2) we gain back a potential of the form of (4.1.1) and so a Hamiltonian representing the Harmonic Oscillator in classical mechanics. Hence (4.1.2) is a generalisation of sorts of what is studied in classical mechanics.

We recall from Theorem 3.3.1 that the Hamilton function has a conserved value $E$. Looking to Hamilton's equations as an initial value problem this constant $E$ may be determined through evaluating (4.1.2) at the initial conditions, i.e. $\mathcal{H}(q(t), p(t))=$ $\mathcal{H}\left(q_{0}, p_{0}\right)=E$. Given the structure of (4.1.2), we deduce that the conserved values of such Hamilton functions are always non-negative. This determination will prove to be important as we begin to look at each example below: 
Example 4.1.1. The Hamilton function whose dynamics we want to study is $\mathcal{H} \in$ $C^{1}\left(\mathbb{R}^{2}\right)$ such that

$$
\mathcal{H}(q, p)=\frac{1}{\alpha}|p|^{\alpha}+\frac{1}{\alpha^{*}}|q|^{\alpha^{*}},
$$

for $1<\alpha \leq 2$ and $\alpha^{*}=\frac{\alpha}{\alpha-1}$. Hamilton's equations follow as

$$
\left\{\begin{array}{l}
\dot{q}=p|p|^{\alpha-2} \\
\dot{p}=-q|q|^{\alpha^{*}-2}
\end{array}\right.
$$

We know the Hamilton function to be conserved determined by its initial values, i.e. $\mathcal{H}(q(t), p(t))=\mathcal{H}\left(q_{0}, p_{0}\right)=E$ for $q\left(t_{0}\right)=q_{0}$ and $p\left(t_{0}\right)=p_{0}$ fixed. Through this one can find $\dot{q}$ and $\dot{p}$ to satisfy:

$$
\left\{\begin{array}{l}
\dot{q}= \pm \alpha^{\frac{1}{\alpha^{*}}}\left(E-\frac{1}{\alpha^{*}}|q|^{\alpha^{*}}\right)^{\frac{1}{\alpha^{*}}} \\
\dot{p}= \pm \alpha^{* \frac{1}{\alpha}}\left(E-\frac{1}{\alpha}|p|^{\alpha}\right)^{\frac{1}{\alpha}}
\end{array}\right.
$$

The signs in the above are dependent on individual cases, i.e. when $q \geq 0$ and $p \geq 0$, $q \geq 0$ and $p<0, q<0$ and $p \geq 0$ and $q<0$ and $p<0$. We obtain different sign coefficients as the problem is carried out for each possible case. It was useful to solve the problem in this way due to the presence of absolute values at (4.1.4). The method is fully illustrated at Example B.1 of Appendix B. Through this $q(t)$ and $p(t)$ are solved to be

$$
q(t)=f_{1}^{-1}\left( \pm(\alpha E)^{\frac{1}{\alpha^{*}} t}+f_{1}\left(q_{0}\right)\right)
$$

and

$$
p(t)=f_{2}^{-1}\left( \pm\left(\alpha^{*} E\right)^{\frac{1}{\alpha}} t+f_{2}\left(p_{0}\right)\right) .
$$

The functions $f_{1}: M_{1} \longrightarrow M_{2}, M_{1}, M_{2}$ open in $\mathbb{R}$, and $f_{2}: N_{1} \longrightarrow N_{2}, N_{1}, N_{2}$ also open in $\mathbb{R}$, are defined as

$$
f_{1}(q):=q_{2} F_{1}\left(\frac{\alpha-1}{\alpha}, \frac{\alpha-1}{\alpha} ; \frac{2 \alpha-1}{\alpha} ; \frac{|q|^{\alpha^{*}}}{\alpha^{*} E}\right)
$$

and

$$
f_{2}(p):=p_{2} F_{1}\left(\frac{1}{\alpha}, \frac{1}{\alpha} ; \frac{\alpha+1}{\alpha} ; \frac{|p|^{\alpha}}{\alpha E}\right)
$$

respectively. The notation ${ }_{2} F_{1}$ is the hypergeometric series which is discussed in more detail at Appendix A. From there we find the solutions (4.1.6) and (4.1.7) to be well-defined whenever $|q|<\left(\alpha^{*} E\right)^{\frac{1}{\alpha^{*}}}$ and $|p|<(\alpha E)^{\frac{1}{\alpha}}$.

The corresponding Lagrange function is

$$
\mathcal{L}(q, \eta)=\frac{1}{\alpha^{*}}\left(|\eta|^{\alpha^{*}}-|q|^{\alpha^{*}}\right)
$$


with Lagrange's equation as:

$$
\ddot{q}|\dot{q}|^{\alpha^{*}-2}=-\frac{q|q|^{\alpha^{*}-2}}{\left(\alpha^{*}-1\right)} .
$$

Note for $q$ and $\eta$ fixed it holds that $\mathcal{L}(., \eta) \in C^{2}(\mathbb{R})$ and $\mathcal{L}(q,.) \in C^{2}(\mathbb{R})$.

From Theorem 3.3.2 we recollect that the conserved value $E$ of the Hamilton function may be given through the Lagrange function by

$$
\frac{\partial \mathcal{L}}{\partial \eta} \eta-\mathcal{L}=E
$$

Through this we can solve $\dot{q}$ to be:

$$
\dot{q}= \pm \alpha^{\frac{1}{\alpha^{*}}}\left(E-\frac{1}{\alpha^{*}}|q|^{\alpha^{*}}\right)^{\frac{1}{\alpha^{*}}}
$$

which coincides with the first equation of (4.1.5). Hence the solution for $q$ would follow as it did when solving Hamilton's equations. As before the sign coefficient at (4.1.11) is dependent on carrying out the problem for indivivdual cases, i.e. when $q \geq 0$ and $\dot{q} \geq 0, q \geq 0$ and $\dot{q}<0, q<0$ and $\dot{q} \geq 0$ and $q<0$ and $\dot{q}<0$. Once again the full details can be found at Example B.1.

Finally we note that we use the solution of $q$ with (4.1.11) to solve $\dot{q}$. The condition $|q|<\left(\alpha^{*} E\right)^{\frac{1}{\alpha^{*}}}$ confirms (4.1.11) to be well-defined.

For selected values of $\alpha$ below are some examples of the phase diagrams for (4.1.4) constructed using Mathematica. The horizontal axis represents the coordinate $q$ where the vertical axis corresponds to $p$. 


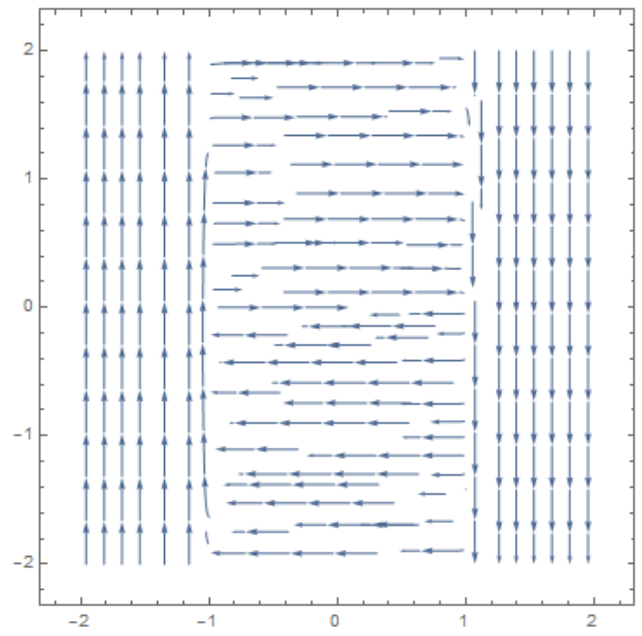

Figure 4.1.1: $\alpha=1.01$

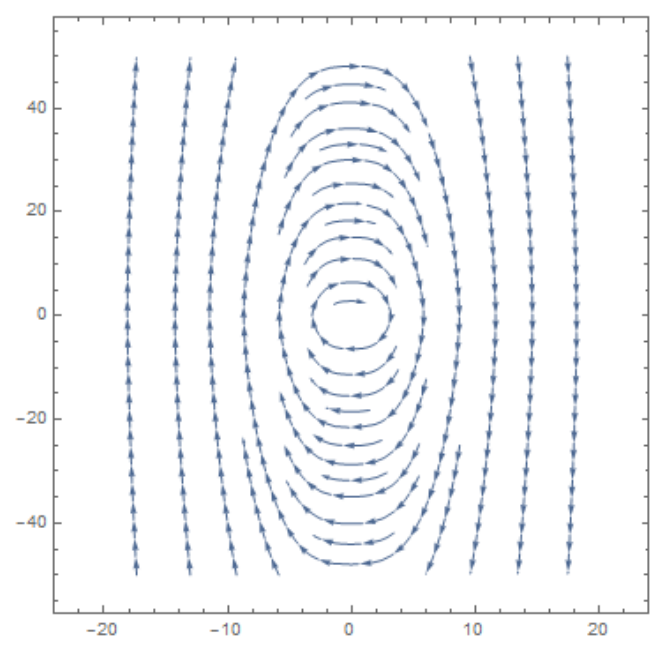

Figure 4.1.3: $\alpha=1.5$

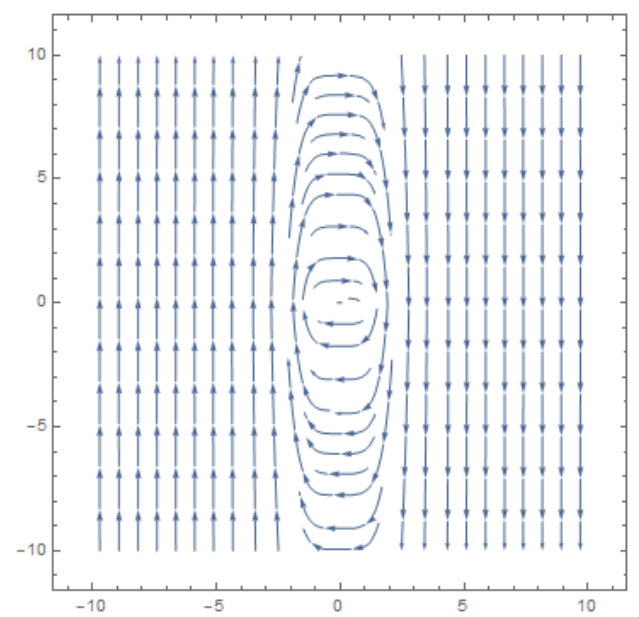

Figure 4.1.2: $\alpha=1.25$

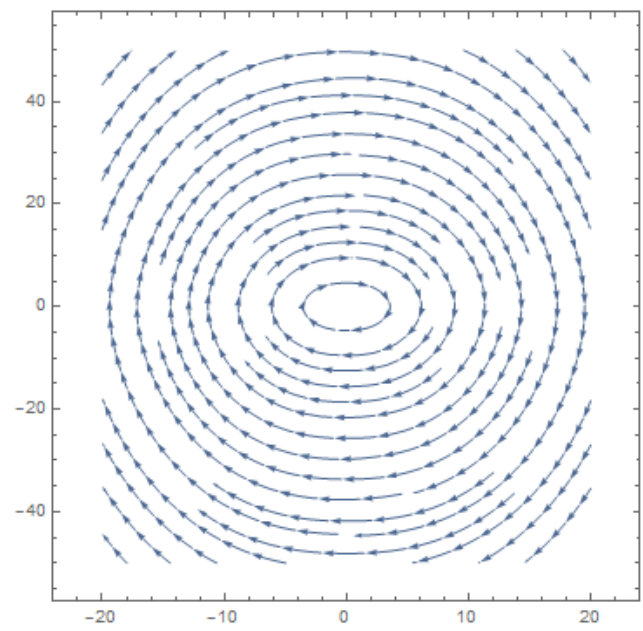

Figure 4.1.4: $\alpha=1.75$

Observing the above figures we can see that the solutions have a symmetric property when reflected over the lines $q=-p, p=0$ and $q=0$. In other words given the solutions for when $q \geq 0$ and $p \geq 0$, we can solve the cases for $q<0$ and $p<0$; $q \geq 0$ and $p<0 ; q<0$ and $p \geq 0$ by reflecting over $q=-p, p=0$ and $q=0$ respectively in the phase space and taking the appropriate sign. Additionally, they suggest that solutions are periodic. A possible explanantion are the hypergeomtric series included in $f_{1}$ and $f_{2}$ inducing a periodic behaviour.

As $\alpha$ tends to 2 , the above phase diagrams seem to converge to what one would expect from the solution of the Harmonic Oscillator in classical mechanics. This 
further emphasises the idea of this example acting as a generalisation of the case in classical mechanics. Taking $\alpha=2$ in Example 4.1.1, the Hamilton function and its Hamilton's equations would follow as

$$
\mathcal{H}(q, p)=\frac{1}{2} p^{2}+\frac{1}{2} q^{2}
$$

and

$$
\left\{\begin{array}{l}
\dot{q}=p, \\
\dot{p}=-q .
\end{array}\right.
$$

Following the calculations of Example 4.1.1 as seen in Example B.1 of Appendix B we would have for $q$ :

$$
\pm(2 E)^{-\frac{1}{2}} q_{2} F_{1}\left(\frac{1}{2}, \frac{1}{2} ; \frac{3}{2} ; \frac{q^{2}}{2 E}\right)=t+A_{1},
$$

$A_{1}$ a constant of integration. Recollecting (A.2) from Appendix A that

$$
\sin ^{-1} z=z_{2} F_{1}\left(\frac{1}{2}, \frac{1}{2} ; \frac{3}{2} ; z^{2}\right)
$$

equation (4.1.13) can be used to solve $q$ :

$$
q(t)=\sqrt{2 E} \sin \left( \pm t+\sin ^{-1}\left(\frac{q_{0}}{\sqrt{2 E}}\right)\right)
$$

where we have used $q(0)=q_{0}$. The second equation of (4.1.12) similarly gives:

$$
\pm(2 E)^{-\frac{1}{2}} p_{2} F_{1}\left(\frac{1}{2}, \frac{1}{2} ; \frac{3}{2} ; \frac{p^{2}}{2 E}\right)=t+A_{2},
$$

$A_{2}$ a further constant of integration. Using (4.1.14) once again and introducing the condition $p(0)=p_{0}$, we have

$$
p(t)=-\sqrt{2 E} \sin \left( \pm t-\sin ^{-1}\left(\frac{p_{0}}{\sqrt{2 E}}\right)\right) .
$$

Comparing to [26, Chapter 10.2] we see that these solutions do coincide well with the example for the Harmonic Oscillator in classical mechanics.

Example 4.1.2. The Hamilton function whose dynamics we want to study is $\mathcal{H} \in$ $C^{1}\left(\mathbb{R}^{4}\right)$ such that

$$
\mathcal{H}(q, p)=\frac{1}{\alpha}\|p\|^{\alpha}+\frac{1}{\alpha^{*}}\|q\|^{\alpha^{*}} .
$$


The associated Hamilton's equations follow as

$$
\left\{\begin{array}{l}
\dot{q}_{j}=p_{j}\|p\|^{\alpha-2}, \\
\dot{p}_{j}=-q_{j}\|q\|^{\alpha^{*}-2}, \quad j=1,2 .
\end{array}\right.
$$

Here we only illustrate the main steps for the above system. The full calculations can be found at Example B.3 at Appendix B.

Solving the above system can be difficult in its current form. Looking at the Hamilton function (4.1.16) it only involves the norms of $q$ and $p$. Therefore this Hamilton function has rotational symmetry. Hence, a good change of variables would be to polar coordinates, i.e. we use

$$
q_{1}=r \cos \vartheta \quad \text { and } \quad q_{2}=r \sin \vartheta
$$

This gives the new Hamilton function $\mathcal{H}_{2} \in C^{1}((0, \infty) \times[0,2 \pi) \times \mathbb{R} \backslash\{0\} \times \mathbb{R})$ such that

$$
\mathcal{H}_{2}\left(r, \vartheta, p_{r}, p_{\vartheta}\right)=\frac{1}{\alpha}\left(p_{r}^{2}+\frac{p_{\vartheta}^{2}}{r^{2}}\right)^{\frac{\alpha}{2}}+\frac{1}{\alpha^{*}} r^{\alpha^{*}} .
$$

Hamilton's equations now follow as:

$$
\begin{gathered}
\dot{r}=p_{r}\left(p_{r}^{2}+\frac{p_{\vartheta}^{2}}{r^{2}}\right)^{\frac{\alpha-2}{2}}, \\
\dot{\vartheta}=\frac{p_{\vartheta}}{r^{2}}\left(p_{r}^{2}+\frac{p_{\vartheta}^{2}}{r^{2}}\right)^{\frac{\alpha-2}{2}}, \\
\dot{p}_{r}=\frac{p_{\vartheta}^{2}}{r^{3}}\left(p_{r}^{2}+\frac{p_{\vartheta}^{2}}{r^{2}}\right)^{\frac{\alpha-2}{2}}-r^{\alpha^{*}-1},
\end{gathered}
$$

and

$$
\dot{p}_{\vartheta}=0
$$

implying that $p_{\vartheta}(t)=\kappa$ for all $t, \kappa$ being some constant. With $\vartheta$ a cyclic coordinate this agrees with the result of Theorem 3.3.5.

We treat the above system as an inital value problem. Hence we first try to find the general solutions. The constants will then be eliminated using the initial values $\left(r^{0}, \vartheta^{0}, p_{r}^{0}, p_{\vartheta}^{0}\right)$.

To begin we make use of Section 3.3 and establish any conserved quantities we can exploit. From Theorem 3.3.1 we know that the Hamilton function (4.1.18) has a conserved value $E$ determined by the initial values, i.e.

$$
\mathcal{H}_{2}\left(r(t), \vartheta(t), p_{r}(t), p_{\vartheta}(t)\right)=\mathcal{H}_{2}\left(r^{0}, \vartheta^{0}, p_{r}^{0}, p_{\vartheta}^{0}\right)=E>0 .
$$


Rearranging (4.1.22) and using $p_{\vartheta}=\kappa$ we find $p_{r}$ to be

$$
p_{r}= \pm \sqrt{\alpha^{\frac{2}{\alpha}}\left(E-\frac{1}{\alpha^{*}} r^{\alpha^{*}}\right)^{\frac{2}{\alpha}}-\frac{\kappa^{2}}{r^{2}}} .
$$

Through this the derivatives of the coordinates $r$ and $\vartheta$ can be found to satisfy

$$
\dot{r}= \pm \sqrt{\alpha^{\frac{2}{\alpha}}\left(E-\frac{1}{\alpha^{*}} r^{\alpha^{*}}\right)^{\frac{2}{\alpha}}-\frac{\kappa^{2}}{r^{2}}} \alpha^{\frac{\alpha-2}{\alpha}}\left(E-\frac{1}{\alpha^{*}} r^{\alpha^{*}}\right)^{\frac{\alpha-2}{\alpha}}
$$

and

$$
\dot{\vartheta}=\frac{\kappa \alpha^{\frac{\alpha-2}{\alpha}}}{r^{2}}\left(E-\frac{1}{\alpha^{*}} r^{\alpha^{*}}\right)^{\frac{\alpha-2}{\alpha}} .
$$

The coordinate $r$ is then determined by

$$
\gamma+t= \pm \int \frac{\alpha^{\frac{2-\alpha}{\alpha}} r\left(E-\frac{1}{\alpha^{*}} r^{\alpha^{*}}\right)^{\frac{2-\alpha}{\alpha}}}{\sqrt{\alpha^{\frac{2}{\alpha}} r^{2}\left(E-\frac{1}{\alpha^{*}} r^{\alpha^{*}}\right)^{\frac{2}{\alpha}}-\kappa^{2}}} \mathrm{~d} r
$$

for a constant of integration $\gamma$. When able to calculate the integral on the right-hand side of (4.1.26), we would obtain an expression in terms of $r$. Rearranging this new equation would then solve $r$ in terms of $t$ and the constants $E, \kappa$ and $\gamma$. This would give the general solution for $r$. The constant $\gamma$ may then be eliminated using the initial conditions and be found in terms of the initial values and $E$.

We may then substitute this into (4.1.25) to obtain a first-order differential equation involving only $\vartheta$. This equation can be used to find $\vartheta$ introducing a further constant of integration. This constant may then be eliminated as before using initial conditions. Having done this, we could then use our newly found solutions with (4.1.23) to solve for $p_{r}$.

The corresponding Lagrange function to (4.1.16) is

$$
\mathcal{L}(q, \eta)=\frac{1}{\alpha^{*}}\left(\|\eta\|^{\alpha^{*}}-\|q\|^{\alpha^{*}}\right)
$$

where $\mathcal{L}(., \eta) \in C^{2}\left(\mathbb{R}^{2}\right)$ and $\mathcal{L}(q,.) \in C^{2}\left(\mathbb{R}^{2}\right)$. Lagrange's equations appear as

$$
\ddot{q}_{j}\|\dot{q}\|^{\alpha^{*}-2}+\left(\alpha^{*}-2\right) \dot{q}_{j}\|\dot{q}\|^{\alpha^{*}-4} \sum_{l=1}^{2} \dot{q}_{l} \ddot{q}_{l}=-q_{j}\|q\|^{\alpha^{*-2}}, \quad j=1, \ldots, n,
$$

As with the Hamilton function it is useful to transform to polar coordinates. The Lagrange function can then be rewritten as a new function

$$
\mathcal{L}_{2}(r, \vartheta, \dot{r}, \dot{\vartheta})=\frac{1}{\alpha^{*}}\left(\left(\dot{r}^{2}+r^{2} \dot{\vartheta}^{2}\right)^{\frac{\alpha^{*}}{2}}-r^{\alpha^{*}}\right)
$$


$\mathcal{L}_{2}(., ., \dot{r}, \dot{\vartheta}) \in C^{1}((0, \infty) \times[0,2 \pi))$ and $\mathcal{L}_{2}(r, \vartheta, .,.) \in C^{2}\left(\mathbb{R}^{2}\right)$. Lagrange's equations follow as

$$
\frac{\mathrm{d}}{\mathrm{d} t}\left(\dot{r}\left(\dot{r}^{2}+r^{2} \dot{\vartheta}^{2}\right)^{\frac{\alpha^{*}-2}{2}}\right)=r \dot{\vartheta}^{2}\left(\dot{r}^{2}+r^{2} \dot{\vartheta}^{2}\right)^{\frac{\alpha^{*}-2}{2}}-r^{\alpha^{*}-1}
$$

and

$$
\frac{\mathrm{d}}{\mathrm{d} t}\left(r^{2} \dot{\vartheta}\left(\dot{r}^{2}+r^{2} \dot{\vartheta}^{2}\right)^{\frac{\alpha^{*}-2}{2}}\right)=0
$$

implying that $p_{\vartheta}(t)=\kappa$ for all $t, \kappa$ being some constant. Therefore the coordinate $p_{\vartheta}$ corresponding to the cyclic coordinate $\vartheta$ is conserved, as we expect from Theorem 3.3.6.

From Theorem 3.3.2 the conserved value E satisfies

$$
\frac{\partial \mathcal{L}_{2}}{\partial \dot{r}} \dot{r}+\frac{\partial \mathcal{L}_{2}}{\partial \dot{\vartheta}} \dot{\vartheta}-\mathcal{L}_{2}=E
$$

Through this the generalised velocities $\dot{r}$ and $\dot{\vartheta}$ can be found to be as they are at (4.1.24) and (4.1.25). Separation of variables then gives the solution (4.1.26) and so $r$ and $\vartheta$ may be deduced as they were before. Once having found $r$ and $\vartheta$, equations (4.1.24) and (4.1.25) may be used to find their derivatives.

Looking to the work so far there are some conditions we need to impose such that the results are well-defined:

(i) $E-\frac{1}{\alpha^{*}} r^{\alpha^{*}}>0$

(ii) $\alpha^{\frac{2}{\alpha}} r^{2}\left(E-\frac{1}{\alpha^{*}} r^{\alpha^{*}}\right)^{\frac{2}{\alpha}}-\kappa^{2}>0$.

Recall we have taken that $p_{r} \neq 0$. Then

$$
\frac{1}{\alpha}\left(p_{r}^{2}+\frac{p_{\vartheta}^{2}}{r^{2}}\right)^{\frac{\alpha}{2}}>0 .
$$

Taking $E$ as the Hamilton function at (4.1.18), and substituting into condition (i) above, equation (4.1.31) implies that the first condition is true.

Additionally $p_{r} \neq 0$ implies that

$$
p_{r}^{2} r^{2}>0
$$

Using (4.1.32) with (4.1.31) and taking $E$ as the Hamilton function as before, we find the second condition above to also be true. 
As was forewarned in the introduction of this chapter, we have a solution (4.1.26) with a form that does not tell us much about its behaviour. Hence with the help of Matlab below are figures presenting plots approximating the evolution of the solutions of Hamilton's equations and the value E. In each case it is specified in the captions the specific value of $\alpha$ and the corresponding initial values that were taken. 

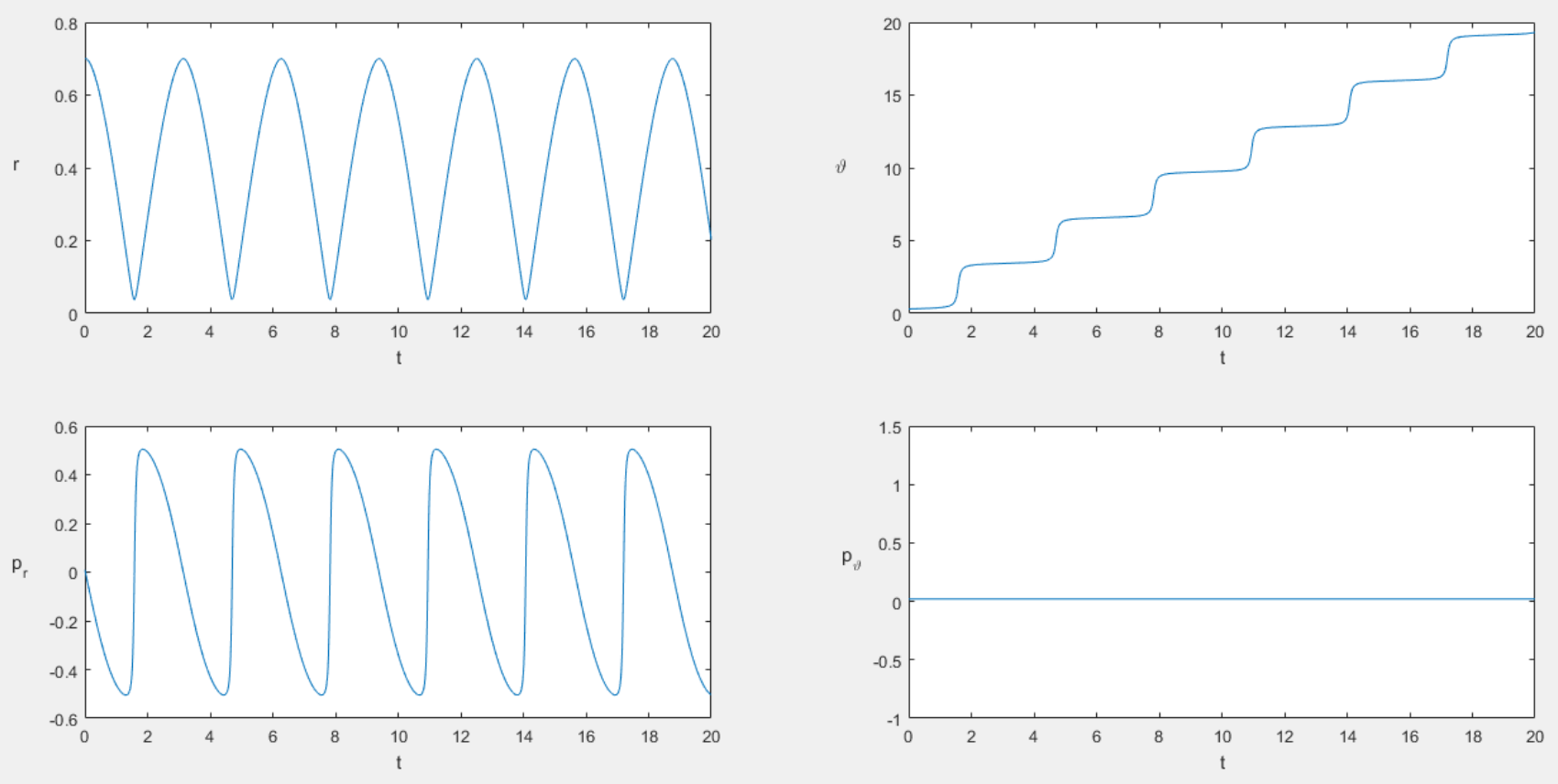

Figure 4.1.5: $\alpha=1.75, \quad \mathcal{H}_{2}(0.7,0.3,0.01,0.02)=0.187715$

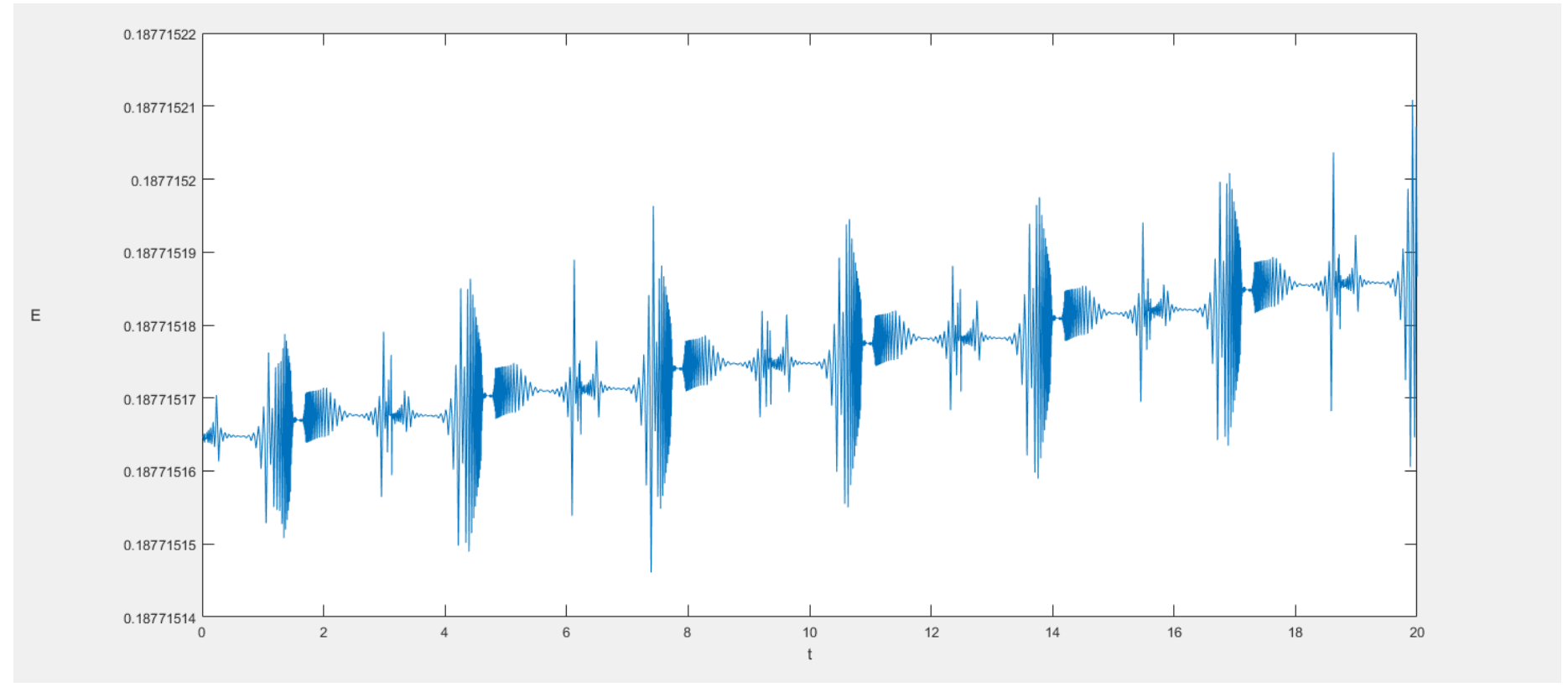

Figure 4.1.6 

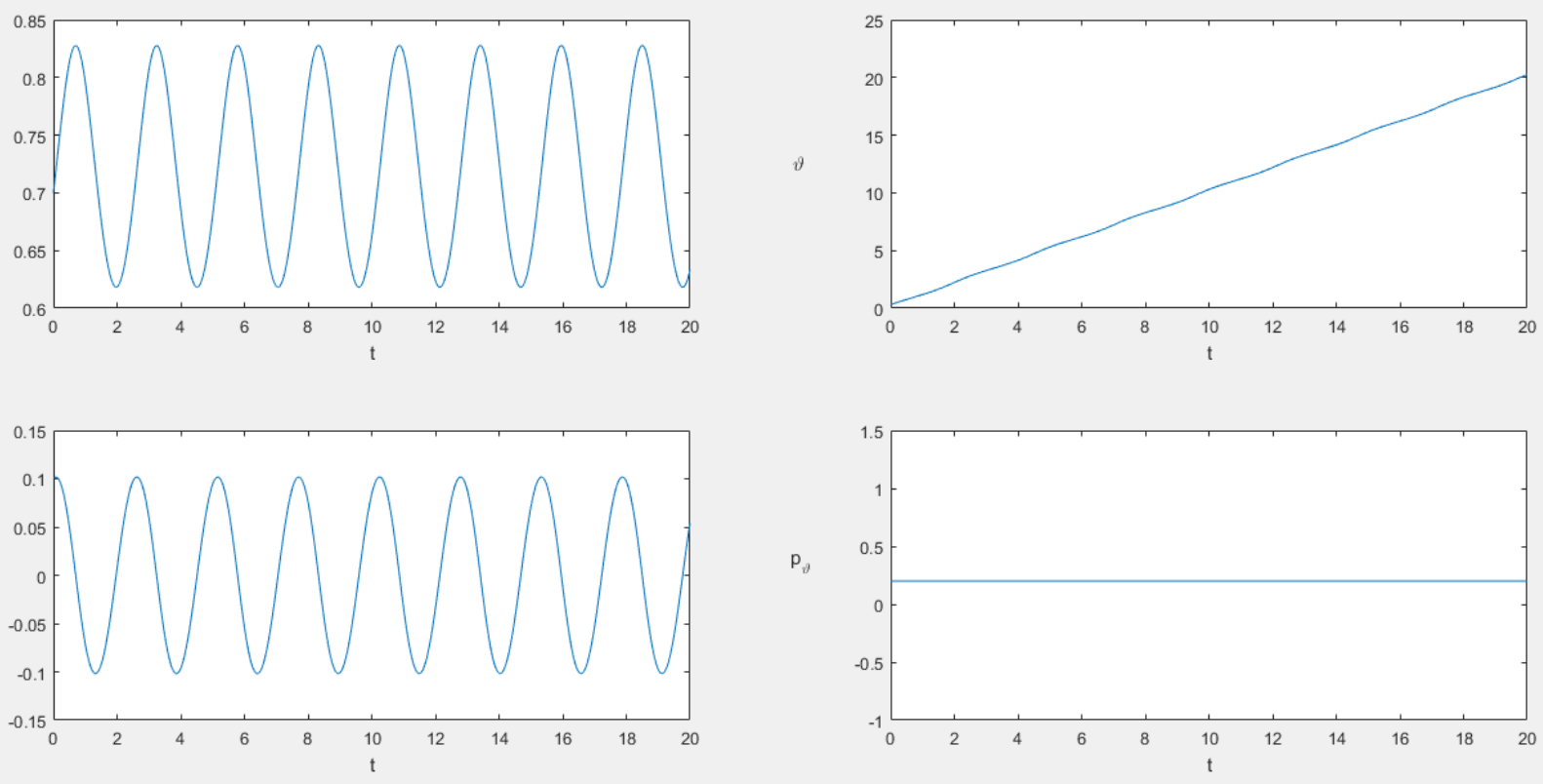

Figure 4.1.7: $\alpha=1.25, \quad \mathcal{H}_{2}(0.7,0.3,0.1,0.2)=0.2132409$

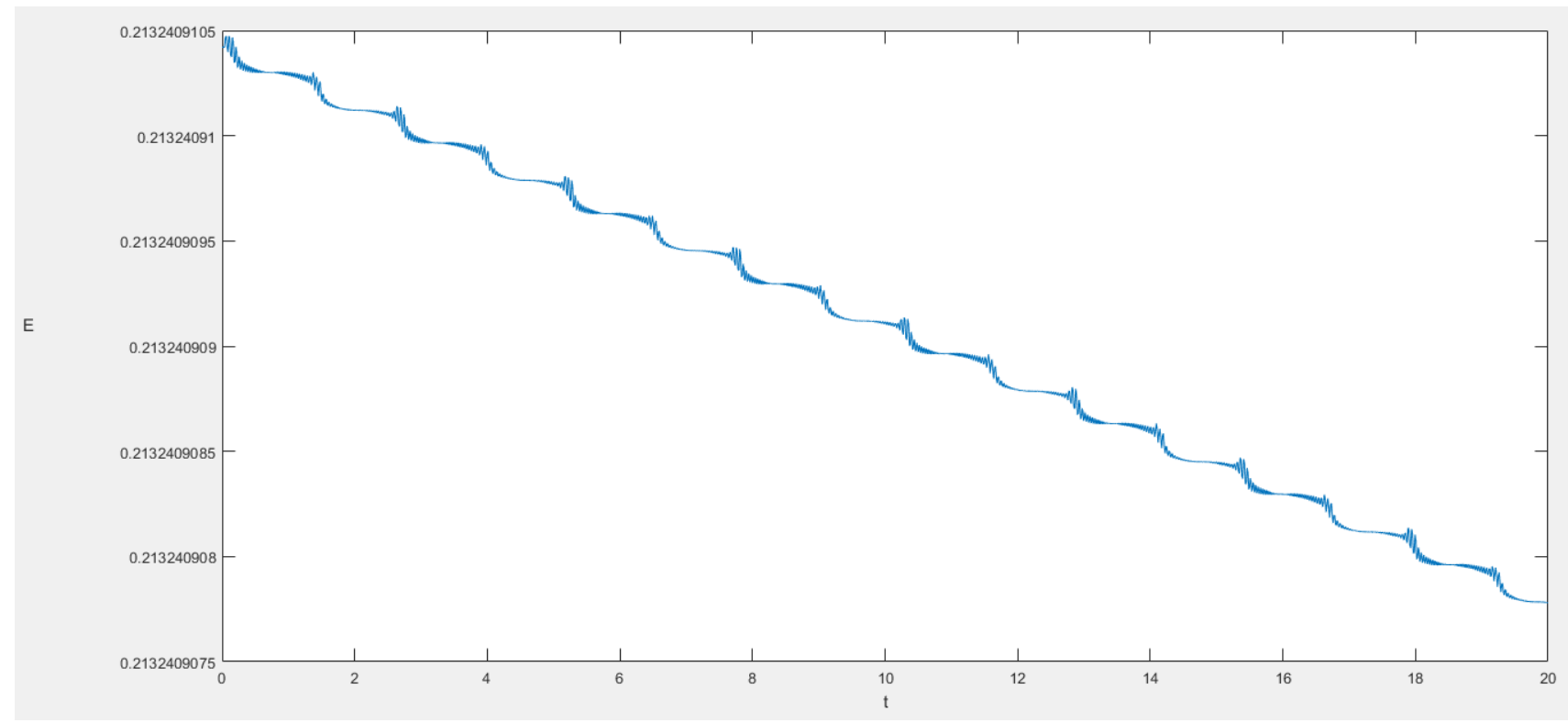

Figure 4.1.8 

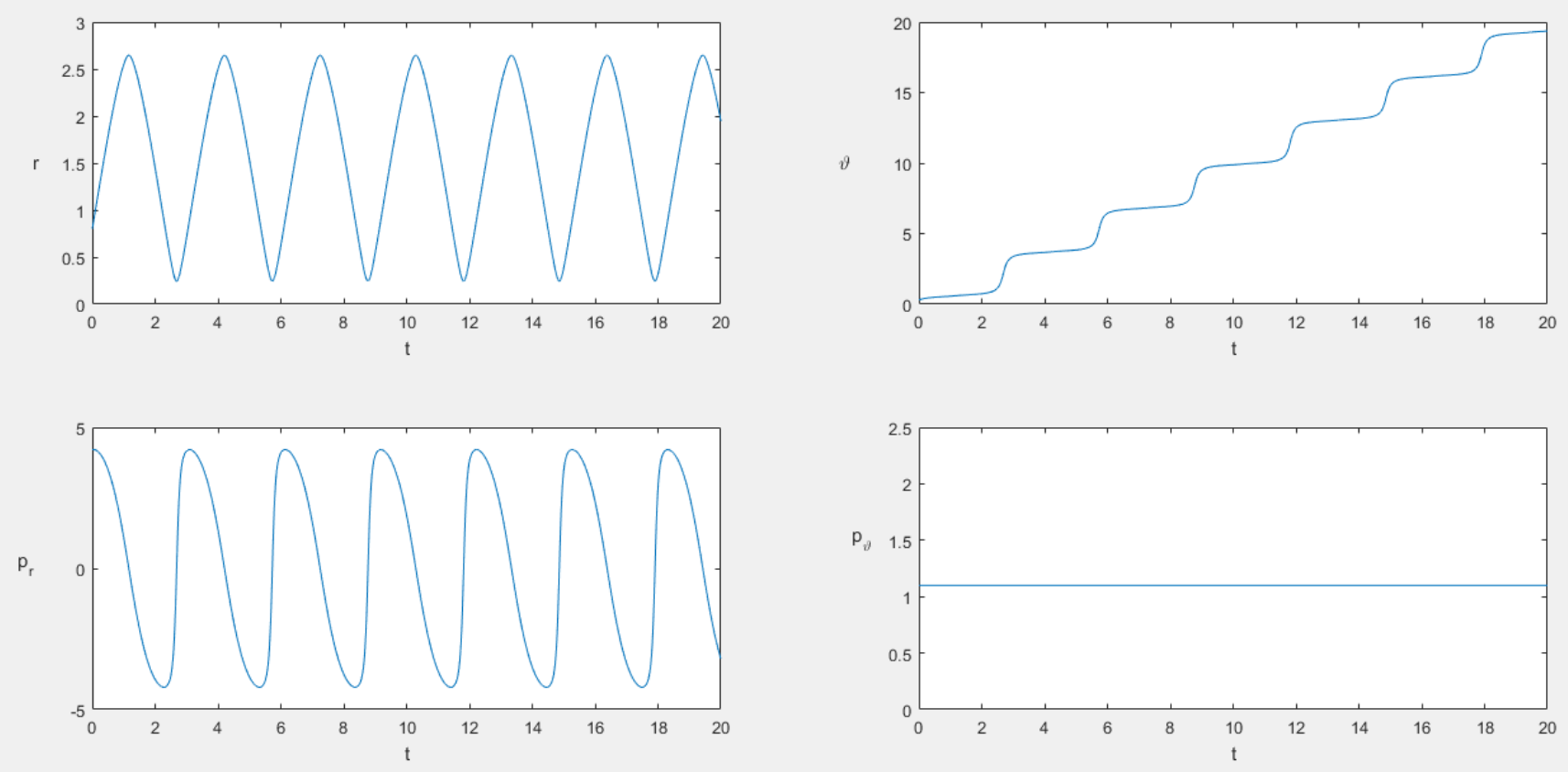

Figure 4.1.9: $\alpha=1.5, \quad \mathcal{H}_{2}(0.8,0.3,4.2,1.1)=6.3643$

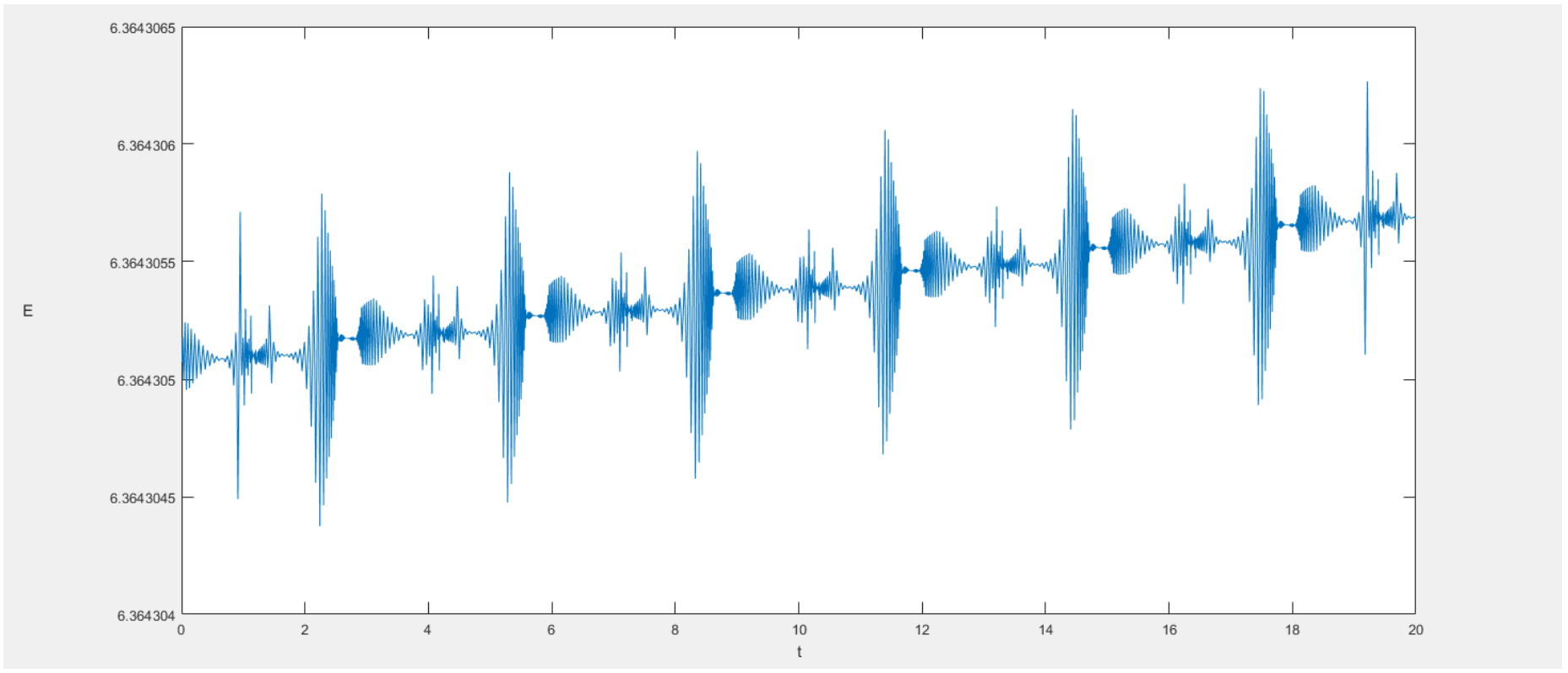

Figure 4.1.10 

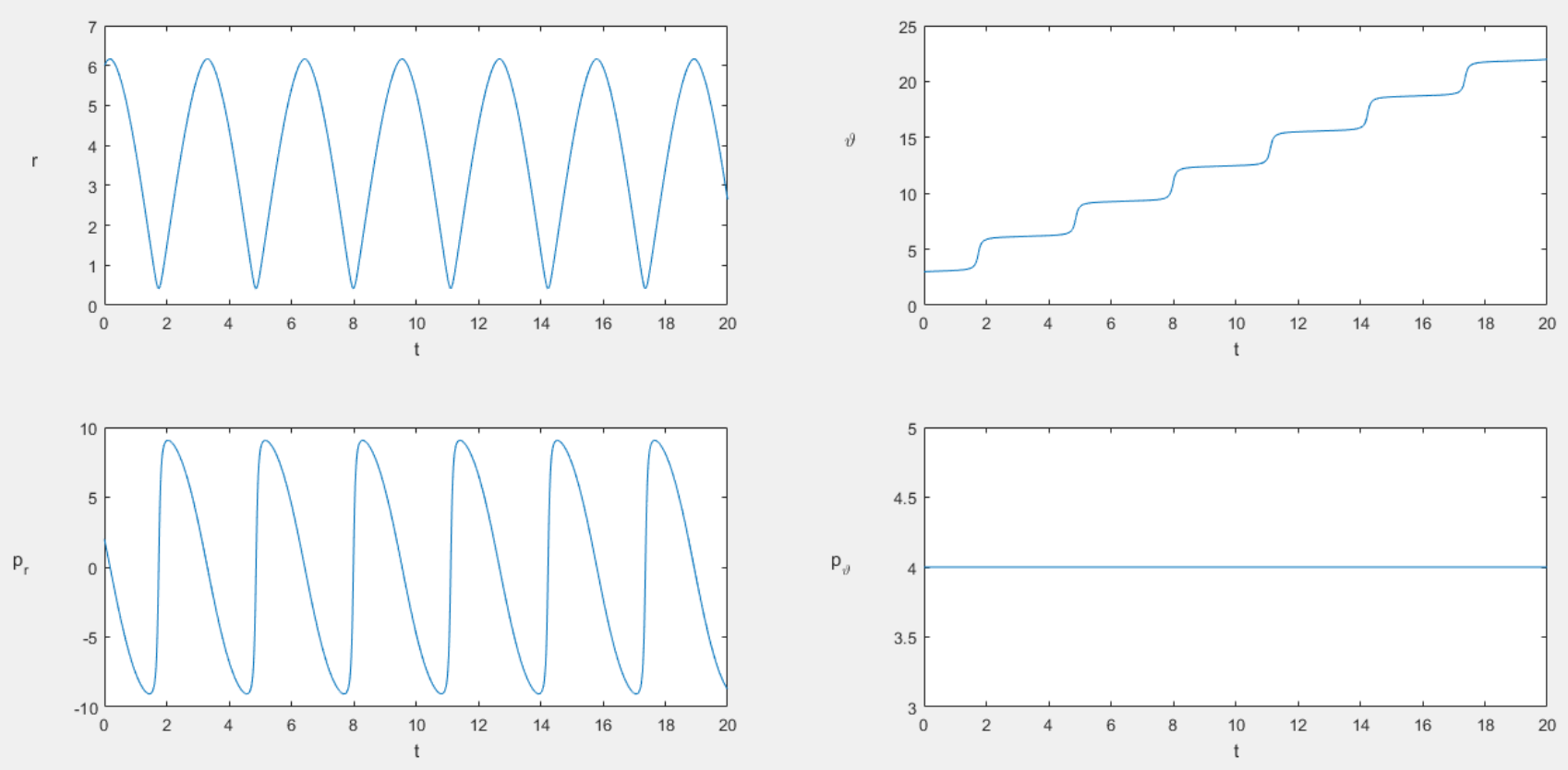

Figure 4.1.11: $\alpha=1.75, \quad \mathcal{H}_{2}(6,3,2,4)=30.1432$

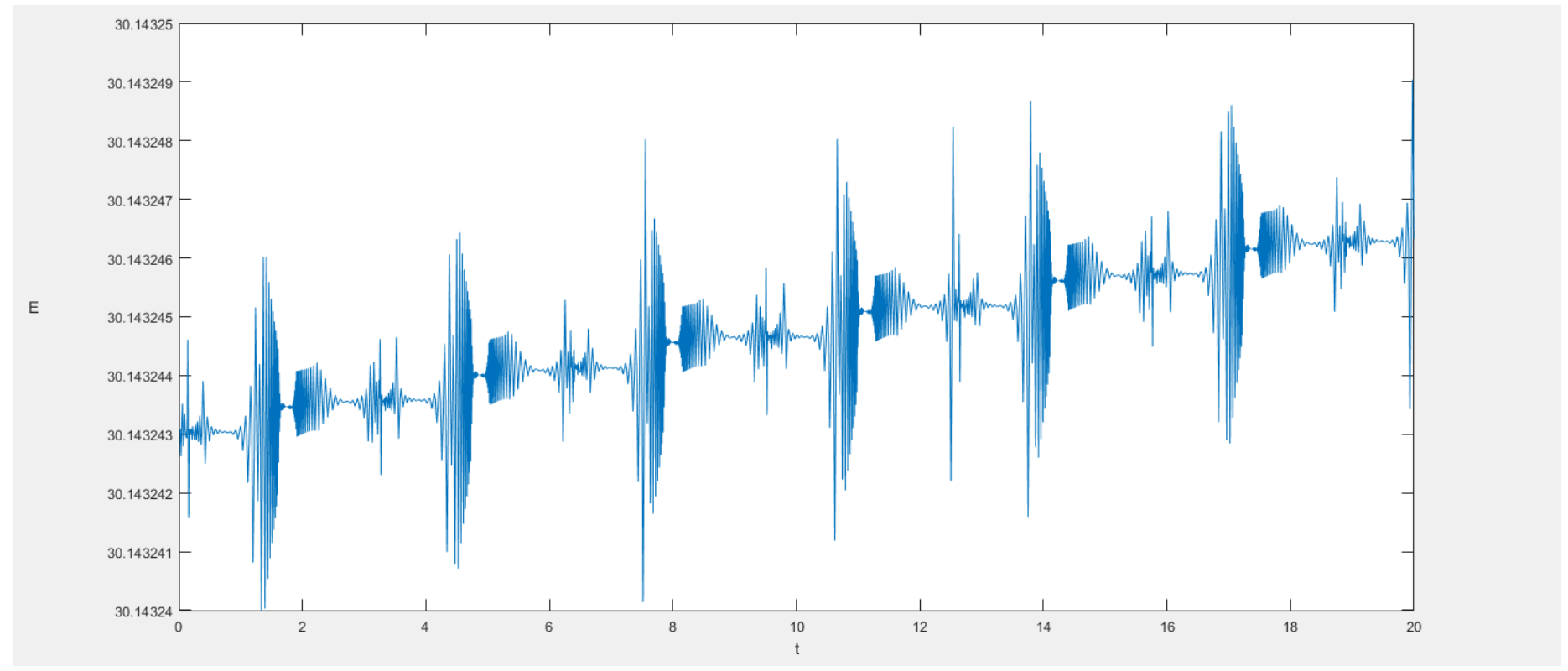

Figure 4.1 .12 

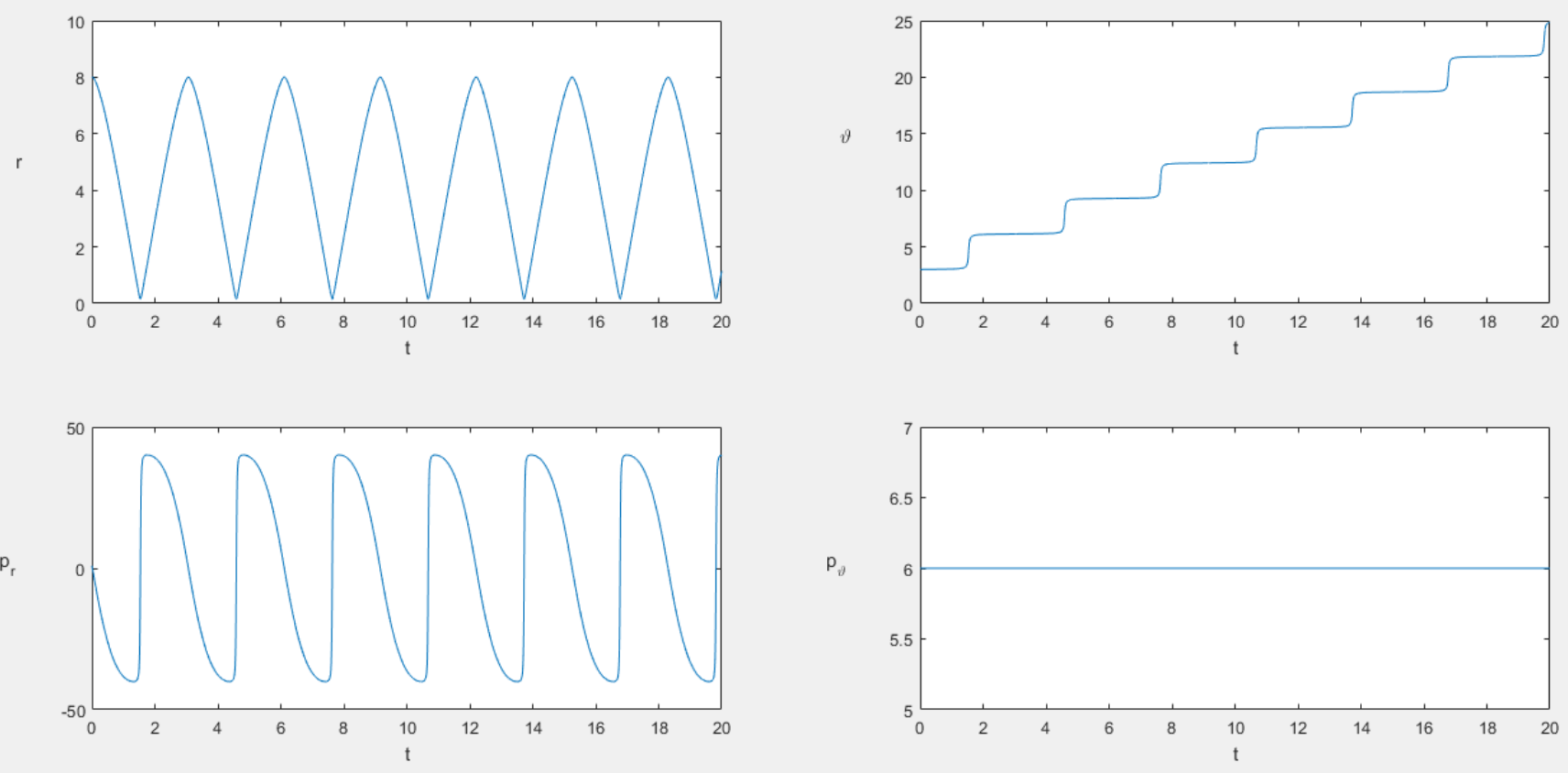

Figure 4.1.13: $\alpha=1.5, \quad \mathcal{H}_{2}(8,3,1,6)=171.598$

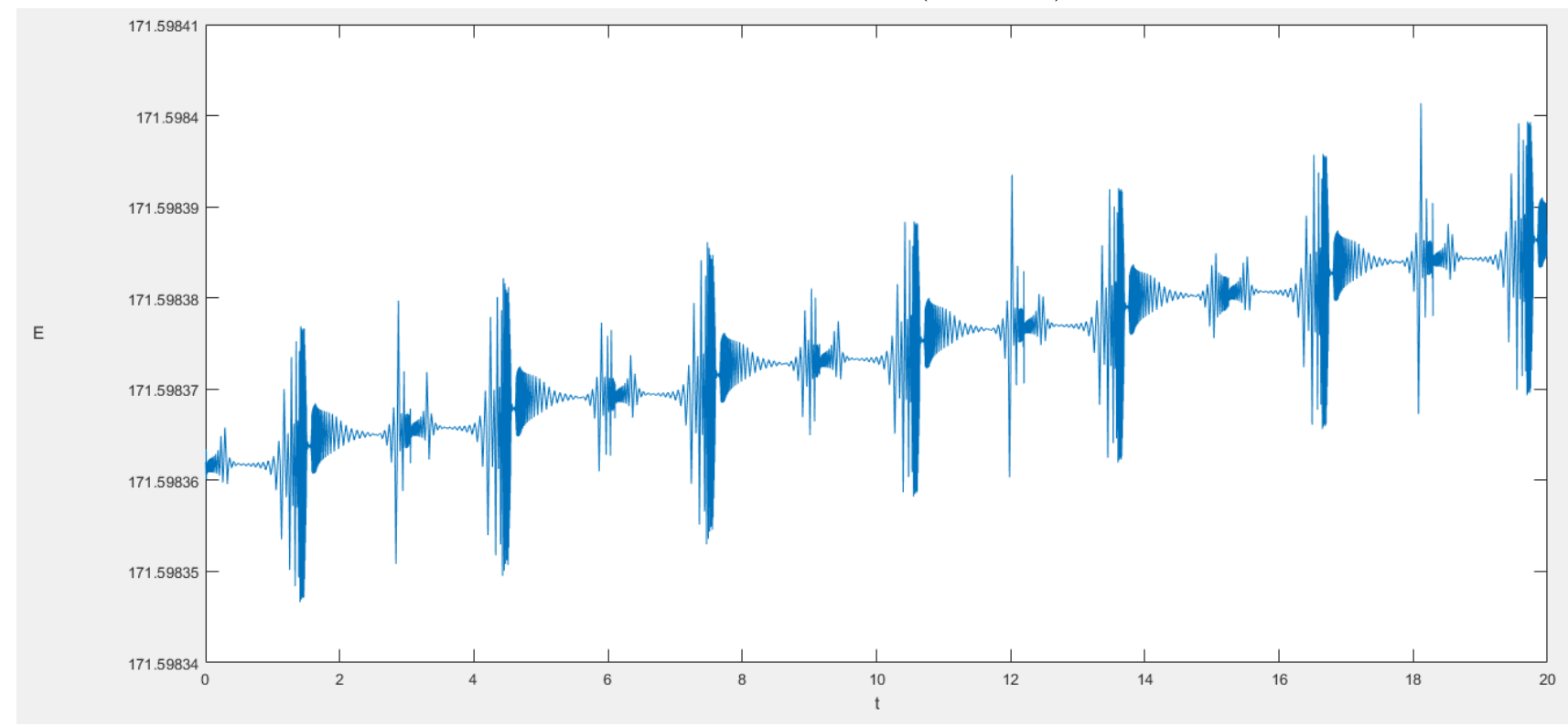

Figure 4.1.14 
It is clear from the preceeding figures that periodic behaviour is strongly suggested. To further support this conclusion we discuss now in more detail what we deduce about each coordinate from these figures.

To begin with the simplest behaviour we look to the bottom right graph of each figure. Here the coordinate $p_{\vartheta}$ is constant every time. This is unsurprising given we have already deduced this from the differential equation (4.1.21).

Looking to the graph of $r$ in the top left corners, we see that $r$ oscillates between a maximum and minimum value with its periodicity being largely the same as $E$ increases. Overall the range of these values seems to increase as $E \rightarrow \infty$. This is clear oscillatory behaviour as one would expect with an example for the Harmonic Oscillator in classical mechanics. The corresponding coordinate $p_{r}$ unsurprisingly also suggests the same behaviour oscillating between values. It seems to have a similar periodicity to $r$ with its graph being slightly translated when compared to $r$. Additionally it undergoes large change whenever $r$ has little change and vice versa. From a more physical point of view in mechanics, this signifies the large change in momentum as the particle slows down or accelerates while also showing the more steady momentum when the particle travels more consistently. Overall $p_{r}$ seems to also increase as $E$ increases, possibly due to the larger radi that are in play at this point. Again thinking in terms of mechanics there is more distance to accelerate through.

Let us look at $\vartheta$. Overall the coordinate seems to increase at a steady rate, almost one-to-one with the inrcease in $t$. However a closer look indicates small fluctuations occurring at ends of consistent intervals of $t$. Looking across to the graph of $r$ this seems to be in line with whenever the radius attains its minimum value. Comparing to the figures of higher or lower values for E, for example Figures 4.1.5 and 4.1.13, the fluctuations are much larger. Additionally the maximum values of $\vartheta$ seems to increase with E also.

We can collect all of the behaviours observed so far and describe what kind of path would be illustrated in in the two-dimensional plane mapping $r$ against $\vartheta$. Overall, the shape of a flower with petals seems to be traced. As $\vartheta$ increases this flower path is repeated circling the origin. However at lower and higher end energies the petals seem to be constrained along less changes in $\vartheta$, therefore being thinner. Where in Figure 4.1.7, the petals are much wider with $\vartheta$ having less constrained values. With $r, \vartheta$ and $p_{r}$ all seeming to increase as $E$ gets larger, this suggests that the path given by the petals gets larger and rotates quicker within the plane.

To include $E$ into the discussion we note that these figures have values all close to each other mapped against $t$. This is unsurprising given we know $E$ to be the conserved value of the Hamilton function. As mentioned before the small change is caused by the error in the numerical method used to generate these results. However this shows how good the approximation actually is given how small in size the range 
in values of $E$ are.

Similar to $\vartheta$ the figures of $E$ demonstrate periodic fluctuations. Comparing to $r$ and $p_{r}$ each fluctuations occurs whenever $r$ reaches its maximum or minimum value, or in other words whenevrer $p_{r}$ is at its greatest change. This shows the error being largely effected whenever a large change occurs in the behaviour. The opposite happens when the change is more gradual.

Example 4.1.3. The Hamilton function whose dynamics we want to study is $\mathcal{H} \in$ $C^{1}\left(\mathbb{R}^{6}\right)$ such that

$$
\mathcal{H}(q, p)=\frac{1}{\alpha}\|p\|^{\alpha}+\frac{1}{\alpha^{*}}\|q\|^{\alpha^{*}}
$$

Hamilton's equations follow as

$$
\left\{\begin{array}{l}
\dot{q}_{j}=p_{j}\|p\|^{\alpha-2}, \\
\dot{p}_{j}=-q_{j}\|q\|^{\alpha^{*}-2},
\end{array} \quad j=1,2,3 .\right.
$$

As in the two-dimensional example, the above system can be difficult to solve in its current state. But similar to the two-dimensional case the Hamilton function only involves the norms of the relevant coordinates, therefore we again have a rotational symmetry. Hence a good change of variables would be to the three-dimensional analogue to polar coordinates, namely spherical polar coordinates, i.e. we use

$$
q_{1}=r \sin \phi \cos \theta, \quad q_{2}=r \sin \phi \sin \theta, \quad \text { and } \quad q_{3}=r \cos \phi .
$$

The function (4.1.33) is then equivalent to $\mathcal{H}_{3} \in C^{1}((0, \infty) \times[0, \pi] \times[0,2 \pi) \times \mathbb{R} \backslash$ $\left.\{0\} \times \mathbb{R}^{2}\right)$ such that

$$
\mathcal{H}_{3}\left(r, \phi, \theta, p_{r}, p_{\phi}, p_{\theta}\right)=\frac{1}{\alpha}\left(p_{r}^{2}+\frac{p_{\phi}^{2}}{r^{2}}+\frac{p_{\theta}^{2}}{r^{2} \sin ^{2} \phi}\right)^{\frac{\alpha}{2}}+\frac{1}{\alpha^{*}} r^{\alpha^{*}} .
$$

Hamilton's equations follow as

$$
\begin{gathered}
\dot{r}=p_{r}\left(p_{r}^{2}+\frac{p_{\phi}^{2}}{r^{2}}+\frac{p_{\theta}^{2}}{r^{2} \sin ^{2} \phi}\right)^{\frac{\alpha-2}{2}}, \\
\dot{\phi}=\frac{p_{\phi}}{r^{2}}\left(p_{r}^{2}+\frac{p_{\phi}^{2}}{r^{2}}+\frac{p_{\theta}^{2}}{r^{2} \sin ^{2} \phi}\right)^{\frac{\alpha-2}{2}}, \\
\dot{\theta}=\frac{p_{\theta}}{r^{2} \sin ^{2} \phi}\left(p_{r}^{2}+\frac{p_{\phi}^{2}}{r^{2}}+\frac{p_{\theta}^{2}}{r^{2} \sin ^{2} \phi}\right)^{\frac{\alpha-2}{2}}, \\
\dot{p}_{r}=\frac{1}{r^{3}}\left(p_{\phi}^{2}+\frac{p_{\theta}^{2}}{\sin ^{2} \phi}\right)\left(p_{r}^{2}+\frac{p_{\phi}^{2}}{r^{2}}+\frac{p_{\theta}^{2}}{r^{2} \sin ^{2} \phi}\right)^{\frac{\alpha-2}{2}}-r^{\alpha^{*}-1},
\end{gathered}
$$




$$
\dot{p}_{\phi}=\frac{p_{\theta}^{2} \cos \phi}{r^{2} \sin ^{3} \phi}\left(p_{r}^{2}+\frac{p_{\phi}^{2}}{r^{2}}+\frac{p_{\theta}^{2}}{r^{2} \sin ^{2} \phi}\right)^{\frac{\alpha-2}{2}},
$$

and

$$
\dot{p}_{\theta}=0,
$$

implying that $p_{\theta}(t)=\beta_{3}$ for all $t, \beta_{3}$ being some constant. Therefore the coordinate $p_{\theta}$ corresponding to the cyclic coordinate $\theta$ is conserved as expected from Theorem 3.3.5.

The Hamilton function $\mathcal{H}_{3}$ may be considered as an equivalent function of only four variables. We choose to denote this by $\tilde{\mathcal{H}}_{3} \in C^{1}((0, \infty) \times[0,2 \pi) \times \mathbb{R} \backslash\{0\} \times \mathbb{R})$

$$
\tilde{\mathcal{H}}_{3}\left(r, \theta, p_{r}, p_{\theta}\right)=\frac{1}{\alpha}\left(p_{r}^{2}+\frac{\beta_{2}^{2}}{r^{2}}\right)^{\frac{\alpha}{2}}+\frac{1}{\alpha^{*}} r^{\alpha^{*}}
$$

where

$$
\beta_{2}^{2}=p_{\phi}^{2}+\frac{\beta_{3}^{2}}{\sin ^{2} \phi} .
$$

The above function (4.1.41) coincides with the Hamilton function (4.1.18), the two-dimensional case analogous to this example. In this comparison we see that $\beta_{2}$ must be the conserved coordinate $p_{\vartheta}$ found in Example 4.1.2.

To solve the above system of equations we need to turn to the theory of HamiltonJacobi which is covered later in the thesis in Chapters 5 and 6 . Hence in this example we simply list the solutions we would obtain and carry out the corresponding analysis as we have done with previous examples. To see the full details of how these solutions are obtained, we refer to Example 6.1.3 of Chapter 6.

By the theory of Hamilton-Jacobi it can be shown that $\beta_{2}$ is constant such that

$$
r^{2}\left(\alpha^{\frac{2}{\alpha}}\left(E-\frac{1}{\alpha^{*}} r^{\alpha^{*}}\right)^{\frac{2}{\alpha}}-p_{r}^{2}\right)=p_{\phi}^{2}+\frac{\beta_{3}^{2}}{\sin ^{2} \phi}=\beta_{2}^{2} .
$$

Through this the solutions are found to be

$$
\begin{gathered}
p_{r}= \pm \sqrt{\alpha^{\frac{2}{\alpha}}\left(E-\frac{1}{\alpha^{*}} r^{\alpha^{*}}\right)^{\frac{2}{\alpha}}-\frac{\beta_{2}^{2}}{r^{2}}}, \\
p_{\phi}= \pm \sqrt{\beta_{2}^{2}-\frac{\beta_{3}^{2}}{\sin ^{2} \phi}}, \\
p_{\theta}=\beta_{3}, \\
\gamma_{1}= \pm \int \frac{\alpha^{\frac{2-\alpha}{\alpha}} r\left(E-\frac{1}{\alpha^{*}} r^{\left.\alpha^{*}\right)^{\frac{2-\alpha}{\alpha}}}\right.}{\sqrt{\alpha^{\frac{2}{\alpha}} r^{2}\left(E-\frac{1}{\alpha^{*}} r^{\alpha^{*}}\right)^{\frac{2}{\alpha}}-\beta_{2}^{2}}} \mathrm{~d} r
\end{gathered}
$$




$$
\gamma_{2} \pm \int \frac{\beta_{2}}{r \sqrt{\alpha^{\frac{2}{\alpha}} r^{2}\left(E-\frac{1}{\alpha^{*}} r^{\alpha^{*}}\right)^{\frac{2}{\alpha}}-\beta_{2}^{2}}} \mathrm{~d} r= \pm \int \frac{\beta_{2} \sin \phi}{\sqrt{\beta_{2}^{2} \sin ^{2} \phi-\beta_{3}^{2}}} \mathrm{~d} \phi
$$

and

$$
\gamma_{3} \pm \int \frac{\beta_{3}}{\sin \phi \sqrt{\beta_{2}^{2} \sin ^{2} \phi-\beta_{3}^{2}}} \mathrm{~d} \phi=\theta
$$

for constants of integration $\gamma_{i}, i=1,2,3$. Through integrating (if possible) the righthand side of (4.1.45), an expression for $r$ in terms of $t$ can be obtained. The constant of integration may then be eliminated through initial conditions as before.

The solution for $r$ may then be substituted into (4.1.46) which can then be solved similarly for $\phi$. As before any constant may be eliminated through initial conditions. This process may be similarly repeated a third time with (4.1.47) to solve for $\theta$.

As with the two-dimensional case, there are some conditions we must check so that the results are well-defined:

(i) $E-\frac{1}{\alpha^{*}} r^{\alpha^{*}}>0$

(ii) $\alpha^{\frac{2}{\alpha}} r^{2}\left(E-\frac{1}{\alpha^{*}} r^{\alpha^{*}}\right)^{\frac{2}{\alpha}}-\beta_{2}^{2}>0$,

(iii) $\beta_{2}^{2}-\frac{\beta_{3}^{2}}{\sin ^{2} \phi}>0$.

We follow a similar apprach to what was seen in the two-dimensional case at Example 4.1.2. Recall that $p_{r} \neq 0$. Then

$$
\frac{1}{\alpha}\left(p_{r}^{2}+\frac{p_{\phi}^{2}}{r^{2}}+\frac{p_{\theta}^{2}}{r^{2} \sin ^{2} \phi}\right)^{\frac{\alpha}{2}}>0 .
$$

Taking $E$ as the Hamilton function at (4.1.35) and substituting this into condition (i), the inequality at (4.1.48) then implies that the first condition above is true.

For the second condition we note that $p_{r} \neq 0$ of course implies that $p_{r}^{2}>0$. Recollecting equation (4.1.42) we have

$$
r^{2}\left(\alpha^{\frac{2}{\alpha}}\left(E-\frac{1}{\alpha^{*}} r^{\alpha^{*}}\right)^{\frac{2}{\alpha}}-p_{r}^{2}\right)=\beta_{2}^{2}
$$

Rearranging and using $p_{r}^{2}>0$ we obtain

$$
\alpha^{\frac{2}{\alpha}}\left(E-\frac{1}{\alpha^{*}} r^{\alpha^{*}}\right)^{\frac{2}{\alpha}}-\frac{\beta_{2}^{2}}{r^{2}}=p_{r}^{2}>0,
$$

i.e. the second condition. 
Finally we also know from equation (4.1.42) that

$$
p_{\phi}^{2}+\frac{\beta_{3}^{2}}{\sin ^{2} \phi}=\beta_{2}^{2} .
$$

This implies that $\beta_{3}<\beta_{2}$ must hold. This estimate then implies that the condition (iii) must also be true.

As was discussed in the introduction of this chapter, we have solutions of forms that do not tell us much about their behaviour. Hence with the help of Matlab, below are figures presenting plots approximating the evolution of the solutions of Hamilton's equations and the value E. In each case it is specified in the captions the specific value of $\alpha$ as well as the corresponding initial values that were taken. 

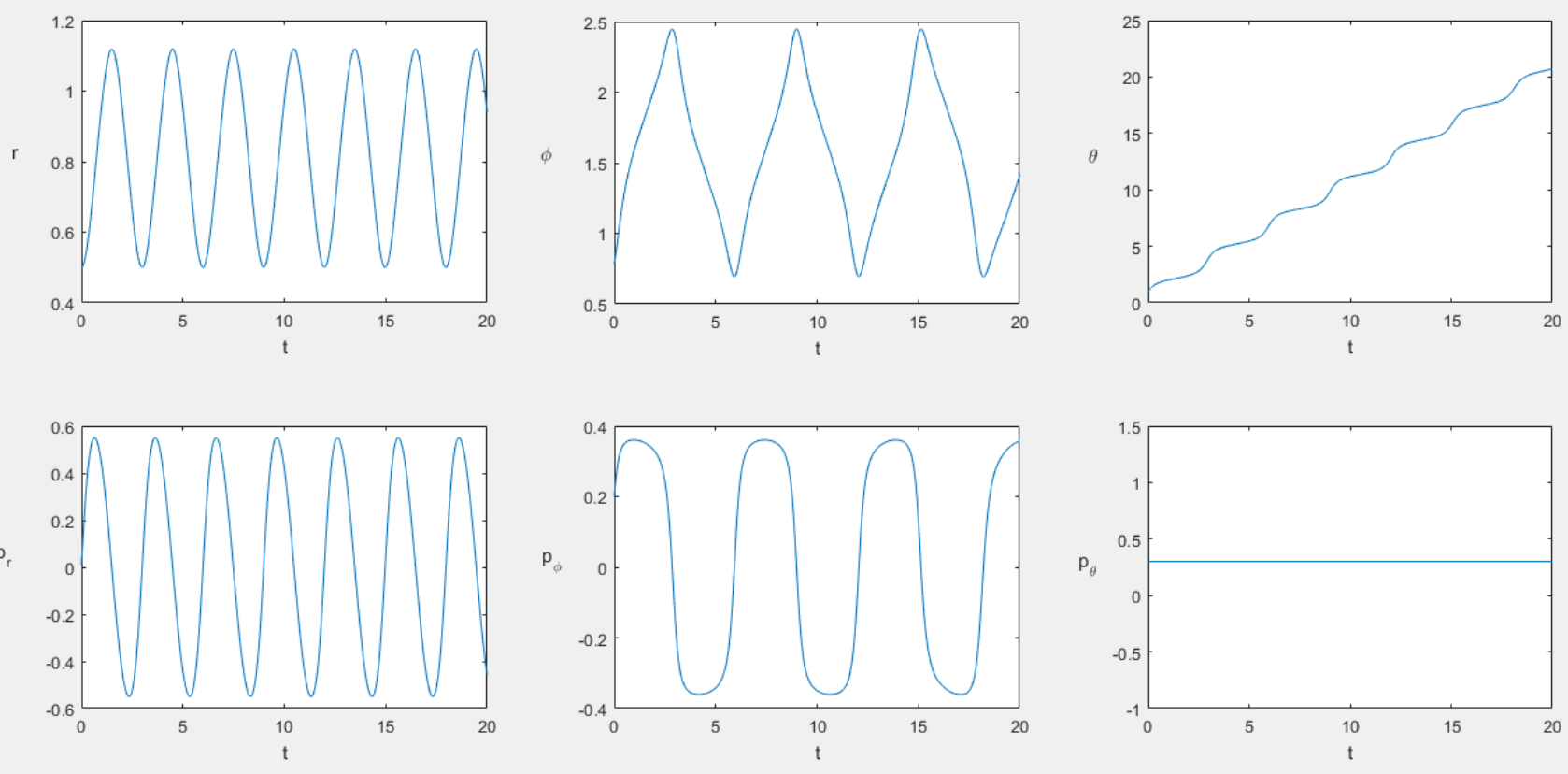

Figure 4.1.15: $\alpha=1.5, \quad \mathcal{H}_{3}\left(0.5, \frac{\pi}{4}, 1,0.01,0.2,0.3\right)=0.6474366$

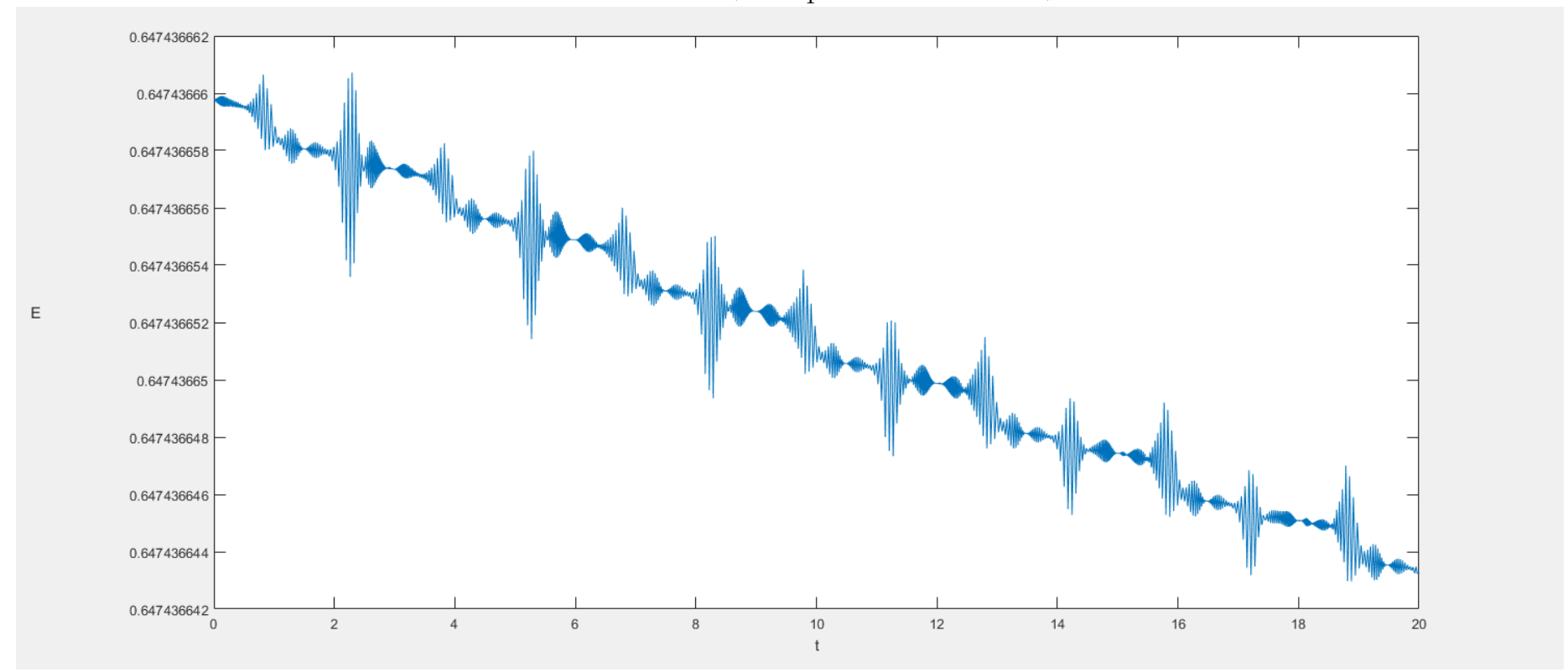

Figure 4.1.16 

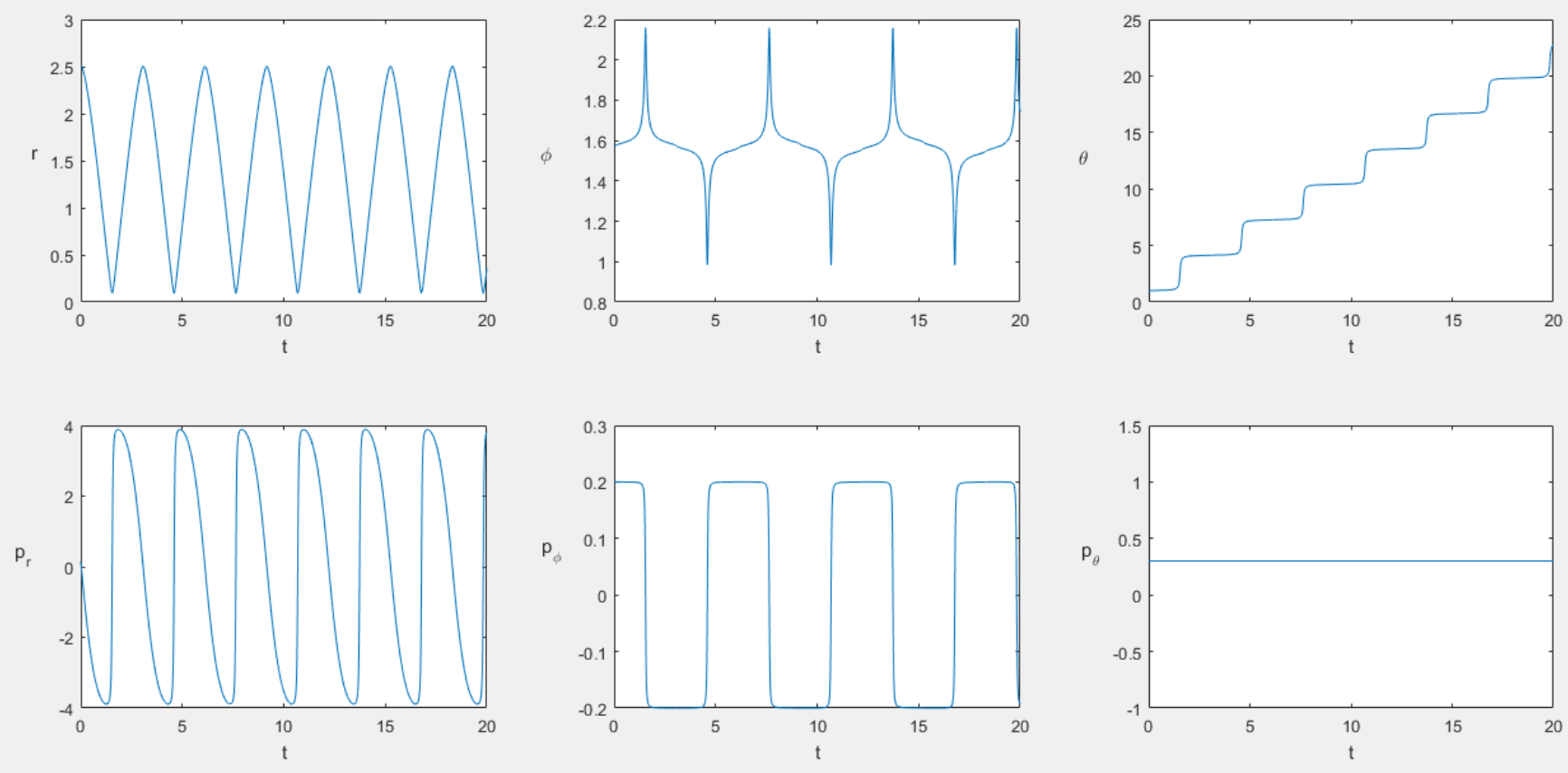

Figure 4.1.17: $\alpha=1.5, \quad \mathcal{H}_{3}\left(2.5, \frac{\pi}{4}, 1,0.15,0.2,0.3\right)=5.27161$

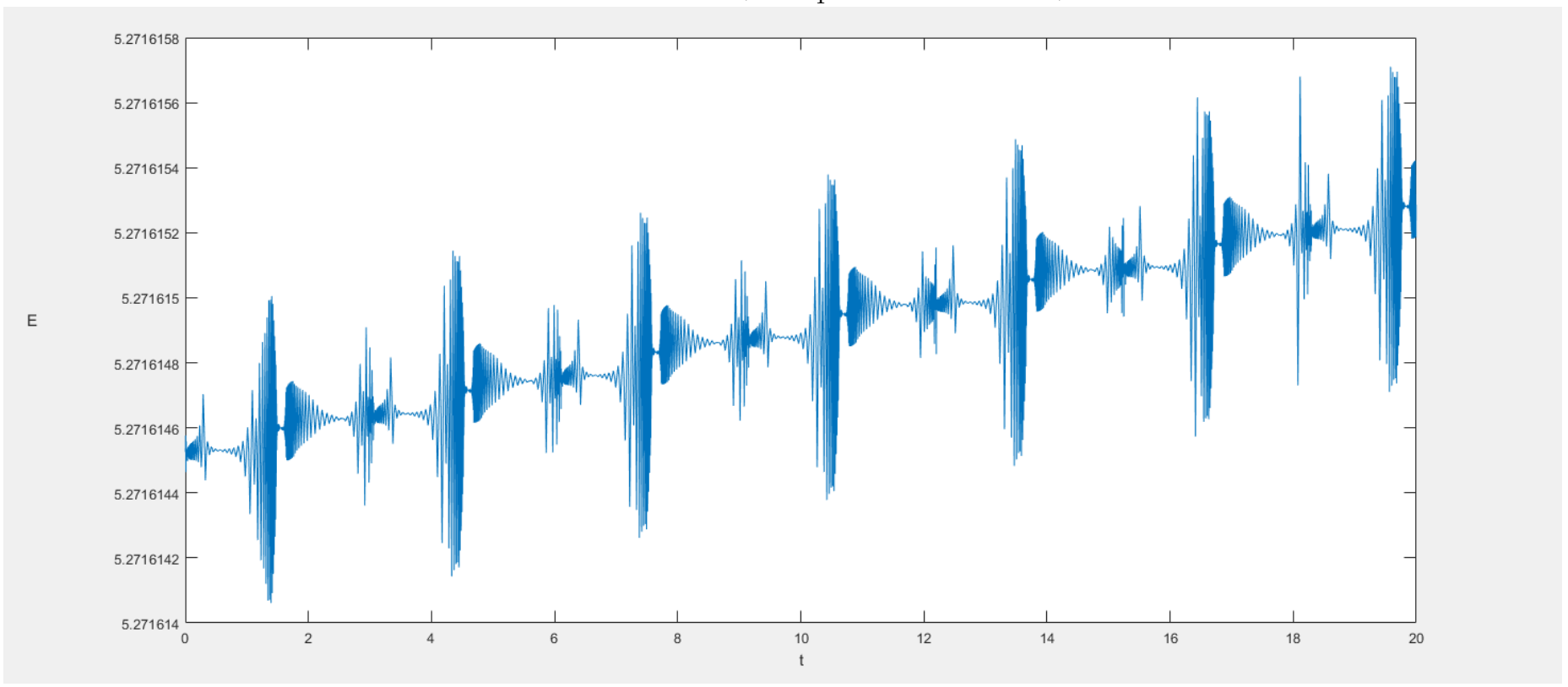

Figure 4.1.18 

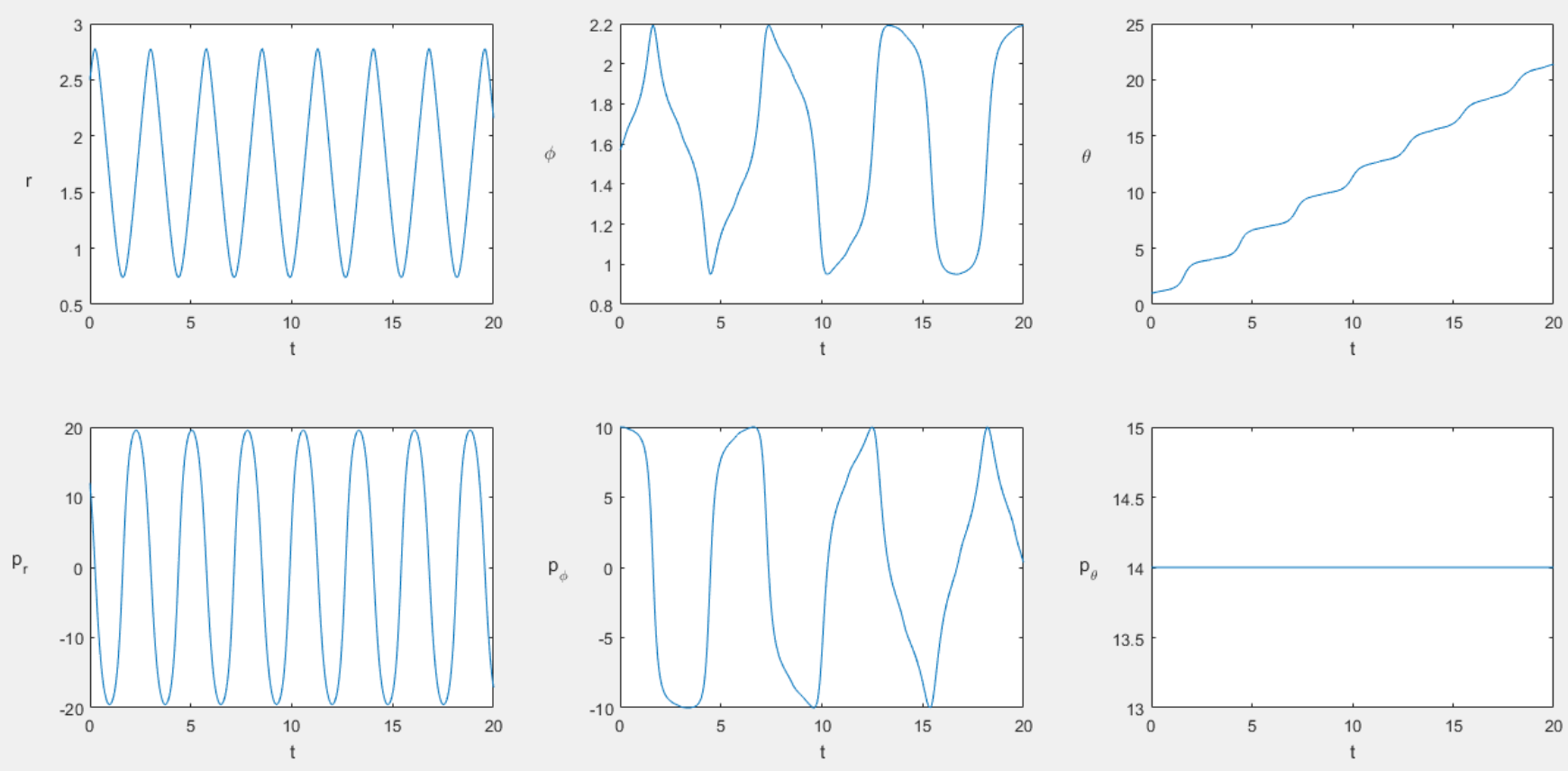

Figure 4.1.19: $\alpha=1.75, \quad \mathcal{H}_{3}\left(2.5, \frac{\pi}{2}, 1,12,10,14\right)=40.87383$

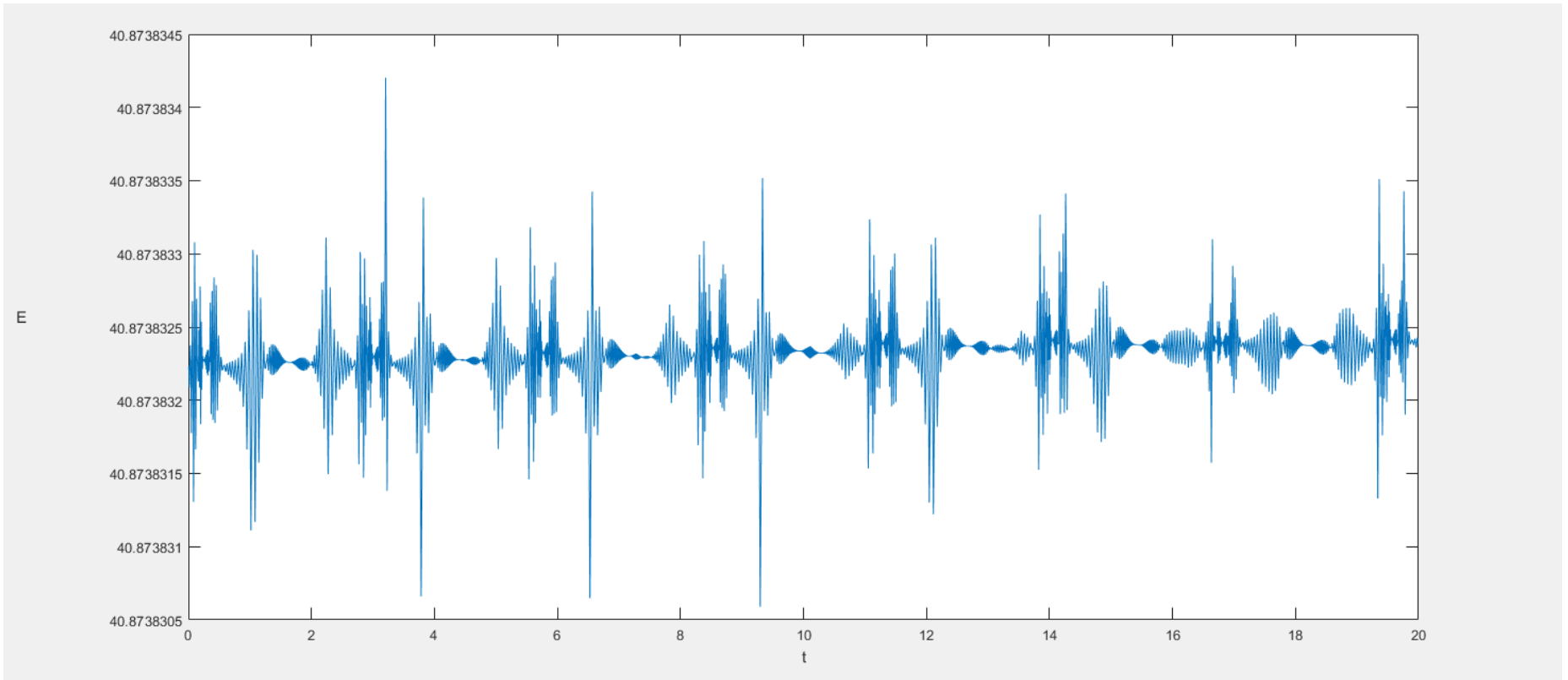

Figure 4.1.20 

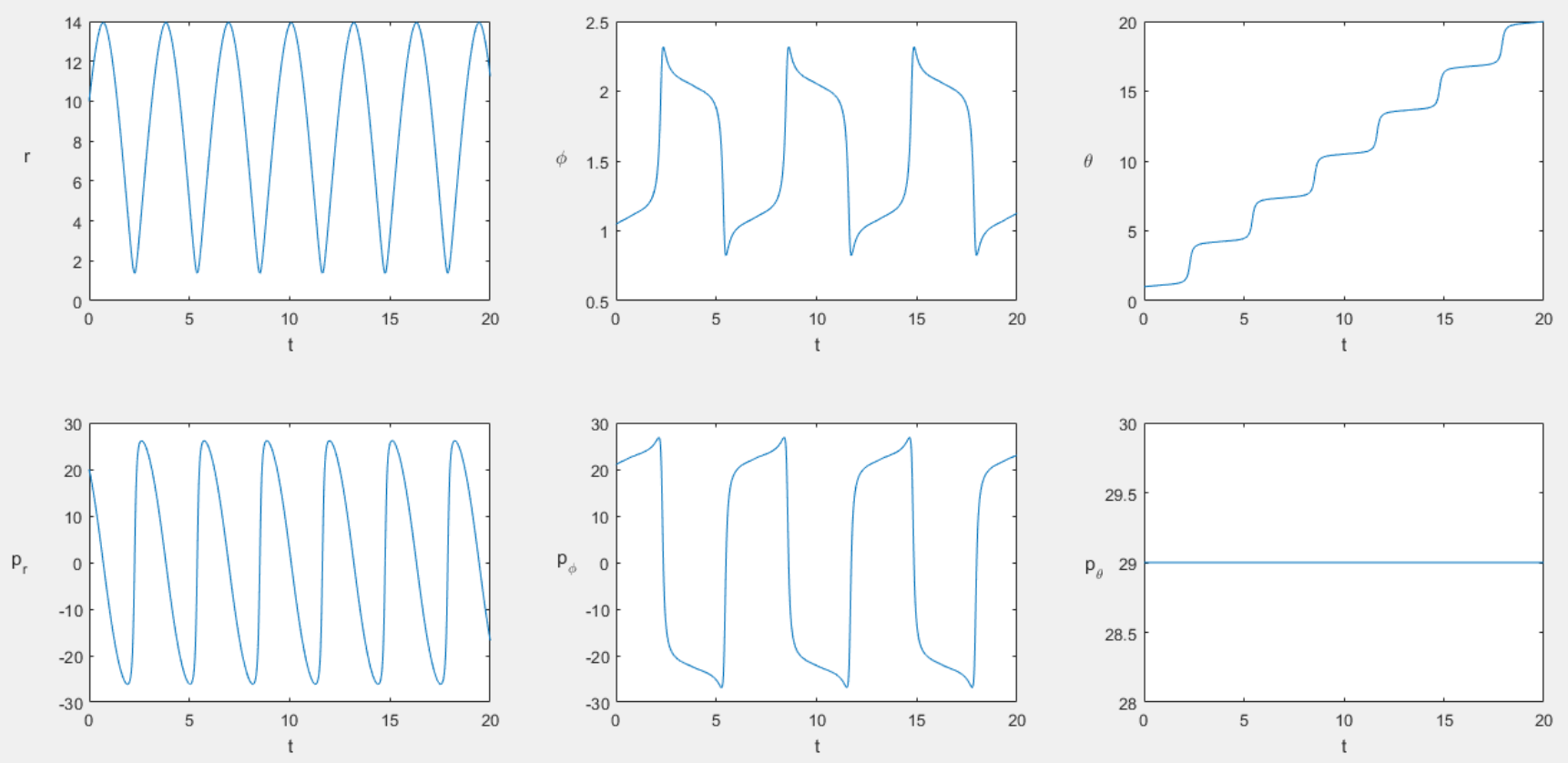

Figure 4.1.21: $\alpha=1.5, \quad \mathcal{H}_{3}\left(10, \frac{\pi}{3}, 1,20,21,29\right)=204.102$

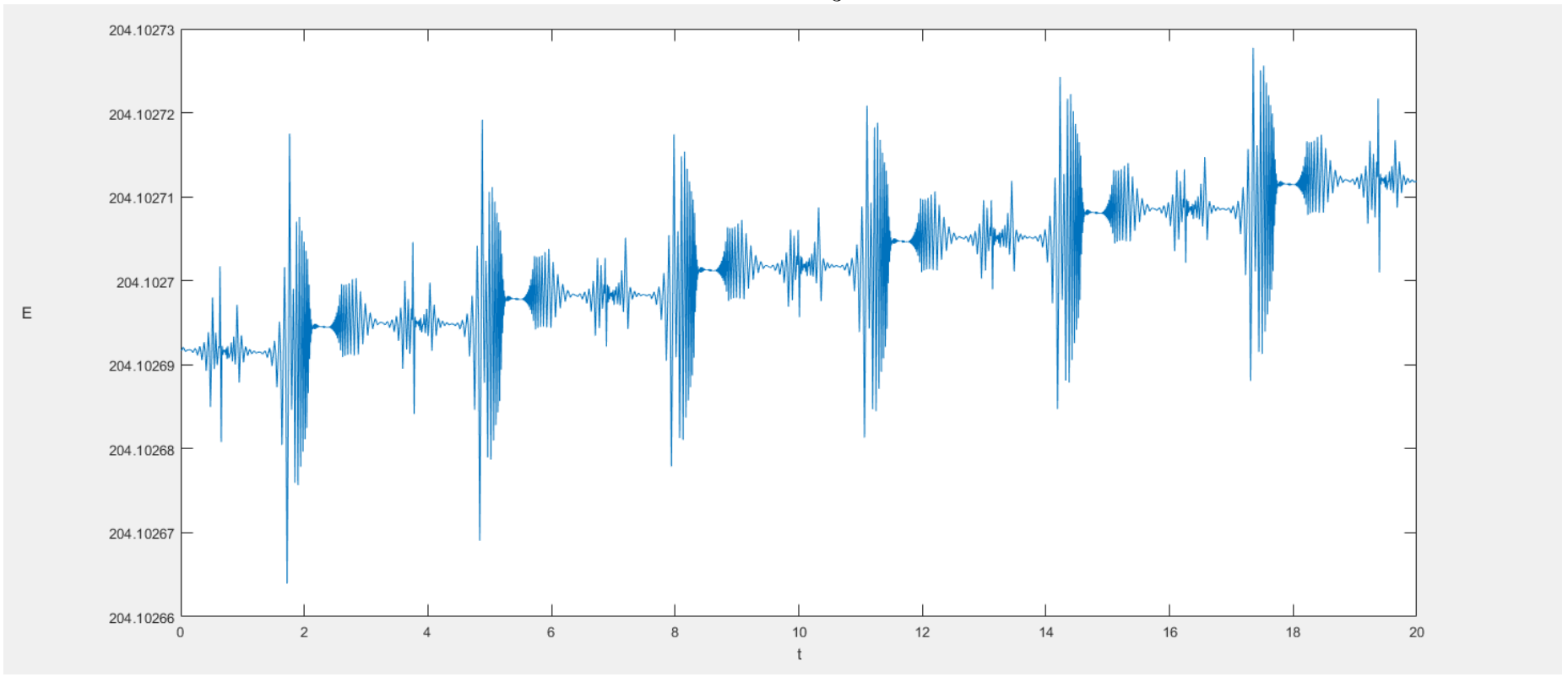

Figure 4.1.22 
Similar to the two-dimensional example, this example demonstrates clear periodic behaviour in the motion. To fully justify this we do as before and investigate the behaviour of the individual coordinates. One may notice that in regard to $r, \theta, p_{r}$ and $p_{\theta}$, the behaviour is largely the same as $r, \vartheta, p_{r}$ and $p_{\vartheta}$ in the two-dimensional case. The same can be said about the corresponding plots for $E$ also. Hence we refer back to Example 4.1.2 for their analysis given that the same conclusions and reasoning can be made.

A new coordinate to discuss however is $\phi$. We notice that it too demonstrates periodic behaviour just like $r$ and $p_{r}$, but with a larger period. The corresponding coordinate $p_{\phi}$ plays a similatr role that $p_{r}$ did with $r$. It shows large change whenever $\phi$ seems to make a large change in direction. The range of $\phi$ seems to be larger with the lower and higher values of $E$. This is also reflected with $p_{\phi}$ showing how with more movement, physically more acceleration can occur. We also recall from the two-dimensional analogue the motion with $r$ and $\vartheta$ being more extreme with larger values and $E$ and so it is natural that in this extended form that $\phi$ follows suit.

Overall the path in the three-dimensional space illustrated by the coordinates seems to be an extension of sorts of what was described in the two-dimensional example. Projecting to the plane mapping $r$ against $\theta$, we obtain very much what we had before in the previous two-dimensional example. However now $\phi$ provides an extra oscillation in the orthogonal direction. Hence we describe the path as the same flower with petals as before but now with vertical orthogonal oscillations.

\subsection{The Kepler Problem}

We begin with a short summary of the Kepler problem mainly following [26, Chapter 3] and [27, Chapter 7.1]. A well-studied problem in classical mechanics is the Kepler problem, or more generally the central force problem. Here one investigates the motion of a particle under the influence of a central force field, i.e. the motion of particles due to forces

$$
\underline{F}(r)=F(r) \underline{e}_{r} .
$$

The above is described using polar coordinates $r$ and $\vartheta$ where $\underline{e}_{r}$ denotes the radial direction. It is defined with respect to an origin known as the center of force so the forces only act along the radius connecting the system to the origin.

A characteristic property about (4.2.1) is that it is dependent only on $r$. Hence the problem is said to be spherically symmetric. This gives the motion to be constrained within a plane. Therefore any three-dimensional problem involving a particle moving due to (4.2.1) can be reduced to an equivalent two-dimensional problem. A further consequence is that the angular momentum, i.e. the conjugate momentum $p_{\vartheta}$ to $\vartheta$, is a conserved quantity. 
Using conservative central fields, i.e.

$$
f(r)=-V^{\prime}(r),
$$

$V$ the potential, one can then use the equations of motion to learn more about the motion of the system and even determine the nature of the orbit.

Comparing back to the three-dimensional example for the Harmonic Oscillator, namely Example 4.1.3, notice we had a potential $V$ dependent only on $r$, i.e. we can find a function $f$ such that $V$ in this example may be determined by an equation of the form (4.2.2). This means that Example 4.1.3 is a central force problem. In fact from equation (4.1.41) we have already seen how this three-dimensional problem may be reduced to an equivalent two-dimensional problem, a characteristic property of the central force problem.

The Kepler problem represents a particular case of the central force problem where the potential is defined as follows:

$$
V(r):=-\frac{k}{r} \quad(\text { three dimensions }), \quad k>0 .
$$

Therefore

$$
f(r)=-\frac{k}{r^{2}}
$$

where $k$ is some positive constant. The minus sign confirms the direction of the force in this case to be towards the center of the system. Due to its structure the force (4.2.4) is also referred to as the attractive inverse square law of force.

As we did for Hamiltonians with the Harmonic Oscillator we would like to form an analogue for the Kepler problem. Here, we choose the Hamilton function to be

$$
\mathcal{H}(q, p)=\psi(p)-F^{-1}\left(\frac{1}{\psi(.)}\right)(q)
$$

where $\psi \in C N\left(\mathbb{R}^{n}\right) \cap C^{1}\left(\mathbb{R}^{n}\right)$ is convex and coercive. The potental term is given by the minus of the inverse Fourier transform of $\frac{1}{\psi(.)}$. For this term to be welldefined the choice of space for which $\frac{1}{\psi(.)}$ is an element of will be important. Further $F^{-1}\left(\frac{1}{\psi(.)}\right)(q)$ is not necessarily continuously differentiable which may be a problem when forming the equations of motion.

We recall our previous choice for the kinetic term to be

$$
\psi(p):=\frac{1}{\alpha}\|p\|^{\alpha}, \quad 1<\alpha \leq 2 .
$$

So that the potential term is well-defined, we read $\frac{1}{\psi(.)}=\alpha\|\cdot\|^{-\alpha}$ as an element in the space of tempered distributions $\mathcal{S}^{\prime}\left(\mathbb{R}^{n}\right)$. Through this we have

$$
F^{-1}\left(\frac{1}{\psi(.)}\right)(q)=\alpha c_{n, \alpha}\|q\|^{\alpha-n}
$$


where $c_{n, \alpha}$ is the constant

$$
c_{n, \alpha}:=\frac{\Gamma\left(\frac{n-\alpha}{2}\right)}{2^{\alpha-\frac{n}{2}} \Gamma\left(\frac{\alpha}{2}\right)} .
$$

The above calculation of the inverse Fourier transform can be found at [24]. In the above $\Gamma$ denotes the Gamma function. For convenience we define $\gamma_{n, \alpha}:=\alpha c_{n, \alpha}$ so that with $\psi$ as at (4.2.6), the Hamilton function appears as

$$
\mathcal{H}(q, p)=\frac{1}{\alpha}\|p\|^{\alpha}-\gamma_{n, \alpha}\|q\|^{\alpha-n} .
$$

For convenience later on it will be useful to define $\tilde{\gamma}_{n, \alpha}:=\alpha \gamma_{n, \alpha}$.

As hinted at before, differentiability with respect to $q$ in (4.2.9) is a problem. It is possible for $\alpha-n$ to be negative and so care must be taken in regard to possible singularities. In examples to come we will make clear any singularities we need to omit from domains of functions so that we may have the required differentiability to study the dynamics.

It holds that $\gamma_{n, \alpha}$ is a positive constant for both $n=2$ and $n=3$. Hence due to the structure of (4.2.9), the conserved value $E$ of the Hamilton function is not always non-negative unlike the situation of Section 4.1 with the Harmonic Oscillator.

Taking $n=3$ and $\alpha=2$ in (4.2.9) one would obtain the Kepler problem in classical mechanics with potential (4.2.3). Transforming to sphercial polar coordinates one would find that the problem can be reduced to an equivalent two-dimensional problem, as in classical mechanics. We will see that this is similarly the case in our dynamics.

However taking $n=1$ at (4.2.9) we would obtain the one-dimensional case which poses a few problems. Firstly, when comparing to mechanics having a onedimensional Kepler problem does not make much sense. The problem is always considered in three-dimensions, with the associated two-dimensional perspective playing a role as previously mentioned. Hence in our dynamics one could argue that this is an original problem without an analogue in classical mechanics.

Secondly there is a problem with the constant $\gamma_{1, \alpha}$. It is negative making the potential of (4.2.9) to be less similar to (4.2.3). Usually, potentials in the Kepler problem decay as $r$ increases. The potential (4.2.3) for the inverse square law is an example. However with the $\gamma_{1, \alpha}$, the potential of (4.2.9) would be growing as $r$ increases. This is more similar to the behaviour of potentials related to the Harmonic Oscillator. Therefore the one-dimensional case might behave differently when compared to its higher dimensional analogues.

Example 4.2.1. We want to study the dynamics associated with $\mathcal{H}_{1, K} \in C^{1}(\mathbb{R} \backslash$ $\{0\} \times \mathbb{R})$ such that

$$
\mathcal{H}_{1, K}(q, p)=\frac{1}{\alpha}|p|^{\alpha}-\gamma_{1, \alpha}|q|^{\alpha-1}, \quad \gamma_{1, \alpha}<0 .
$$


Hamilton's equations follow as

$$
\left\{\begin{array}{l}
\dot{q}=p|p|^{\alpha-2}, \\
\dot{p}=(\alpha-1) \gamma_{1, \alpha} q|q|^{\alpha-3}
\end{array}\right.
$$

A quick remark in regard to possible singularities in the above: looking to the Hamilton function (4.2.10) it is clear that this term is well-defined and continuous on $\mathbb{R}^{2}$ for $1<\alpha \leq 2$. Hence $\mathcal{H}_{1, K} \in C\left(\mathbb{R}^{2}\right)$. However looking to Hamilton's equations we see that the equation for $\dot{p}$ is not well-defined for $q=0$. So because of this we must insist on $\mathcal{H}_{1, K} \in C^{1}(\mathbb{R} \backslash\{0\} \times \mathbb{R})$.

The Hamilton function is conserved determined by the initial conditions i.e. $\mathcal{H}(q(t), p(t))=\mathcal{H}\left(q_{0}, p_{0}\right)=E$ for $q(0)=q_{0}$ and $p(0)=p_{0}$. Through this one can find $\dot{q}$ and $\dot{p}$ to satisfy:

$$
\left\{\begin{array}{l}
\dot{q}= \pm\left(\alpha E+\tilde{\gamma}_{1, \alpha}|q|^{\alpha-1}\right)^{\frac{1}{\alpha^{*}}} \\
\dot{p}= \pm(\alpha-1) \alpha^{\frac{2-\alpha}{\alpha-1}}\left(-\gamma_{1, \alpha}\right)^{\frac{1}{\alpha-1}}\left(\alpha E-|p|^{\alpha}\right)^{\frac{\alpha-2}{\alpha-1}}
\end{array}\right.
$$

As with Example 4.1.1, the sign coefficients in the above solutions are determined by the individual cases $q>0$ and $p \geq 0, q>0$ and $p<0, q<0$ and $p \geq 0, q<0$ and $p<0$. As before this avoids the problem of having to deal with the absolute values at (4.2.11). For the full details we refer to Example B.2 at Appendix B. Through this $q(t)$ and $p(t)$ are solved to be

$$
q(t)=g_{1}^{-1}\left( \pm \alpha^{\frac{3 \alpha-1}{\alpha}} E^{\frac{2 \alpha-1}{\alpha}} t+g_{1}\left(q_{0}\right)\right)
$$

and

$$
p(t)=g_{2}^{-1}\left( \pm\left(-\gamma_{1, \alpha}\right)^{\frac{1}{\alpha-1}}(\alpha-1) E^{\frac{\alpha-2}{\alpha-1}} t+g_{2}\left(p_{0}\right)\right) .
$$

The function $g_{1}: U_{1} \longrightarrow U_{2}, U_{1}, U_{2}$ open in $\mathbb{R}$, is defined as

$$
\begin{aligned}
& g_{1}(q):=\alpha^{2} E|q|{ }_{2} F_{1}\left(\frac{1}{\alpha-1}, \frac{-1}{\alpha} ; \frac{\alpha}{\alpha-1} ; \frac{-\tilde{\gamma}_{1, \alpha}|q|^{\alpha-1}}{\alpha E}\right) \\
& -\tilde{\gamma}_{1, \alpha}|q|^{\alpha}{ }_{2} F_{1}\left(\frac{\alpha-1}{\alpha}, \frac{\alpha}{\alpha-1} ; \frac{2 \alpha-1}{\alpha-1} ; \frac{-\tilde{\gamma}_{1, \alpha}|q|^{\alpha-1}}{\alpha E}\right)
\end{aligned}
$$

and $g_{2}: V_{1} \longrightarrow V_{2}, V_{1}, V_{2}$ also open in $\mathbb{R}$ as

$$
g_{2}(p):=p_{2} F_{1}\left(\frac{\alpha-2}{\alpha-1}, \frac{1}{\alpha} ; \frac{\alpha+1}{\alpha} ; \frac{|p|^{\alpha}}{\alpha E}\right) .
$$

The above solutions are well-defined whenever $|q|<\left(\frac{E}{-\gamma_{1, \alpha}}\right)^{\frac{1}{\alpha-1}}$ and $|p|<(\alpha E)^{\frac{1}{\alpha}}$. 
The corresponding Lagrange function for the Hamilton function (4.2.10) follows as

$$
\mathcal{L}_{1, K}(q, \eta)=\frac{1}{\alpha^{*}}|\eta|^{\alpha^{*}}+\gamma_{1, \alpha}|q|^{\alpha-1}
$$

where for $q$ and $\eta$ fixed, $\mathcal{L}_{1, K}(., \eta) \in C^{1}(\mathbb{R} \backslash\{0\})$ and $\mathcal{L}_{1, K}(q,.) \in C^{2}(\mathbb{R})$. Lagrange's equation follows as

$$
\ddot{q}|\dot{q}|^{\alpha^{*}-2}=\gamma_{1, \alpha}(\alpha-1) q|q|^{\alpha-3} .
$$

Recollecting from Theorem 3.3.2 that $E$ is given by

$$
\frac{\partial \mathcal{L}_{1, K}}{\partial \eta} \eta-\mathcal{L}_{1, K}=E
$$

we can solve $\dot{q}$ to be

$$
\dot{q}= \pm\left(\alpha E+\tilde{\gamma}_{1, \alpha}|q|^{\alpha-1}\right)^{\frac{1}{\alpha^{*}}}
$$

which coincides with the first equation of (4.2.12). Hence, the solution for $q$ would follow as it did when solving Hamilton's equations. The sign coefficient at (4.2.18) is determined as it was in Example 4.1.1, being dependent on the individual cases of sign for $q$ and $\dot{q}$, i.e. $q>0$ and $\dot{q} \geq 0, q>0$ and $\dot{q}<0, q<0$ and $\dot{q} \geq 0, q<0$ and $\dot{q}<0$. We refer to Example B.2 once again for the full details.

We can use the solution for $q$ with (4.2.18) to solve $\dot{q}$. Notice that the condition $|q|<\left(\frac{E}{-\gamma_{\alpha, 1}}\right)^{\frac{1}{\alpha-1}}$ confirms (4.2.18) to be well-defined.

Below are some examples of the phase diagrams for (4.2.11) for selected values of $\alpha$, constructed using Mathematica. The horizontal axis represents the coordinate $q$ where the vertical axis corresponds to $p$. 


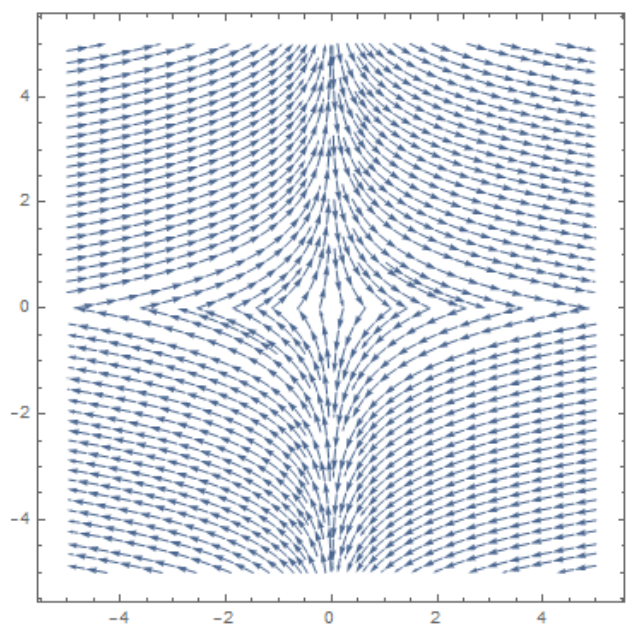

Figure 4.2.1: $\alpha=1.01$

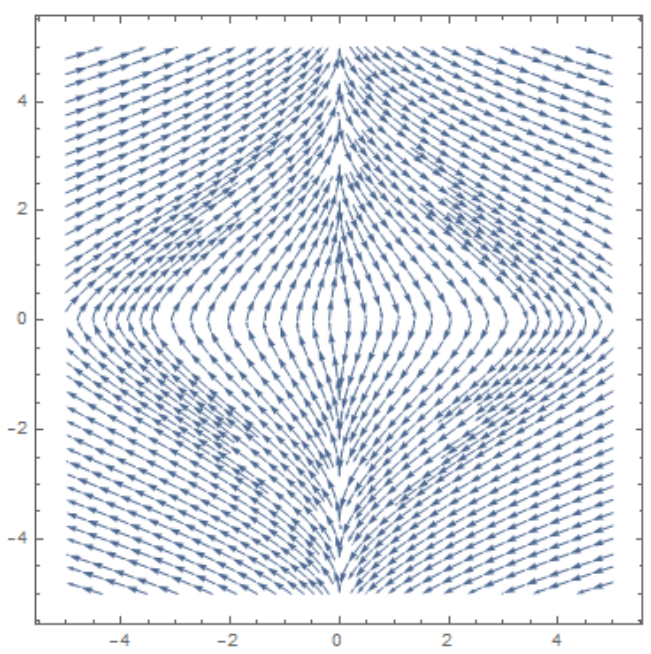

Figure 4.2.3: $\alpha=1.5$

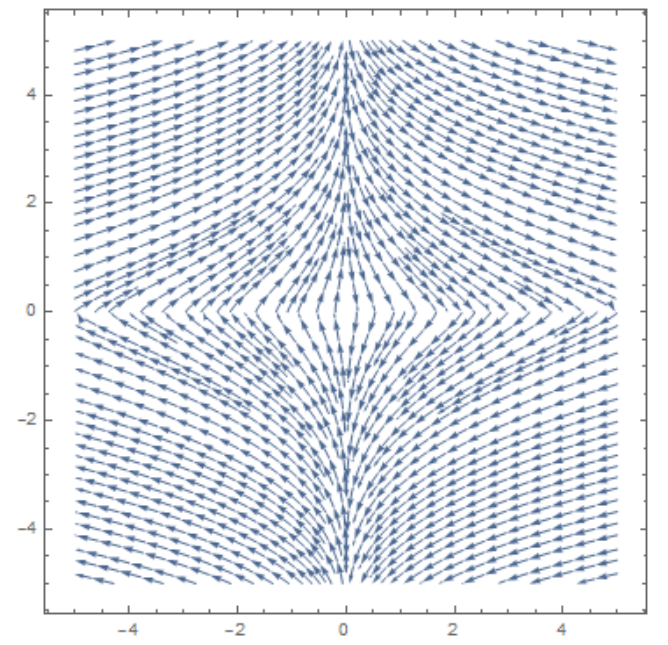

Figure 4.2.2: $\alpha=1.25$

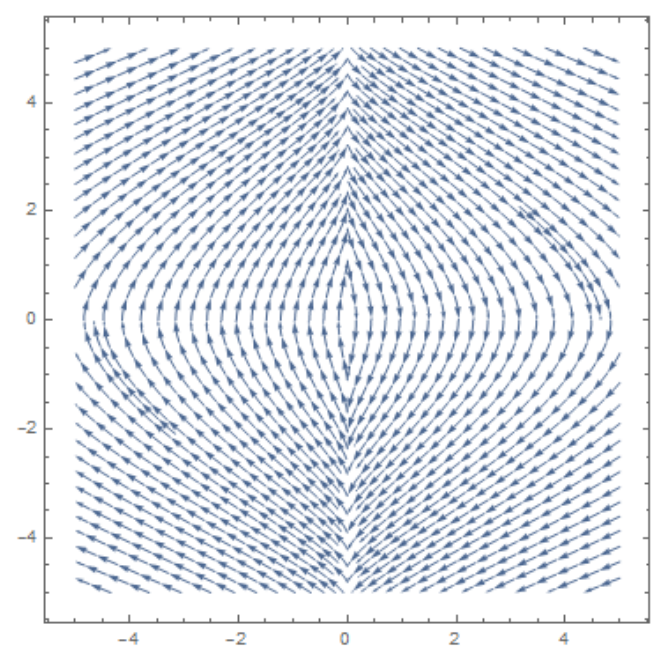

Figure 4.2.4: $\alpha=1.75$

The above figures were constructed as four quartels strung together. To be exact they represent the phase diagrams of the system defined for $q$ and $p$ defined on $[-5,-0.0000001) \cup(0.0000001,5]$.

Observing the above figures we can see that the solutions have a symmetric property, similar to those in Example 4.1.1. Hence similar conclusions can be made here to what was done in that example before so we do not repeat them here.

Example 4.2.2. We want to study the dynamics associated with $\mathcal{H} \in C^{1}\left(\mathbb{R}^{2} \backslash\{0\} \times\right.$ 
$\left.\mathbb{R}^{2}\right)$ such that

$$
\mathcal{H}_{2, K}(q, p)=\frac{1}{\alpha}\|p\|^{\alpha}-\gamma_{2, \alpha}\|q\|^{\alpha-2} .
$$

The associated Hamilton's equations follow as

$$
\left\{\begin{array}{l}
\dot{q}_{j}=p_{j}\|p\|^{\alpha-2}, \\
\dot{p}_{j}=\gamma_{2, \alpha}(\alpha-2) q_{j}\|q\|^{\alpha-4}, \quad j=1,2 .
\end{array}\right.
$$

As with Example 4.1.2, only the main steps are shown. We refer to Example B.3 from Appendix $B$ for the full details.

As before exploiting conserved quantities is of most help. Transforming to polar coordinates the function (4.2.19) is equivalent to $\mathcal{H}_{2, K} \in C^{1}((0, \infty) \times[0,2 \pi) \times \mathbb{R} \backslash$ $\{0\} \times \mathbb{R})$ such that

$$
\mathcal{H}_{2, K}\left(r, \vartheta, p_{r}, p_{\vartheta}\right)=\frac{1}{\alpha}\left(p_{r}^{2}+\frac{p_{\vartheta}^{2}}{r^{2}}\right)^{\frac{\alpha}{2}}-\gamma_{2, \alpha} r^{\alpha-2} .
$$

Hamilton's equations now follow as

$$
\begin{gathered}
\dot{r}=p_{r}\left(p_{r}^{2}+\frac{p_{\vartheta}^{2}}{r^{2}}\right)^{\frac{\alpha-2}{2}}, \\
\dot{\vartheta}=\frac{p_{\vartheta}}{r^{2}}\left(p_{r}^{2}+\frac{p_{\vartheta}^{2}}{r^{2}}\right)^{\frac{\alpha-2}{2}}, \\
\dot{p}_{r}=\frac{p_{\vartheta}^{2}}{r^{3}}\left(p_{r}^{2}+\frac{p_{\vartheta}^{2}}{r^{2}}\right)^{\frac{\alpha-2}{2}}+\gamma_{2, \alpha}(\alpha-2) r^{\alpha-3},
\end{gathered}
$$

and

$$
\dot{p}_{\vartheta}=0
$$

implying that $p_{\vartheta}(t)=\kappa$ for all $t, \kappa$ being some constant. Therefore the corresponding coordinate $p_{\vartheta}$ to the cyclic coordinate $\vartheta$ is conserved as expected from Theorem 3.3.5.

The conserved value of the Hamilton function is determined by the initial conditions i.e. $\mathcal{H}_{2, K}\left(r(t), \vartheta(t), p_{r}(t), p_{\vartheta}(t)\right)=\mathcal{H}_{2, K}\left(r^{0}, \vartheta^{0}, p_{r}^{0}, p_{\vartheta}^{0}\right)=E$. Through this it can be deduced that $p_{r}$ satisfies

$$
p_{r}= \pm \sqrt{\left(\alpha E+\tilde{\gamma}_{2, \alpha} r^{\alpha-2}\right)^{\frac{2}{\alpha}}-\frac{\kappa^{2}}{r^{2}}}
$$

such that

$$
\dot{r}= \pm \sqrt{\left(\alpha E+\tilde{\gamma}_{2, \alpha} r^{\alpha-2}\right)^{\frac{2}{\alpha}}-\frac{\kappa^{2}}{r^{2}}}\left(\alpha E+\tilde{\gamma}_{2, \alpha} r^{\alpha-2}\right)^{\frac{\alpha-2}{\alpha}}
$$


and

$$
\dot{\vartheta}=\frac{\kappa}{r^{2}}\left(\alpha E+\tilde{\gamma}_{2, \alpha} r^{\alpha-2}\right)^{\frac{\alpha-2}{\alpha}} .
$$

Then $r$ is found to satisfy

$$
\gamma+t= \pm \int \frac{r\left(\alpha E+\tilde{\gamma}_{2, \alpha} r^{\alpha-2}\right)^{\frac{2-\alpha}{\alpha}}}{\sqrt{r^{2}\left(\alpha E+\tilde{\gamma}_{2, \alpha} r^{\alpha-2}\right)^{\frac{2}{\alpha}}-\kappa^{2}}} \mathrm{~d} r
$$

where $\gamma$ is a constant obtained through integration. Assuming the integration to be possible $r$ may be determined in terms of $t$ only. The constant of integration may be eliminated as before through the introduction of initial conditions.

We may then use this solution for $r$ with (4.2.27) to obtain a first-order differential equation of only $\vartheta$. This equation may then be integrated to find $\vartheta$ introducing a further constant of integration. This constant may then be eliminated as before using initial conditions.

The corresponding Lagrange function is

$$
\mathcal{L}(q, \eta)=\frac{1}{\alpha^{*}}\|\eta\|^{\alpha^{*}}+\gamma_{2, \alpha}\|q\|^{\alpha-2},
$$

$\mathcal{L}(., \eta) \in C^{1}\left(\mathbb{R}^{2} \backslash\{0\}\right)$ and $\mathcal{L}(q,.) \in C^{2}\left(\mathbb{R}^{2}\right)$. Lagrange's equations follow as

$$
\ddot{q}_{j}\|\dot{q}\|^{\alpha^{*}-2}+\left(\alpha^{*}-2\right) \dot{q}_{j}\|\dot{q}\|^{\alpha^{*}-4} \sum_{l=1}^{2} \dot{q}_{l} \ddot{q}_{l}=\gamma_{2, \alpha}(\alpha-2) q_{j}\|q\|^{\alpha-4}, \quad j=1, \ldots, n .
$$

Transforming the function at (4.2.29) to polar coordinates gives a new function

$$
\mathcal{L}_{2, K}(r, \vartheta, \dot{r}, \dot{\vartheta})=\frac{1}{\alpha^{*}}\left(\dot{r}^{2}+r^{2} \dot{\vartheta}^{2}\right)^{\frac{\alpha^{*}}{2}}+\gamma_{2, \alpha} r^{\alpha-2}
$$

$\mathcal{L}_{2, K}(., ., \dot{r}, \dot{\vartheta}) \in C^{1}((0, \infty) \times[0,2 \pi))$ and $\mathcal{L}_{2, K}(r, \vartheta, .,.) \in C^{2}\left(\mathbb{R}^{2}\right)$. Lagrange's equations for (4.2.30) follow as

$$
\frac{\mathrm{d}}{\mathrm{d} t}\left(\dot{r}\left(\dot{r}^{2}+r^{2} \dot{\vartheta}^{2}\right)^{\frac{\alpha^{*}-2}{2}}\right)=r \dot{\vartheta}^{2}\left(\dot{r}^{2}+r^{2} \dot{\vartheta}^{2}\right)^{\frac{\alpha^{*}-2}{2}}+\gamma_{2, \alpha}(\alpha-2) r^{\alpha-3}
$$

and

$$
\frac{\mathrm{d}}{\mathrm{d} t}\left(r^{2} \dot{\vartheta}\left(\dot{r}^{2}+r^{2} \dot{\vartheta}^{2}\right)^{\frac{\alpha^{*}-2}{2}}\right)=0
$$

implying that $p_{\vartheta}(t)=\kappa$ for all $t, \kappa$ being some constant. Therefore with $\vartheta$ being a cyclic coordinate of (4.2.30), the claim of Theorem 3.3.6 is satisfied. 
Recollecting from Theorem 3.3.2 that E may be given by

$$
\frac{\partial \mathcal{L}_{2, K}}{\partial \dot{r}} \dot{r}+\frac{\partial \mathcal{L}_{2, K}}{\partial \dot{\vartheta}} \dot{\vartheta}-\mathcal{L}_{2, K}=E
$$

this gives

$$
\dot{r}= \pm \sqrt{\left(\alpha E+\tilde{\gamma}_{2, \alpha} r^{\alpha-2}\right)^{\frac{2}{\alpha}}-\frac{\kappa^{2}}{r^{2}}}\left(\alpha E+\tilde{\gamma}_{2, \alpha} r^{\alpha-2}\right)^{\frac{\alpha-2}{\alpha}}
$$

and

$$
\dot{\vartheta}=\frac{\kappa}{r^{2}}\left(\alpha E+\tilde{\gamma}_{2, \alpha} r^{\alpha-2}\right)^{\frac{\alpha-2}{\alpha}} .
$$

Comparing the above to (4.2.26) and (4.2.27) we see that the solutions can now be defined as they were with the Hamilton function. Having done this, equations (4.2.32) and (4.2.33) can then be used to solve for $\dot{r}$ and $\dot{\vartheta}$ respectively.

As with the examples for the Harmonic Oscillator, there are some conditions we need to impose such that everything is well-defined:

(i) $\alpha E+\tilde{\gamma}_{2, \alpha} r^{\alpha-2}>0$

(ii) $r^{2}\left(\alpha E+\tilde{\gamma}_{2, \alpha} r^{\alpha-2}\right)^{\frac{2}{\alpha}}-\kappa^{2}>0$.

We deal with the above conditions as we did in Example 4.1.2. Through this we see that it is sufficent to have $p_{r} \neq 0$ for the above to hold.

As was discussed in the introduction of this chapter, we have a solution in the form of (4.2.28) does not tell us much about its behaviour. Hence with the help of Matlab below are figures presenting plots approximating the evolution of the coordinates satisfying Hamilton's equations and the value E. In each case it is specified in the captions the specific value of $\alpha$ as well as the corresponding initial values that were taken. 

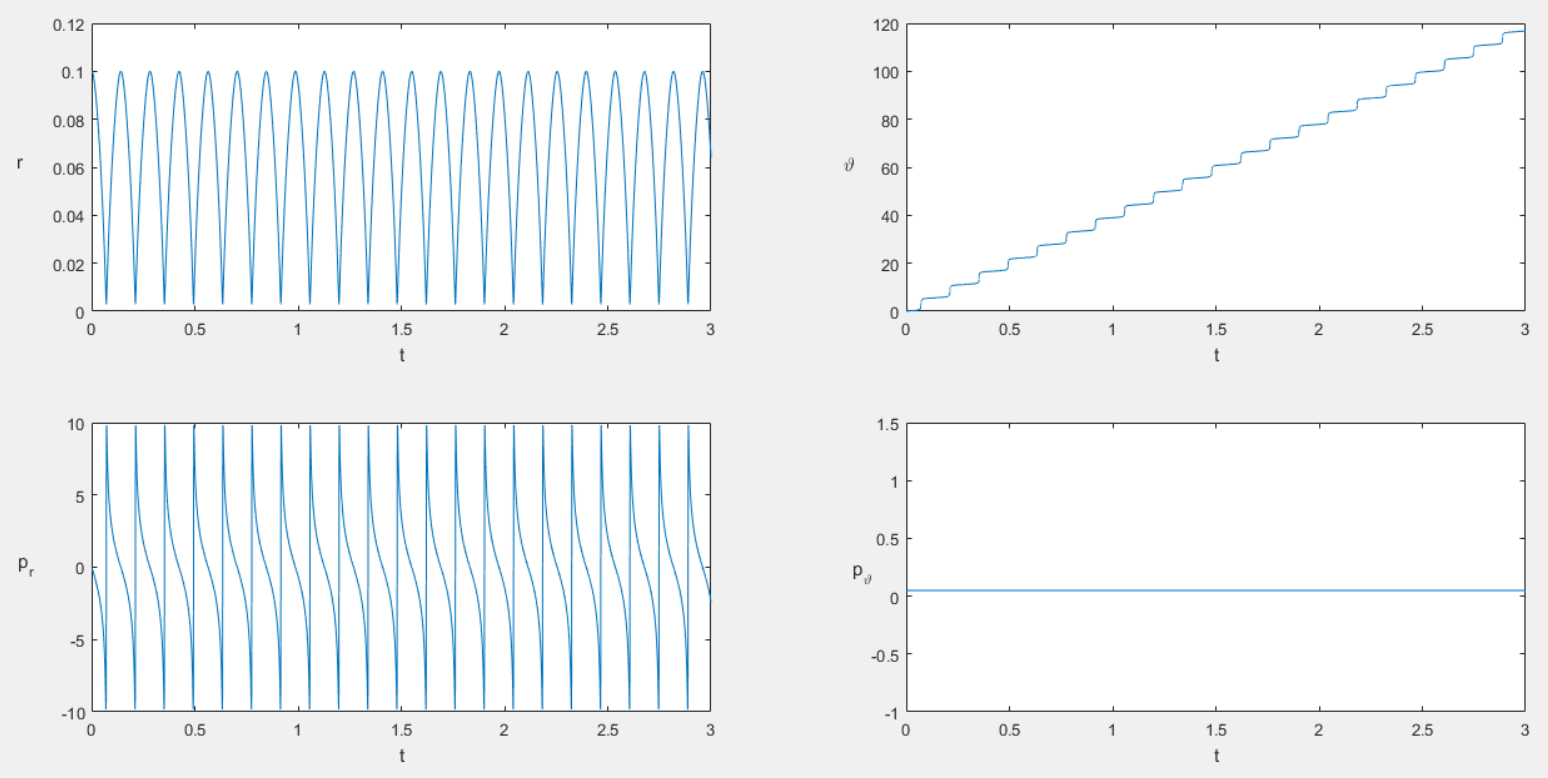

Figure 4.2.5: $\alpha=1.5, \quad \mathcal{H}_{2, K}(0.1,0.07,0.03,0.05)=-9.6873$

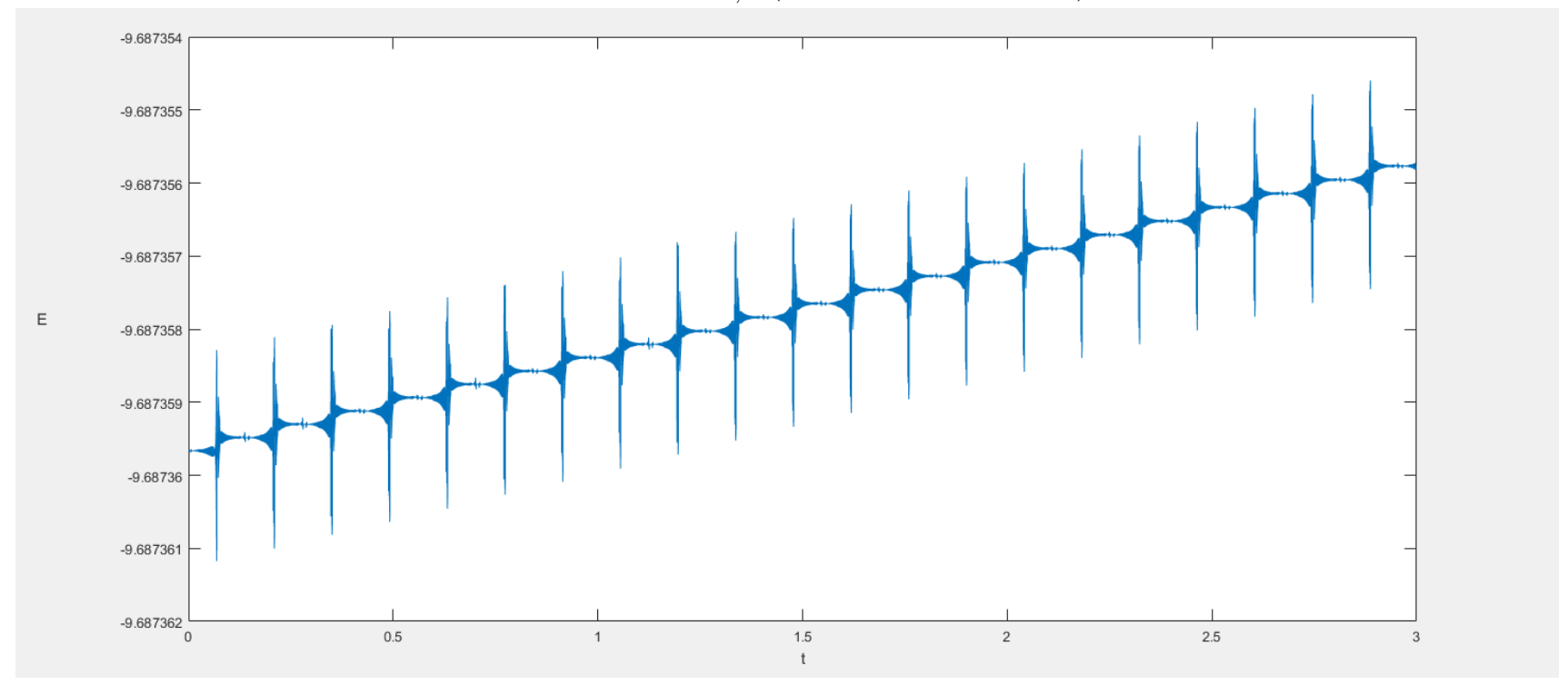

Figure 4.2.6 

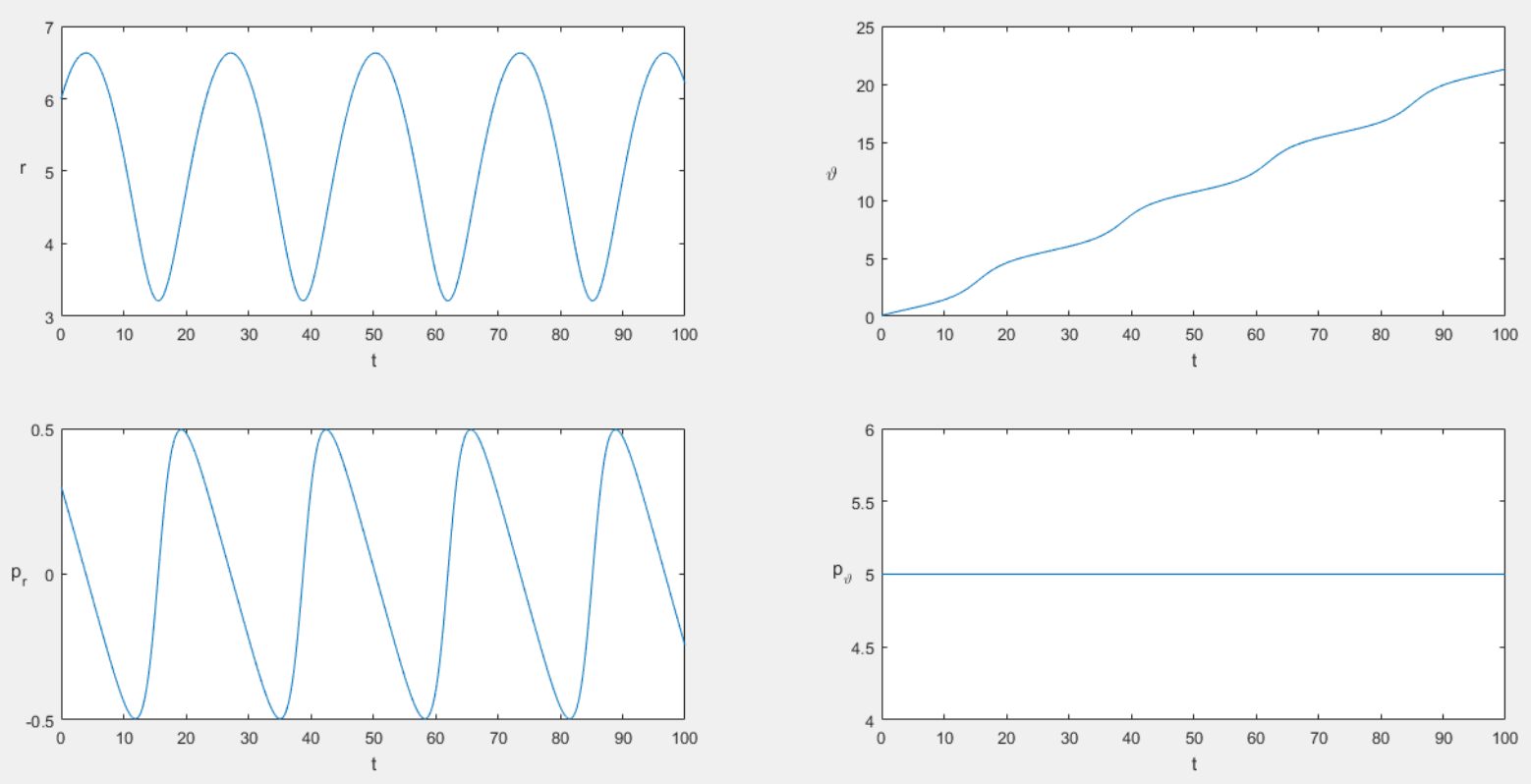

Figure 4.2.7: $\alpha=1.75, \quad \mathcal{H}_{2, K}(6,0.07,0.3,5)=-4.134802$

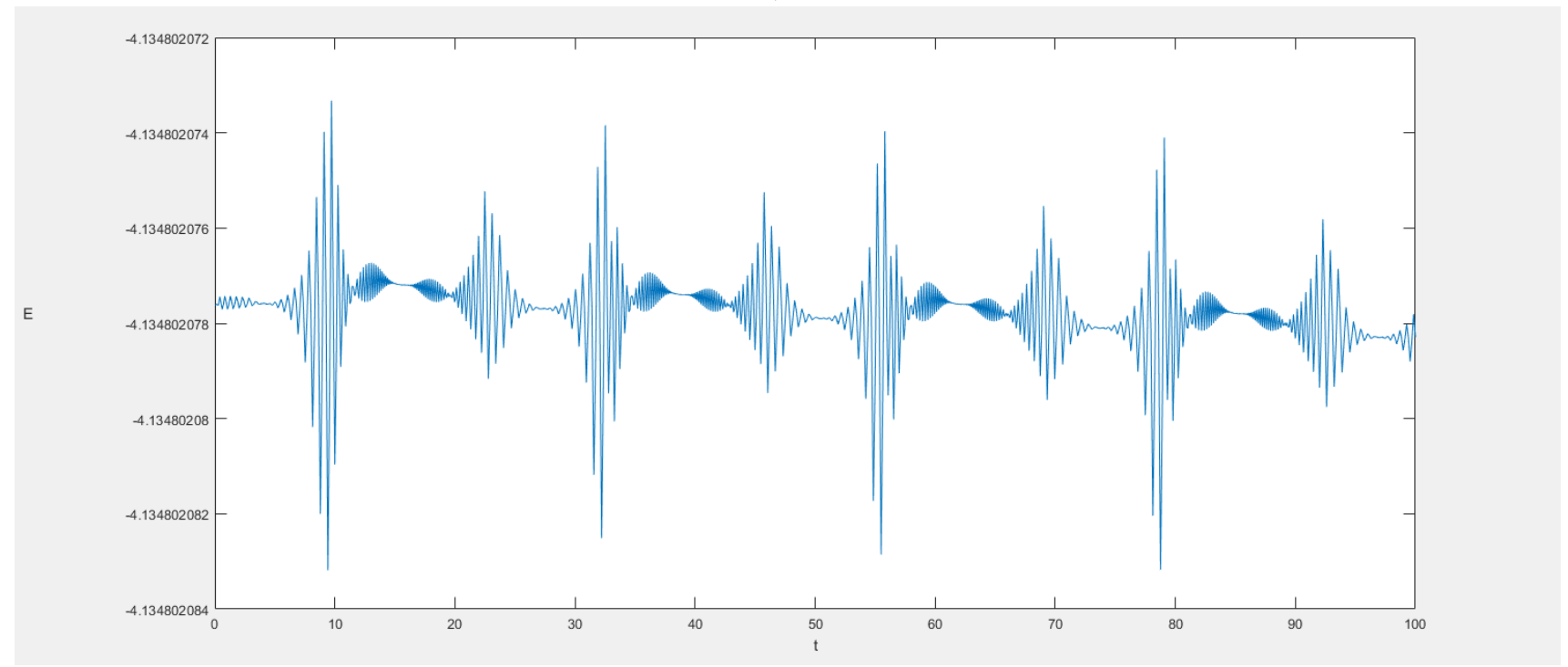

Figure 4.2 .8 

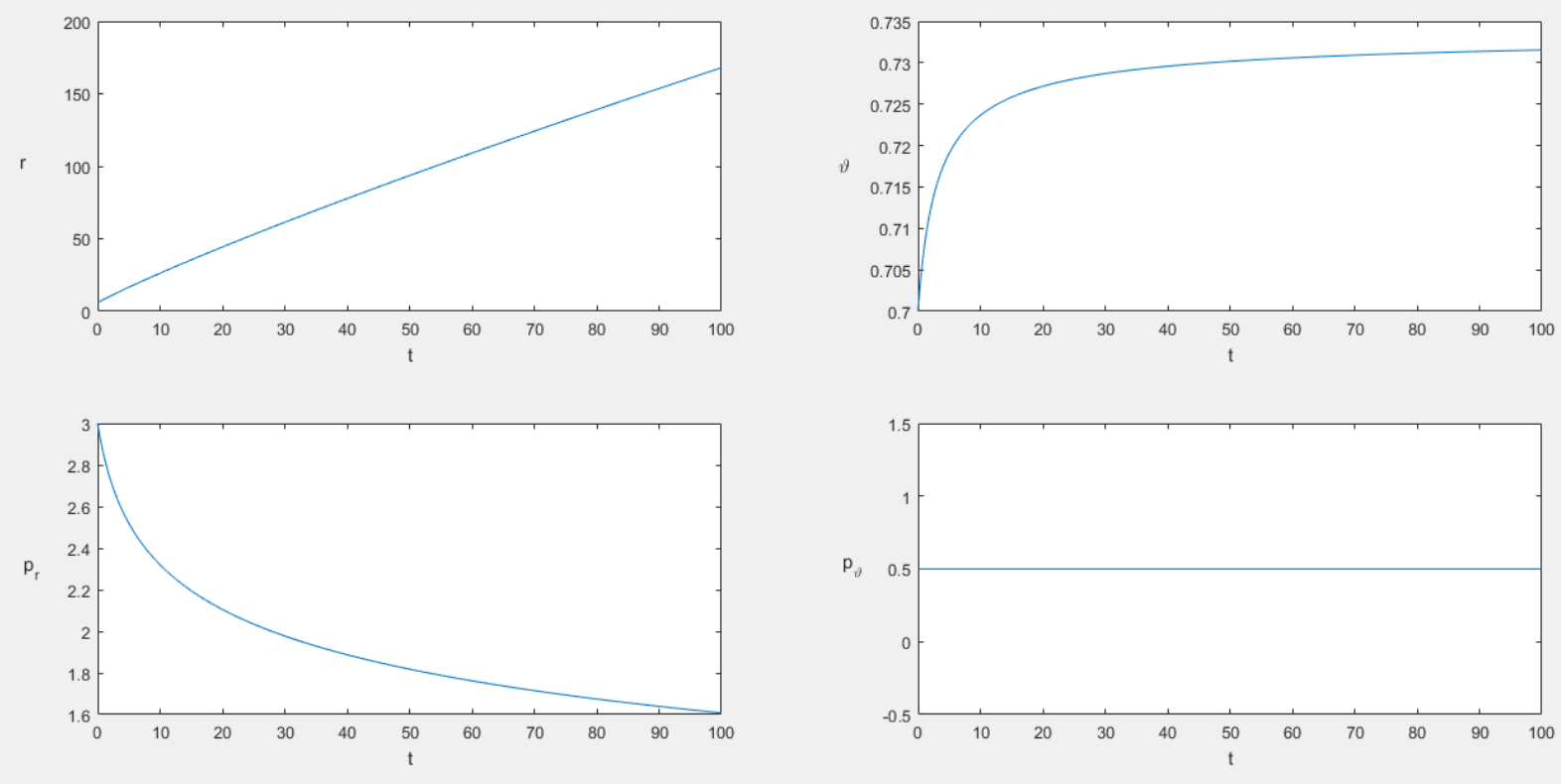

Figure 4.2.9: $\alpha=1.75, \quad \mathcal{H}_{2, K}(6,0.7,3,0.5)=-0.686503$

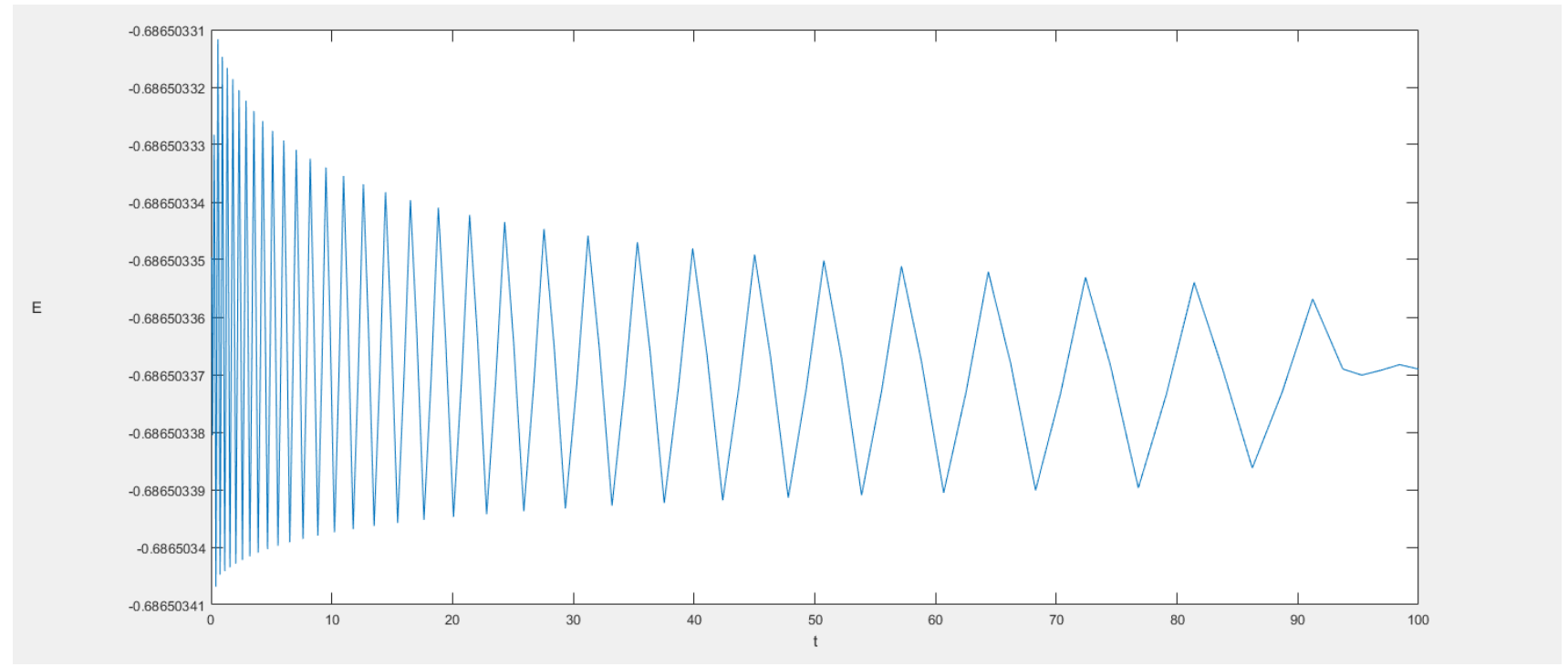

Figure 4.2.10 

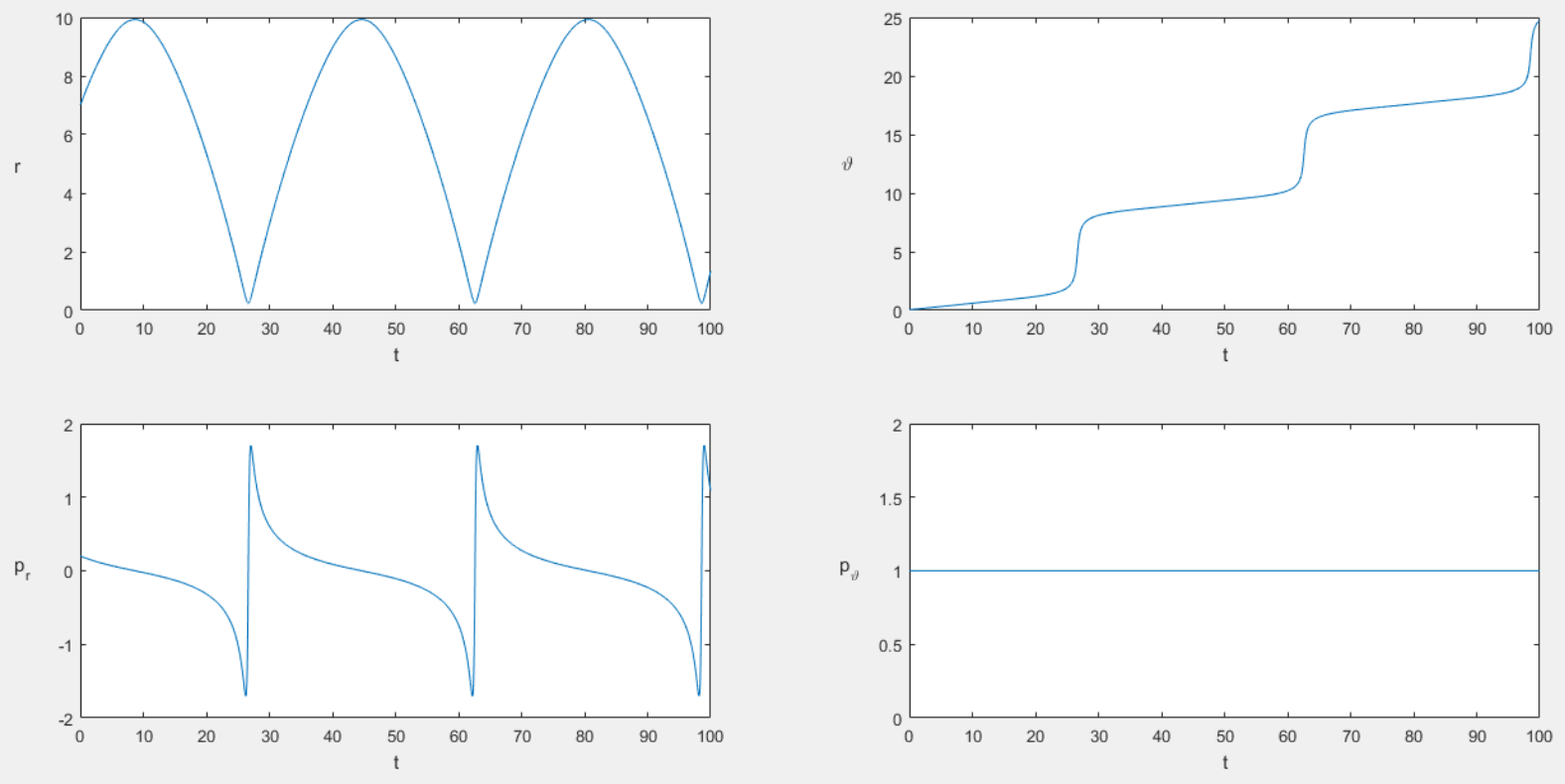

Figure 4.2.11: $\alpha=1.25, \quad \mathcal{H}_{2, K}(7,0.05,0.2,1)=-0.265155$

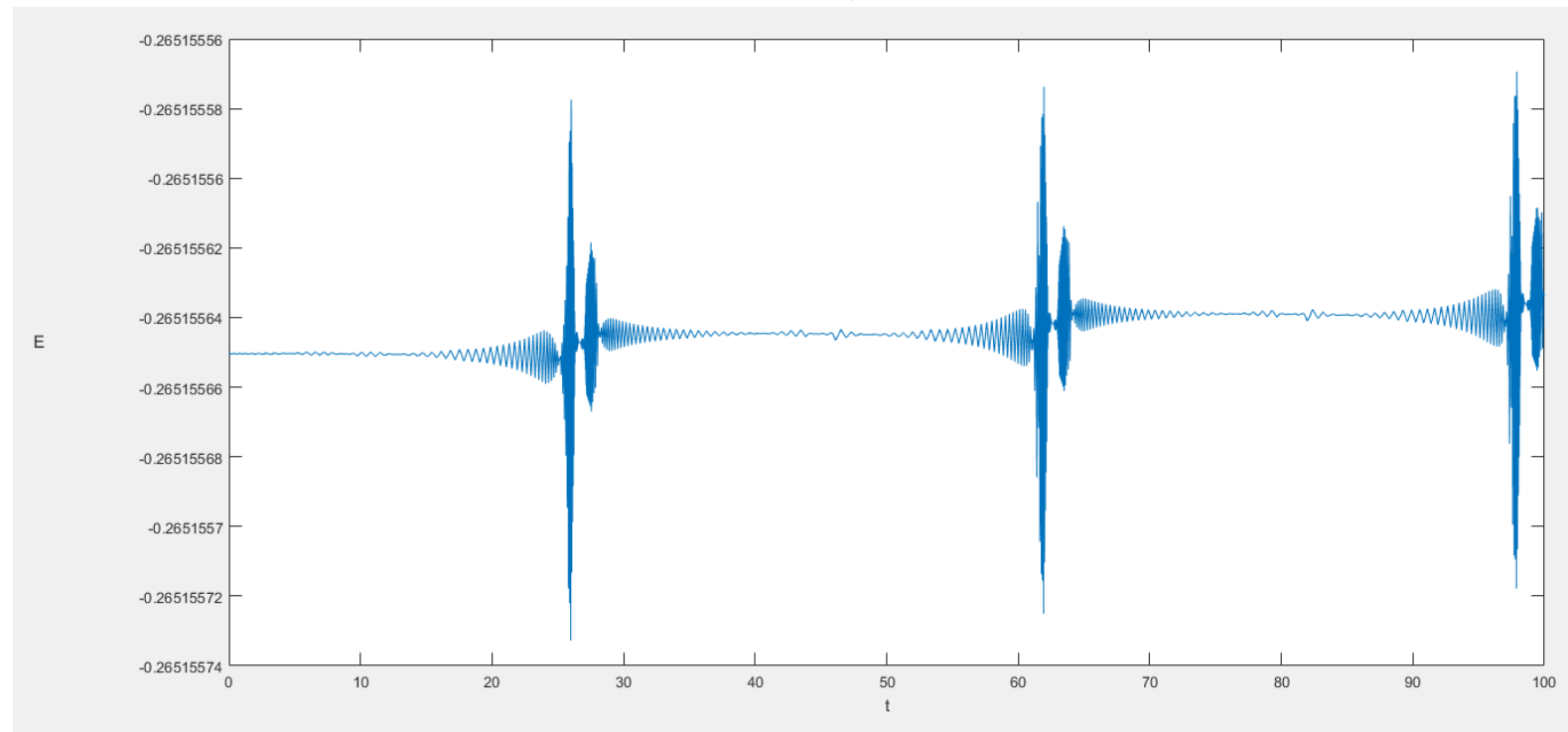

Figure 4.2 .12 

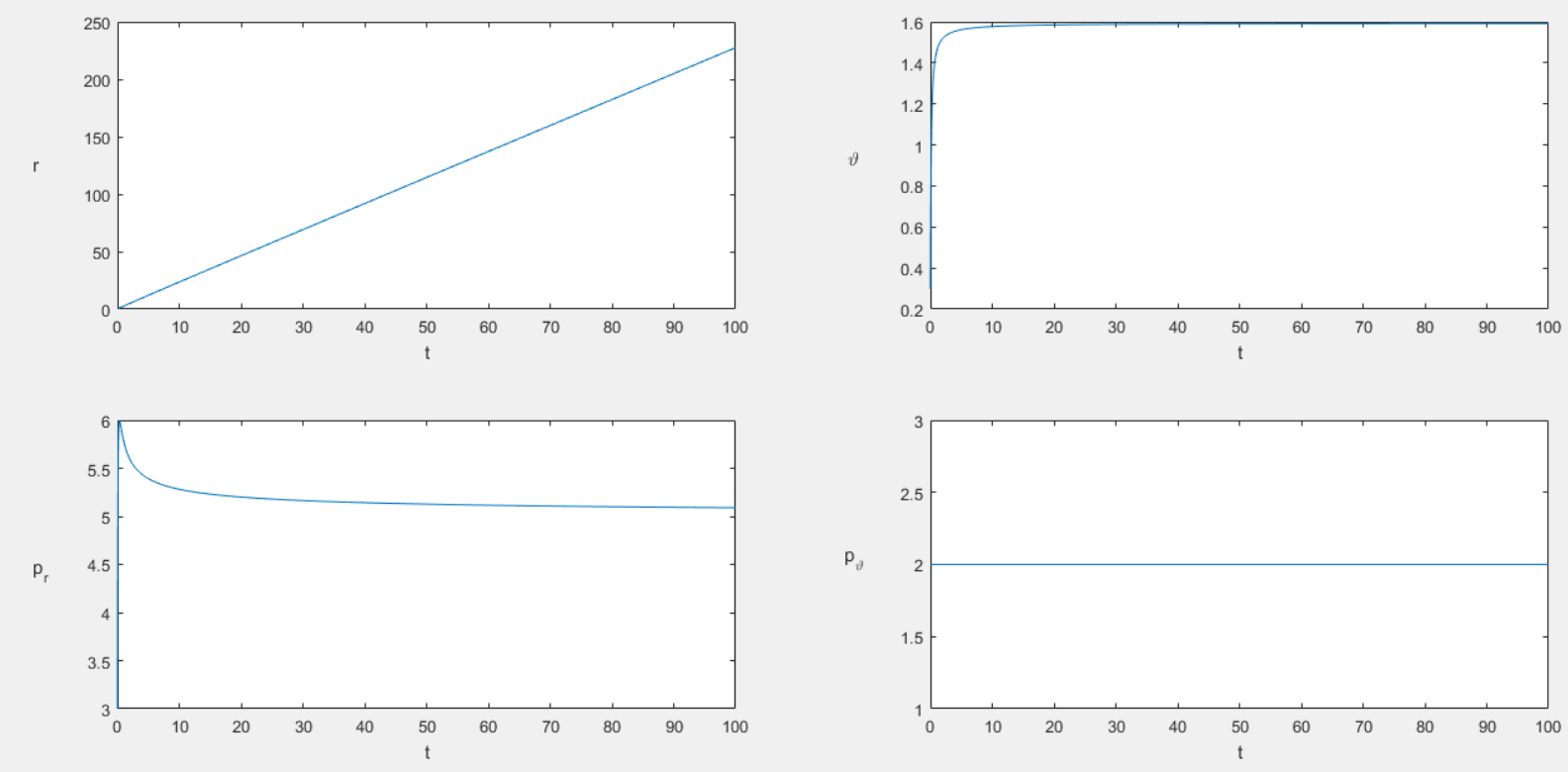

Figure 4.2.13: $\alpha=1.5, \quad \mathcal{H}_{2, K}(0.3,0.3,3,2)=7.44813$

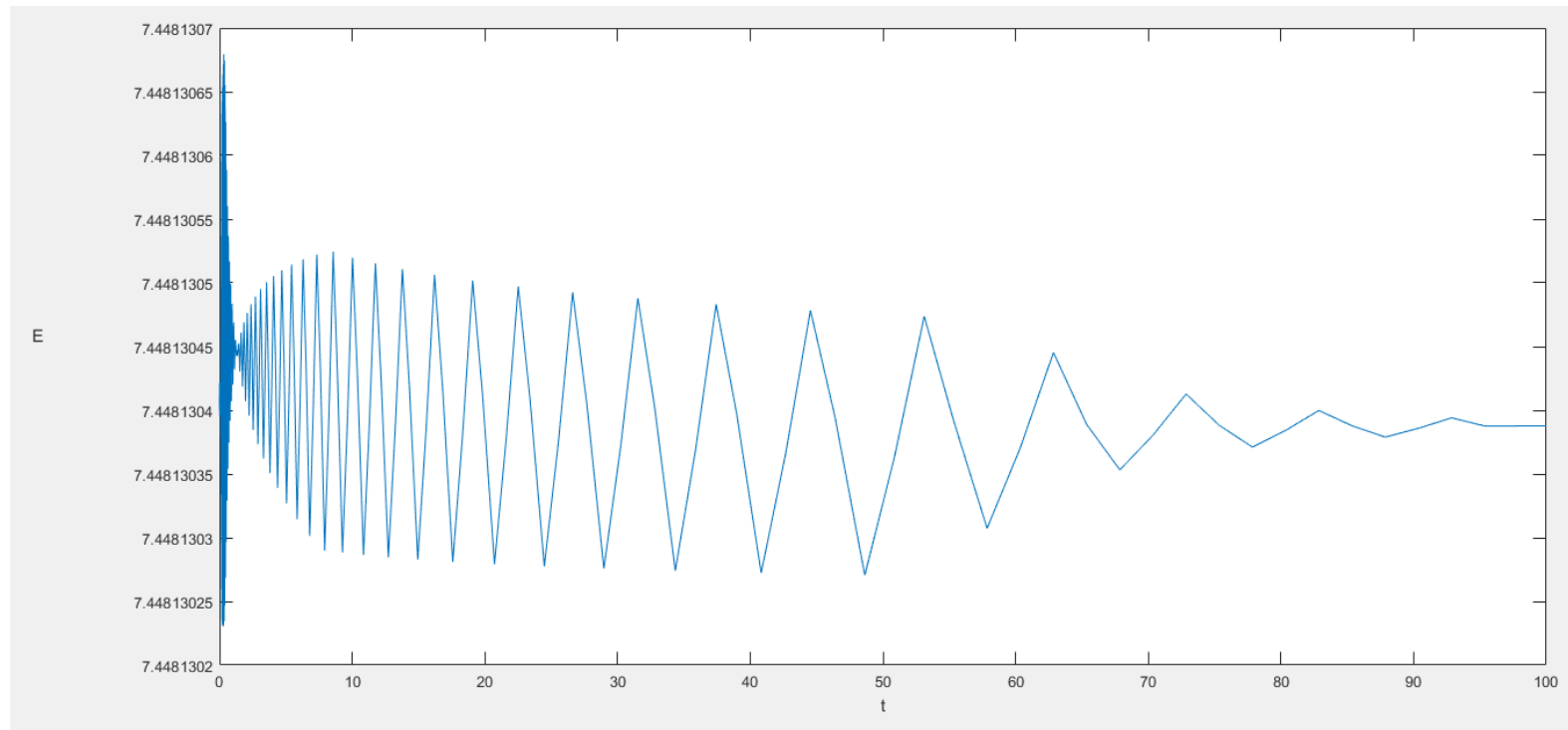

Figure 4.2.14 

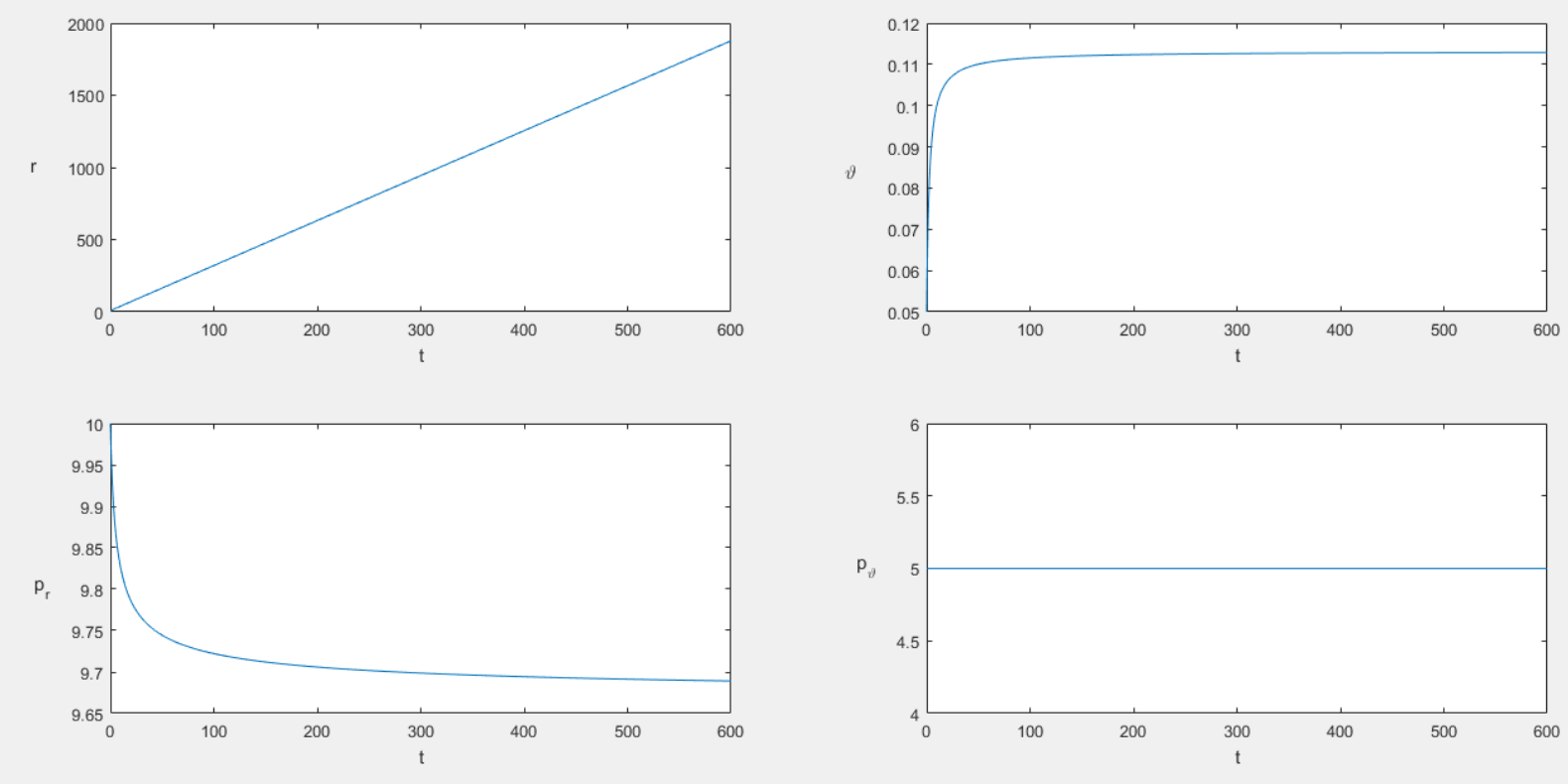

Figure 4.2.15: $\alpha=1.5, \quad \mathcal{H}_{2, K}(8,0.05,10,5)=20.03408$

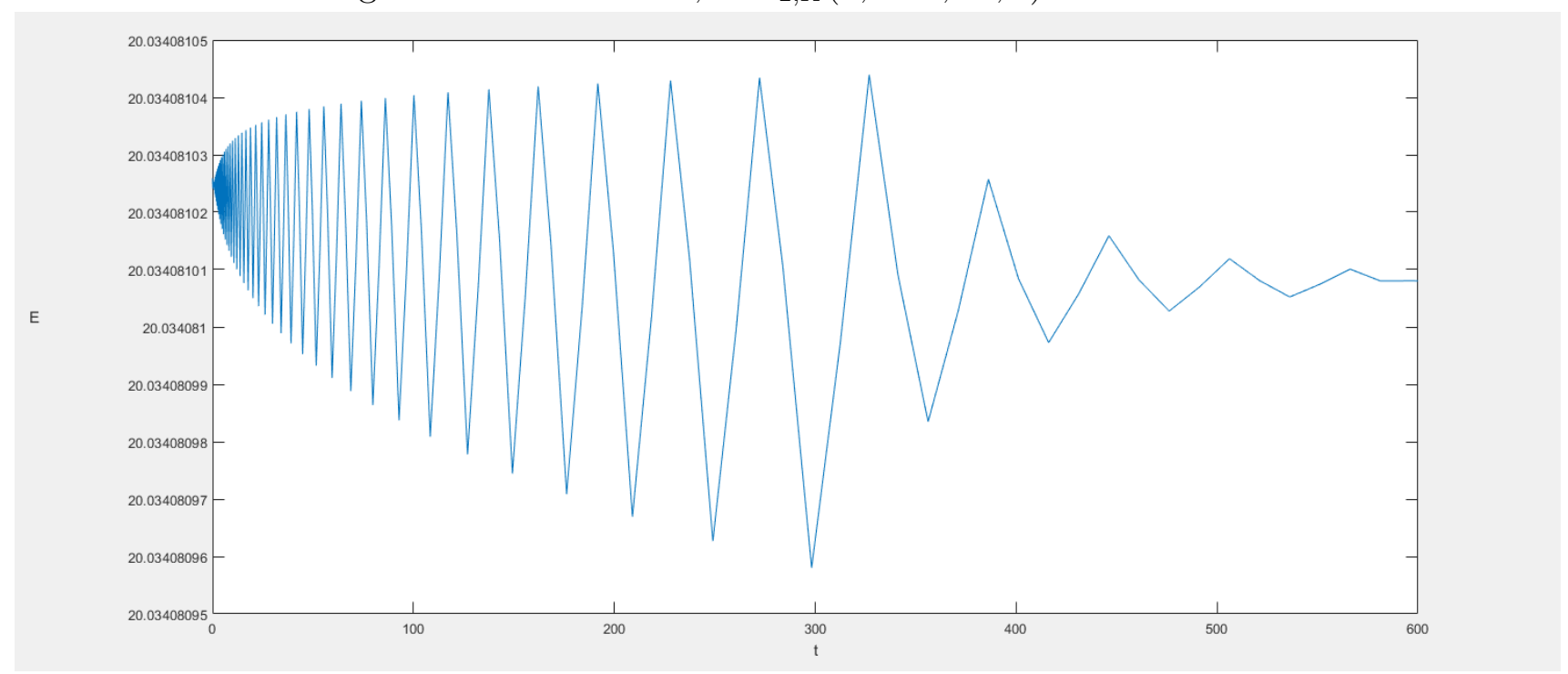

Figure 4.2.16 
Looking to the above figures there are two clear distinct cases. More positive $E$ indicates an unbounded motion, where the more negtaive values of $E$ illustrate oscillatory motion, similar to what we had with the Harmonic Oscillator. This change in behaviour seems to occur somewhere when $E$ is close to zero, as seen with Figures 4.2.9 and 4.2.11. Similar to what was done with the Harmonic Oscillator we shall go through each coordinate in turn explaining why such behaviours are suggested. Additionally in the case of the oscillatory motion we shall refer mainly back to the examples of the Harmonic Oscillator as the analysis that can be made here is largely the same as what has been said before. However any distinctions worth mentioning will be covered. We now begin discussing the case of unbounded motion before moving on afterwards to the oscillatory case.

Let us quickly mention the simplest obeservation first, common to all of the figures. We notice again the coordinate $p_{\vartheta}$ to be constant. This is again unsurprising given the differential equation (4.2.24).

To concentrate on the unbounded case we refer to Figures 4.2.9, 4.2.13 and 4.2.15. Most notable of this is the consistent increasing of $r$. This immediately eliminates any oscillatory properties, suggesting the motion works away from the origin of the configuration. Looking to the plots of $p_{r}$ and $\vartheta$ we can learn more about how exactly this radius increases.

The plot of $\vartheta$ initially increases at a very rapid rate before evening out. This suggests a very swift circular motion around the starting point to start with the radius of the circle always increasing with every rotation. As E increases maximum value of $\vartheta$ seems to increase as we see up to Figure 4.2.13. However we then have a decrease in Figure 4.2.15 where there is higher energy. This seems to further suggest the rapid change in the motion initially before the evening out towards the end.

The plot of $p_{r}$ also supports the behaviour so far suggested. Initially it is very high, demonstrating the large change initially but it decreases with high values of $t$. This suggests physically the motion stabilising and slowing down. There are even some cases where it increases quickly to almost its highest point at the start, see Figure 4.2.13.

Finally the corresponding plots of $E$ in Figures 4.2.10, 4.2.14 and 4.2.16 all share a similar pattern. Each have large oscillations initially demonstrating the quick rotations at the start. The energy then evens out with large time as the motion itself calms with large values of $t$.

Overall the trace of the motion illustrated by the polar coordinates in the twodimenional plane seems to be the shape of a spiral. It starts with radius $r_{0}$ and expands its way outwards continuously before evening out.

Moving on to the oscillatory case we now look to Figures 4.2.5, 4.2.7 and 4.2.11. Overall the motion suggested is mostly the same as what was discussed in the twodimensional example for the Harmonic Oscillator. Here the motion seemed to be 
similar to a flower with petals. This differences however is that $r$ here seems to oscillate more frequently with lesser values as $E$ tends to $-\infty$. Additionally $\vartheta$ seems to fluctuate more wildly with lower energies. This suggests that with more negative energy we obtain more frequent and shorter petals for the flower where as E gets larger the petals become thinner with taller heights.

Example 4.2.3. We want to study the dynamics associated with the function $\mathcal{H} \in$ $C^{1}\left(\mathbb{R}^{3} \backslash\{0\} \times \mathbb{R}^{3}\right)$ such that

$$
\mathcal{H}(q, p)=\frac{1}{\alpha}\|p\|^{\alpha}-\gamma_{3, \alpha}\|q\|^{\alpha-3}
$$

Hamilton's equations follow as

$$
\left\{\begin{array}{l}
\dot{q}_{j}=p_{j}\|p\|^{\alpha-2}, \\
\dot{p}_{j}=\gamma_{3, \alpha}(\alpha-3) q_{j}\|q\|^{\alpha-5}, \quad j=1,2,3 .
\end{array}\right.
$$

As before having more conserved quantities is useful hence we transform to spherical polar coordinates. Through this we obtain a new Hamilton function which we define by $\mathcal{H}_{3, K} \in C^{1}\left((0, \infty) \times[0, \pi] \times[0,2 \pi) \times \mathbb{R} \backslash\{0\} \times \mathbb{R}^{2}\right)$ such that

$$
\mathcal{H}_{3, K}\left(r, \phi, \theta, p_{r}, p_{\phi}, p_{\theta}\right)=\frac{1}{\alpha}\left(p_{r}^{2}+\frac{p_{\phi}^{2}}{r^{2}}+\frac{p_{\theta}^{2}}{r^{2} \sin ^{2} \phi}\right)^{\frac{\alpha}{2}}-\gamma_{3, \alpha} r^{\alpha-3} .
$$

Hamilton's equations are as follows:

$$
\begin{gathered}
\dot{r}=p_{r}\left(p_{r}^{2}+\frac{p_{\phi}^{2}}{r^{2}}+\frac{p_{\theta}^{2}}{r^{2} \sin ^{2} \phi}\right)^{\frac{\alpha-2}{2}} \\
\dot{\phi}=\frac{p_{\phi}}{r^{2}}\left(p_{r}^{2}+\frac{p_{\phi}^{2}}{r^{2}}+\frac{p_{\theta}^{2}}{r^{2} \sin ^{2} \phi}\right)^{\frac{\alpha-2}{2}} \\
\dot{\theta}=\frac{p_{\theta}}{r^{2} \sin ^{2} \phi}\left(p_{r}^{2}+\frac{p_{\phi}^{2}}{r^{2}}+\frac{p_{\theta}^{2}}{r^{2} \sin ^{2} \phi}\right)^{\frac{\alpha-2}{2}} \\
\dot{p}_{r}=\frac{1}{r^{3}}\left(p_{\phi}^{2}+\frac{p_{\theta}^{2}}{\sin ^{2} \phi}\right)\left(p_{r}^{2}+\frac{p_{\phi}^{2}}{r^{2}}+\frac{p_{\theta}^{2}}{r^{2} \sin ^{2} \phi}\right)^{\frac{\alpha-2}{2}}+\gamma_{3, \alpha}(\alpha-3) r^{\alpha-4} \\
\dot{p}_{\phi}=\frac{p_{\theta}^{2} \cos \phi}{r^{2} \sin ^{3} \phi}\left(p_{r}^{2}+\frac{p_{\phi}^{2}}{r^{2}}+\frac{p_{\theta}^{2}}{r^{2} \sin ^{2} \phi}\right)^{\frac{\alpha-2}{2}}
\end{gathered}
$$

and

$$
\dot{p}_{\theta}=0 \text {, }
$$


implying that $p_{\theta}(t)=\beta_{3}$ for all $t, \beta_{3}$ being some constant. With $\theta$ being a cyclic coordinate of (4.2.36), this confirms the claim of Theorem 3.3.5.

The Hamilton function (4.2.36), $\mathcal{H}_{3, K}$ may be considered as an equivalent function of only four variables $\tilde{\mathcal{H}}_{3, K} \in C^{1}((0, \infty) \times[0,2 \pi) \times \mathbb{R} \backslash\{0\} \times \mathbb{R})$ given by

$$
\tilde{\mathcal{H}}_{3, K}\left(r, \vartheta, p_{r}, p_{\vartheta}\right)=\frac{1}{\alpha}\left(p_{r}^{2}+\frac{\beta_{2}^{2}}{r^{2}}\right)^{\frac{\alpha}{2}}-\gamma_{3, \alpha} r^{\alpha-3}
$$

where

$$
\beta_{2}^{2}=p_{\phi}^{2}+\frac{\beta_{3}^{2}}{\sin ^{2} \phi} .
$$

This illustrates the property of the central force problem in classical mechanics where a three-dimensional problem may be reduced to an equivalent two-dimensional problem.

As with Example 4.1.3, to fully obtain the solutions for this problem we need to use the theory of Hamilton-Jacobi. Hence again we simply list here the main results and refer to Exampe 6.1.3 of Chapter 6 for the full details.

From the theory of Hamilton-Jacobi, we find that $\beta_{2}$ is contant such that

$$
r^{2}\left(\left(\alpha E+\tilde{\gamma}_{3, \alpha} r^{\alpha-3}\right)^{\frac{2}{\alpha}}-p_{r}^{2}\right)=p_{\phi}^{2}+\frac{\beta_{3}^{2}}{\sin ^{2} \phi}=\beta_{2}^{2} .
$$

This gives the solutions to be:

$$
\begin{aligned}
& p_{r}= \pm \sqrt{\left(\alpha E+\tilde{\gamma}_{3, \alpha} r^{\alpha-3}\right)^{\frac{2}{\alpha}}-\frac{\beta_{2}^{2}}{r^{2}}} \\
& p_{\phi}= \pm \sqrt{\beta_{2}^{2}-\frac{\beta_{3}^{2}}{\sin ^{2} \phi}} \\
& p_{\theta}=\beta_{3}, \\
& \gamma_{1}+t= \pm \int \frac{r\left(\alpha E+\tilde{\gamma}_{3, \alpha} r^{\alpha-3}\right)^{\frac{2-\alpha}{\alpha}}}{\sqrt{r^{2}\left(\alpha E+\tilde{\gamma}_{3, \alpha} r^{\alpha-3}\right)^{\frac{2}{\alpha}}-\beta_{2}^{2}}} \mathrm{~d} r \\
& \gamma_{2} \pm \int \frac{\beta_{2}}{r \sqrt{r^{2}\left(\alpha E+\tilde{\gamma}_{3, \alpha} r^{\alpha-3}\right)^{\frac{2}{\alpha}}-\beta_{2}^{2}}} \mathrm{~d} r= \pm \int \frac{\beta_{2} \sin \phi}{\sqrt{\beta_{2}^{2} \sin ^{2} \phi-\beta_{3}^{2}}} \mathrm{~d} \phi
\end{aligned}
$$

and

$$
\gamma_{3} \pm \int \frac{\beta_{3}}{\sin \phi \sqrt{\beta_{2}^{2} \sin ^{2} \phi-\beta_{3}^{2}}} \mathrm{~d} \phi=\theta
$$


where $\gamma_{i}, i=1,2,3$, are constants of integration. As with previous examples $r$ may be found explicitly in terms of through (4.2.45) whenever the integration is possible. The constant $\gamma_{1}$ may then be eliminated with initial conditions.

The remaining coordinates $\phi$ and $\theta$ may be found as before by using equations (4.2.46) and (4.2.47). Substituting $r$ and eliminating additional constants as we integrate obtains $\phi$ by (4.2.46). Repeating the process using both $r$ and $\phi$ with (4.2.47) solves $\theta$.

As with the two-dimensional case, there are some conditions we must check so that everything in the example so far is well-defined:

(i) $\alpha E+\tilde{\gamma}_{3, \alpha} r^{\alpha-3}>0$,

(ii) $r^{2}\left(\alpha E+\tilde{\gamma}_{3, \alpha} r^{\alpha-3}\right)^{\frac{2}{\alpha}}-\beta_{2}^{2}>0$,

(iii) $\beta_{2}^{2}-\frac{\beta_{3}^{2}}{\sin ^{2} \phi}>0$.

We can follow a similar method to what was seen Example 4.1.3 to deduce that $p_{r} \neq 0$ is sufficient for all three of the above to hold.

As was discussed in the introduction of this chapter, we have solutions with forms that do not tell us much about their behaviour. Hence with the help of Matlab below are figures presenting plots approximating the evolution of the solutions of Hamilton's equations and the value E. In each case it is specified in the captions the specific value of $\alpha$ as well as the corresponding initial values that were taken. 

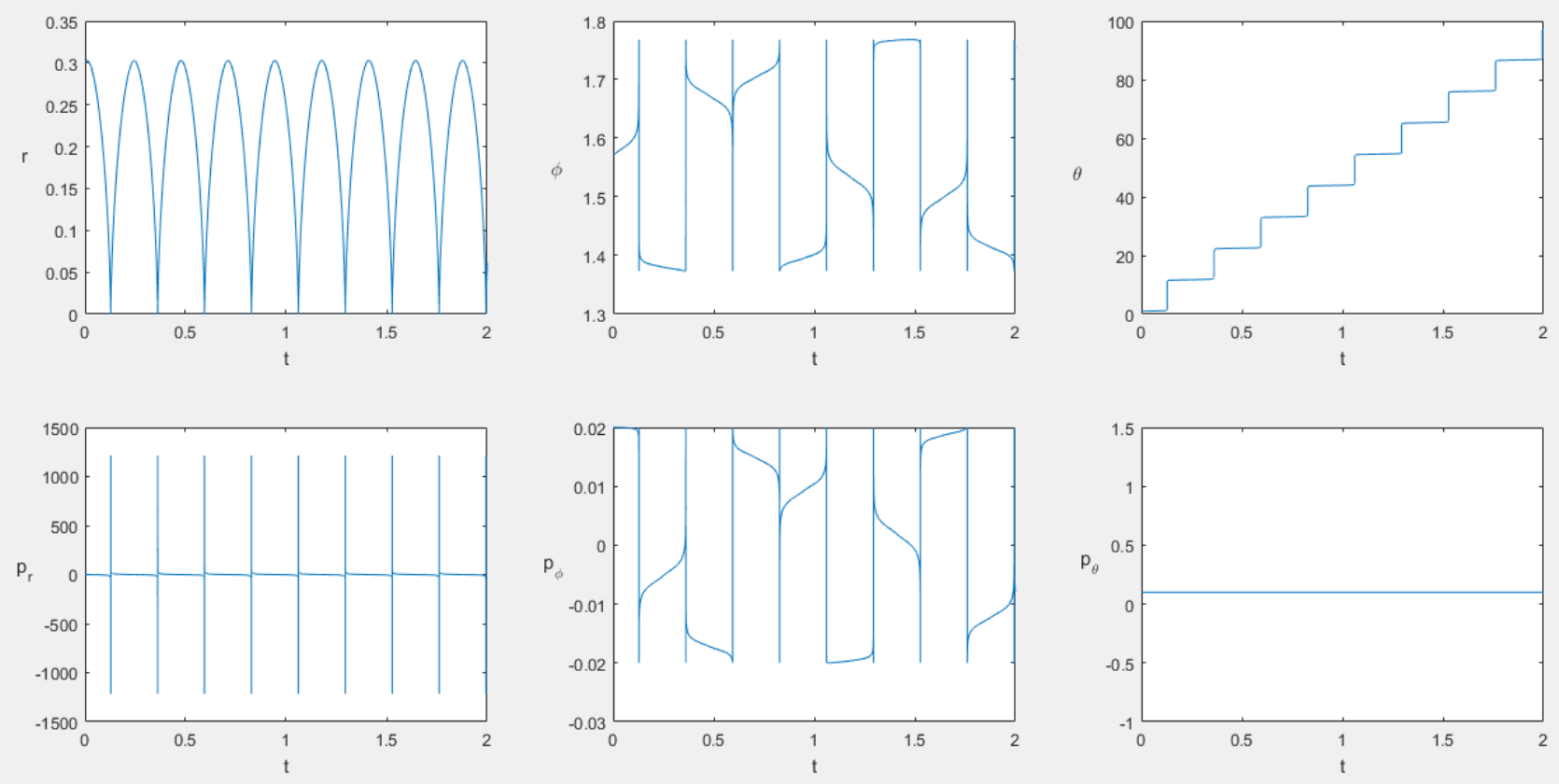

Figure 4.2.17: $\alpha=1.75, \quad \mathcal{H}_{3, K}\left(0.3, \frac{\pi}{2}, 1,0.4,0.02,0.1\right)=-8.5$

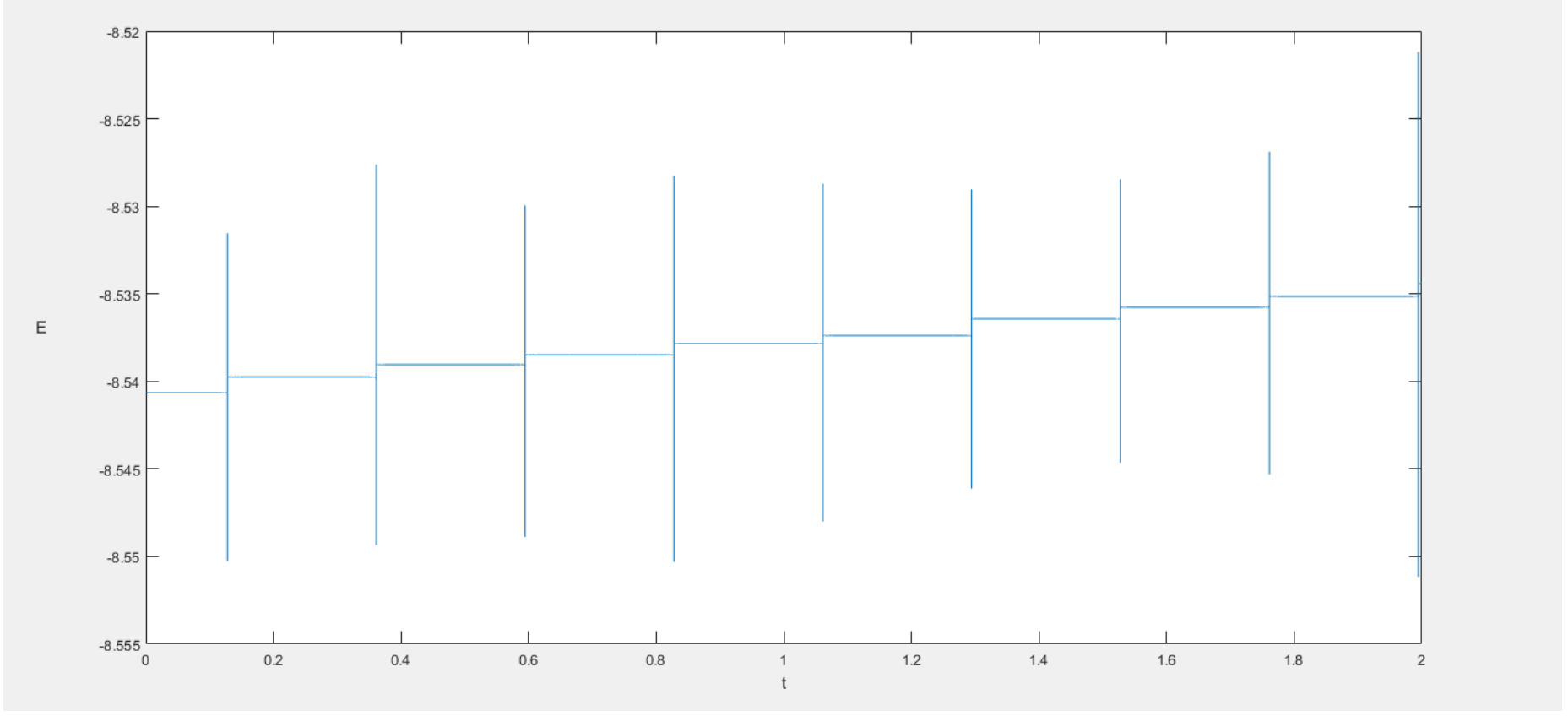

Figure 4.2.18 

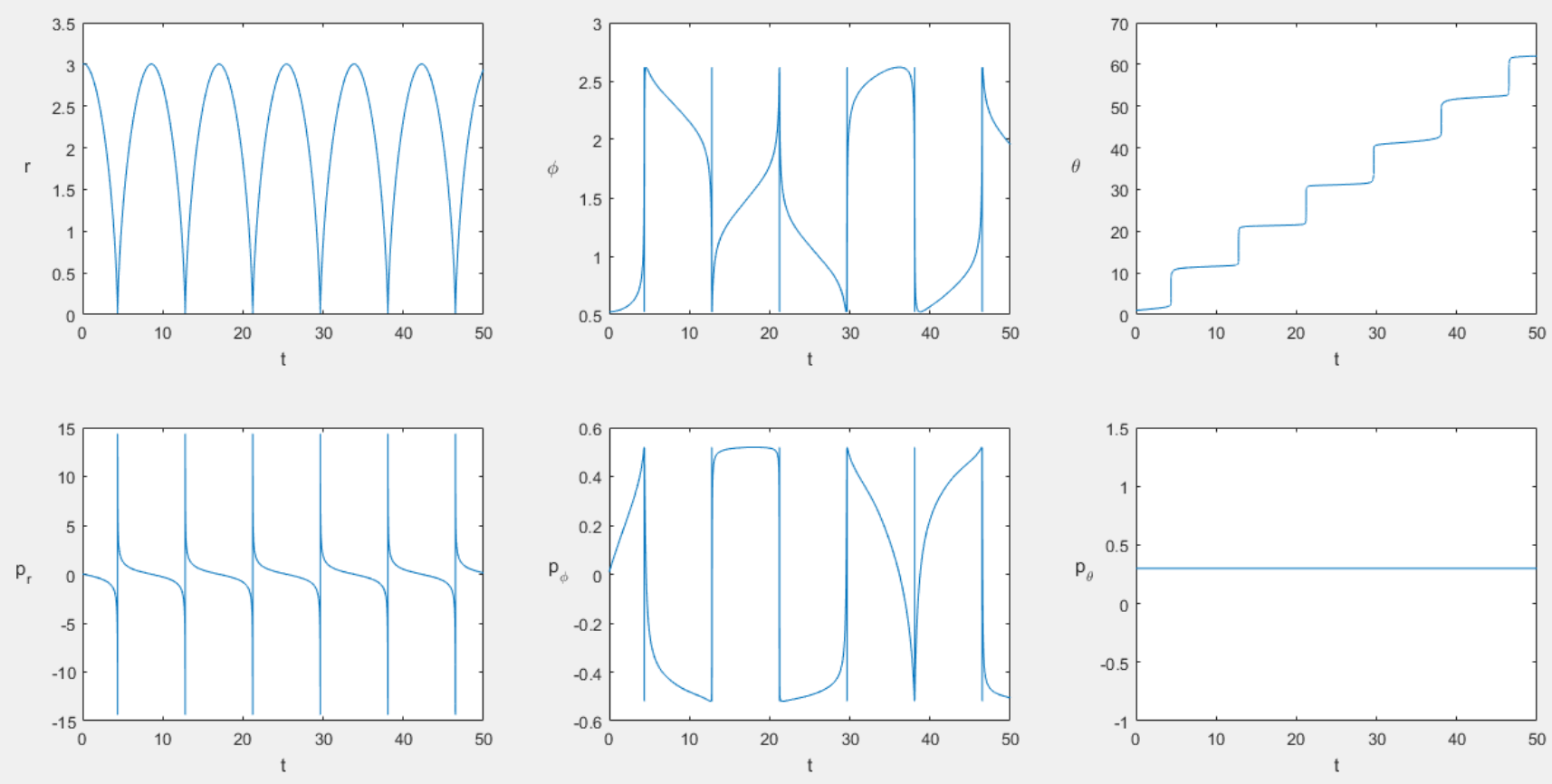

Figure 4.2.19: $\alpha=1.75, \quad \mathcal{H}_{3, K}\left(3, \frac{\pi}{6}, 1,0.03,0.01,0.3\right)=-0.4558$

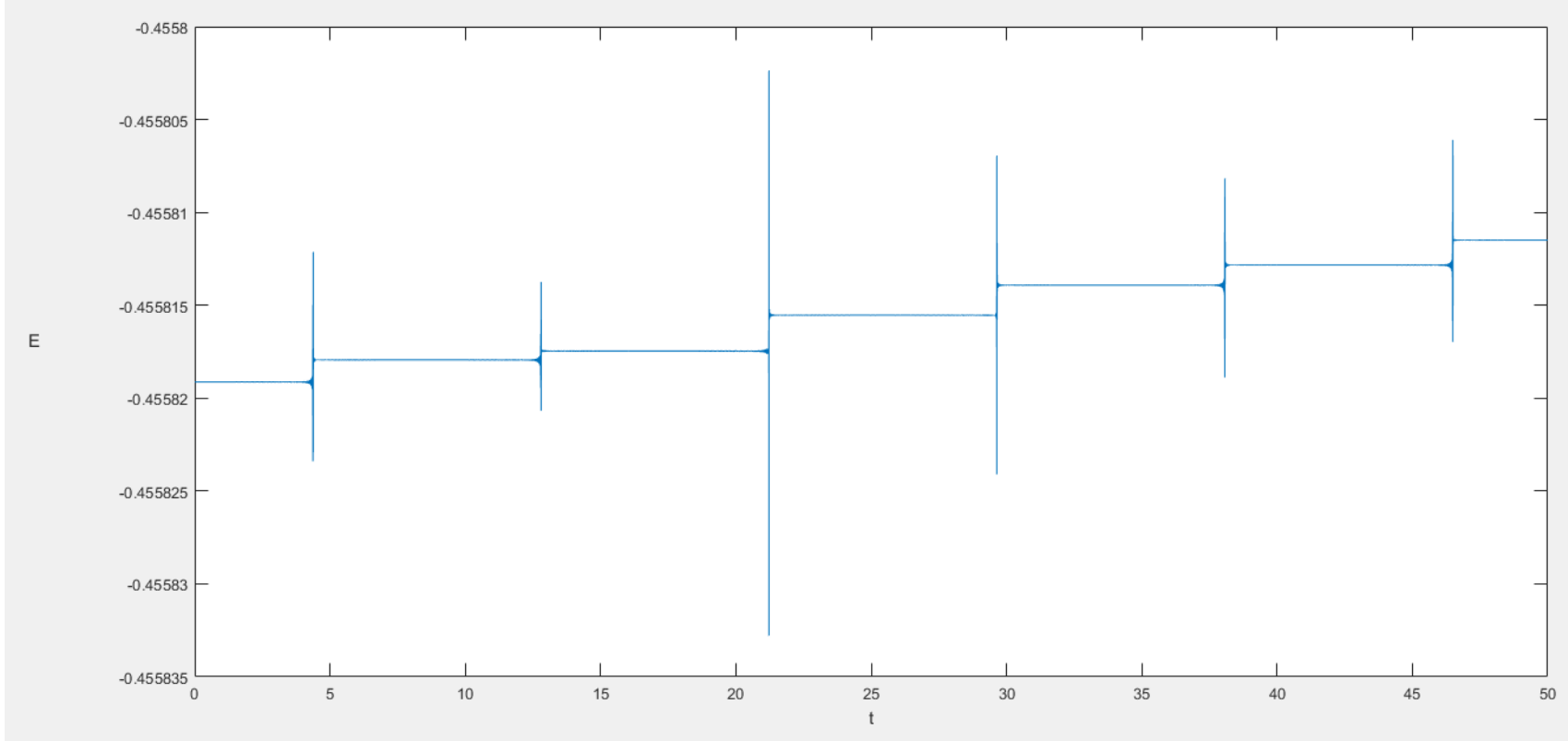

Figure 4.2 .20 

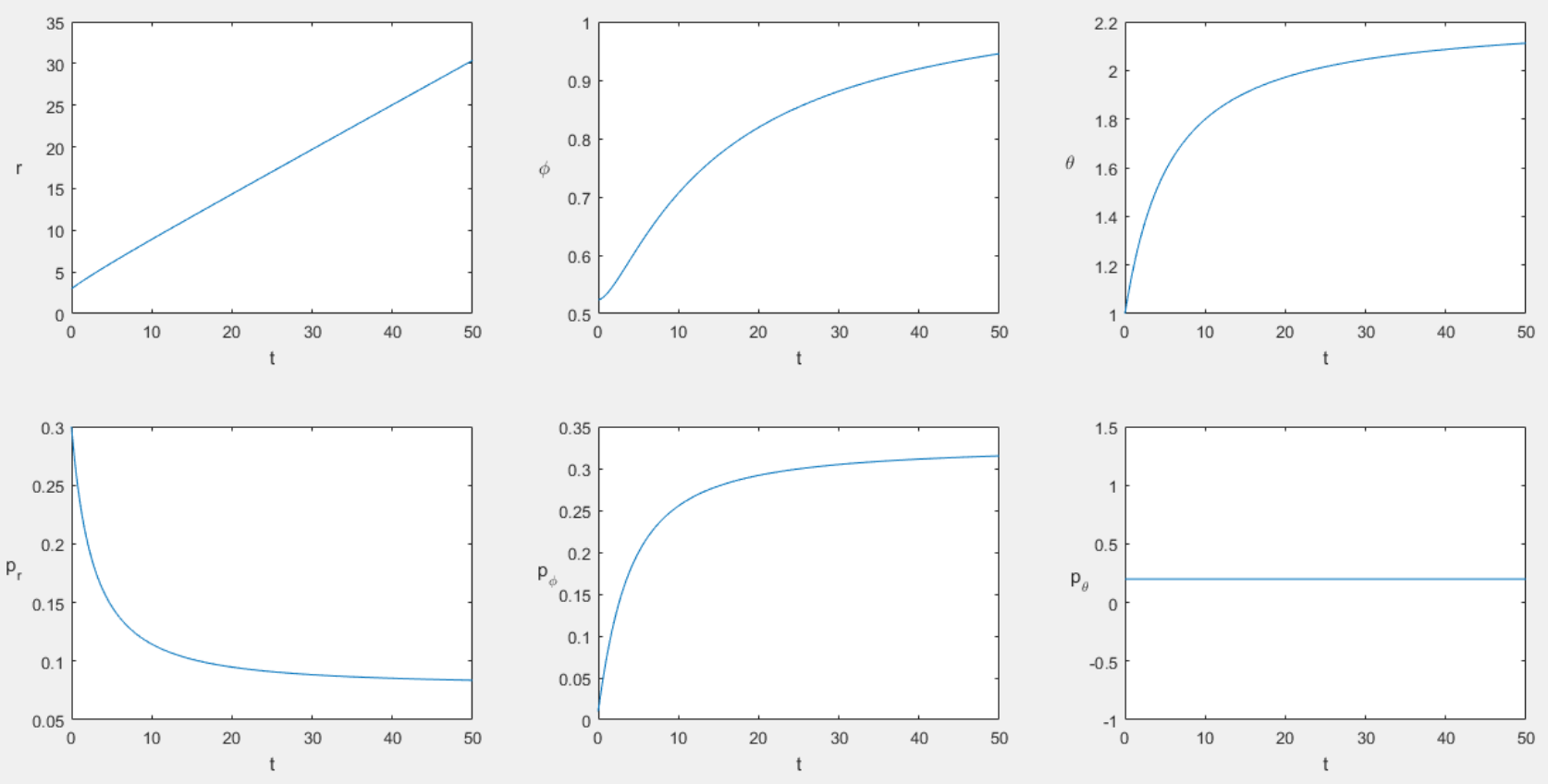

Figure 4.2.21: $\alpha=1.25, \quad \mathcal{H}_{3, K}\left(3, \frac{\pi}{6}, 1,0.3,0.01,0.2\right)=0.03369962$

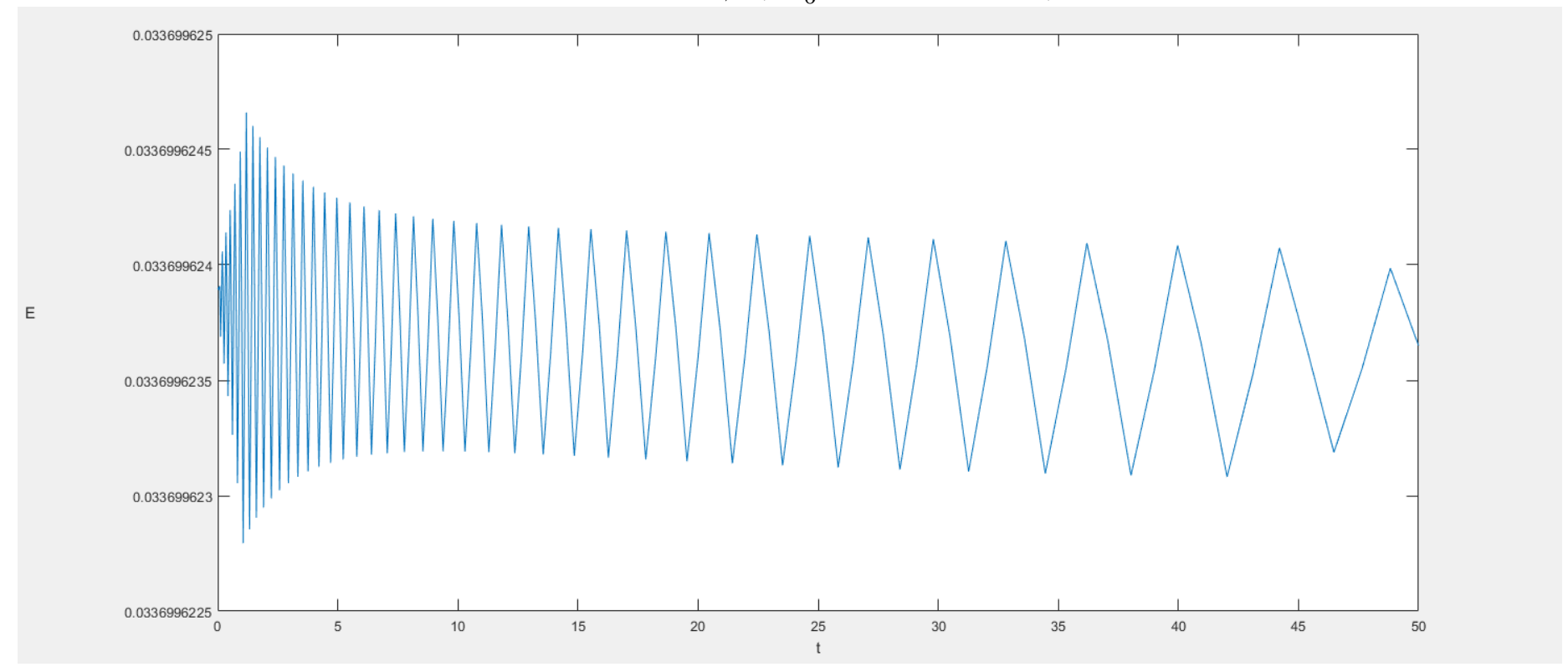

Figure 4.2 .22 

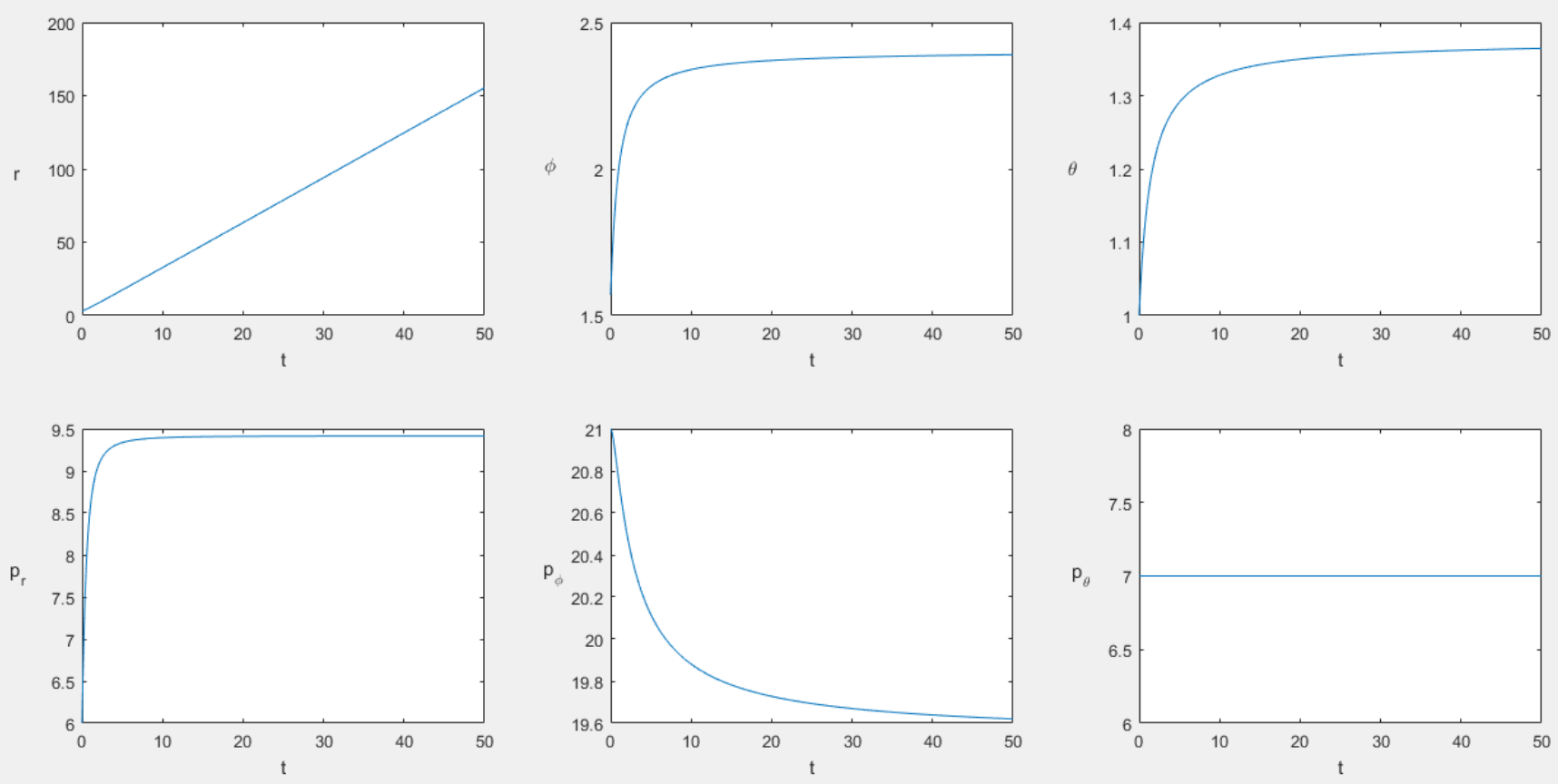

Figure 4.2.23: $\alpha=1.5, \quad \mathcal{H}_{3, K}\left(3, \frac{\pi}{2}, 1,6,21,7\right)=19.2635$

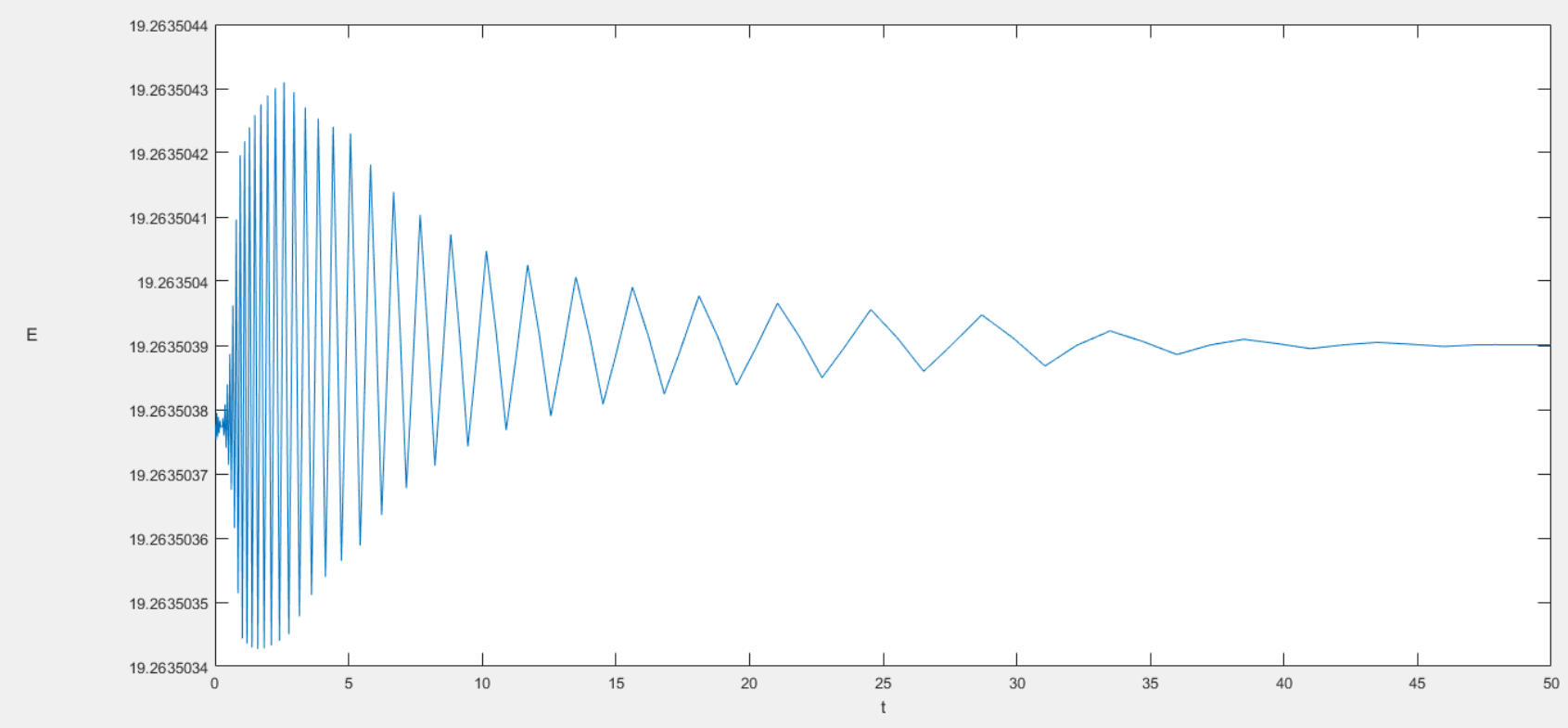

Figure 4.2.24 
As with the two-dimensional case we seem to have two distinct cases. More positive energies again seems to suggest unbounded motion where the more negative energies suggest oscillatory motion. We adopt the same approach as before to not repeat any analysis that would be too similar to what we have already seen. Comparing the above figures to the two-dimensional case we see that $r, p_{r}, \theta, p_{\theta}$ and $E$ in the three dimensions all follow similar behaviours to $r, p_{r}, \vartheta, p_{\vartheta}$ and $E$ in two dimensions. Hence we refer to Example 4.2.2 for their analysis.

To move on to a new coordinate $\phi$, we see that for the oscilltory case it undergoes large change as $r$ reaches it points of changing direction. The coordinate $p_{\phi}$ supports this as it too changes the most when $\phi$ does. This is to be expected as physically, $p_{\phi}$ being the conjugate momentum depends on the rate of change of $\phi$. We also notice $\phi$ seems to be getting smaller as $E$ becomes more negative. Therefore in this oscilatory case, as well as having the usual flower with petals trace as before, this path must now be oscillating orthogonal to the path of travel. It seems as the energy decreases this oscillation becomes smaller. However when the radius is changed by a large scale so does the size of the oscillation.

To move on to the unbounded case we see that for small positive energies both $\phi$ and $p_{\phi}$ seem to increase. This shows that maybe for such energies the spiral shape that is formed is rising upwards while accelarating before easing off towards the end. For large values of energy a converse of sorts is happening where the spiral is still rising with the increase of $\phi$ however this time $p_{\phi}$ decreases suggesting that the spiral decelerates instead.

\subsection{Relativistic Dynamics}

In classical mechanics, one can obtain a Lagrangian formulation for the relativistic dynamics of systems with one particle. This is disussed at [26, Chapter 7.9]. Here we find that the Lagrangian takes the form

$$
\mathcal{L}(q, \dot{q})=-m c^{2} \sqrt{1-\frac{\|\dot{q}\|^{2}}{c^{2}}}-V(q)
$$

where $m$ denotes the mass of the particle. The corresponding Hamiltonian is then found to be

$$
\mathcal{H}(q, p)=\sqrt{p^{2} c^{2}+m^{2} c^{4}}+V(q)
$$

where $c$ is some positive constant, i.e. the velocity of light (see also [26, Chapter 8.4]).

In reference to (4.3.2) the Hamilton function we will consider is $\mathcal{H}_{R} \in C^{1}\left(\mathbb{R}^{2 n}\right)$

$$
\mathcal{H}_{R}(q, p)=\sqrt{m^{2}+\|p\|^{2}}-m+V(q)
$$


where $V \in C^{1}\left(\mathbb{R}^{n}\right)$ and $m>0$. We have assumed $c=1$ for simplicity. Comparing $(4.3 .3)$ to

$$
\mathcal{H}(q, p)=\psi(p)+V(q), \quad \psi \in C N\left(\mathbb{R}^{n}\right) \cap C^{1}\left(\mathbb{R}^{n}\right)
$$

we identify

$$
\psi(p):=\sqrt{m^{2}+\|p\|^{2}}-m .
$$

The function $\psi$ as defined at (4.3.5) is indeed continuous and when comparing back to Example 2.4.B of Chapter 2, certainly negative definite. One may also deduce that $\psi$ is convex for $n=1,2,3$. We choose to add $-m$ at (4.3.5) since we want to keep the property $\psi(0)=0$, a necessity for the metric $d_{\psi}:=\psi^{\frac{1}{2}}$ from Chapter 2 .

A problem appears however with coercivity. It holds that

$$
\lim _{\|p\| \rightarrow \infty} \frac{\sqrt{m^{2}+\|p\|^{2}}-m}{\|p\|}=1
$$

hence following Proposition 1.13 we cannot currently guarantee that its conjugate function is finite. If we want to look at the corresponding Lagrange function as before then this is an issue we cannot ignore. The conjugate function to (4.3.5) follows as

$$
\psi^{*}(\eta)=m\left(1-\sqrt{1-\|\eta\|^{2}}\right) .
$$

With $\psi$ being rotational invariant its conjugate at (4.3.6) may be found using Theorem 1.16. As a convex function, for (4.3.6) to be proper we need $\|\eta\| \leq 1$, i.e. the domain of $\psi^{*}$ to be $B_{1}(0)$. Through this, we define $\psi^{*}$ by $\psi^{*}: \mathbb{R}^{n} \longrightarrow \mathbb{R} \cup\{\infty\}$ such that

$$
\psi^{*}(\eta):= \begin{cases}m\left(1-\sqrt{1-\|\eta\|^{2}}\right), & \text { if } \eta \in B_{1}(0), \\ +\infty, & \text { if } \eta \notin B_{1}(0) .\end{cases}
$$

The corresponding Lagrange function to (4.3.3) must now follow as

$$
\mathcal{L}_{R}(q, \eta)=m\left(1-\sqrt{1-\|\eta\|^{2}}\right)-V(q)
$$

where we have used (4.3.6). As before $\eta=\dot{q}$. We consider $\mathcal{L}_{R}$ as a function on $\mathbb{R}^{n} \times B_{1}(0)$, and note that $\mathcal{L}_{R}(., \eta) \in C^{1}\left(\mathbb{R}^{n}\right)$ and $\mathcal{L}_{R}(q,.) \in C^{2}\left(B_{1}(0)\right)$. Notice this compares well with the Lagrangian (4.3.1) from the relativistic dynamics.

Comparing back to Section 3.3 where we discussed conservation laws associated with our dynamics a problem now appears. We recall that given a Hamilton function with kinetic term $\psi \in C^{1}\left(\mathbb{R}^{n}\right)$, we identified the corresponding Lagrange function as having a dual kinetic term $\psi^{*} \in C^{2}\left(\mathbb{R}^{n}\right)$. However here in the case of the relativistic dynamics, for a kinetic term $\psi \in C^{1}\left(\mathbb{R}^{n}\right)$ we find that its dual kinetic term in the Lagrange function (4.3.8) must be an element of $C^{2}\left(B_{1}(0)\right)$. Hence the duality in this case breaks down. 
As before we will take the conjugate of the kinetic term to be an example for the potential. In this case it follows that $V \in C^{1}\left(B_{1}(0)\right)$ such that

$$
V(q)=\psi^{*}(q)=m\left(1-\sqrt{1-\|q\|^{2}}\right) .
$$

It holds that $m \leq \sqrt{m^{2}+\|p\|^{2}}$ and $m \sqrt{1-\|q\|^{2}} \leq m$ whenever $\|q\|<1$. Hence the kinetic term at (4.3.5) is more than or equal to the above potential. Therefore in this particular example we know that the conserved value of the Hamilton function $E$ is always non-negative, as was the case with examples associated with the Harmonic Oscillator.

Next come the examples. As before we will investigate the dynamics of the Hamilton and Lagrange functions (4.3.3) and (4.3.8) in one, two and three dimensions. The full calculations for the one and two-dimensional examples can be fully illustrated by analogously following the examples at Appendix B. Similarly following Example 6.2.3 from Section 6.2 covers the three-dimensional case. One will see that many of the solutions are determined by integrals with inexplicit forms. As before we include graphics to help us learn more about the solutions. Unlike the previous examples we do not have a fractional power $\alpha$ in play now but a new parameter $m>0$. Hence different choices of $m$ will be considered in these graphics.

Example 4.3.1. We would like to study the dynamics associated with the function $\mathcal{H}_{1, R} \in C^{1}((-1,1) \times \mathbb{R} \backslash\{0\})$ such that

$$
\mathcal{H}_{1, R}(q, p)=\sqrt{m^{2}+p^{2}}-m \sqrt{1-q^{2}}, \quad m>0 .
$$

The associated Hamilton's equations follow as

$$
\left\{\begin{array}{l}
\dot{q}=\frac{p}{\sqrt{m^{2}+p^{2}}}, \\
\dot{p}=-\frac{m q}{\sqrt{1-q^{2}}} .
\end{array}\right.
$$

As with previous examples we set initial conditions to employ when solving the above system. The conditions once again follow as $q\left(t_{0}\right)=q_{0}$ and $p\left(t_{0}\right)=p_{0}$. They determine the conserved value $E$ of (4.3.10) i.e. $\mathcal{H}_{1, R}(q(t), p(t))=\mathcal{H}_{1, R}\left(q_{0}, p_{0}\right)=E$. Through this Hamilton's equations can be found to be

$$
\left\{\begin{array}{l}
\dot{q}= \pm \frac{\sqrt{\left(E+m \sqrt{1-q^{2}}\right)^{2}-m^{2}}}{E+m \sqrt{1-q^{2}}} \\
\dot{p}= \pm \frac{m \sqrt{m^{2}-\left(\sqrt{m^{2}+p^{2}}-E\right)^{2}}}{\sqrt{m^{2}+p^{2}}-E}
\end{array}\right.
$$


By separation of variables the coordinates satisfy

$$
\pm \int \frac{E+m \sqrt{1-q^{2}}}{\sqrt{\left(E+m \sqrt{1-q^{2}}\right)^{2}-m^{2}}} \mathrm{~d} q=A_{1}+t
$$

and

$$
\pm \frac{1}{m} \int \frac{\sqrt{m^{2}+p^{2}}-E}{\sqrt{m^{2}-\left(\sqrt{m^{2}+p^{2}}-E\right)^{2}}} \mathrm{~d} p=A_{2}+t
$$

where $A_{1}$ and $A_{2}$ are constants of integration. Assuming the above integrations are possible we can determine $q$ and $p$ in terms of $t$ and the constants of integration. However as before these constants may be eliminated by employing the initial conditions. This then finds $A_{1}$ and $A_{2}$ in terms of $E, q_{0}, p_{0}$ and $t_{0}$. Hence $q$ and $p$ may then be found in terms of these constants as well as $t$.

The corresponding Lagrange function is

$$
\mathcal{L}_{1, R}(q, \eta)=-m \sqrt{1-\eta^{2}}+m \sqrt{1-q^{2}}
$$

where for $q$ and $\eta$ fixed, $\mathcal{L}_{1, R}(., \eta) \in C^{1}((-1,1))$ and $\mathcal{L}_{1, R}(q,.) \in C^{2}((-1,1))$. Lagrange's equation follows as

$$
\ddot{q}=\frac{-q\left(1-\dot{q}^{2}\right)^{\frac{3}{2}}}{\sqrt{1-q^{2}}} .
$$

We know that E may also be given by

$$
\frac{\partial \mathcal{L}_{1, R}}{\partial \eta} \eta-\mathcal{L}_{1, R}=E
$$

or

$$
\dot{q}= \pm \frac{\sqrt{\left(E+m \sqrt{1-q^{2}}\right)^{2}-m^{2}}}{E+m \sqrt{1-q^{2}}} .
$$

This coincides with the first equation of the system (4.3.12). Hence, solving for $q$ follows as before. Once having obtained the complete solution for $q$, the generalised velocity $\dot{q}$ may be determined using (4.3.18).

As with some previous examples, there are some conditions we must check so that everything is well-defined:

(i) $E+m \sqrt{1-q^{2}} \neq 0$, 
(ii) $\sqrt{m^{2}+p^{2}}-E \neq 0$

(iii) $\left(E+m \sqrt{1-q^{2}}\right)^{2}-m^{2}>0$,

(iv) $m^{2}-\left(\sqrt{m^{2}+p^{2}}-E\right)^{2}>0$.

Consider conditions (i) and (ii). We begin by recollecting that $m>0$ and $q \in$ $(-1,1)$. Following a similar method to Example 4.1.2 we take $E$ as its form of the Hamilton function (4.3.10). Substituting (4.3.10) into the first and second conditions above we have

$$
\sqrt{m^{2}+p^{2}} \neq 0 \text { and } m \sqrt{1-q^{2}} \neq 0
$$

respectively. Using that $m>0$ and $q \in(-1,1)$ equation (4.3.19) implies that conditions (i) and (ii) do indeed hold.

For the third and fourth conditions we now use that $p \neq 0$. Following a similar method again where we use (4.3.10) as E, conditions (iii) and (iv) give

$$
p^{2}>0 \quad \text { and } m^{2} q^{2}>0
$$

respectively. The above are of course true whenever $p \neq 0$ and if additionally $q \neq 0$.

For selected values of $\alpha$ below are some examples of the phase diagrams for (4.3.11) constructed using Mathematica. The horizontal axis represents the coordinate $q$ where the vertical axis corresponds to $p$. 


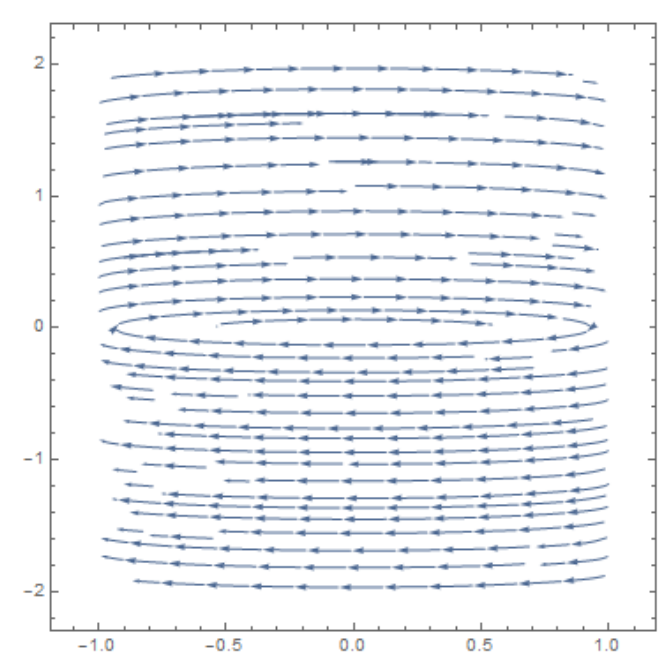

Figure 4.3.1: $m=0.1$

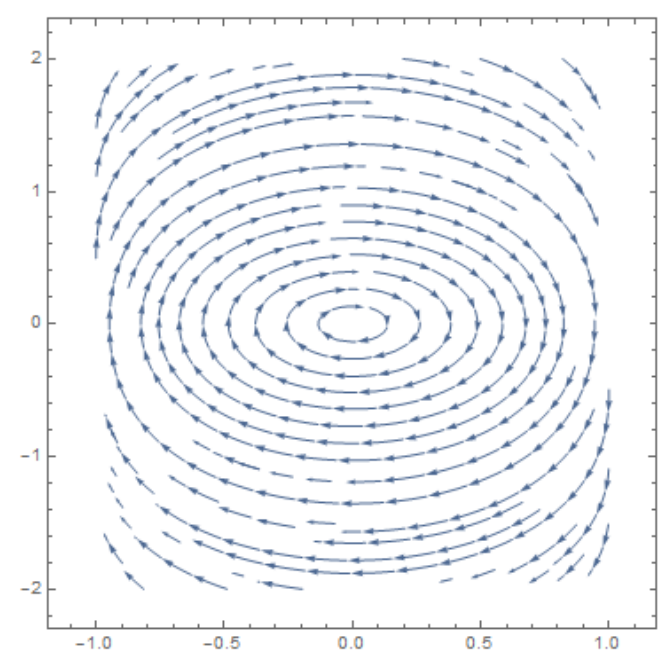

Figure 4.3.3: $m=1$

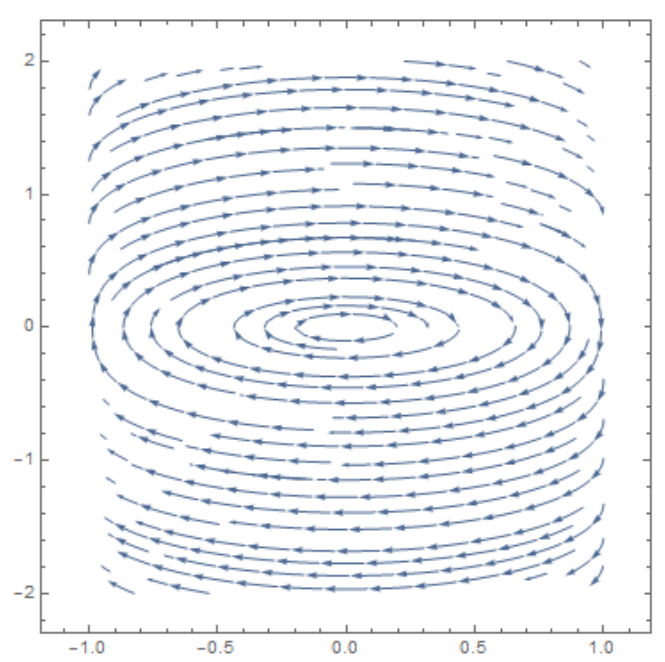

Figure 4.3.2: $m=0.5$

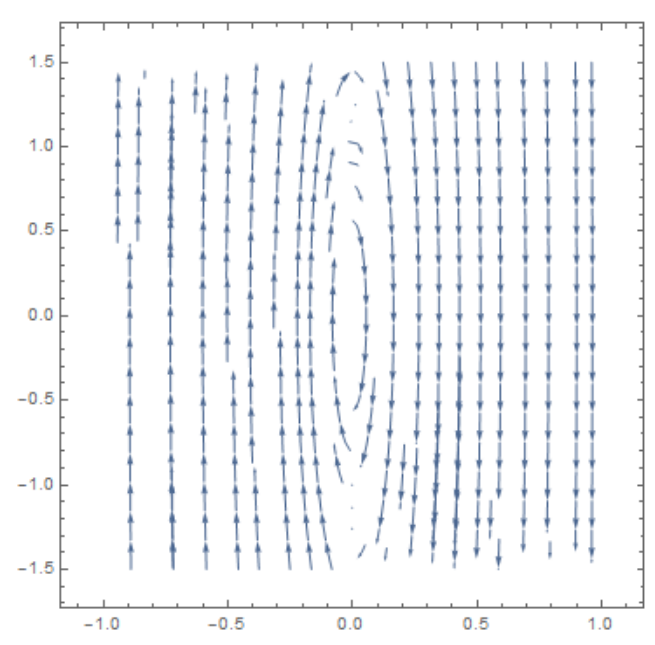

Figure 4.3.4: $m=10$

Observing the above figures we can see that the solutions have a symmetric property when reflected over the lines $q=-p, p=0$ and $q=0$. In other words given the solutions for when $q \geq 0$ and $p \geq 0$, we can solve the cases for $q<0$ and $p<0$; $q \geq 0$ and $p<0 ; q<0$ and $p \geq 0$ by reflecting over $q=-p, p=0$ and $q=0$ respectively in the phase space and taking the appropiate sign. Additionally, observing the figures above we see that they suggest a periodic property in the solutions, in particular with $m=1$ demonstrating behaviour quite close to what we expect from the Harmonic Oscillator in classical mechanics. 
Example 4.3.2. We want to study the dynamics associated with $\mathcal{H} \in C^{1}\left(\mathbb{R}^{4}\right)$ such that

$$
\mathcal{H}(q, p)=\sqrt{m^{2}+\|p\|^{2}}-m+V(q) .
$$

The associated Hamilton's equations follow as

$$
\left\{\begin{array}{l}
\dot{q}_{j}=\frac{p_{j}}{\sqrt{m^{2}+\|p\|^{2}}}, \quad j=1,2 . \\
\dot{p}_{j}=-V_{q_{j}}(q),
\end{array}\right.
$$

As before having more conserved quantites facilitates the solving. Hence we transform to polar coordinates so that the function (4.3.20) is equivalent to $\mathcal{H}_{2, R} \in$ $C^{1}((0, \infty) \times[0,2 \pi) \times \mathbb{R} \backslash\{0\} \times \mathbb{R})$ such that

$$
\mathcal{H}_{2, R}\left(r, \vartheta, p_{r}, p_{\vartheta}\right)=\sqrt{m^{2}+p_{r}^{2}+\frac{p_{\vartheta}^{2}}{r^{2}}}-m+V(r) .
$$

Hamilton's equations now follow as

$$
\begin{gathered}
\dot{r}=\frac{p_{r}}{\sqrt{m^{2}+p_{r}^{2}+\frac{p_{\vartheta}^{2}}{r^{2}}}}, \\
\dot{\vartheta}=\frac{p_{\vartheta}}{r^{2} \sqrt{m^{2}+p_{r}^{2}+\frac{p_{\vartheta}^{2}}{r^{2}}}}, \\
\dot{p}_{r}=\frac{p_{\vartheta}^{2}}{r^{3} \sqrt{m^{2}+p_{r}^{2}+\frac{p_{\vartheta}^{2}}{r^{2}}}}-V_{r}(r)
\end{gathered}
$$

and

$$
\dot{p}_{\vartheta}=0
$$

implying that $p_{\vartheta}(t)=\kappa$ for all $t, \kappa$ being some constant. Therefore with $\vartheta$ being a cyclic coordinate of (4.3.22), the claim of Theorem 3.3.5 is satisfied.

The initial conditions determine the conserved value $E$ of the Hamilton function, i.e. $\mathcal{H}_{2, R}\left(r(t), \vartheta(t), p_{r}(t), p_{\vartheta}(t)\right)=\mathcal{H}_{2, R}\left(r^{0}, \vartheta^{0}, p_{r}^{0}, p_{\vartheta}^{0}\right)=E$. Through this we can deduce that $p_{r}$ is

$$
p_{r}= \pm \sqrt{(E+m-V(r))^{2}-m^{2}-\frac{\kappa}{r^{2}}}
$$

such that

$$
\dot{r}= \pm \frac{\sqrt{(E+m-V(r))^{2}-m^{2}-\frac{\kappa}{r^{2}}}}{E+m-V(r)}
$$

and

$$
\dot{\vartheta}=\frac{\kappa}{r^{2}(E+m-V(r))} .
$$


Then $r$ is found to satisfy

$$
\gamma+t= \pm \int \frac{r(E+m-V(r))}{\sqrt{r^{2}\left((E+m-V(r))^{2}-m^{2}\right)-\kappa^{2}}} \mathrm{~d} r
$$

where $\gamma$ is a constant obtained through integration. Assuming the integration to be possible $r$ may be determined in terms of $t$ only. The constant of integration may be eliminated as before through the incorporation of initial conditions.

We may then use this solution for $r$ with (4.3.28) to obtain a first-order differential equation of only $\vartheta$. This equation may then be integrated to find $\vartheta$ introducing a further constant of integration. This constant may then be eliminated as before using initial conditions.

The corresponding Lagrange function is

$$
\mathcal{L}(q, \eta)=m\left(1-\sqrt{1-\|\eta\|^{2}}\right)-V(q)
$$

where $\mathcal{L}(., \eta) \in C^{1}\left(\mathbb{R}^{n}\right)$ and $\mathcal{L}(q,.) \in C^{2}\left(B_{1}(0)\right)$. Lagrange's equations follow as

$$
\frac{m \dot{\eta}_{j}}{\sqrt{1-\|\eta\|^{2}}}+\frac{m \eta_{j}}{\left(1-\|\eta\|^{2}\right)^{\frac{3}{2}}} \sum_{l=1}^{n} \eta_{l} \dot{\eta}_{l}=-V_{q_{j}}(q), \quad j=1, \ldots, n .
$$

Transforming the function at (4.3.30) to polar coordinates gives a new function

$$
\mathcal{L}_{2, R}(r, \vartheta, \dot{r}, \dot{\vartheta})=m\left(1-\sqrt{1-\dot{r}^{2}-r^{2} \dot{\vartheta}^{2}}\right)-V(r)
$$

where $\mathcal{L}_{2, R}(., ., \dot{r}, \dot{\vartheta}) \in C^{1}((0, \infty) \times[0,2 \pi))$ and $\mathcal{L}_{2, R}(r, \vartheta, .,.) \in C^{2}\left(\mathbb{R}^{2}\right)$. Lagrange's equations follow as

$$
\frac{\mathrm{d}}{\mathrm{d} t}\left(\frac{m \dot{r}}{\sqrt{1-\dot{r}^{2}-r^{2} \dot{\vartheta}^{2}}}\right)=\frac{m r \dot{\vartheta}^{2}}{\sqrt{1-\dot{r}^{2}-r^{2} \dot{\vartheta}^{2}}}-V_{r}(r)
$$

and

$$
\frac{\mathrm{d}}{\mathrm{d} t}\left(\frac{m r^{2} \dot{\vartheta}}{\sqrt{1-\dot{r}^{2}-r^{2} \dot{\vartheta}^{2}}}\right)=0
$$

implying that $p_{\vartheta}(t)=\kappa$ for all $t, \kappa$ being some constant. Therefore the coordinate $p_{\vartheta}$ corresponding to the cyclic coordinate $\vartheta$ is conserved as expected from Theorem 3.3.6.

Recollecting from Theorem 3.3.2 that $E$ is given by

$$
\frac{\partial \mathcal{L}_{2, R}}{\partial \dot{r}} \dot{r}+\frac{\partial \mathcal{L}_{2, R}}{\partial \dot{\vartheta}} \dot{\vartheta}-\mathcal{L}_{2, R}=E
$$


we have

$$
\dot{r}=\frac{\sqrt{(E+m-V(r))^{2}-m^{2}-\frac{\kappa}{r^{2}}}}{E+m-V(r)}
$$

and

$$
\dot{\vartheta}=\frac{\kappa}{r^{2}(E+m-V(r))} .
$$

Comparing (4.3.34) and (4.3.35) to (4.3.27) and (4.3.28) we see that the solutions can now be found as they were with the Hamilton function. Having done this, equations (4.3.34) and (4.3.35) can then be used to solve for $\dot{r}$ and $\dot{\vartheta}$ respectively.

A concern is the term $1-\dot{r}^{2}-r^{2} \dot{\vartheta}^{2}$ not being strictly positive. However, we should recall that $\dot{r}^{2}+r^{2} \dot{\vartheta}^{2}$ is $\|\eta\|^{2}$ but in the form of polar coordinates. Hence by taking the condition $\|\eta\|<1$, we have this singularity avoided.

As with other examples there are some conditions we need to impose such that the results are well-defined:

(i) $E+m-V(r) \neq 0$,

(ii) $r^{2}\left((E+m-V(r))^{2}-m^{2}\right)-\kappa^{2}>0$.

We deal with the above as we did in Examples 4.1.2. Through this it is sufficent to have $p_{r} \neq 0$ for the above to hold.

We have a solution (4.3.29) with a form that does not tell us much about its behaviour. Hence with the help of Matlab below are figures presenting plots approximating the evolution of the coordinates satisfying Hamilton's equations, as well as plots of E. In each case it is specified in the captions the specific value of $\alpha$ and the corresponding initial values that were taken. To be able to construct the figures we need to choose a specific potential. Following (4.3.9) we take this potential to be the conjugate of the kinetic term i.e.

$$
V(r)=m\left(1-\sqrt{1-r^{2}}\right), \quad r \in(0,1) .
$$



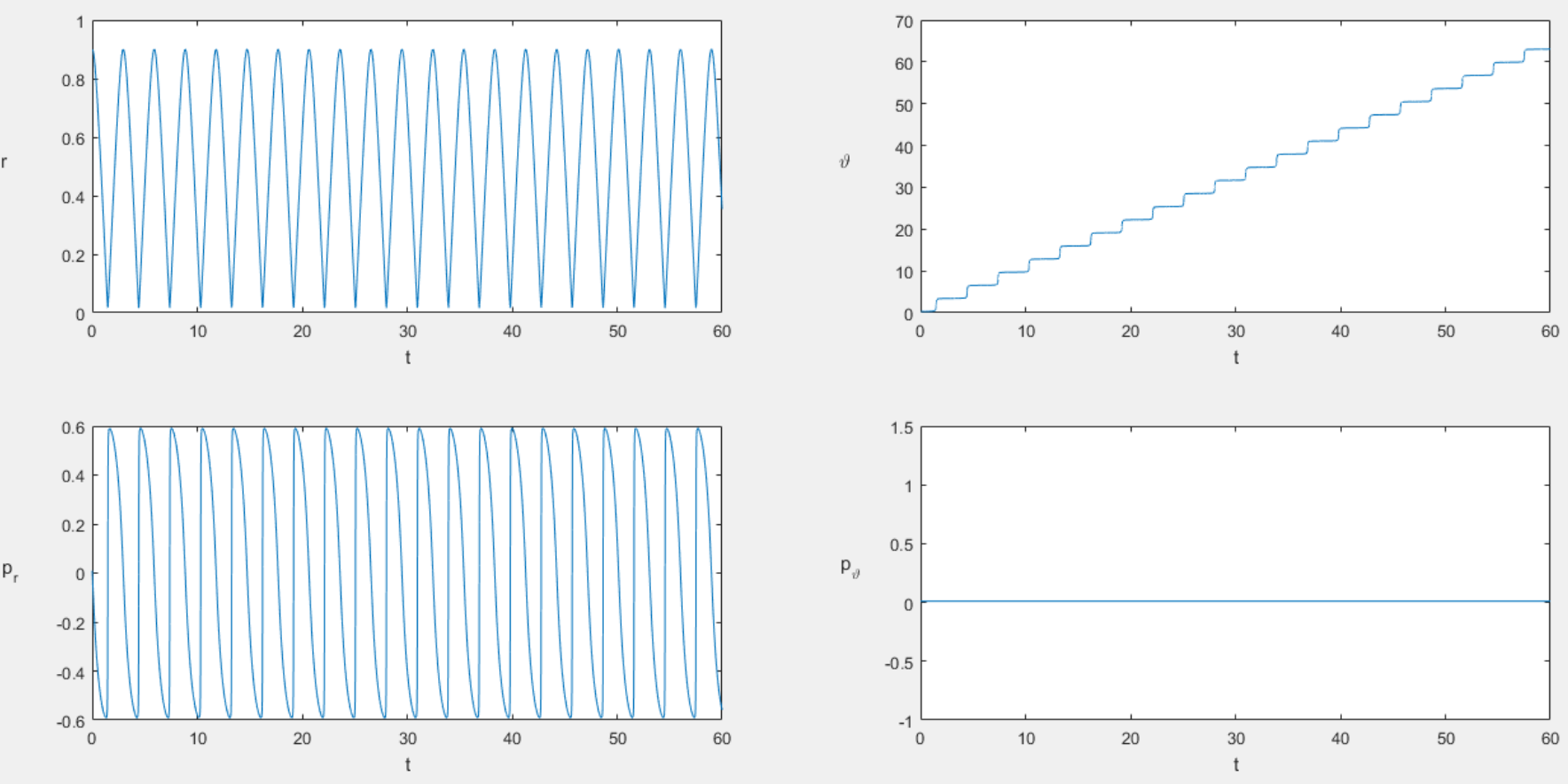

Figure 4.3.5: $m=\frac{1}{2}, \quad \mathcal{H}_{2, R}(0.9,0.3,0.01,0.01)=0.282278$

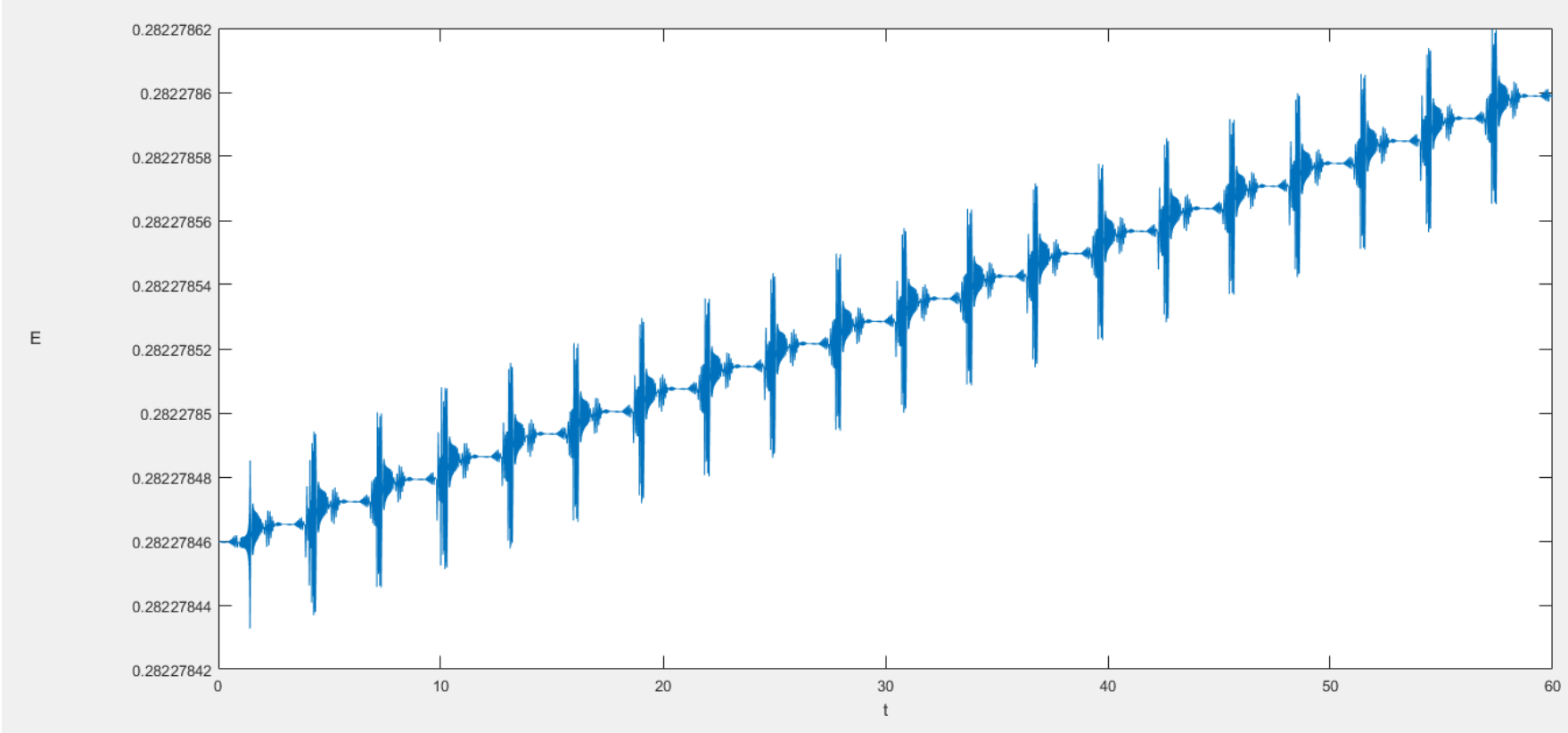

Figure 4.3.6 

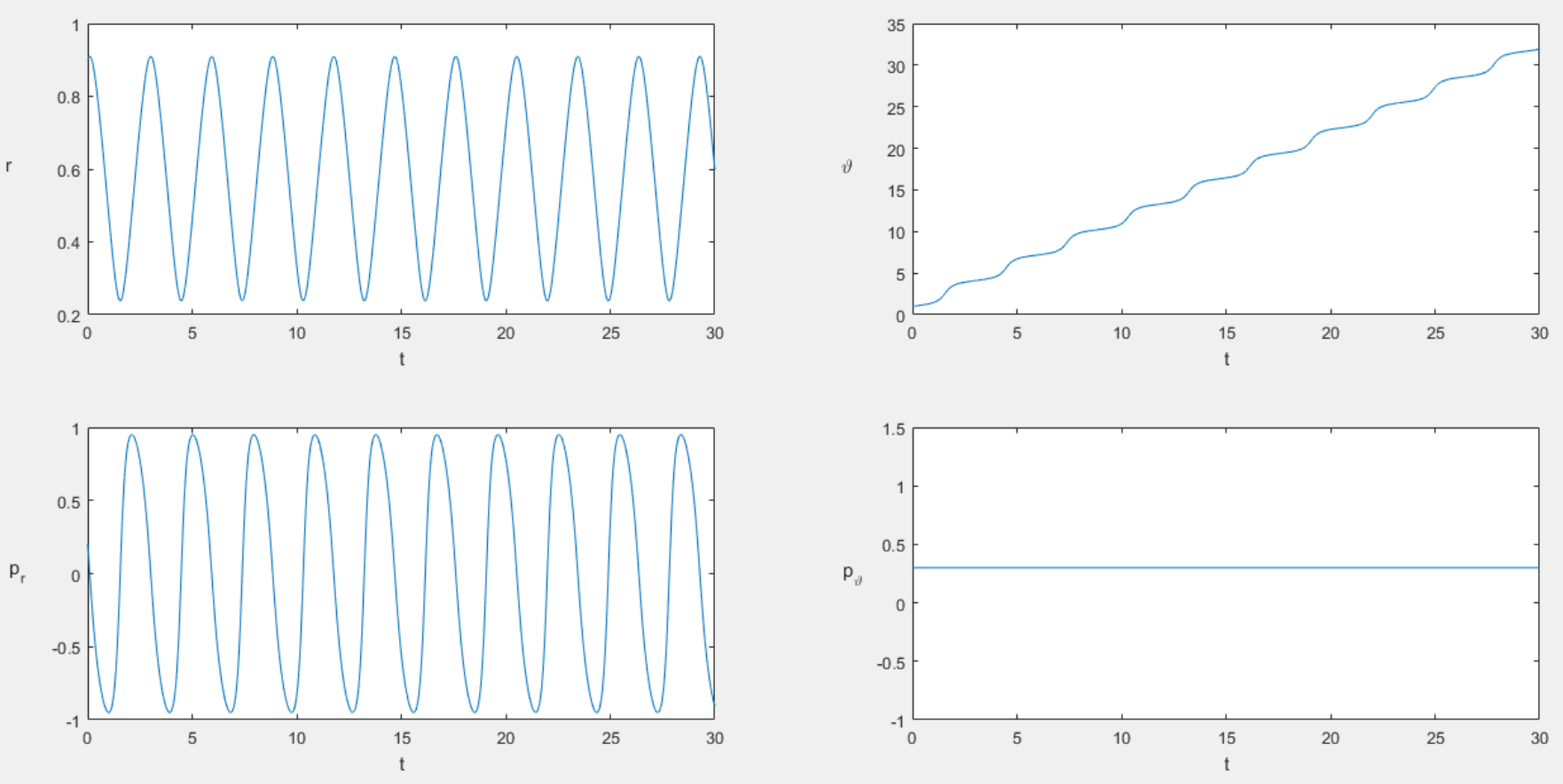

Figure 4.3.7: $m=1, \quad \mathcal{H}_{2, R}(0.9,1,0.2,0.3)=0.637008$

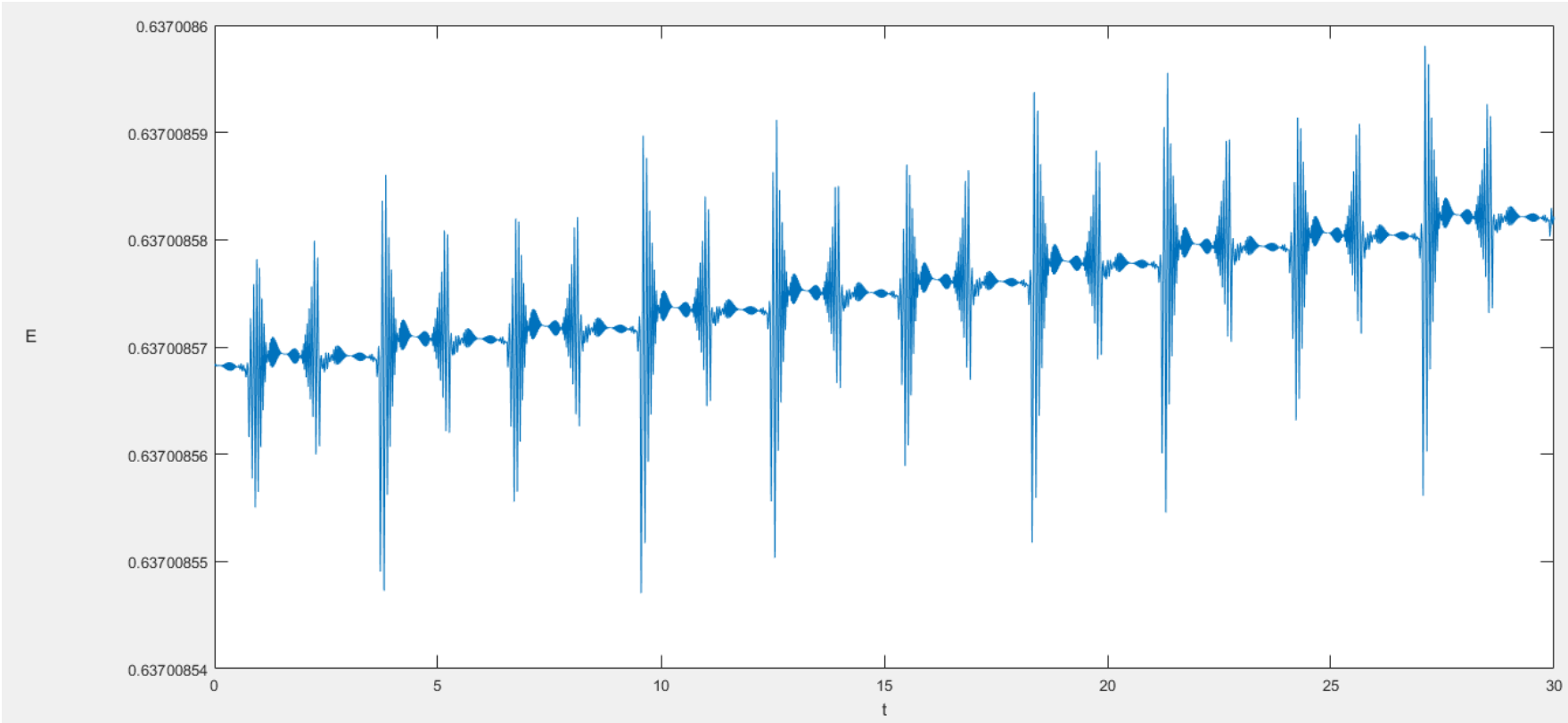

Figure 4.3.8 

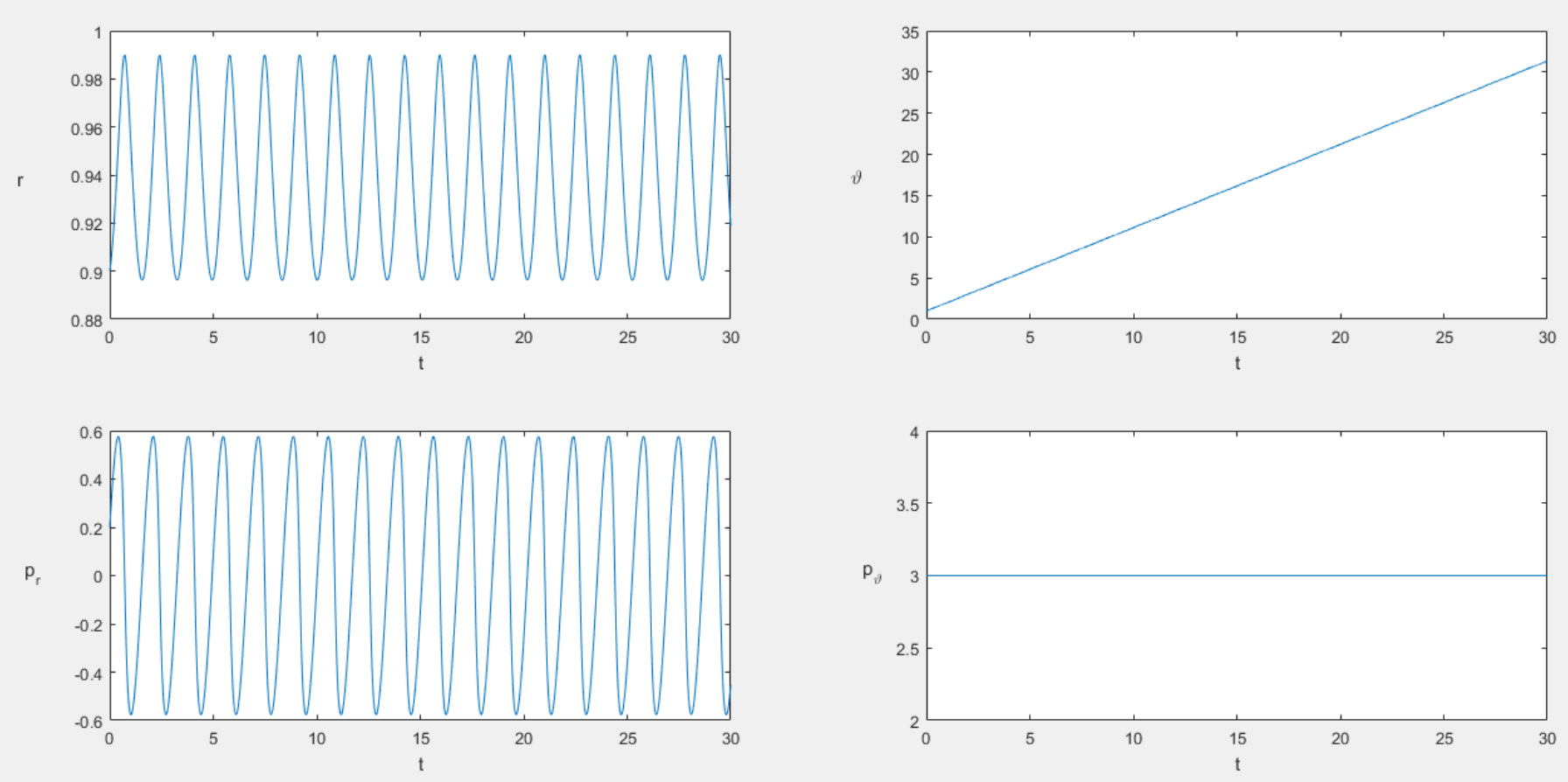

Figure 4.3.9: $m=1, \quad \mathcal{H}_{2, R}(0.9,1,0.2,3)=3.0499544$

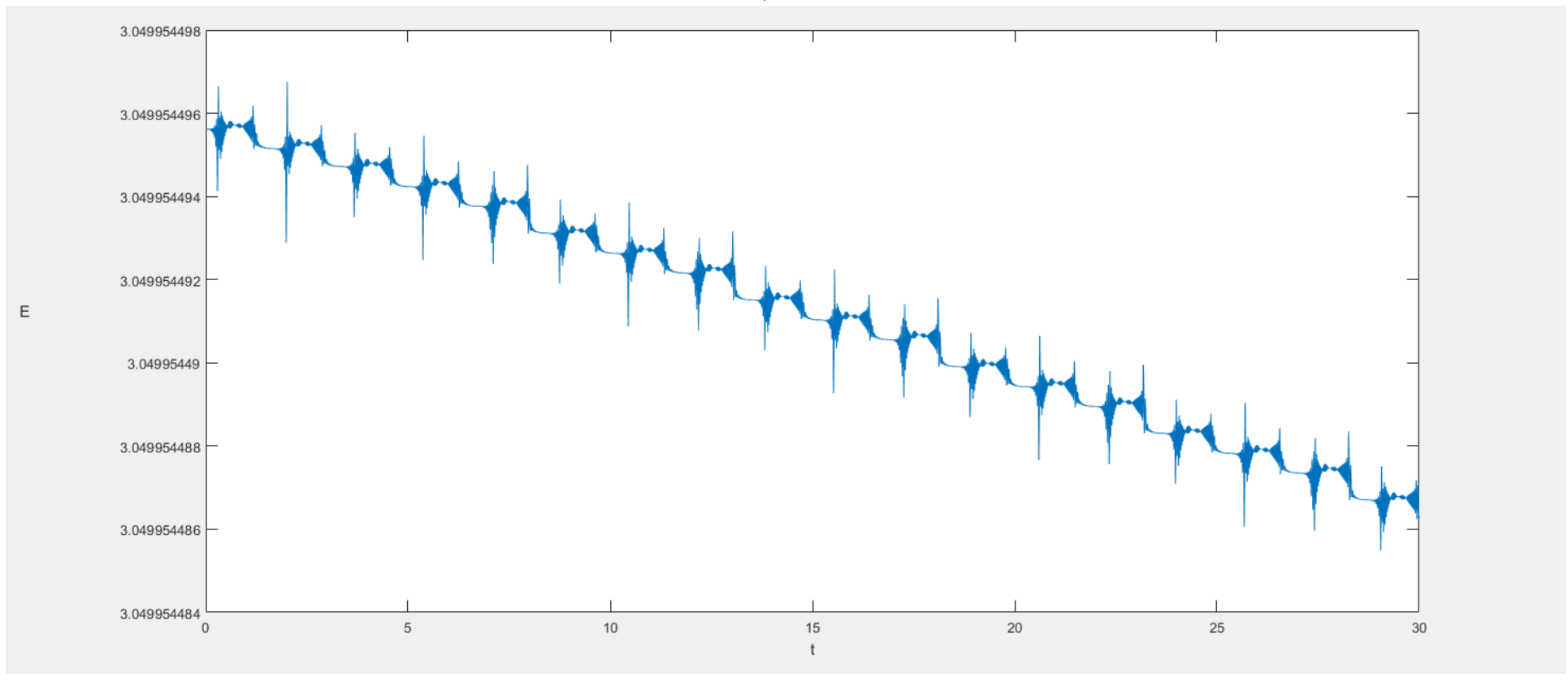

Figure 4.3.10 

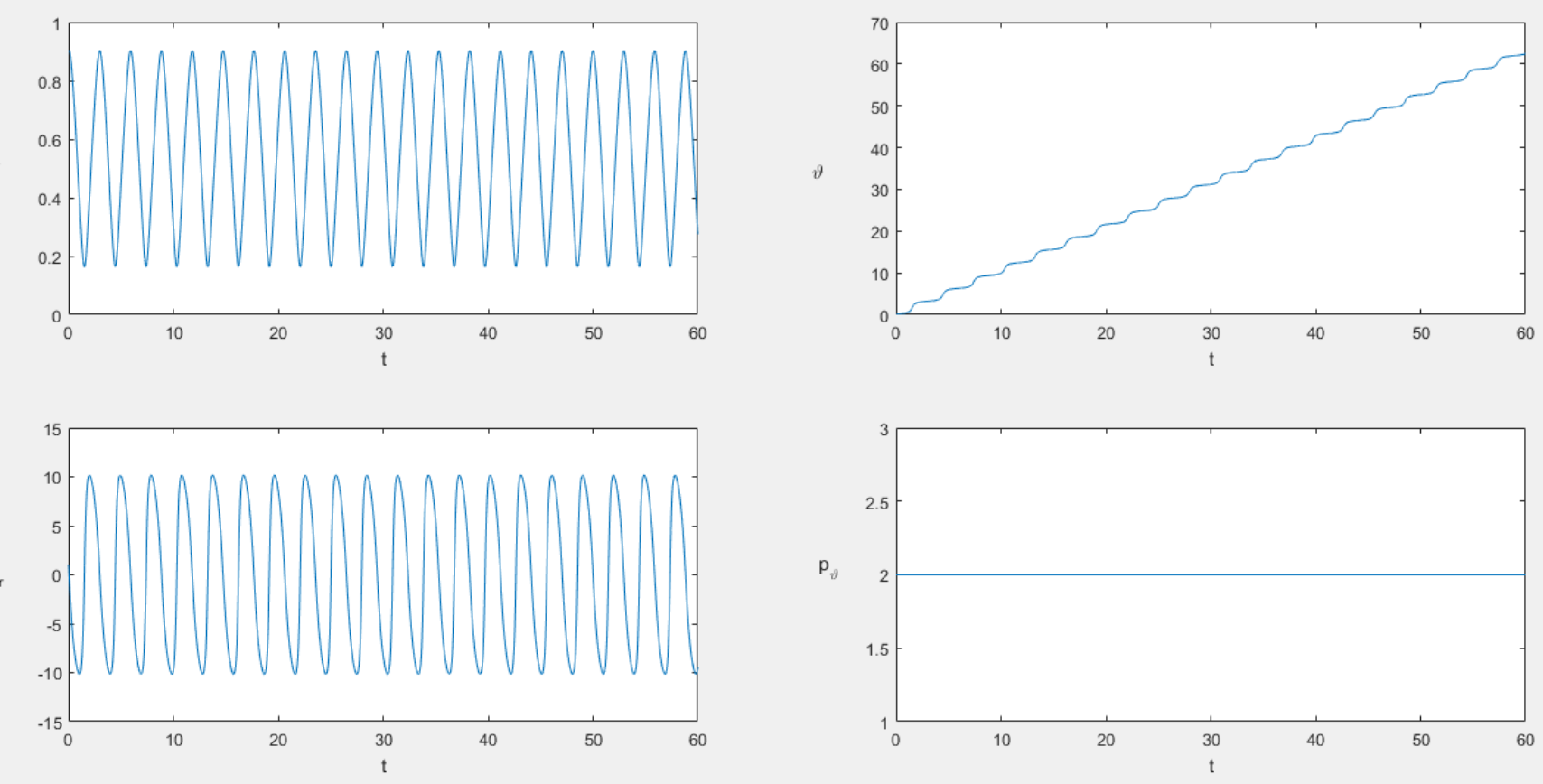

Figure 4.3.11: $m=10, \quad \mathcal{H}_{2, R}(0.9,0.1,1,2)=5.93373$

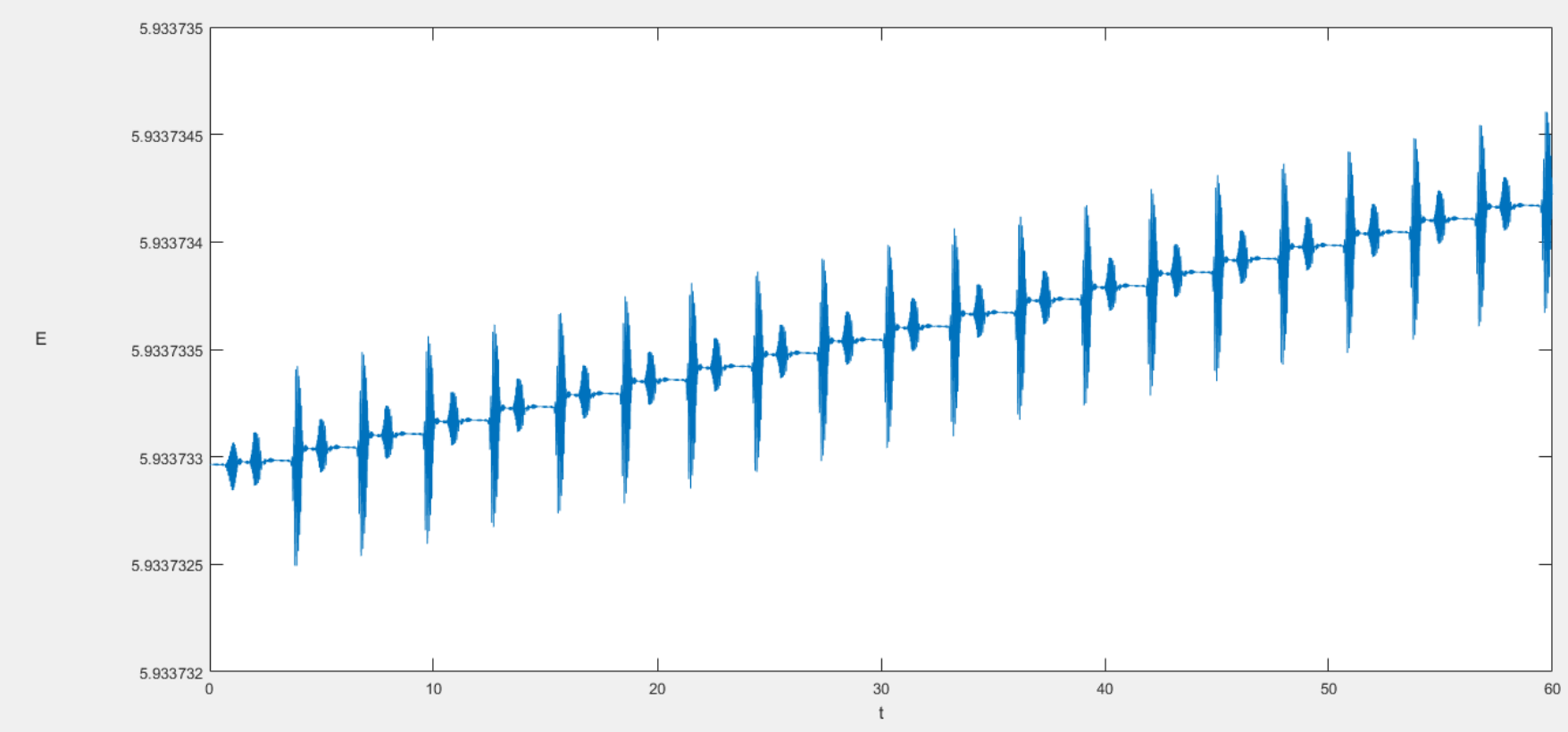

Figure 4.3 .12 
At first glance it is clear that the figures here bare a resemblence to those found with the Harmonic Oscillator. Periodicity is once again strongly suggested. We also notice that some coordinates here imitate quite closely what other coordinates have done in previous examples. Hence we will not repeat any analysis which has been done before. This will be the case with the coordinates $r, p_{r}$, $p_{\vartheta}$ and the plot of E. Having said that additional properties with $r$ and $p_{r}$ is that they both seem to increase as $E$ tends to $\infty$. However one clear difference to the case of the Harmonic Oscillator is that the frequency at which the ocillations occur is much higher here.

Thinking about the path in the two-dimensional plane given by the above figures, it would be very similar to that seen with the two-dimensional Harmonic Oscillator. For larger fluctuations of $\vartheta$ we have a flower thin petals, where a more consistent plot of $\vartheta$ gives a flower with larger petals.

Example 4.3.3. We want to study the dynamics associated with $\mathcal{H} \in C^{1}\left(\mathbb{R}^{6}\right)$ such that

$$
\mathcal{H}(q, p)=\sqrt{m^{2}+\|p\|^{2}}-m+V(q) .
$$

Hamilton's equations follow as

$$
\left\{\begin{array}{l}
\dot{q}_{j}=\frac{p_{j}}{\sqrt{m^{2}+\|p\|^{2}}}, \\
\dot{p}_{j}=-V_{q_{j}}(q)
\end{array} \quad j=1,2,3 .\right.
$$

As before it is useful to have more conserved quantities hence we transform to spherical polar coordinates. Hence we may equivalently consider the function $\mathcal{H}_{3, R} \in$ $C^{1}\left((0, \infty) \times[0, \pi] \times[0,2 \pi) \times \mathbb{R} \backslash\{0\} \times \mathbb{R}^{2}\right)$ such that

$$
\mathcal{H}_{3, R}\left(r, \phi, \theta, p_{r}, p_{\phi}, p_{\theta}\right)=\sqrt{m^{2}+p_{r}^{2}+\frac{p_{\phi}^{2}}{r^{2}}+\frac{p_{\theta}^{2}}{r^{2} \sin ^{2} \phi}}-m+V(r) .
$$

Hamilton's equations are as follows:

$$
\begin{gathered}
\dot{r}=\frac{p_{r}}{\sqrt{m^{2}+p_{r}^{2}+\frac{p_{\phi}^{2}}{r^{2}}+\frac{p_{\theta}^{2}}{r^{2} \sin ^{2} \phi}}}, \\
\dot{\phi}=\frac{p_{\phi}}{r^{2} \sqrt{m^{2}+p_{r}^{2}+\frac{p_{\phi}^{2}}{r^{2}}+\frac{p_{\theta}^{2}}{r^{2} \sin ^{2} \phi}}}, \\
\dot{\theta}=\frac{p_{\theta}}{r^{2} \sin ^{2} \phi \sqrt{m^{2}+p_{r}^{2}+\frac{p_{\phi}^{2}}{r^{2}}+\frac{p_{\theta}^{2}}{r^{2} \sin ^{2} \phi}}}, \\
\dot{p}_{r}=\frac{1}{r^{3}}\left(p_{\phi}^{2}+\frac{p_{\theta}^{2}}{\sin ^{2} \phi}\right) \frac{1}{\sqrt{m^{2}+p_{r}^{2}+\frac{p_{\phi}^{2}}{r^{2}}+\frac{p_{\theta}^{2}}{r^{2} \sin ^{2} \phi}}}-V_{r}(r),
\end{gathered}
$$




$$
\dot{p}_{\phi}=\frac{p_{\theta}^{2} \cos \phi}{r^{2} \sin ^{3} \phi \sqrt{m^{2}+p_{r}^{2}+\frac{p_{\phi}^{2}}{r^{2}}+\frac{p_{\theta}^{2}}{r^{2} \sin ^{2} \phi}}}
$$

and

$$
\dot{p}_{\theta}=0,
$$

implying that $p_{\theta}(t)=\beta_{3}$ for all $t, \beta_{3}$ being some constant. With $\theta$ being a cyclic coordinate of (4.3.38) the claim of Theorem 3.3 .5 is satisfied.

The Hamilton function (4.3.38), $\mathcal{H}_{3, R}$ may be considered as an equivalent function of only four variables $\tilde{\mathcal{H}}_{3, R} \in C^{1}((0, \infty) \times[0,2 \pi) \times \mathbb{R} \backslash\{0\} \times \mathbb{R})$ such that

$$
\tilde{\mathcal{H}}_{3, R}\left(r, \vartheta, p_{r}, p_{\vartheta}\right)=\sqrt{m^{2}+p_{r}^{2}+\frac{\beta_{2}^{2}}{r^{2}}}-m+V(r)
$$

where

$$
\beta_{2}^{2}=p_{\phi}^{2}+\frac{\beta_{3}^{2}}{\sin ^{2} \phi} .
$$

The above function (4.3.43) coincides with the Hamilton function (4.3.22), the twodimensional case of this example. In this comparison we see that $\beta_{2}$ must be the conserved coordinate $p_{\vartheta}$ found in Example 4.3.2.

As with Examples 4.1.3 and 4.2.3, to solve this problem we need the theory of Hamilton-Jacobi. We refer to Example 6.2.3 for the full details and list the main steps here.

Using the theory of Hamilton-Jacobi one can find $\beta_{2}$ to be constant such that

$$
p_{\phi}^{2}+\frac{\beta_{3}^{2}}{\sin ^{2} \phi}=r^{2}\left((E+m-V(r))^{2}-m^{2}-p_{r}^{2}\right)=\beta_{2}^{2}
$$

is true. Through this the solutions are found to be

$$
\begin{gathered}
p_{r}= \pm \sqrt{(E+m-V(r))^{2}-m^{2}-\frac{\beta_{2}^{2}}{r^{2}}} \\
p_{\phi}= \pm \sqrt{\beta_{2}^{2}-\frac{\beta_{3}^{2}}{\sin ^{2} \phi}} \\
\gamma_{1}+t= \pm \int \frac{r(E+m-V(r))}{\sqrt{r^{2}\left((E+m-V(r))^{2}-m^{2}\right)-\beta_{2}^{2}}} \mathrm{~d} r \\
\gamma_{2} \pm \int \frac{\beta_{2}}{r \sqrt{r^{2}\left((E+m-V(r))^{2}-m^{2}\right)-\beta_{2}^{2}}} \mathrm{~d} r= \pm \int \frac{\beta_{2} \sin \phi}{\sqrt{\beta_{2}^{2} \sin ^{2} \phi-\beta_{3}^{2}}} \mathrm{~d} \phi
\end{gathered}
$$


and

$$
\gamma_{3} \pm \int \frac{\beta_{3}}{\sin \phi \sqrt{\beta_{2}^{2} \sin ^{2} \phi-\beta_{3}^{2}}} \mathrm{~d} \phi=\theta
$$

where $\gamma_{i}, i=1,2,3$, is a constant of integration. Whenever the integration is possible $r$ may be found explicitly in terms of through (4.3.47) as with previous examples. The constant $\gamma_{1}$ may then be eliminated with initial conditions.

The remaining coordinates $\phi$ and $\theta$ may be found as before by using the equations (4.3.48) and (4.3.49). Substituting $r$ and then eliminating additional constants obtains $\phi$ by (4.3.48). Repeating the process using both $r$ and $\phi$ with (4.3.49) solves $\theta$.

As with the two-dimensional case, there are some conditions we must check so that the results are well-defined:

(i) $E+m-V(r) \neq 0$,

(ii) $r^{2}\left((E+m-V(r))^{2}-m^{2}\right)-\beta_{2}^{2}>0$,

(iii) $\beta_{2}^{2}-\frac{\beta_{3}^{2}}{\sin ^{2} \phi}>0$.

As with Example 4.2.3, we can follow a similar method to what was seen at the Example 4.1 .3 to deduce that $p_{r} \neq 0$ is sufficient for the above to hold. 


\section{Chapter 5}

\section{Hamilton-Jacobi Theory}

A further approach to studying the dynamics of mechanical systems is through the Hamilton-Jacobi Equation. It is induced from wanting to make Hamilton's equations simpler to solve through a transformation of the coordinates. Here we develop an analogous equation but in the case of the Hamilton function:

$$
\mathcal{H}(q, p)=\psi(p)+V(q),
$$

$\psi \in C N\left(\mathbb{R}^{n}\right) \cap C^{1}\left(\mathbb{R}^{n}\right)$ and $V \in C^{1}\left(\mathbb{R}^{n}\right)$, with Hamilton's equations

$$
\dot{q}_{j}=\frac{\partial \mathcal{H}}{\partial p_{j}}, \quad \dot{p}_{j}=-\frac{\partial \mathcal{H}}{\partial q_{j}}, \quad j=1, \ldots, n,
$$

and the Lagrange function

$$
\mathcal{L}(q, \dot{q})=\psi^{*}(\dot{q})-V(q), \quad \psi^{*} \in C^{2}\left(\mathbb{R}^{n}\right)
$$

and corresponding Lagrange's equations

$$
\frac{\mathrm{d}}{\mathrm{d} t}\left(\frac{\partial \mathcal{L}}{\partial \dot{q}_{j}}\right)=\frac{\partial \mathcal{L}}{\partial q_{j}}, \quad j=1, \ldots, n .
$$

We begin with studying coordinate transformations who have corresponding generating functions. These functions then play a significant role as we then establish our Hamilton-Jacobi equation. Through this we show how the above equations can be equivalently solved.

The main sources we will use are [51] and [26] where a much more extensive presentation of the theory is found for classical Hamiltonians and Lagrangians. We notice that the differentiability of the Hamilton and Lagrange functions is enough for the theory to be carried out as if the functions were classical Hamiltonians and Lagrangians. In particular we also know from Section 3.4 that we can study the associated action function. Hence without proof we only collect the results paramount to our purpose, but with respect to (5.1) and (5.3). 


\subsection{Generating Functions and Canonical Trans- formations}

In classical mechanics using different coordinates may help simplify the problem at hand. They may for example give more cyclic coordinates for the Hamiltonians and Lagrangians introducing conserved quantities to exploit. We took a similar approach in Example 4.1.2 where changing to polar coordinates helped the solving of the relevant Hamilton's and Lagrange's equations. A study in mechanics involves finding these "better" set of coordinates. It uses what are called canonical transformations. Here we present how this theory applies to our dynamics following mainly [26, pages 368 to 375$]$.

We want to transform simultaneously the coordinates $q_{j}$ and $p_{j}, j=1, \ldots, n$, to a new set of independent coordinates $Q_{j}$ and $P_{j}$. This will be determined by invertible transformation equations

$$
Q_{j}=Q_{j}(q, p), \quad P_{j}=P_{j}(q, p)
$$

which relate the two sets of coordinates. To give the new coordinates some context with respect to classical mechanics, the coordinates $Q_{j}$ would correspond to a new set of generalised coordinates where $P_{j}$ represent their associated conjugate momenta. We say that (5.1.1) defines a point transformation of phase space. We will use $Q$ and $P$ to denote the Cartesian product of the $n$ coordinates $Q_{j}$ and $P_{j}$ respectively. As with the coordinates $q$ and $p$, for $I \subset \mathbb{R}$ each variable $Q$ and $P$ will be viewed as mappings in $C^{2}\left(I, \mathbb{R}^{n}\right)$.

For any new coordinate system we transfrom to, we want to ensure that we can still carry out the dynamics already established. Hence we define:

Definition 5.1.1. Suppose a set of $2 n$ coordinates $Q_{j}, P_{j}, j=1, \ldots, n$, satisfy a system of ODE's of the form of (5.2), i.e.

$$
\dot{Q}_{j}=\frac{\partial \mathcal{K}}{\partial P_{j}}, \quad \dot{P}_{j}=-\frac{\partial \mathcal{K}}{\partial Q_{j}}, \quad j=1, \ldots, n,
$$

for some function $\mathcal{K}(Q, P) \in C^{1}\left(\mathbb{R}^{2 n}\right)$. Then $Q_{j}$ and $P_{j}$ are called canonical coordinates.

We can view $\mathcal{K}$ as a new Hamilton function with its own set of Hamilton's equations at (5.1.2). We refer to $\mathcal{K}$ as the transformed Hamilton function or $\mathcal{K}$ Hamilton function defined on the transformed coordinates $Q_{j}$ and $P_{j}$. We also call equations (5.1.2) the transformed Hamilton's equations or $\mathcal{K}$-Hamilton's equations.

The transformed Hamilton's equations at (5.1.2) have the exact same form as the original Hamilton's equations at (5.2). Further the transformed coordinates and 
Hamilton function at (5.1.2) require the same differentiability as their corresponding counterparts $q_{j}, p_{j}$ and $\mathcal{H}$. Therefore the dynamics developed in Chapter 3 can be transitioned freely to work with (5.1.2). We need only replace each $q_{j}$ and $p_{j}$ by $Q_{j}$ and $P_{j}$ respectively, and the function $\mathcal{H}$ by $\mathcal{K}$. Hence to preserve the dynamics of the Hamilton function we would ideally always want to transform to canonical coordinates.

In classical mechanics the transformation equations (5.1.1) allow an explicit dependence on $t$. This is because it considers more general non-autonomous Hamiltonians and Lagrangians. In fact the theory allows the transformed Hamiltonians to also be explicitly dependent on $t$. However studying the dynamics of the Hamilton and Lagrange functions as seen in Chapter 3 relied on them being autonomous. Therefore we insist on the transformation equations on not inducing any non-autonomous transformed Hamilton functions.

We now ask given the transformation of coordinates $(q, p) \mapsto(Q, P)$, when is solving the transformed Hamilton's equations equivalent to solving the original system of Hamilton's equations? This is important as although the new set of coordinates may simplify the problem, we need to ensure that we can look at the solutions for either case as equivalent alternatives to each other. To answer this question we turn to the calculus of variations which was discussed at Section 3.4.

A key result here was that extremals of functionals

$$
G(q, p)=\int_{I} \mathcal{G}(q(t), \dot{q}(t), p(t), \dot{p}(t)) \mathrm{d} t, \quad t \in I \subset \mathbb{R},
$$

may be determined by solving the associated Euler-Lagrange equations. Here $q, p \in$ $C^{2}\left(\bar{I}, \mathbb{R}^{n}\right)$ and for $(q, p)$ and $(\dot{q}, \dot{p})$ fixed, it holds that $\mathcal{G}(., \dot{q}, ., \dot{p}) \in C^{1}(\mathcal{M})$ and $\mathcal{G}(q, ., p,.) \in C^{2}(\mathcal{N})$, where $\mathcal{M} \times \mathcal{N}$ form an open neighbourhood of

$$
\{(t, q(t), p(t), \dot{q}(t), \dot{p}(t)) \mid t \in \bar{I}\} \subset \mathbb{R}^{4 n+1},
$$

the 1-graph of $(q, p)$. We refer back to Section 3.4 for the full details. However, when $\mathcal{G}$ is defined as

$$
\begin{aligned}
\mathcal{G}(q(t), \dot{q}(t), p(t), \dot{p}(t)) & :=p(t) \cdot \dot{q}(t)-\mathcal{H}(q(t), p(t)) \\
& =p(t) \cdot \dot{q}(t)-\psi(p(t))-V(q(t))),
\end{aligned}
$$

then solving the Euler-Lagrange equations is equivalent to solving the associated Hamilton's equations. It is through this connection one can prove the following:

Proposition 5.1.2. (see [26, Chapter 9.1])

Let $(q, p)$ and $(Q, P)$ be two sets of canonical coordinates with the associated Hamilton and transformed Hamilton functions $\mathcal{H}(q, p)$ and $\mathcal{K}(Q, P)$ respectively. Then 
solving Hamilton's equations is equivalent to solving the transformed Hamilton's equations whenever

$$
p(t) \cdot \dot{q}(t)-\mathcal{H}(q(t), p(t))=P(t) \cdot \dot{Q}(t)-\mathcal{K}(Q(t), P(t))+\frac{\mathrm{d} \mathcal{F}}{\mathrm{d} t} .
$$

At equation (5.1.3) it is assumed that $q, p, Q, P \in C^{1}\left(I, \mathbb{R}^{n}\right)$ and $\mathcal{F}(q, p, Q, P, t) \in$ $C^{2}\left(\mathbb{R}^{4 n+1}\right)$.

With this we define:

Definition 5.1.3. Let $(q, p)$ and $(Q, P)$ be two sets of canonical coordinates with their associated Hamilton functions $\mathcal{H}(q, p)$ and $\mathcal{K}(Q, P)$ respectively. We call a transformation $(q, p) \mapsto(Q, P)$ a canonical transformation if the solutions of the Hamilton and $\mathcal{K}$-Hamilton equations are equivalent.

Through introducing and inverting the original transformation equations (5.1.1), equation (5.1.3) may be used to express $\mathcal{F}$ in terms of one set of coordinates from $(q, p)$, and a further set of coordinates from $(Q, P)$. For example we may have $\mathcal{F}=\mathcal{F}(p, Q, t)$. In fact a useful property is gained by this: suppose we did not know the transformation equations for a given canonical transformation. But, we do have the associated function $\mathcal{F}$ given by (5.1.3) such that it is partially dependent on the old and new set of coordinates. There is then a way to determine the transformation equations. We show how this can be done shortly. But first, because of this property of $\mathcal{F}$ in generating the transformation equations, we define:

Definition 5.1.4. We call the function $\mathcal{F}$ satisfiying equation (5.1.3) the generating function.

Below is an example to demonstrate how the transformation equations may be derived from a given generating function:

Example 5.1.5. Suppose the generating function $\mathcal{F}$ is a function of $q$ and $P$ out of the four possible sets of canonical coordinates. Further let it have the form

$$
\mathcal{F}(q, Q, P, t)=\tilde{\mathcal{F}}(q, P, t)-Q \cdot P,
$$

where $\tilde{\mathcal{F}}$ is a further function of $q, P$ and $t$. Substituting equation (5.1.4) into equation (5.1.3) gives

$$
\begin{aligned}
p \cdot \dot{q}-\mathcal{H}(q, p) & =P \cdot \dot{Q}-\mathcal{K}(Q, P)+\frac{\mathrm{d}}{\mathrm{d} t}(\tilde{\mathcal{F}}(q, P, t)-Q \cdot P) \\
& =-\mathcal{K}(Q, P)+\sum_{j=1}^{n} \frac{\partial \tilde{\mathcal{F}}}{\partial q_{j}} \dot{q}_{j}+\sum_{j=1}^{n} \frac{\partial \tilde{\mathcal{F}}}{\partial P_{j}} \dot{P}_{j}+\frac{\partial \tilde{\mathcal{F}}}{\partial t}-Q \cdot \dot{P}
\end{aligned}
$$


where we have used the product and chain rules of differentiation. Rearranging and recalling that $q_{j}$ and $P_{j}$ vary independently as coordinates gives

$$
p_{j}=\frac{\partial \tilde{\mathcal{F}}}{\partial q_{j}}(q, P, t) \quad \text { and } \quad Q_{j}=\frac{\partial \tilde{\mathcal{F}}}{\partial P_{j}}(q, P, t), \quad j=1, \ldots, n
$$

and

$$
\mathcal{K}(Q, P)=\mathcal{H}(q, p)+\frac{\partial \mathcal{F}}{\partial t} .
$$

Equation (5.1.5) now allows us to obtain the transformation equations for the canonical transformation that (5.1.4) corresponds to.

By the first equation of (5.1.5) we have $n$ relations giving the coordinate $p_{j}$ as functions of $q_{l}, P_{l}, l=1, \ldots, n$, and $t$. Through this we can find each coordinate $P_{j}$ in terms of $q_{l}$ and $p_{j}$ by inverting the equation (assuming that this is possible). This gives the first transformation equation. Substituting our new $P_{j}$ into the second equation of (5.1.5) gives the coordinates $Q_{j}$ as functions of $q_{l}$ and $p_{l}$. This is the second transformation equation.

Having found the transformation equations, the transformed Hamilton function $\mathcal{K}$ may then be determined by equation (5.1.6). This is done using the transformation equations to express the known Hamilton and generating functions on the right-hand side of (5.1.6) as functions of $Q_{j}$ and $P_{j}$.

We learn from Example 5.1.5 that derivatives of the generating function allow the deduction of the transformation equations. For convenience we refer to these derivatives as the generating function derivatives. Additionally we gained an equation of the form of (5.1.6) which relates the Hamilton function to the transformed Hamilton function. Through this we were then able to find the new $\mathcal{K}$-Hamilton function.

Other possibilities for transforming coordinates using generating functions other than (5.1.4) also exist. For example, one can obtain generating function derivatives so that each $q_{j}$ is in terms of $p_{j}$ and $Q_{j}$, instead of $p_{j}$ in terms of $q_{j}$ and $P_{j}$ as it was at (5.1.5). To obtain four such transformations, one can collect the permutations of considering the different ways $\mathcal{F}$ may be expressed as a function of two of the four sets of possible coordinates $q, p, Q$ and $P$. This forms four basic canonical transformations. We present their properties below, similarly seen at $[26$, page 373]: 
Table 5.1.1: Four Basic Canonical Transformations

\begin{tabular}{|c|c|c|}
\hline Generating Functions & \multicolumn{2}{|c|}{ Generating Function Derivatives } \\
\hline $\mathcal{F}=\mathcal{F}_{1}(q, Q, t)$ & $p_{j}=\frac{\partial \mathcal{F}_{1}}{\partial q_{j}}$ & $P_{j}=-\frac{\partial \mathcal{F}_{1}}{\partial Q_{j}}$ \\
\hline $\mathcal{F}=\mathcal{F}_{2}(q, P, t)-Q \cdot P$ & $p_{j}=\frac{\partial \mathcal{F}_{2}}{\partial q_{j}}$ & $Q_{j}=\frac{\partial \mathcal{F}_{2}}{\partial P_{j}}$ \\
\hline $\mathcal{F}=\mathcal{F}_{3}(p, Q, t)+q \cdot p$ & $q_{j}=-\frac{\partial \mathcal{F}_{3}}{\partial p_{j}}$ & $P_{j}=-\frac{\partial \mathcal{F}_{3}}{\partial Q_{j}}$ \\
\hline $\mathcal{F}=\mathcal{F}_{4}(p, P, t)+q \cdot p-Q \cdot P$ & $q_{j}=-\frac{\partial \mathcal{F}_{2}}{\partial p_{j}}$ & $Q_{j}=\frac{\partial \mathcal{F}_{4}}{\partial P_{j}}$ \\
\hline
\end{tabular}

Note it was actually the basic canonical transformation from the second row of Table 5.1.1 that we covered in Example 5.1.5 where we denoted $\mathcal{F}_{2}$ as $\tilde{\mathcal{F}}$.

It will be useful later to know that deriving the generating function derivatives as at Example 5.1.5 for each function $\mathcal{F}$ in Table 5.1.1 gives the equation

$$
\mathcal{K}(Q, P)=\mathcal{H}(q, p)+\frac{\partial \mathcal{F}}{\partial t}
$$

each time. This equation appeared in the form of (5.1.6) at Example 5.1.5.

A concern with canonical transformations is ensuring that the transformation equations (5.1.1) induce autonomous transformed Hamilton functions. Returning to Example 5.1.5 this problem may arise from the inverting of equations at (5.1.5) where $t$ as an explicit term may play a role in defining the transformed coordinates. It is at this point we could obtain non-autonomous transformed Hamilton functions for which our dynamics from Chapter 3 would not apply to.

The problem may originate from allowing the generating function to be explicitly dependent on $t$. However the following example shows that autonomous transformation equations can be obtained from generating functions of the form $\mathcal{F}(., ., t)$.

Example 5.1.6. Let $(q, p) \in \mathbb{R}^{2 n}$ be a set of canonical coordinates with the associated Hamilton function $\mathcal{H}(q, p) \in C^{1}\left(\mathbb{R}^{2 n}\right)$ given as

$$
\mathcal{H}(q, p)=\frac{1}{\alpha}\|p\|^{\alpha}+\frac{1}{\alpha^{*}}\|q\|^{\alpha^{*}}, \quad 1<\alpha \leq 2, \quad \alpha^{*}=\frac{\alpha}{\alpha-1} .
$$


Suppose we want to make a canonical transformation to a set of canonical coordinates $(Q, P) \in \mathbb{R}^{2 n}$ with the associated generating function being $\mathcal{F}_{1}(q, Q, t)=q \cdot Q+f(t)$. Here it is assumed that $f$ is some linear function on $t$ defined as $f(t):=c t+a, a, c \in$ $\mathbb{R}$. Looking back to the first row of Table 5.1.1 the generating function derivatives of $\mathcal{F}_{1}$ follow as

$$
p_{j}=\frac{\partial \mathcal{F}_{1}}{\partial q_{j}}=Q_{j} \quad \text { and } \quad P_{j}=-\frac{\partial \mathcal{F}_{1}}{\partial Q_{j}}=-q_{j} .
$$

By equation (5.1.6) we also know that the transformed Hamilton function is given by

$$
\mathcal{K}(Q, P)=\mathcal{H}(q, p)+\frac{\partial \mathcal{F}_{1}}{\partial t}
$$

Rearranging the equations at (5.1.8) the associated transformation equations for the canonical transformation $(q, p) \mapsto(Q, P)$ are found to be

$$
Q_{j}=p_{j} \quad \text { and } \quad P_{j}=-q_{j} .
$$

Hence by (5.1.9) the transformed Hamilton function follows as

$$
\mathcal{K}(Q, P)=\frac{1}{\alpha}\|Q\|^{\alpha}+\frac{1}{\alpha^{*}}\|P\|^{\alpha^{*}}+c
$$

where the constant $c$ is the derivative of $f$.

So Example 5.1.6 shows the possibility of a generating function $\mathcal{F}(., ., t)$ inducing autonomous transformation equations and transformed Hamilton functions. To ensure that we always have this situation, it would be sufficient that $\mathcal{F}$ has the specific form suggested by the following proposition:

Proposition 5.1.7. Let $(q, p)$ be a set of canonical coordinates with $(Q, P)$ as the transformed coordinates obtained through a canonical transformation. Further let

$$
\mathcal{F}=\tilde{\mathcal{F}}(q, p, Q, P)+f(t), \quad \mathcal{F} \in C^{2}\left(\mathbb{R}^{4 n+1}\right),
$$

be a generating function where $\tilde{\mathcal{F}} \in C^{2}\left(\mathbb{R}^{4 n}\right)$ and $f$ is a linear function explicitly dependent on $t$. Then the transformed Hamilton function as well as the transformation equations induced by the canonical transformation are autonomous.

Proof. Looking to the generating function derivatives in Table 5.1.1, it is clear that (5.1.11) will always induce transformation equations independent of $t$. This is because $f(t)$ will always be viewed as constant when taking partial derivatives of $\mathcal{F}$ with respect to any of the coordinates, leaving the partial derivative of $\tilde{\mathcal{F}}(q, p, Q, P)$.

Being a linear function we know that $\frac{\partial \mathcal{F}}{\partial t}=f^{\prime}(t)$ would always be the constant c. Through this equation (5.1.7) appears as

$$
\mathcal{K}(Q, P)=\mathcal{H}(q, p)+c .
$$


Hence knowing already that the transformation equations are autonomous, the transformed Hamilton function is also guaranteed to be autonomous.

When considering a canonical transformation $(q, p) \mapsto(Q, P)$ from this point onwards, we assume that it does always induce autonomous transformation equations as well as Hamilton functions.

Before concluding this section its good to know that coordinate transformations can also be used to simplify problems when dealing with the Lagrange function. We have already seen examples of this in Chapter 4 where we transformed to polar and spherical polar coordinates. Following [26, Chapter 9.1 and 9.2] we can write such a transformation as a mapping $q \mapsto Q$ where the new coordinates $Q_{j}$ are determined by the old coordinates $q_{j}$ by invertible transformation equations of the form

$$
Q_{j}=Q_{j}\left(q_{1}, \ldots, q_{n}\right), \quad j=1, \ldots, n
$$

Such transformations we call point transformations.

To relate this to previous discussions one can find that canonical transformations act as a generalisation of point transformations: for the canonical transformation $(q, p) \mapsto(Q, P)$ consider the generating function from the second row of Table 5.1.1 with

$$
\mathcal{F}_{2}(q, P):=\sum_{j=1}^{n} f_{j}\left(q_{1}, \ldots, q_{n}\right) P_{j} .
$$

At (5.1.14) the functions $f_{j}$ are independent and invertible defined on the configuration space. By the associated generating function derivatives to (5.1.14) the coordinates $Q_{j}$ follow as

$$
Q_{j}=\frac{\partial \mathcal{F}_{2}}{\partial P_{j}}=f_{j}\left(q_{1}, \ldots, q_{n}\right)
$$

which is a point transformation when compared to (5.1.13). The functions $f_{j}$ were chosen arbitrarily hence all point transformations must be canonical. 


\subsection{The Hamilton-Jacobi Equation}

When discussing canonical transformations in the previous section we showed how one can make a change of coordinates $(q, p) \mapsto(Q, P)$ so that solving Hamilton's equations in this new coordinate system is equivalent to doing so in terms of the original coordinates. One can build on this and derive the Hamilton-Jacobi equation. The advantage here is that it requires the transformed Hamilton function to be zero. Hence solving the transformed Hamilton's equations is trivial giving the transformed coordinates as constant. Through this one can find that solving the Hamilton-Jacobi equation is equivalent to solving Hamilton's equations and so provides an additional, and some times easier, approach to studying the associated dynamics.

We first explain how the Hamilton-Jacobi equation is obtained for a given Hamilton function. Following this we will show how this relates to canonical transformations and the solving of Hamilton's equations.

As before the Hamilton and Lagrange functions satisfy what is required of classical Hamiltonians and Lagrangians for this work to be carried out in classical mechanics. Hence we do not present the full details here but only the main steps and results important to us. For a more in depth explanation we refer to [51, Chapter 7] and [26, Chapter 10].

Recall from Section 3.4 that the calculus of variations allowed us to identify solutions of Lagrange's equations as extremals of the functional

$$
S(q)=\int_{I} \mathcal{L}(q(s), \dot{q}(s)) \mathrm{d} s, \quad I=(a, b) \subset \mathbb{R},
$$

where $q \in C^{2}\left(\bar{I}, \mathbb{R}^{n}\right)$ and $\mathcal{L}$ is the corresponding Lagrange function. To distinguish (5.2.1) from what was done in Section 3.4 notice we have denoted the above functional by $S(q)$. To be clear here $S$ is considered as a function of the extremals, not any point in the configuration space.

An interpretation of extremals of (5.2.1) were that they are paths in the space plotting $q(t)$ against $t$ constrained to vary between the two fixed points $q(a)$ and $q(b)$. Now we choose to consider when extremals $q(t)$ are fixed at one endpoint $q(a)$, and are allowed to vary at the other endpoint. Because of this we now consider $S$ as a function of $t$ also, i.e. $S=S(q, t)$. Following [51] one can deduce $S$ to satisfy

$$
\frac{\partial S}{\partial q_{j}}=p_{j}
$$

Through this it can be found that

$$
\frac{\partial S}{\partial t}=-\mathcal{H}(q, p) .
$$


In fact $S$ is found to have the form

$$
S(q, t)=W(q)-E t
$$

where $E$ is the conserved value of the Hamilton function. The function $W \in C^{2}\left(\mathbb{R}^{n}\right)$ is defined as

$$
W(q):=\int \sum_{l=1}^{n} p_{l} \dot{q}_{l} \mathrm{~d} t .
$$

For future convenience we adopt the naming convention of $W$ and $S$ as seen at [26]:

Definition 5.2.1. The functional $S$ is called Hamilton's principal function and $W$ is called Hamilton's characteristic function.

With (5.2.2) as a new characterisation of the coordinate $p_{j}$ we can use this with (5.2.3) to define

Definition 5.2.2. Suppose $S$ satisfies equation (5.2.2). Then we have

$$
\mathcal{H}\left(q_{1}, \ldots, q_{n} ; \frac{\partial S}{\partial q_{1}}, \ldots, \frac{\partial S}{\partial q_{n}}\right)+\frac{\partial S}{\partial t}=0 .
$$

Using the form of $S$ at (5.2.4) we alternatively have

$$
\mathcal{H}\left(q_{1}, \ldots, q_{n} ; \frac{\partial W}{\partial q_{1}}, \ldots, \frac{\partial W}{\partial q_{n}}\right)=E .
$$

Both are equivalent forms of the Hamilton-Jacobi equation. At (5.2.5) it may be read as a first-order partial differential equation of $S$ with $n+1$ variables. Similarly (5.2.6) may be read as a first-order partial differential equation of $W$ but with $n$ variables.

To summarise we have deduced that the calculus of variations allows us to interpret the conservation of the Hamilton function, $\mathcal{H}(q, p)=E$, in a different way, specifically of the form of the Hamilton-Jacobi equation at (5.2.6). The usefulness of this representation becomes apparent as we now employ the help of [26].

Here it is seen that the Hamilton-Jacobi equation is related to

$$
\mathcal{K}(Q, P)=\mathcal{H}(q, p)+\frac{\partial S}{\partial t},
$$

an equation we obtained in the previous section when making a basic canonical transformation $(q, p) \mapsto(Q, P)$ with a generating function $S$. Comparing (5.2.5) to (5.2.7), their equivalence would deduce the transformed Hamilton function $\mathcal{K}$ to be zero. Hence the suggested connection between (5.2.5) and (5.2.7) is that the 
Hamilton-Jacobi equation in fact corresponds to a basic canonical transformation giving a $\mathcal{K}$-Hamilton function with value zero. As mentioned before this would then be very useful as the $\mathcal{K}$-Hamilton's equations would then be trivial to solve, i.e. solving

$$
\dot{Q}_{j}=\frac{\partial \mathcal{K}}{\partial P_{j}}=0, \quad \dot{P}_{j}=-\frac{\partial \mathcal{K}}{\partial Q_{j}}=0, \quad j=1, \ldots, n,
$$

gives the coordinates $Q_{j}$ and $P_{j}$ as constants. Recall the main drive behind canonical transformations was to obtain an equivalent system to Hamilton's equations that is easier to solve. This aim is most certainly achieved with the system at (5.2.8) above.

Following what we learnt in Section 5.1 we now ask what would be the corresponding generating function? We already have a clue in our comparison of (5.2.5) and (5.2.7) where we can spot that $S$ as at (5.2.4) would have to be the generating function. Equation (5.2.2) would then be one of the corresponding generating function derivatives. Looking back to Table 5.1.1, we see that $S$ and its derivative (5.2.2) compare well to its second row with $\mathcal{F}_{2}$ and its derivatives as a part of the second basic canonical transformation.

Remark 5.2.3. A more observant look at Table 5.1.1 also suggests that $S$ may also be chosen to be $\mathcal{F}_{1}$. This is entirely true and the remaining results may be carried out analogously. However, the main point here is to have the existence of some generating function so that $\mathcal{K}=0$. The generating function need not be unique, as long as it serves our purpose. The source [26] selects $\mathcal{F}_{2}$ and so we follow suit here but it is good to remember that another choice is possible.

A concern does arise from this however: in general $\mathcal{F}_{2}$ is a function of $q, P$ and $t$ where the functional $S$ we know to only depend on $q$ and $t$. This problem is resolved by recollecting that the Hamilton-Jacobi equation (5.2.6) is a first-order partial differential equation of $n$ variables. From this it is deduced that $W$ as a solution of (5.2.6) is of the form

$$
W=W\left(q_{1}, \ldots, q_{n} ; E, \beta_{2}, \ldots, \beta_{n}\right)
$$

where $\beta_{j}, j=2, \ldots, n$ are constants of integration. This gives $S$ as

$$
S=S\left(q_{1}, \ldots, q_{n} ; E, \beta_{2}, \ldots, \beta_{n} ; t\right)=W\left(q_{1}, \ldots, q_{n} ; E, \beta_{2}, \ldots, \beta_{n}\right)-E t .
$$

Identifying the constants $E, \beta_{2}, \ldots, \beta_{n}$ as the coordinates $P_{j}$, i.e.

$$
P_{j}= \begin{cases}E, & \text { if } j=1, \\ \beta_{j}, & \text { if } j=2, \ldots, n,\end{cases}
$$


we maintain the necessary structure of $S(q, P, t)$. For convenience we may at times write $E$ as $\beta_{1}$.

To summarise: we read $S$ as a generating function for the second basic canonical transformation from the second row of Table 5.1.1. Its generating function derivatives follow as

$$
p_{j}=\frac{\partial S}{\partial q_{j}}(q ; E, \beta ; t) \quad \text { and } \quad Q_{j}=\frac{\partial S}{\partial P_{j}}(q ; E, \beta ; t), \quad \beta=\left(\beta_{2}, \ldots, \beta_{n}\right) .
$$

We know that $S$ satisfies the Hamilton-Jacobi equation whose structure implies that the corresponding canonical transformation must induce a transformed Hamilton function $\mathcal{K}$ that has a value of zero. The transformed Hamilton's equations can then be trivially solved to deduce the transformed coordinates $Q_{j}$ and $P_{j}$ as constants:

$$
Q_{j}=\gamma_{j} \quad \text { and } \quad P_{1}=E, P_{m}=\beta_{m}, \quad \text { for } \quad m=2, \ldots, n .
$$

It still remains to answer how does this help us solve Hamilton's equations for a given Hamiton function? To do this we employ the initial conditions $q\left(t_{0}\right)=q_{0}$ and $p\left(t_{0}\right)=p_{0}$ and use them with the derivatives at (5.2.11). This allows us to fully determine each coordinate $q_{j}$ and $p_{j}$ in terms of $E$ and the initial values $q_{0}$ and $p_{0}$.

Looking to the first derivative of $S$ at (5.2.11) we have $n$ relations for $p_{j}$ as functions of $q_{l}, l=1, \ldots, n, E$ and $\beta_{m}, m=2, \ldots, n$. Using the initial conditions with (5.2.11) we can determine the constants $\beta_{m}$ in terms of the initial values $t_{0}, q_{0}$, $p_{0}$ as well as $E$.

Now we look to the second generating function derivative at (5.2.11). We have this time $n$ relations giving $Q_{j}$ (or $\gamma_{j}$ ) as functions of $q_{l}, E$ and $\beta_{m}$. However we also now have each $\beta_{m}$ as functions of $E$ and the initial values. Therefore this equation in fact can give the constants $\gamma_{j}$ in terms of the inital values and $E$. This would leave an equation of the form

$$
\gamma_{j}\left(q_{0}, p_{0}, t_{0}, E\right)=\frac{\partial S}{\partial P_{j}}\left(q ; E, \beta\left(q_{0}, p_{0}, t_{0}, E\right) ; t\right)
$$

which may then be inverted (assuming this is possible) to obtain each $q_{j}$ as a function of $E$ and the initial values, i.e. we have

$$
q_{j}=q_{j}\left(E, q_{0}, p_{0}, t_{0}\right) .
$$

This leaves each $q_{j}$ as fully determined. Substituting (5.2.13) into the first derivative of $S$ at (5.2.11) would then fully determine each $p_{j}$, i.e.

$$
p_{j}=p_{j}\left(E, q_{0}, p_{0}, t_{0}\right) \text {. }
$$


What we have shown is that solving the Hamilton-Jacobi equation for $S$ allows us to fully determine the coordinates $q_{j}$ and $p_{j}$ through the manipulation of the generating function derivatives. Implicitly this solves Hamilton's equations. We know this since we have used a canonical transformation $(q, p) \mapsto(Q, P)$ where from Proposition 5.1.2 we know that solving Hamilton's equations in terms of either coordinate system is equivalent. Using the associated generating function derivatives at (5.2.11) with the set initial conditions of the problem, we are able to fully determine each transformed coordinate $Q_{j}=\gamma_{j}$ and $\beta_{j}=P_{j}$. These are the solutions to the transformed Hamilton's equations who have a dependence on the original coordinates $q_{j}$ and $p_{j}$. Remember it is the determination of these coordinates that we actually desire. Hence by manipulating these conserved quantites we were able solve the coordinates $q_{j}$ and $p_{j}$, the solutions to the original Hamilton's equations. In this sense we have an equivalence between solving Hamilton's equations, a system of $2 n$ firstorder ordinary differential equations, and solving the Hamilton-Jacobi equation, a first-order partial differential equation.

There is a possible disadvantage to adopting the Hamilton-Jacobi approach. It may happen that solving it for $S$ or $W$ may not always be easy to do. In classical mechanics there is a way to deal with this is by exploiting separable coordinates. This can be found at [26, Chapters 10.4 and 10.5]. Going with the usual routine we would like to form an analogue to this for our dynamics. We begin this next discussion with our definition for separable coordinates:

Definition 5.2.4. We call a coordinate $q_{j}$ separable if Hamilton's principal function $S$ can be written as

$$
S(q ; E, \beta ; t)=W_{j}\left(q_{j} ; E, \beta\right)+\tilde{W}\left(q_{l} ; E, \beta\right)-E t, \quad W_{j} \in C^{2}(\mathbb{R}), \tilde{W} \in C^{2}\left(\mathbb{R}^{n-1}\right),
$$

such that the associated Hamilton-Jacobi equation can be split into two further equations, one dependent only on $q_{j}$ and $W_{j}$, the other dependent on the remaining $q_{l}$ and $\tilde{W}$. Here $q_{l}$ denotes all coordinates other than $q_{j}$.

For our own convenience we will continue to use the notation $q_{l}$ and analogously $p_{l}$ throughout the rest of this discussion.

To spot a separable coordinate $q_{j}$, it helps when the Hamilton function can be rearranged so that it is of the form

$$
\mathcal{H}(q, p)=\mathcal{H}\left(q_{l}, p_{l}, f_{j}\left(q_{j}, p_{j}\right)\right)=E
$$

where $f_{j}$ is some function of only $q_{j}$ and $p_{j}$. This means that the Hamilton function can be written so that the coordinates $q_{j}$ and $p_{j}$ can be segregated into a function $f_{j}$. 
For example let us recall the Hamilton function $\mathcal{H}_{3}\left(r, \phi, \theta, p_{r}, p_{\phi}, p_{\theta}\right)$ from Section 4.1:

$$
\mathcal{H}_{3}\left(r, \phi, \theta, p_{r}, p_{\phi}, p_{\theta}\right)=\frac{1}{\alpha}\left(p_{r}^{2}+\frac{p_{\phi}^{2}}{r^{2}}+\frac{\beta_{3}^{2}}{r^{2} \sin ^{2} \phi}\right)^{\frac{\alpha}{2}}+\frac{1}{\alpha^{*}} r^{\alpha^{*}}
$$

where $\beta_{3}$ was constant. This function may be rewritten as

$$
\mathcal{H}_{3}\left(r, \phi, \theta, p_{r}, p_{\phi}, p_{\theta}\right)=\frac{1}{\alpha}\left(p_{r}^{2}+\frac{1}{r^{2}}\left(p_{\phi}^{2}+\frac{\beta_{3}^{2}}{\sin ^{2} \phi}\right)\right)^{\frac{\alpha}{2}}+\frac{1}{\alpha^{*}} r^{\alpha^{*}} .
$$

By defining a further function

$$
f\left(\phi, p_{\phi}\right):=p_{\phi}^{2}+\frac{\beta_{3}^{2}}{\sin ^{2} \phi},
$$

the coordinates $\phi$ and $p_{\phi}$ are now segragated within $f$. Hence $\mathcal{H}_{3}$ may now be written as

$$
\mathcal{H}_{3}\left(r, \phi, \theta, p_{r}, p_{\phi}, p_{\theta}\right)=\mathcal{H}_{3}\left(r, \theta, p_{r}, p_{\theta}, f\left(\phi, p_{\phi}\right)\right),
$$

giving the form as seen at (5.2.15).

Returning to the Hamilton function at (5.2.15), we show how having this form can help find a separable coordinate. Suppose $S$ appears as at (5.2.14) and that Hamilton function may be written as at (5.2.15). Then the corresponding HamiltonJacobi equation would appear as:

$$
\mathcal{H}\left(q_{l} ; \frac{\partial \tilde{W}}{\partial q_{l}}, f_{j}\left(q_{j}, \frac{\partial W_{j}}{\partial q_{j}}\right)\right)=E .
$$

Suppose (5.2.16) can then rearranged to obtain an equation of the form

$$
f_{j}\left(q_{j}, \frac{\partial W_{j}}{\partial q_{j}}\right)=g_{j}\left(q_{l}, \frac{\partial \tilde{W}}{\partial q_{l}}, E\right)
$$

where $g$ is some function of $q_{l}, \frac{\partial \tilde{W}}{\partial q_{l}}$ and $E$. We can now take a similar argument to what was used to solve the three-dimensional examples from Chapter 4: the lefthand side of (5.2.17) depends only on $q_{j}$ where the right-hand side is independent of $q_{j}$. Each coordinate $q_{j}$ and $q_{l}$ can be varied independently hence (5.2.17) can only be true if both sides of the equation are equal to the same constant, i.e.

$$
f_{j}\left(q_{j}, \frac{\partial W_{j}}{\partial q_{j}}\right)=\beta_{j},
$$

and

$$
g_{j}\left(q_{l}, \frac{\partial \tilde{W}}{\partial q_{l}}, E\right)=\beta_{j}
$$


We see it is at this point the constants $\beta_{2}, \ldots, \beta_{m}$ may be introduced into the problem. Hence what we have achieved is that having taken $S$ to be as at (5.2.14), the form of the Hamilton function at (5.2.15) has allowed us to split it into two equations, namely equation (5.2.18) who depends only on $q_{j}$ and $W_{j}$, the other being equation (5.2.19) who depends on $q_{l}$ and $\tilde{W}$. This is assuming of course that the rearrangement to give (5.2.17) is possible. Given all of this, the coordinate $q_{j}$ is separable by Definition 5.2.4.

We originally wanted to find separable coordinates so that it may help with the solving of the Hamilton-Jacobi equation. We now know that in an ideal situation where $q_{j}$ is separable, it may be possible that we may equivalently consider a further two equations of the form (5.2.18) and (5.2.19) to solve for $S$. Being dependent on less variables, we would hope that both equations would be easier to solve for $W_{j}$ and $\tilde{W}$ rather than solving the Hamilton-Jacobi equation itself. In particular this should be true with equation (5.2.18) who depends only on $q_{j}$ and $W_{j}$. Hence it is in considering new equations such as these where we could benefit from separable coordinates.

To take this idea further we can ask what if multiple coordinates were separable? Would this give more equations such as (5.2.18) that would help the solving for $S$ ? To bring in a further separable coordinate $q_{k}$, we continue from the point of obtaining the equations (5.2.18) and (5.2.19). However now, we treat (5.2.19) as a new Hamilton-Jacobi equation with $q_{k}$ as its separable coordinate. We also extend on equation (5.2.14) and require that it is in fact of the form

$$
S(q ; E, \beta ; t)=W_{j}\left(q_{j} ; E, \beta\right)+W_{k}\left(q_{k} ; E, \beta\right)+\tilde{W}\left(q_{l} ; E, \beta\right)-E t, \quad W_{k} \in C^{2}(\mathbb{R})
$$

and also ask that the equation (5.2.19) may be rearranged to be of the form

$$
f_{k}\left(q_{k} ; \frac{\partial W_{k}}{\partial q_{k}} ; E, \beta\right)=g_{k}\left(q_{l} ; \frac{\partial \tilde{W}}{\partial q_{l}} ; E, \beta\right) \text {. }
$$

By $q_{l}$ we now denote all coordinates other than $q_{j}$ and $q_{k}$. We may then repeat a similar argument to before that either side of the equation (5.2.21) may vary independently. Hence equation (5.2.21) can only be true if either side is equal to the same constant, i.e.

$$
f_{k}\left(q_{k} ; \frac{\partial W_{k}}{\partial q_{k}} ; E, \beta\right)=\beta_{k}
$$

and

$$
g_{k}\left(q_{l} ; \frac{\partial \tilde{W}}{\partial q_{l}} ; E, \beta\right)=\beta_{k} .
$$

Hence in (5.2.22) we now have a further equation that is simpler to solve which in this case is for $W_{k}$. 
In the most ideal situation, we would like to iterate this method a further $n-3$ times so that we obtain more simpler equations such as (5.2.22). Overall to do this, we would require that equation (5.2.14) to be of the form

$$
S(q ; E, \beta ; t)=\sum_{l=1}^{n} W_{l}\left(q_{l} ; E, \beta\right)-E t
$$

and that each function $g_{m}, m=2, \ldots, n$, allows for the necessary rearrangement so that equations such as (5.2.22) and (5.2.23) may be obtained at each stage of the iterative method. Eventually we would leave $n$ equations to solve:

$$
f_{1}\left(q_{1} ; \frac{\partial W_{1}}{\partial q_{1}} ; E, \beta\right)=E
$$

and

$$
f_{m}\left(q_{m} ; \frac{\partial W_{m}}{\partial q_{m}} ; E, \beta\right)=\beta_{m}, \quad m=2, \ldots, n .
$$

Clearly by this point all the desired constants $E, \beta_{2}, \ldots, \beta_{m}$ have been introduced to the solutions. We now arguaby have $n$ simpler equations to solve and for only one of the $W_{j}$ in each case. As said before this should be easier than solving the Hamilton-Jacobi equation itself. We will see that this is indeed the case when we opt to us this approach with examples to follow in the next chapter.

In the context of the above equations (5.2.25) and (5.2.26), we refer to the constants $E, \beta_{2}, \ldots, \beta_{m}$ as the separation constants. Should a problem allow us to obtain the $n$ equations at (5.2.25) and (5.2.26) we say that the associated Hamilton-Jacobi equation is completely separable.

A final discussion we present about separable coordinates is their relation to cyclic coordinartes. With the help of [26, Chapter 10.5] we find that the form of Hamilton's principal function is changed when the Hamilton function has coordinates who are cyclic:

Proposition 5.2.5. Given a Hamilton function suppose that $q_{j}$ is cyclic. Further let Hamilton's principal function $S$ be as at (5.2.14). Then it may be rewritten as

$$
S(q ; E, \beta ; t)=\tilde{W}\left(q_{l} ; E, \beta\right)+\beta_{j} q_{j}-E t
$$

where $\beta_{j}$ is the conserved value of the coordinate $p_{j}$.

Proof. To begin we know from Theorem 3.3.5 in Section 3.3 that $q_{j}$ being cyclic implies that $p_{j}$ is constant. Let us call this constant $\beta_{j}$. We also know from equation (5.2.2) (back when $S$ was first introduced) that $S$ satisfies

$$
\frac{\partial S}{\partial q_{j}}=p_{j}
$$


However using that $S$ has the form of (5.2.14) with the fact that $p_{j}$ is constant, equation (5.2.28) may be rewritten as

$$
\frac{\partial W_{j}}{\partial q_{j}}=\beta_{j} .
$$

However, equation (5.2.29) now tells us more about $W_{j}$. Integration of (5.2.29) gives

$$
W_{j}\left(q_{j} ; E, \beta\right)=\beta_{j} q_{j} .
$$

If we now substitute (5.2.30) into the form of $S$ at (5.2.14) we to obtain (5.2.27).

It is now clear from comparing Proposition 5.2.5 to Definiton 5.2.4 that should a coordinate be both separable and cyclic, then $S$ in fact appears as (5.2.27).

When working with examples in the next section, we will consider Hamilton functions who have cyclic coordinates. Hence the result of Propoisition 5.2.5 will be most useful. Additionally, we also want to give the best situation for solving the associated Hamilton-Jacobi equation of a given a problem, i.e. we would ideally want to solve $n$ equation such as (5.2.25) and (5.2.26) when trying to find $S$. Therefore we will always assume that Hamilton's principal function has the form (5.2.24). However, as already mentioned some of these examples will have a cyclic coordinate, say $q_{j}$. Using Proposition 5.2.5 the form of $S$ in this situation will in fact appear as

$$
S(q ; E, \beta ; t)=\sum_{\substack{l=1 \\ l \neq j}}^{n} W_{l}\left(q_{l} ; E, \beta\right)+\beta_{j} q_{j}-E t .
$$

So to conlcude, given a problem with a cyclic coordinate $q_{j}$, we will opt to use (5.2.31) as $S$ to give ourselves the best situation for solving the problem. 


\section{Chapter 6}

\section{Examples Using the Hamilton-Jacobi Theory}

Using the theory from the previous chapter, we study the examples discussed in Chapter 4 . We see that this provides an alternative way in studying the dynamics, and in some cases arguably a quicker and more efficient way of doing so. As before we consider examples corresponding to the Harmonic Oscillator, the Kepler problem, and the relativistic dynamics. Comparing back to Chapter 4 we of course would expect similar results. In the coming Section 6.3 we use the theory of HamiltonJacobi to present an additional example. It ties with the relativistic dynamics and generalises the problem by introducing a fractional power.

An elegance in the Hamilton-Jacobi approach is that it allows us to work through many of the examples independent of an explicit potential. Instead we adopt $V(q)$ as the potential. We take advantange of this for the Harmonic Oscillator and the Kepler problem in Section 6.1 where we accumulate both cases into one discussion using a general potential $V$. These examples act as generalisations of the Harmonic Oscillator and the Kepler problem to how we have seen them before. However taking the appropriate potential gains back the specific solutions for each case.

Our approach for studying the examples follows Section 5.2 from the previous chapter: given a Hamilton function $\mathcal{H}(q, p)$ and its Hamilton's equations to solve, we solve instead the associated Hamilton-Jacobi equation to find the generating function. We know that this function induces a canonical transformation $(q, p) \mapsto$ $(Q, P)$ such that the transformed coordinates $Q$ and $P$ are constant. Using this with the generating function derivatives we are then able to solve for the coordinates $q$ and $p$ as solutions of Hamilton's equations. Looking back to Chapter 4 we would see that many of the examples contain cyclic coordinates. Hence the concluding discussion of Secton 5.2 about separable coordinates will be most useful in finding the generating function. 
For all the examples within a specific dimension, i.e. the collection of examples for when either $n=1,2$ or 3, the method used for the examples is quite similar each time. Solving the example for the Harmonic Oscillator in two-dimensions follows a similar approach to that used when considering the two-dimensioanl example for the relativistic dynamics for instance. Hence to avoid repetition of the arguments we only show the full method for one example within each dimension. For the Harmonic Oscillator and Kepler problem in Section 6.1, the cases for when $n=1$ and $n=3$ are fully demonstrated where in Section 6.2 with the relativistic dynamics, only the case when $n=2$ has its full workings. For the remainder of the examples we only collect the main results with the understanding that they can be fully worked out analogously to those who are fully demonstrated.

As before conditions will have to be taken so that our results are well-defined. In most case they will follow as they did in Chapter 4 and so in this case they will not be repeated here and we refer back.

\subsection{Harmonic Oscillator and Kepler Problem (Hamilton-Jacobi Approach)}

Here are the examples for the Harmonic Oscillator and the Kepler problem now studied using the Hamilton-Jacobi theory from Section 5.2. As in Chapter 4 we begin with the one-dimensional example followed by the two and three-dimensional cases. We will see that most of the work can be carried out using a general potential term $V(q)$. Should we want to specialise and express explicit solutions we can employ the potentials

$$
V(q)=\frac{1}{\alpha^{*}}\|q\|^{\alpha^{*}}, \quad \alpha^{*}=\frac{\alpha}{\alpha-1} \quad \text { and } \quad V(q)=-\gamma_{n, \alpha}\|q\|^{\alpha-n}, q \neq 0,
$$

for the Harmonic Oscillator and the Kepler problem respectively. The constant $\gamma_{n, \alpha}$ was defined at the beginning of Section 4.2.

Example 6.1.1. We want to study the dynamics associated with $\mathcal{H} \in C^{1}\left(\mathbb{R}^{2}\right)$ such that

$$
\mathcal{H}(q, p)=\frac{1}{\alpha}|p|^{\alpha}+V(q), \quad 1<\alpha \leq 2 .
$$

We take the approach of the Hamilton-Jacobi theory and solve the corresponding Hamilton-Jacobi equation to find the generating function $S(q, P, t)$ who we know by (5.2.9) has the form

$$
S(q, E, t)=W(q, E)-E t .
$$

As before $E$ is the conserved value of (6.1.2) determined by the initial conditions, i.e. $\mathcal{H}(q(t), p(t))=\mathcal{H}\left(q_{0}, p_{0}\right)=E$. We know that $S$ induces a canonical transformation 
$(q, p) \mapsto(Q, P)$ such that the transformed coordinates are constant:

$$
Q=\gamma, \quad P=E,
$$

where we recall from (5.2.10) how the coordinate $P$ was identified. Once having solved for $S$ we then use the conserved coordinates at (6.1.4) with the associated generating function derivatives (recall (5.2.11))

$$
p=\frac{\partial S}{\partial q} \quad \text { and } \quad Q=\frac{\partial S}{\partial P}
$$

to solve for $q$ and $p$.

We begin by solving the following Hamilton-Jacobi equation for $W$ to find $S$ :

$$
\mathcal{H}\left(q, \frac{\partial W}{\partial q}\right)=\frac{1}{\alpha}\left|\frac{\partial W}{\partial q}\right|^{\alpha}+V(q)=E .
$$

As with some previous one-dimensional examples, i.e. Examples 4.1.1 and 4.2.1, we consider four distinct cases for the solutions to deal with the absolute values above. This once again gives a symmetry property of sorts in our results. The four individual cases follow as $q \geq 0$ and $\frac{\partial W}{\partial q} \geq 0, q \geq 0$ and $\frac{\partial W}{\partial q}<0, q<0$ and $\frac{\partial W}{\partial q} \geq 0$, and $q<0$ and $\frac{\partial W}{\partial q}<0$. We solve (6.1.6) only for the first case, however the method may then be carried out analogously for the remaining three cases. Having solved for $q \geq 0$ and $\frac{\partial W}{\partial q} \geq 0$, we then give a solution that collects what we would have overall for all cases.

Let $q \geq 0$ and $\frac{\partial W}{\partial q} \geq 0$. Then the Hamilton-Jacobi equation at (6.1.6) appears as

$$
\mathcal{H}\left(q, \frac{\partial W}{\partial q}\right)=\frac{1}{\alpha}\left(\frac{\partial W}{\partial q}\right)^{\alpha}+V(q)=E .
$$

Rearranging (6.1.7) gives

$$
\frac{\partial W}{\partial q}=\alpha^{\frac{1}{\alpha}}(E-V(q))^{\frac{1}{\alpha}}
$$

determining Hamilton's characteristic function as

$$
W(q, E)=\alpha^{\frac{1}{\alpha}} \int(E-V(q))^{\frac{1}{\alpha}} \mathrm{d} q .
$$

Hence by (6.1.3) Hamilton's principal function follows as

$$
S(q, E, t)=\alpha^{\frac{1}{\alpha}} \int(E-V(q))^{\frac{1}{\alpha}} \mathrm{d} q-E t .
$$


Now that we have the generating function we may use its derivatives at (6.1.5) to determine $q$ and $p$. By the first equation at (6.1.5) we have

$$
p=\alpha^{\frac{1}{\alpha}}(E-V(q))^{\frac{1}{\alpha}} .
$$

The second equation of (6.1.5) then gives

$$
\gamma=\frac{\partial S}{\partial E}=\frac{\partial W}{\partial E}-t
$$

or

$$
\gamma+t=\alpha^{\frac{1-\alpha}{\alpha}} \int(E-V(q))^{\frac{1-\alpha}{\alpha}} \mathrm{d} q .
$$

Let us introduce the more specific potentials from (6.1.1). We begin with the Harmonic Oscillator and set in (6.1.7)

$$
V(q)=\frac{1}{\alpha^{*}} q^{\alpha^{*}}, \quad \alpha^{*}=\frac{\alpha}{\alpha-1}, \quad q \geq 0 .
$$

Through this equations (6.1.10) and (6.1.11) become

$$
p=\alpha^{\frac{1}{\alpha}}\left(E-\frac{1}{\alpha^{*}} q^{\alpha^{*}}\right)^{\frac{1}{\alpha}}
$$

and

$$
\begin{aligned}
\gamma+t & =\alpha^{\frac{1-\alpha}{\alpha}} \int\left(E-\frac{1}{\alpha^{*}} q^{\alpha^{*}}\right)^{\frac{1-\alpha}{\alpha}} \mathrm{d} q \\
& =(\alpha E)^{-\frac{1}{\alpha^{*}}} q_{2} F_{1}\left(\frac{\alpha-1}{\alpha}, \frac{\alpha-1}{\alpha} ; \frac{2 \alpha-1}{\alpha} ; \frac{q^{\alpha^{*}}}{\alpha^{*} E}\right) .
\end{aligned}
$$

Incorporating the initial conditions, the above would give the same solutions as at (4.1.6) from the corresponding one-dimensional example in Section 4.1. Through this the additional constant $\gamma$ can then be determined in terms of the initial values and E. A clearer comparison is equation (B.9) from the calculations in Appendix $B$. Using our solution of $q$ with (6.1.13) would then determine $p$.

For the Kepler problem we take in the Hamilton-Jacobi equation (6.1.7) the potential to be

$$
V(q)=-\gamma_{1, \alpha} q^{\alpha-1}, \quad q \neq 0 .
$$

Through this equations (6.1.10) and (6.1.11) appear as

$$
p=\alpha^{\frac{1}{\alpha}}\left(E+\gamma_{1, \alpha} q^{\alpha-1}\right)^{\frac{1}{\alpha}}
$$


and

$$
\begin{aligned}
\gamma+t & =\alpha^{\frac{1-\alpha}{\alpha}} \int\left(E+\gamma_{1, \alpha} q^{\alpha-1}\right)^{\frac{1-\alpha}{\alpha}} \mathrm{d} q \\
& =\alpha^{\frac{1-3 \alpha}{\alpha}} E^{\frac{1-2 \alpha}{\alpha}} \times\left(\alpha^{2} E q_{2} F_{1}\left(\frac{1}{\alpha-1}, \frac{-1}{\alpha} ; \frac{\alpha}{\alpha-1} ; \frac{-\tilde{\gamma}_{1, \alpha} q^{\alpha-1}}{\alpha E}\right)\right. \\
& \left.-\tilde{\gamma}_{1, \alpha} q^{\alpha}{ }_{2} F_{1}\left(\frac{\alpha-1}{\alpha}, \frac{\alpha}{\alpha-1} ; \frac{2 \alpha-1}{\alpha-1} ; \frac{-\tilde{\gamma}_{1, \alpha} q^{\alpha-1}}{\alpha E}\right)\right)
\end{aligned}
$$

respectively. Incorporating the initial conditions once again, the above would give the same solution as at (4.2.13) from the corresponding one-dimensional example in Section 4.2. As before the additional constant $\gamma$ can then be determined in terms of the initial values and $E$. Using our solution of $q$ with (6.1.16), we can also determine $p$.

Taking into account the remaining cases for the signs of $q$ and $\frac{\partial W}{\partial q}$, we now collect what we would have overall by following the method above for each case. The solution to the Hamilton-Jacobi equation at (6.1.6) appears as

$$
S(q, E, t)= \pm \alpha^{\frac{1}{\alpha}} \int(E-V(q))^{\frac{1}{\alpha}} \mathrm{d} q-E t
$$

where $V(q)=\frac{1}{\alpha^{*}}|q|^{\alpha^{*}}$ for the Harmonic Oscillator and $V(q)=-\gamma_{1, \alpha}|q|^{\alpha-1}, q \neq 0$, for the Kepler problem. In the case of the Harmonic Oscillator the solutions for $p$ and $q$ are determined by

$$
p= \pm \alpha^{\frac{1}{\alpha}}\left(E-\frac{1}{\alpha^{*}}|q|^{\alpha^{*}}\right)^{\frac{1}{\alpha}}
$$

and

$$
\gamma+t= \pm(\alpha E)^{-\frac{1}{\alpha^{*}}} q_{2} F_{1}\left(\frac{\alpha-1}{\alpha}, \frac{\alpha-1}{\alpha} ; \frac{2 \alpha-1}{\alpha} ; \frac{|q|^{\alpha^{*}}}{\alpha^{*} E}\right)
$$

repectively. Similarly for the Kepler problem the solutions $p$ and $q$ are given by

$$
p= \pm \alpha^{\frac{1}{\alpha}}\left(E+\gamma_{1, \alpha}|q|^{\alpha-1}\right)^{\frac{1}{\alpha}}
$$

and

$$
\begin{aligned}
\gamma+t & = \pm \alpha^{\frac{1-3 \alpha}{\alpha}} E^{\frac{1-2 \alpha}{\alpha}} \\
& \times\left(\alpha^{2} E|q|_{2} F_{1}\left(\frac{1}{\alpha-1}, \frac{-1}{\alpha} ; \frac{\alpha}{\alpha-1} ; \frac{-\tilde{\gamma}_{1, \alpha}|q|^{\alpha-1}}{\alpha E}\right)\right.
\end{aligned}
$$




$$
\left.-\tilde{\gamma}_{1, \alpha}|q|_{2}^{\alpha} F_{1}\left(\frac{\alpha-1}{\alpha}, \frac{\alpha}{\alpha-1} ; \frac{2 \alpha-1}{\alpha-1} ; \frac{-\tilde{\gamma}_{1, \alpha}|q|^{\alpha-1}}{\alpha E}\right)\right)
$$

respectively. The sign coefficients in the above are determined by which individual case for the signs of $q$ and $\frac{\partial W}{\partial q}$ is considered. Constants such as $\gamma$ may be eliminated using initial values as was done earlier for when $q \geq 0$ and $\frac{\partial W}{\partial q} \geq 0$.

For both the Harmonic Oscillator and the Kepler problem we have already solved for their solutions in Sections 4.1 and 4.2. Hence conditions for ensuring that the work here is well-defined will be the same to what was discussed before at these sections. Therefore we do not repeat them here.

Example 6.1.2. The Hamilton function whose dynamics we want to study is $\mathcal{H} \in$ $C^{1}\left(\mathbb{R}^{4}\right)$ such that

$$
\mathcal{H}(q, p)=\frac{1}{\alpha}\|p\|^{\alpha}+V(q), \quad 1<\alpha \leq 2 .
$$

Similar to before with the two-dimensional examples at Chapter 4, we employ polar coordinates to obtain an equivalent form to (6.1.18). This is given by $\mathcal{H}_{2} \in$ $C^{1}((0, \infty) \times[0,2 \pi) \times \mathbb{R} \backslash\{0\} \times \mathbb{R})$ such that

$$
\mathcal{H}_{2}\left(r, \vartheta, p_{r}, p_{\vartheta}\right)=\frac{1}{\alpha}\left(p_{r}^{2}+\frac{p_{\vartheta}^{2}}{r^{2}}\right)^{\frac{\alpha}{2}}+V(r) .
$$

Using the Hamilton-Jacobi theory we solve the Hamilton-Jacobi equation to obtain a generating function that induces a canonical transformation such that the transformed Hamilton function is zero and the transformed coordinates are constant:

$$
Q_{r}=\gamma_{1}, \quad Q_{\vartheta}=\gamma_{2}, \quad P_{r}=E, \quad P_{\vartheta}=\beta_{2} .
$$

In the above $E$ is the conserved value of the Hamilton function (6.1.19) determined by the initial conditions. Using the conserved coordinates at (6.1.20) with the generating function derivatives the solutions to the associated Hamilton's equations of (6.1.19) are found to satisfy

$$
\begin{gathered}
\gamma_{1}+t= \pm \alpha^{\frac{2-\alpha}{\alpha}} \int \frac{r(E-V(r))^{\frac{2-\alpha}{\alpha}}}{\sqrt{\alpha^{\frac{\alpha}{2}} r^{2}(E-V(r))^{\frac{2}{\alpha}}-\beta_{2}^{2}}} \mathrm{~d} r, \\
\gamma_{2} \pm \int \frac{\beta_{2}}{r \sqrt{\alpha^{\frac{2}{\alpha}} r^{2}(E-V(r))^{\frac{2}{\alpha}}-\beta_{2}^{2}}} \mathrm{~d} r=\vartheta, \\
p_{r}= \pm \sqrt{\alpha^{\frac{2}{\alpha}}(E-V(r))^{\frac{2}{\alpha}}-\frac{\beta_{2}^{2}}{r^{2}}}
\end{gathered}
$$


and

$$
p_{\vartheta}=\beta_{2},
$$

where $\gamma_{i}, i=1,2$, are constants of integration who may be determinied using the initial conditions.

The solutions may be completely determined with the implementation of the initial conditions $q\left(t_{0}\right)=q_{0}$ and $p\left(t_{0}\right)=p_{0}$. For a further presentation of the work behind this example we refer to the later Example 6.2.2 which shows the twodimensional example associated to the relatvistic dynamics. Following the method here, the full workings for this example can be computed.

There are some conditions we need to impose such that the solutions are well-defined. Following the more detailed Example 4.1.2 we can deduce similarly that $p_{r} \neq 0$ is sufficient.

Taking $V(q)=\frac{1}{\alpha^{*}}\|q\|^{\alpha^{*}}$ in (6.1.21) we obtain the solution (4.1.26) from the corresponding two-dimensional Harmonic Oscillator example in Section 4.1. Similarly taking $V(q)=-\gamma_{2, \alpha}\|q\|^{\alpha-2}, q \neq 0$, gives (4.2.28) as seen in the two-dimensional Kepler problem example from Section 4.2.

Next follows the extension of Example 6.1.2 to three-dimensions:

Example 6.1.3. The Hamilton function whose dynamics we want to study is $\mathcal{H} \in$ $C^{1}\left(\mathbb{R}^{6}\right)$ such that

$$
\mathcal{H}(q, p)=\frac{1}{\alpha}\|p\|^{\alpha}+V(q), \quad 1<\alpha \leq 2 .
$$

We employ spherical polar coordinates to obtain an equivalent form to (6.1.25) given by $\mathcal{H}_{3} \in C^{1}\left((0, \infty) \times[0, \pi] \times[0,2 \pi) \times \mathbb{R} \backslash\{0\} \times \mathbb{R}^{2}\right)$ such that

$$
\mathcal{H}_{3}\left(r, \phi, \theta, p_{r}, p_{\phi}, p_{\theta}\right)=\frac{1}{\alpha}\left(p_{r}^{2}+\frac{p_{\phi}^{2}}{r^{2}}+\frac{p_{\theta}^{2}}{r^{2} \sin ^{2} \phi}\right)^{\frac{\alpha}{2}}+V(r) .
$$

Using the Hamilton-Jacobi theory from Section 5.2, we want to solve the corresponding Hamilton-Jacobi equation to (6.1.25) to find the generating function $S$. We know from (5.2.9) that $S$ has the form

$$
S\left(r, \phi, \theta ; E, \beta_{2}, \beta_{3} ; t\right)=W\left(r, \phi, \theta ; E, \beta_{2}, \beta_{3}\right)-E t .
$$

Note $E$ is the conserved value of the Hamilton function (6.1.25) determined by the initial conditions $r\left(t_{0}\right)=r^{0}, \phi\left(t_{0}\right)=\phi^{0}, \theta\left(t_{0}\right)=\theta^{0}, p_{r}\left(t_{0}\right)=p_{r}^{0}, p_{\phi}\left(t_{0}\right)=p_{\phi}^{0}$ and $p_{\theta}\left(t_{0}\right)=p_{\theta}^{0}$, i.e.

$$
\mathcal{H}_{3}\left(r(t), \phi(t), \theta(t), p_{r}(t), p_{\phi}(t), p_{\theta}(t)\right)=\mathcal{H}_{3}\left(r^{0}, \phi^{0}, \theta^{0}, p_{r}^{0}, p_{\phi}^{0}, p_{\theta}^{0}\right)=E>0 .
$$


The constants $\beta_{2}$ and $\beta_{3}$ are constants of integration to be determined.

As before, we know that the function $S$ induces a canonical transformation $\left(r, \phi, \theta, p_{r}, p_{\phi}, p_{\theta}\right) \mapsto\left(Q_{r}, Q_{\phi}, Q_{\theta}, P_{r}, P_{\phi}, P_{\theta}\right)$ such that the transformed Hamilton function is zero and the transformed coordinates are constant:

$$
Q_{r}=\gamma_{1}, \quad Q_{\phi}=\gamma_{2}, \quad Q_{\theta}=\gamma_{3}, \quad P_{r}=E, \quad P_{\phi}=\beta_{2}, \quad P_{\theta}=\beta_{3} .
$$

To form (6.1.28) we recall (5.2.10) where $P_{r}, P_{\phi}$ and $P_{\theta}$ may identified as they are above.

Once having solved for $S$, we can use the conserved coordinates at (6.1.28) with the generating function derivatives

$$
\begin{gathered}
p_{r}=\frac{\partial S}{\partial r}, \quad p_{\phi}=\frac{\partial S}{\partial \phi}, \quad p_{\theta}=\frac{\partial S}{\partial \theta}, \\
Q_{r}=\frac{\partial S}{\partial P_{r}}, \quad Q_{\phi}=\frac{\partial S}{\partial P_{\phi}}, \quad Q_{\theta}=\frac{\partial S}{\partial P_{\theta}},
\end{gathered}
$$

to solve for $r, \phi, \theta, p_{r}, p_{\phi}$ and $p_{\theta}$. The Hamilton-Jacobi equation appears as

$$
\begin{aligned}
\mathcal{H}_{3} & \left(r, \phi, \theta, \frac{\partial W}{\partial r}, \frac{\partial W}{\partial \phi}, \frac{\partial W}{\partial \theta}\right) \\
& =\frac{1}{\alpha}\left(\left(\frac{\partial W}{\partial r}\right)^{2}+\frac{1}{r^{2}}\left(\frac{\partial W}{\partial \phi}\right)^{2}+\frac{1}{r^{2} \sin ^{2} \phi}\left(\frac{\partial W}{\partial \theta}\right)^{2}\right)^{\frac{\alpha}{2}}+V(r)=E .
\end{aligned}
$$

Looking at (6.1.31), it is evident that solving for $W$ (and hence $S$ ) is not so obvious. Hence to solve (6.1.31) we follow the study of separable coordinates presented towards the end of Section 5.2. We begin by adopting a different form for $S$ as suggested by (5.2.24). We set

$$
S\left(r, \phi, \theta ; E, \beta_{2}, \beta_{3} ; t\right)=W_{r}\left(r ; E, \beta_{2}, \beta_{3}\right)+W_{\phi}\left(\phi ; E, \beta_{2}, \beta_{3}\right)+W_{\theta}\left(\theta ; E, \beta_{2}, \beta_{3}\right)-E t
$$

for $W_{r} \in C^{2}((0, \infty)), W_{\phi} \in C^{2}([0, \pi]), W_{\theta} \in C^{2}([0,2 \pi))$. We make a further useful observation: note that $\theta$ is a cyclic coordinate in (6.1.26). It was also discussed in Section 5.2 how cyclic coordinates can alter the form of $S$ to help the solving and so we follow (5.2.31) and rewrite (6.1.32) as

$$
S\left(r, \phi, \theta, E, \beta_{2}, \beta_{3}, t\right)=W_{r}\left(r ; E, \beta_{2}, \beta_{3}\right)+W_{\phi}\left(\phi ; E, \beta_{2}, \beta_{3}\right)+\beta_{3} \theta-E t
$$

where $\beta_{3}$ is the conserved coordinate $p_{\theta}$ solving

$$
\beta_{3}=\frac{\partial W_{\theta}}{\partial \theta}, \quad W_{\theta}\left(\theta, \beta_{3}\right):=\beta_{3} \theta
$$


The generating function derivatives at (6.1.29) and (6.1.30) as well as the HamiltonJacobi equation at (6.1.31) now appear as

$$
\begin{gathered}
p_{r}=\frac{\partial W_{r}}{\partial r}, \quad p_{\phi}=\frac{\partial W_{\phi}}{\partial \phi}, \quad p_{\theta}=\frac{\partial W_{\theta}}{\partial \vartheta} \\
Q_{r}=\frac{\partial W_{r}}{\partial E}+\frac{\partial W_{\phi}}{\partial E}-t, \quad Q_{\phi}=\frac{\partial W_{r}}{\partial \beta_{2}}+\frac{\partial W_{\phi}}{\partial \beta_{2}}, \quad Q_{\theta}=\frac{\partial W_{r}}{\partial \beta_{3}}+\frac{\partial W_{\phi}}{\partial \beta_{3}}+\theta
\end{gathered}
$$

and

$$
\frac{1}{\alpha}\left(\left(\frac{\partial W_{r}}{\partial r}\right)^{2}+\frac{1}{r^{2}}\left(\frac{\partial W_{\phi}}{\partial \phi}\right)^{2}+\frac{\beta_{3}^{2}}{r^{2} \sin ^{2} \phi}\right)^{\frac{\alpha}{2}}+V(r)=E
$$

respectively. We can rearrange (6.1.37) to have

$$
\beta_{3}= \pm r \sin \phi \sqrt{\alpha^{\frac{2}{\alpha}}(E-V(r))^{\frac{2}{\alpha}}-\left(\frac{\partial W_{r}}{\partial r}\right)^{2}-\frac{1}{r^{2}}\left(\frac{\partial W_{\phi}}{\partial \phi}\right)^{2}} .
$$

Defining $f_{3}\left(\frac{\partial W_{\theta}}{\partial \theta}\right)$ and $g_{3}\left(r, \phi, \frac{\partial W_{r}}{\partial r}, \frac{\partial W_{\phi}}{\partial \phi}, E\right)$ as the left and right-hand sides of (6.1.38) respectively we have the form of (5.2.17). Hence we can argue as was done there to deduce that

$$
f_{3}\left(\frac{\partial W_{\theta}}{\partial \theta}\right)=\beta_{3}
$$

and

$$
g_{3}\left(r, \phi, \frac{\partial W_{r}}{\partial r}, \frac{\partial W_{\phi}}{\partial \phi}, E\right)=\beta_{3} .
$$

Therefore by Definition 5.2.4 the coordinate $\theta$ is separable and $\beta_{3}$ is a separation constant.

We may find $\phi$ as a further separable coordinate if we follow the method suggested in Section 5.2 and now read equation (6.1.39) as a new Hamilton-Jacobi equation. Rearranging (6.1.39) we have

$$
\pm \sqrt{\left(\frac{\partial W_{\phi}}{\partial \phi}\right)^{2}+\frac{\beta_{3}^{2}}{\sin ^{2} \phi}}= \pm r \sqrt{\alpha^{\frac{2}{\alpha}}(E-V(r))^{\frac{2}{\alpha}}-\left(\frac{\partial W_{r}}{\partial r}\right)^{2}} .
$$

Defining $f_{2}\left(\phi, \frac{\partial W_{\phi}}{\partial \theta}\right)$ and $g_{2}\left(r, \frac{\partial W_{r}}{\partial r}, E\right)$ as the left and right-hand sides of (6.1.40) respectively we once again have the form of (5.2.17). We can argue in a similar manner to deduce that

$$
f_{2}\left(\phi, \frac{\partial W_{\phi}}{\partial \theta}\right)=\beta_{2}
$$


and

$$
g_{2}\left(r, \frac{\partial W_{r}}{\partial r}, E\right)=\beta_{2} .
$$

Hence by Definition 5.2.4 this confirms $\phi$ as also separable and $\beta_{2}$ a further separation constant.

Rearranging (6.1.41) and (6.1.42) we can solve $\frac{\partial W_{r}}{\partial r}$ and $\frac{\partial W_{\phi}}{\partial \theta}$ to be

$$
W_{r}= \pm \int \sqrt{\alpha^{\frac{2}{\alpha}}(E-V(r))^{\frac{2}{\alpha}}-\frac{\beta_{2}^{2}}{r^{2}}} \mathrm{~d} r
$$

and

$$
W_{\phi}= \pm \int \sqrt{\beta_{2}^{2}-\frac{\beta_{3}^{2}}{\sin ^{2} \phi}} \mathrm{d} \phi
$$

respectively. Therefore using (6.1.33) Hamilton's principal function appears as

$$
\begin{aligned}
S\left(r, \phi, \theta ; E, \beta_{2}, \beta_{3} ; t\right) & = \pm \int \sqrt{\alpha^{\frac{2}{\alpha}}(E-V(r))^{\frac{2}{\alpha}}-\frac{\beta_{2}^{2}}{r^{2}}} \mathrm{~d} r \\
& \pm \int \sqrt{\beta_{2}^{2}-\frac{\beta_{3}^{2}}{\sin ^{2} \phi}} \mathrm{d} \phi+\beta_{3} \theta-E t
\end{aligned}
$$

Using the derivatives from (6.1.35) we find $p_{r}$ and $p_{\phi}$ to be

$$
p_{r}=\frac{\partial S}{\partial r}=\frac{\partial W_{r}}{\partial r}= \pm \sqrt{\alpha^{\frac{2}{\alpha}}(E-V(r))^{\frac{2}{\alpha}}-\frac{\beta_{2}^{2}}{r^{2}}}
$$

and

$$
p_{\phi}=\frac{\partial S}{\partial \phi}=\frac{\partial W_{\phi}}{\partial \phi}= \pm \sqrt{\beta_{2}^{2}-\frac{\beta_{3}^{2}}{\sin ^{2} \phi}},
$$

respectively, as well as the coordinate $p_{\theta}$ to be already what we know:

$$
p_{\theta}=\frac{\partial W_{\theta}}{\partial \theta}=\beta_{3}
$$

For the coordinates $r, \phi$ and $\theta$, we look to the derivatives from (6.1.36) to find that they satisfy firstly

or

$$
\gamma_{1}=Q_{1}=\frac{\partial S}{\partial E}=\frac{\partial W_{r}}{\partial E}+\frac{\partial W_{\phi}}{\partial E}-t
$$

$$
t+\gamma_{1}= \pm \alpha^{\frac{2-\alpha}{\alpha}} \int \frac{r(E-V(r))^{\frac{2-\alpha}{\alpha}}}{\sqrt{\alpha^{\frac{2}{\alpha}} r^{2}(E-V(r))^{\frac{2}{\alpha}}-\beta_{2}^{2}}} \mathrm{~d} r
$$


secondly

$$
\gamma_{2}=Q_{2}=\frac{\partial S}{\partial \beta_{2}}=\frac{\partial W_{r}}{\partial \beta_{2}}+\frac{\partial W_{\phi}}{\partial \beta_{2}}
$$

or

$$
\gamma_{2} \pm \int \frac{\beta_{2}}{r \sqrt{\alpha^{\frac{2}{\alpha}} r^{2}(E-V(r))^{\frac{2}{\alpha}}-\beta_{2}^{2}}} \mathrm{~d} r= \pm \int \frac{\beta_{2} \sin \phi}{\sqrt{\beta_{2}^{2} \sin ^{2} \phi-\beta_{3}^{2}}} \mathrm{~d} \phi,
$$

and thirdly

$$
\gamma_{3}=Q_{3}=\frac{\partial S}{\partial \beta_{3}}=\frac{\partial W_{r}}{\partial \beta_{3}}+\frac{\partial W_{\phi}}{\partial \beta_{3}}+\theta
$$

or

$$
\gamma_{3} \pm \int \frac{\beta_{3}}{\sin \phi \sqrt{\beta_{2}^{2} \sin ^{2} \phi-\beta_{3}^{2}}} \mathrm{~d} \phi=\theta
$$

Assuming the integration is possible, equation (6.1.48) can be used to determine $r$ as a function of $t$. This can then be used with (6.1.49) to find $\phi$ similarly. Finally both solutions for $r$ and $\phi$ can be used with (6.1.50) to find $\theta$. As before initial conditions may be employed to eliminate the constants $\gamma_{i}, i=1,2,3$, giving them as functions of the initial values and $E$.

There are some conditions we need to impose such that everything is well-defined:

(i) $E-V(r)>0$,

(ii) $\alpha^{\frac{2}{\alpha}} r^{2}(E-V(r))^{\frac{2}{\alpha}}-\beta_{2}^{2}>0$,

(iii) $\beta_{2}^{2}-\frac{\beta_{3}^{2}}{\sin ^{2} \phi}>0$.

Following the method seen at Example 4.1 .3 we deduce that $p_{r} \neq 0$ is sufficient for the above to hold.

Taking $V(q)=\frac{1}{\alpha^{*}}\|q\|^{\alpha^{*}}$ in (6.1.48) we obtain the solution (4.1.45) from the corresponding three-dimensional Harmonic Oscillator example in Section 4.1. Similarly taking $V(q)=-\gamma_{3, \alpha}\|q\|^{\alpha-3}, q \neq 0$, gives (4.2.45) as seen in three-dimensional Kepler problem example from Section 4.2. 


\subsection{Relativistic Dynamics (Hamilton-Jacobi Approach)}

Here we present the examples associated with the relativistic dynamics but now using the Hamilton-Jacobi theory from Section 5.2. As before the one-dimensional case is considered followed by the two and three-dimensional cases. We work with a general potential $V(q)$.

Example 6.2.1. We want to study the dynamics associated with $\mathcal{H}_{1, R} \in C^{1}(\mathbb{R} \times$ $\mathbb{R} \backslash\{0\})$ such that

$$
\mathcal{H}_{1, R}(q, p)=\sqrt{m^{2}+p^{2}}-m+V(q) .
$$

Using the Hamilton-Jacobi theory we solve the Hamilton-Jacobi equation to obtain a generating function inducing a canonical transformation such that the transformed Hamilton function is zero and the transformed coordinates are constant:

$$
Q=\gamma, \quad P=E,
$$

where $E$ is the conserved value of the Hamilton function (6.2.1) determined by the initial conditions. Using the conserved coordinates at (6.2.2) with the generating function derivatives the solutions to the associated Hamilton's equations of (6.2.1) are found to satisfy

$$
p= \pm \sqrt{(E+m-V(q))^{2}-m^{2}}
$$

and

$$
\gamma+t= \pm \int \frac{E+m-V(q)}{\sqrt{(E+m-V(q))^{2}-m^{2}}} \mathrm{~d} q
$$

where $\gamma$ is a constant of integration that may be determined using the initial conditions.

Comparing (6.2.4) to (4.3.13) we see that we would obtain the same solution as that in the corresponding one-dimensional example covered before in Section 4.3. Using our solution of $q$ with (6.2.3) would then determine $p$. The solutions may be determined completely as in Example 6.1.1. In fact following this example one can illustrate the method used to obtain the solutions above in its fullest. A key distinction here however is that we need not consider four distinct cases for solving $q$ and p. When comparing back to Example 6.1.1 it is sufficient to follow only the first case covered there to deduce this example in its entirety.

There are some conditions we need to impose such that solutions are well-defined. Following the more detailed Example 4.1.2 we can deduce similarly that $p \neq 0$ is sufficient. 
Example 6.2.2. The Hamilton function whose dynamics we want to study is $\mathcal{H} \in$ $C^{1}\left(\mathbb{R}^{4}\right)$ such that

$$
\mathcal{H}(q, p)=\sqrt{m^{2}+\|p\|^{2}}-m+V(q) .
$$

We employ polar coordinates to obtain an equivalent form to (6.2.5) given by $\mathcal{H}_{2, R} \in$ $C^{1}((0, \infty) \times[0,2 \pi) \times \mathbb{R} \backslash\{0\} \times \mathbb{R})$ such that

$$
\mathcal{H}_{2, R}\left(r, \vartheta, p_{r}, p_{\vartheta}\right)=\sqrt{m^{2}+p_{r}^{2}+\frac{p_{\vartheta}^{2}}{r^{2}}}-m+V(r) .
$$

Using the Hamilton-Jacobi theory from Section 5.2, we want to solve the corresponding Hamilton-Jacobi equation to (6.2.6) to find the generating function $S$. We know from (5.2.9) that $S$ has the form

$$
S\left(r, \vartheta ; E, \beta_{2} ; t\right)=W\left(r, \vartheta ; E, \beta_{2}\right)-E t .
$$

Note $E$ is the conserved value of the Hamilton function (6.2.6) determined by the initial conditions $r\left(t_{0}\right)=r^{0}, \vartheta\left(t_{0}\right)=\vartheta^{0}, p_{r}\left(t_{0}\right)=p_{r}^{0}$ and $p_{\vartheta}\left(t_{0}\right)=p_{\vartheta}^{0}$, i.e.

$$
\mathcal{H}_{2, R}\left(r(t), \vartheta(t), p_{r}(t), p_{\vartheta}(t)\right)=\mathcal{H}_{2, R}\left(r^{0}, \vartheta^{0}, p_{r}^{0}, p_{\vartheta}^{0}\right)=E>0 .
$$

The constant $\beta_{2}$ is a constant of integration to be determined.

We know that the function $S$ induces a canonical transformation $\left(r, \vartheta, p_{r}, p_{\vartheta}\right) \mapsto$ $\left(Q_{r}, Q_{\vartheta}, P_{r}, P_{\vartheta}\right)$ such that the transformed Hamilton function is zero and the transformed coordinates are constant:

$$
Q_{r}=\gamma_{1}, \quad Q_{\vartheta}=\gamma_{2}, \quad P_{r}=E, \quad P_{\vartheta}=\beta_{2} .
$$

To form (6.2.8) we recall (5.2.10) where $P_{r}$ and $P_{\vartheta}$ may be identified as they are above.

Once having solved for $S$, we can use the conserved coordinates at (6.2.8) with the generating function derivatives

$$
\begin{gathered}
p_{r}=\frac{\partial S}{\partial r}, \quad p_{\vartheta}=\frac{\partial S}{\partial \vartheta}, \\
Q_{r}=\frac{\partial S}{\partial P_{r}}, \quad Q_{\vartheta}=\frac{\partial S}{\partial P_{\vartheta}}
\end{gathered}
$$

to solve for $r, \vartheta, p_{r}$ and $p_{\vartheta}$. The Hamilton-Jacobi equation appears as

$$
\mathcal{H}_{2, R}\left(r, \vartheta, \frac{\partial W}{\partial r}, \frac{\partial W}{\partial \vartheta}\right)=\sqrt{m^{2}+\left(\frac{\partial W}{\partial r}\right)^{2}+\frac{1}{r^{2}}\left(\frac{\partial W}{\partial \vartheta}\right)^{2}}-m+V(r)=E
$$

Looking at (6.2.11), it is evident that solving for $W$ (and hence $S$ ) is not so obvious. Hence to solve (6.2.11) we follow the study of separable coordinates presented towards 
the end of Section 5.2. We begin by adopting a different form for $S$ as suggested by (5.2.24). We set

$$
S\left(r, \vartheta ; E, \beta_{2} ; t\right)=W_{r}\left(r ; E, \beta_{2}\right)+W_{\vartheta}\left(\vartheta ; E, \beta_{2}\right)-E t
$$

for $W_{r} \in C^{2}((0, \infty)), W_{\vartheta} \in C^{2}([0,2 \pi))$. We make a further useful observation. Note that $\vartheta$ is a cyclic coordinate in (6.2.6). It was also discussed in Section 5.2 how cyclic coordinates can alter the form of $S$ to help the solving and so we follow (5.2.31) and rewrite (6.2.12) as

$$
S\left(r, \vartheta ; E, \beta_{2}, t\right)=W_{r}\left(r ; E, \beta_{2}\right)+\beta_{2} \vartheta-E t
$$

where $\beta_{2}$ is the conserved coordinate $p_{\vartheta}$ solving

$$
\beta_{2}=\frac{\partial W_{\vartheta}}{\partial \vartheta}, \quad W_{\vartheta}\left(\vartheta ; E, \beta_{2}\right):=\beta_{2} \vartheta
$$

The generating function derivatives at (6.2.9) and (6.2.10) as well as the HamiltonJacobi equation at (6.2.11) now appear as

$$
\begin{gathered}
p_{r}=\frac{\partial W_{r}}{\partial r}, \quad p_{\vartheta}=\frac{\partial W_{\vartheta}}{\partial \vartheta} \\
Q_{r}=\frac{\partial W_{r}}{\partial E}-t, \quad Q_{\vartheta}=\frac{\partial W_{r}}{\partial \beta_{2}}+\vartheta
\end{gathered}
$$

and

$$
\sqrt{m^{2}+\left(\frac{\partial W_{r}}{\partial r}\right)^{2}+\frac{\beta_{2}^{2}}{r^{2}}}-m+V(r)=E
$$

respectively. We can rearrange (6.2.17) to have

$$
\beta_{2}= \pm r \sqrt{(E+m-V(r))^{2}-m^{2}-\left(\frac{\partial W_{r}}{\partial r}\right)^{2}} \text {. }
$$

Defining $f\left(\frac{\partial W_{\vartheta}}{\partial \vartheta}\right)$ and $g\left(r, \frac{\partial W_{r}}{\partial r}, E\right)$ as the left and right-hand sides of (6.2.18) respectively we have the form of (5.2.17). Hence we can argue as was done there to deduce that

$$
f\left(\frac{\partial W_{\vartheta}}{\partial \vartheta}\right)=\beta_{2}
$$

and

$$
g\left(r, \frac{\partial W_{r}}{\partial r}, E\right)=\beta_{2}
$$


Therefore by Definition 5.2.4 the coordinate $\vartheta$ is separable and $\beta_{2}$ is a separation constant. Solving (6.2.19) for $\frac{\partial W_{r}}{\partial r}$ gives

$$
\frac{\partial W_{r}}{\partial r}= \pm \sqrt{(E+m-V(r))^{2}-m^{2}-\frac{\beta_{2}^{2}}{r^{2}}}
$$

implying that

$$
W_{r}\left(r, \vartheta ; E, \beta_{2}\right)= \pm \int \sqrt{(E+m-V(r))^{2}-m^{2}-\frac{\beta_{2}^{2}}{r^{2}}} \mathrm{~d} r
$$

Therefore using (6.2.13) Hamilton's principal function follows as

$$
S\left(r, \vartheta ; E, \beta_{2} ; t\right)= \pm \int \sqrt{(E+m-V(r))^{2}-m^{2}-\frac{\beta_{2}^{2}}{r^{2}}} \mathrm{~d} r+\beta_{2} \vartheta-E t .
$$

Using the derivatives from (6.2.15) we find $p_{r}$ to be

$$
p_{r}=\frac{\partial W_{r}}{\partial r}= \pm \sqrt{(E+m-V(r))^{2}-m^{2}-\frac{\beta_{2}^{2}}{r^{2}}}
$$

with $p_{\vartheta}$ to be already what we know:

$$
p_{\vartheta}=\frac{\partial W_{\vartheta}}{\partial \vartheta}=\beta_{2} .
$$

For the coordinates $r$ and $\vartheta$, we look to the derivatives from (6.2.16) to find that they satisfy

$$
\gamma_{1}=Q_{1}=\frac{\partial W_{r}}{\partial E}-t
$$

or

$$
\gamma_{1}+t= \pm \int \frac{r(E+m-V(r))}{\sqrt{r^{2}\left((E+m-V(r))^{2}-m^{2}\right)-\beta_{2}^{2}}} \mathrm{~d} r
$$

and

$$
\gamma_{2}=Q_{2}=\frac{\partial W_{r}}{\partial \beta_{2}}+\vartheta
$$

or

$$
\gamma_{2} \pm \int \frac{\beta_{2}}{r \sqrt{r^{2}\left((E+m-V(r))^{2}-m^{2}\right)-\beta_{2}^{2}}} \mathrm{~d} r=\vartheta
$$

Whenever the integration is possible, equation (6.2.24) can be used to find $r$ in terms of $t$. We can then use this with (6.2.25) to find $\vartheta$. As before introducing initial conditions eliminates the constants $\gamma_{i}, i=1,2$, leaving them in terms of the initial values and $E$. 
Looking back to the solution (4.3.29) we obtained from the corresponding twodimensional relativistic example in Section 4.3, we see that it coincides with (6.2.24) above. In fact the conjugate momenta (6.2.22) and (6.2.23) also agree with what was deduced from this example.

There are some conditions we need to impose such that everything is well-defined:

(i) $E+m-V(r) \neq 0$,

(ii) $(E+m-V(r))^{2}-m^{2}-\frac{\beta_{2}^{2}}{r^{2}}>0$.

Following the method seen at Example 4.1.2 we deduce that $p_{r} \neq 0$ is sufficient for the above to hold.

Example 6.2.3. The Hamilton function whose dynamics we want to study is $\mathcal{H} \in$ $C^{1}\left(\mathbb{R}^{6}\right)$ such that

$$
\mathcal{H}(q, p)=\sqrt{m^{2}+\|p\|^{2}}-m+V(q) .
$$

We employ spherical polar coordinates to obtain an equivalent form to (6.2.26) given by $\mathcal{H}_{3, R} \in C^{1}\left((0, \infty) \times[0, \pi] \times[0,2 \pi) \times \mathbb{R} \backslash\{0\} \times \mathbb{R}^{2}\right)$ such that

$$
\mathcal{H}_{3, R}\left(r, \phi, \theta, p_{r}, p_{\phi}, p_{\theta}\right)=\sqrt{m^{2}+p_{r}^{2}+\frac{p_{\phi}^{2}}{r^{2}}+\frac{p_{\theta}^{2}}{r^{2} \sin ^{2} \phi}}-m+V(r) .
$$

Using the Hamilton-Jacobi theory we solve the Hamilton-Jacobi equation to obatin the generating function that induces a canonical transformation such that the transformed Hamilton function is zero and the transformed coordinates are constant:

$$
Q_{r}=\gamma_{1}, \quad Q_{\phi}=\gamma_{2}, \quad Q_{\theta}=\gamma_{3}, \quad P_{r}=E, \quad P_{\phi}=\beta_{2}, \quad P_{\theta}=\beta_{3},
$$

where $E$ is the conserved value of (6.2.27) determined by the initial conditions. Using the above conserved coordinates at (6.2.28) with the generating function derivatives the solutions to the associated Hamilton's equations of (6.2.27) are found to satisfy

$$
\begin{aligned}
& p_{r}= \pm \sqrt{(E+m-V(r))^{2}-m^{2}-\frac{\beta_{2}^{2}}{r^{2}}}, \\
& p_{\phi}= \pm \sqrt{\beta_{2}^{2}-\frac{\beta_{3}^{2}}{\sin ^{2} \phi}}, \\
& p_{\theta}=\beta_{3} \text {, } \\
& \gamma_{1}+t= \pm \int \frac{r(E+m-V(r))}{\sqrt{r^{2}\left((E+m-V(r))^{2}-m^{2}\right)-\beta_{2}^{2}}} \mathrm{~d} r
\end{aligned}
$$




$$
\gamma_{2} \pm \int \frac{\beta_{2}}{r \sqrt{r^{2}\left((E+m-V(r))^{2}-m^{2}\right)-\beta_{2}^{2}}} \mathrm{~d} r= \pm \int \frac{\beta_{2} \sin \phi}{\sqrt{\beta_{2}^{2} \sin ^{2} \phi-\beta_{3}^{2}}} \mathrm{~d} \phi
$$

and

$$
\gamma_{3} \pm \int \frac{\beta_{3}}{\sin \phi \sqrt{\beta_{2}^{2} \sin ^{2} \phi-\beta_{3}^{2}}} \mathrm{~d} \phi=\theta
$$

where $\gamma_{i}, i=1,2,3$, are constants of integration that may be determined using the initial conditions.

Recollecting the solution (4.3.47) from Example 4.3.3, the corresponding example to this one covered in Section 4.3, we see that (6.2.32) agrees with what is seen there. In fact the conjugate momenta (6.2.29), (6.2.30) and (6.2.31) also agree with the contents of Example 4.3.3. The solutions may be solved completely as at Example 6.1.3.

There are some conditions we need to impose such that everything is well-defined:

(i) $E+m-V(r) \neq 0$,

(ii) $r^{2}\left((E+m-V(r))^{2}-m^{2}\right)-\beta_{2}^{2}>0$,

(iii) $\beta_{2}^{2}-\frac{\beta_{3}^{2}}{\sin ^{2} \phi}>0$.

As with Example 4.3.3, we follow the method at Example 4.1.3 to deduce that $p_{r} \neq 0$ is sufficient for the above to hold.

\subsection{Relativistic Dynamics with a Fractional Power}

We recall from the introduction of Section 4.3 that a problem with the Hamilton function

$$
\mathcal{H}_{R}(q, p)=\sqrt{m^{2}+\|p\|^{2}}-m+V(q), \quad m>0,
$$

was the lack of coercivity of its kinetic part. However, we present here an alternate take on the example where we now take the kinetic part of the Hamilton function to be

$$
\psi(p)=\left(m^{2}+\|p\|^{2}\right)^{\alpha}-m^{2 \alpha}, \quad \frac{1}{2}<\alpha<1,
$$

which is coercive. Note (6.3.2) is continuous negative definite by Proposition 2.3 from Chapter 2. It is also convex for $n=1,2$ and 3. We add $-m^{2 \alpha}$ in (6.3.2) so that $d_{\psi}:=\psi^{\frac{1}{2}}$ maintains the characteristic properties of a metric (recall Chapter $2)$. As we will see through the examples below we are able to use the theory of Hamilton-Jacobi to study the associated dynamics of (6.3.2) as we did with (6.3.1). 
One could read the example associated with (6.3.2) as a generalisation of the examples considered so far for the relativstic dynamics. Ignoring coercivity the example at (6.3.1) acts as a special case of (6.3.2) where $\alpha=\frac{1}{2}$. This is similar to a generalisation done with the Harmonic Oscillator by introducing a fractional power: recall our Hamilton function

$$
\mathcal{H}(q, p)=\frac{1}{\alpha}\|p\|^{\alpha}+\frac{1}{\alpha^{*}}\|q\|^{\alpha^{*}}, \quad 1<\alpha \leq 2, \quad \alpha^{*}=\frac{\alpha}{\alpha-1},
$$

acted as a generalisation of

$$
\mathcal{H}(q, p)=\frac{1}{2}\|p\|^{2}+\frac{1}{2}\|q\|^{2}
$$

from classical mechanics.

To follow previous examples related to relativistic dynamics we take the potential to be the conjugate of the kinetic term. To find the conjugate of (6.3.2) we notice that it is rotational invariant. Hence we may use Theorem 1.16 from Chapter 1 to find this. Through this we require

$$
\zeta_{\psi, \alpha}(s):=4 \alpha^{2}\left(m^{2}+s\right)^{2(\alpha-1)} s
$$

to be bijective for $s \geq 0$. The above function is strictly increasing for $s \geq 0$ hence we know that it is bijective and that an inverse exists. However it is difficult to explicitly calculate the inverse. Therefore we turn to finding a particular case where we can find an inverse. Taking $\alpha=\frac{3}{4}$ and making the substitution $s=r^{-1}$, we are able to find the inverse of

$$
\zeta_{\psi, \frac{3}{4}}(s)=\frac{9}{4}\left(m^{2}+s\right)^{-\frac{1}{2}} s
$$

to be

$$
\zeta_{\psi, \frac{3}{4}}^{-1}(s):=\frac{4 m^{2} s}{\sqrt{81 m^{2}+4 s^{2}}-2 s} .
$$

Through this, we use Theorem 1.16 to calculate the conjuagate function $\psi^{*}$ to be

$$
\begin{aligned}
\psi^{*}(\eta)=m^{\frac{3}{2}}(1- & \left(\frac{2\|\eta\|^{2}+\sqrt{81 m^{2}+4\|\eta\|^{4}}}{-2\|\eta\|^{2}+\sqrt{81 m^{2}+4\|\eta\|^{4}}}\right)^{\frac{3}{4}} \\
+ & \left.\frac{6\|\eta\|^{2}\left(\frac{2\|\eta\|^{2}+\sqrt{81 m^{2}+4\|\eta\|^{4}}}{-2\|\eta\|^{2}+\sqrt{81 m^{2}+4\|\eta\|^{4}}}\right)^{\frac{3}{4}}}{2\|\eta\|^{2}+\sqrt{81 m^{2}+4\|\eta\|^{4}}}\right) .
\end{aligned}
$$

Note the $\sqrt{81 m^{2}+4\|\eta\|^{4}}$ is always well-defined given $m \neq 0$. Further we have no singularity at (6.3.3) since $\sqrt{81 m^{2}+4\|\eta\|^{4}}>2\|\eta\|^{2}$. 
Below we present the one, two and three-dimensional cases of investigating the associated dynamics of $\mathcal{H}(q, p)=\psi(p)+V(q)$ where $\psi$ is as at (6.3.2). Thanks to the theory of Hamilton-Jacobi we are able to do this independently of a specific potential in most cases. However should we require a specific potential we can take $V(q)=\psi^{*}(q)$ now that we have (6.3.3). The examples also follow a similar method to the corresponding one, two and three-dimensional examples in the previous section. Hence we are more brief here in presenting the results, knowing that the full calculations may be illustrated by analogously following the examples before.

To follow the first of the next collection of examples are graphics similar to what was seen in Chapter 4. They are constructed using Mathematica and serve the same purpose as before in giving a better idea of how the solutions are behaving. However, due to the need of an explicit potential to form these graphics, we pick up the potential from (6.3.3).

Example 6.3.1. We would like to study the dynamics associated with the function $\mathcal{H}_{1, R, \alpha} \in C^{1}(\mathbb{R} \times \mathbb{R} \backslash\{0\})$ such that

$$
\mathcal{H}_{1, R, \alpha}(q, p)=\left(m^{2}+p^{2}\right)^{\alpha}-m^{2 \alpha}+V(q) .
$$

We take the same approach as in Example 6.2.1 and use the Hamilton-Jacobi theory to solve the Hamilton-Jacobi equation to find the generating function. This function induces a canonical transformation so that the transformed Hamilton function is zero and the transformed coordinates are constant:

$$
Q=\gamma, \quad P=E,
$$

where $E$ is the conserved value of the Hamilton function (6.3.4) determined by the initial conditions. Using the theory of Hamilton-Jacobi the coordinates are found to be

$$
p= \pm \sqrt{\left(E+m^{2 \alpha}-V(q)\right)^{\frac{1}{\alpha}}-m^{2}}
$$

and

$$
\gamma+t= \pm \frac{1}{2 \alpha} \int \frac{\left(E+m^{2 \alpha}-V(q)\right)^{\frac{1-\alpha}{\alpha}}}{\sqrt{\left(E+m^{2 \alpha}-V(q)\right)^{\frac{1}{\alpha}}-m^{2}}} \mathrm{~d} q,
$$

where $\gamma$ is a constant of integration which may be determined using the initial conditions. We can now completely solve the coordinates analogously to how it is done in Example 6.1.1.

As with some previous examples, there are conditions we must check so that the above is well-defined:

(i) $E+m^{2 \alpha}-V(q)>0$, 
(ii) $\left(E+m^{2 \alpha}-V(q)\right)^{\frac{1}{\alpha}}-m^{2}>0$.

Following a similar method to what was seen at Example 4.1.2, we can substitute (6.3.1) as the value of $E$ into the above conditions to deduce that $p \neq 0$ is sufficient for them to hold.

Note taking $\alpha=\frac{1}{2}$ in this example obtains the results of Example 6.2.1, as expected.

We now consider some corresponding graphics. Taking the potential to be the conjugate of the kinetic term when $\alpha=\frac{3}{4}$, we have the following special case of the Hamilton function:

$$
\begin{gathered}
\mathcal{H}_{1, R, \frac{3}{4}}(q, p)=\left(m^{2}+p^{2}\right)^{\frac{3}{4}}-m^{\frac{3}{2}}\left(\left(\frac{2 q^{2}+\sqrt{81 m^{2}+4 q^{4}}}{-2 q^{2}+\sqrt{81 m^{2}+4 q^{4}}}\right)^{\frac{3}{4}}\right. \\
\left.+\frac{6 q^{2}\left(\frac{2 q^{2}+\sqrt{81 m^{2}+4 q^{4}}}{-2 q^{2}+\sqrt{81 m^{2}+4 q^{4}}}\right)^{\frac{3}{4}}}{2 q^{2}+\sqrt{81 m^{2}+4 q^{4}}}\right)
\end{gathered}
$$

with Hamilton's equations:

$$
\left\{\begin{array}{l}
\dot{q}=\frac{3 p}{2\left(m^{2}+p^{2}\right)^{\frac{1}{4}}}, \\
\dot{p}=-\frac{18 m^{\frac{3}{2}} q}{\left(-2 q^{2}+\sqrt{81 m^{2}+4 q^{4}}\right)\left(81+\frac{4 q^{2}\left(2 q^{2}+\sqrt{81 m^{2}+4 q^{4}}\right)}{m^{2}}\right)^{\frac{1}{4}}} .
\end{array}\right.
$$

For selected values of $m$, below are some examples of the corresponding phase diagrams constructed using Mathematica. The horizontal axis represents the coordinate $q$ where the vertical corresponds to $p$. 


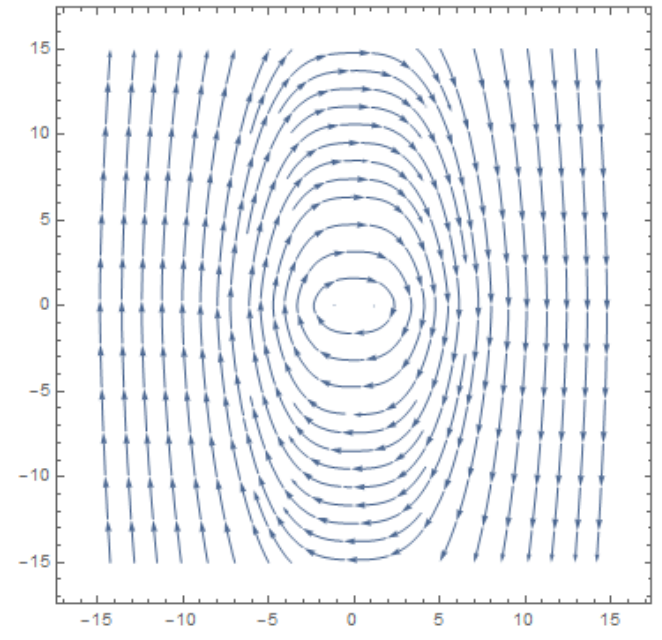

Figure 6.3.1: $m=0.1$

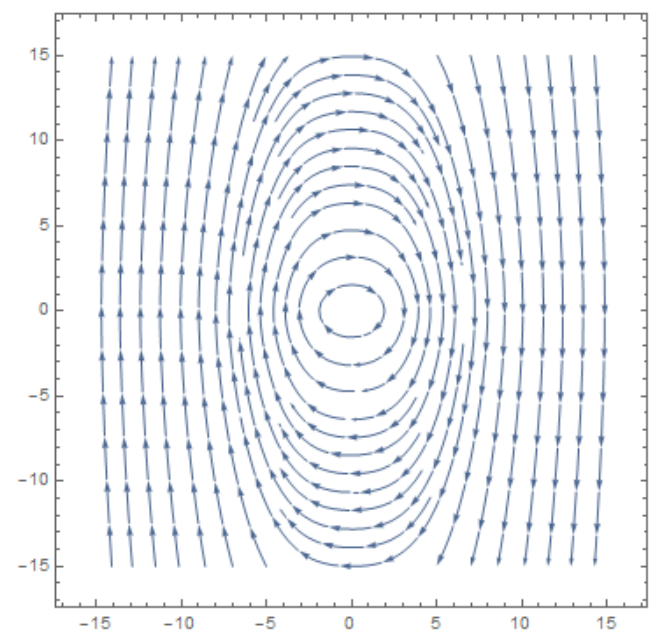

Figure 6.3.3: $m=1$

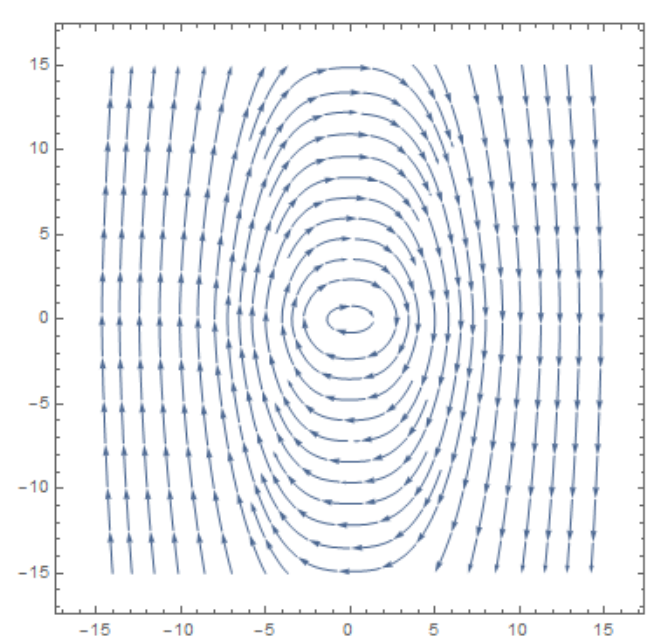

Figure 6.3.2: $m=0.5$

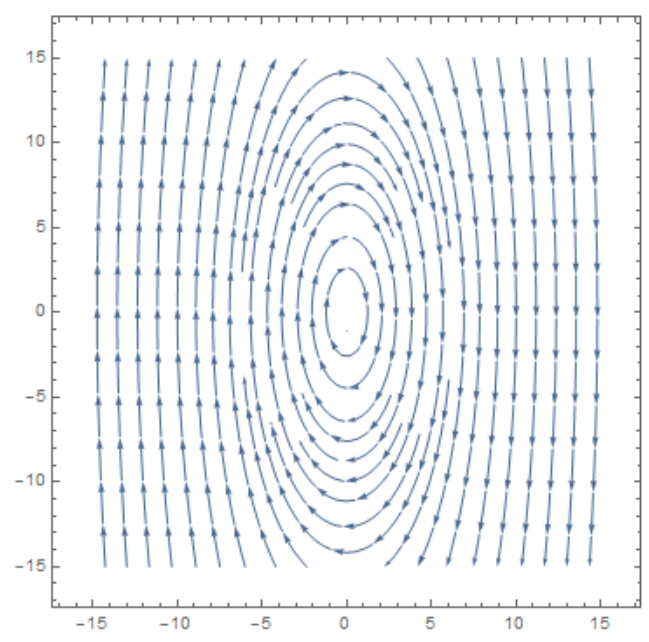

Figure 6.3.4: $m=10$

Observing the above figures we can see that the solutions have a symmetric property when reflected over the lines $q=-p, p=0$ and $q=0$. In other words given the solutions for when $q \geq 0$ and $p \geq 0$, we can solve the cases for $q<0$ and $p<0$; $q \geq 0$ and $p<0 ; q<0$ and $p \geq 0$ by reflecting over $q=-p, p=0$ and $q=0$ respectively in the phase space and taking the appropiate sign. Additionally, observing the figures above we see that they suggest a periodic property in the solutions, in particular demonstrating behaviour quite close to what we expect from the Harmonic Oscillator in classical mechanics as $m$ gets smaller. The figures compare well with those in Example 4.3.1 where $\alpha=\frac{1}{2}$, with the path becoming taller and thinner as 
$m$ gets larger.

Example 6.3.2. The Hamilton function whose dynamics we want to study is $\mathcal{H} \in$ $C^{1}\left(\mathbb{R}^{4}\right)$ such that

$$
\mathcal{H}(q, p)=\left(m^{2}+\|p\|^{2}\right)^{\alpha}-m^{2 \alpha}+V(q) .
$$

We employ polar coordinates to obtain an equivalent form to (6.3.7) given by $\mathcal{H}_{2, R, \alpha} \in$ $C^{1}((0, \infty) \times[0,2 \pi) \times \mathbb{R} \backslash\{0\} \times \mathbb{R})$ such that

$$
\mathcal{H}_{2, R, \alpha}\left(r, \vartheta, p_{r}, p_{\vartheta}\right)=\left(m^{2}+p_{r}^{2}+\frac{p_{\vartheta}^{2}}{r^{2}}\right)^{\alpha}-m^{2 \alpha}+V(r) .
$$

We solve the corresponding Hamilton-Jacobi equation to find the generating function. This function induces a canonical transformation such that the transformed Hamilton function is zero and the transformed coordinates are constant:

$$
Q_{r}=\gamma_{1}, \quad Q_{\vartheta}=\gamma_{2}, \quad P_{r}=E, \quad P_{\vartheta}=\beta_{2},
$$

where $E$ is the conserved value of (6.3.8) determined by the initial conditions. Using the above conserved coordinates with the generating function derivatives the solutions are found to satisfy

$$
\begin{gathered}
p_{r}= \pm \sqrt{\left(E+m^{2 \alpha}-V(r)\right)^{\frac{1}{\alpha}}-m^{2}-\frac{\beta_{2}^{2}}{r^{2}}} \\
p_{\vartheta}=\beta_{2} \\
\gamma_{1}+t= \pm \frac{1}{2 \alpha} \int \frac{r\left(E+m^{2 \alpha}-V(r)\right)^{\frac{1-\alpha}{\alpha}}}{\sqrt{r^{2}\left(\left(E+m^{2 \alpha}-V(r)\right)^{\frac{1}{\alpha}}-m^{2}\right)-\beta_{2}^{2}}} \mathrm{~d} r
\end{gathered}
$$

and

$$
\gamma_{2} \pm \int \frac{\beta_{2}}{r \sqrt{r^{2}\left(\left(E+m^{2 \alpha}-V(r)\right)^{\frac{1}{\alpha}}-m^{2}\right)-\beta_{2}^{2}}} \mathrm{~d} r=\vartheta
$$

where $\gamma_{i}, i=1,2$, are constants of integration that may be determined using the initial conditions. We can now completely solve the coordinates analogously how it is done in Example 6.2.2.

There are some conditions we need to impose such that the above well-defined:

(i) $E+m^{2 \alpha}-V(r)>0$, 
(ii) $\left(E+m^{2 \alpha}-V(r)\right)^{\frac{1}{\alpha}}-m^{2}-\frac{\beta_{2}^{2}}{r^{2}}>0$.

We deal with these as we did in Example 6.3.1. Following the method seen there we deduce that $p_{r} \neq 0$ is sufficient for the above to hold.

Example 6.3.3. The Hamilton function whose dynamics we want to study is $\mathcal{H} \in$ $C^{1}\left(\mathbb{R}^{6}\right)$ such that

$$
\mathcal{H}(q, p)=\left(m^{2}+\|p\|^{2}\right)^{\alpha}-m^{2 \alpha}+V(q) .
$$

We employ spherical polar coordinates to obtain an equivalent form to (6.3.9) given by $\mathcal{H}_{3, R, \alpha} \in C^{1}\left((0, \infty) \times[0, \pi] \times[0,2 \pi) \times \mathbb{R} \backslash\{0\} \times \mathbb{R}^{2}\right)$ such that

$$
\mathcal{H}_{3, R, \alpha}\left(r, \phi, \theta, p_{r}, p_{\phi}, p_{\theta}\right)=\left(m^{2}+p_{r}^{2}+\frac{p_{\phi}^{2}}{r^{2}}+\frac{p_{\theta}^{2}}{r^{2} \sin ^{2} \phi}\right)^{\alpha}-m^{2 \alpha}+V(r) .
$$

We solve the corresponding Hamilton-Jacobi equation to find the generating function. This function induces a canonical transformation such that the transformed Hamilton function is zero and the transformed coordinates are constant:

$$
Q_{r}=\gamma_{1}, \quad Q_{\phi}=\gamma_{2}, \quad Q_{\theta}=\gamma_{3}, \quad P_{r}=E, \quad P_{\phi}=\beta_{2}, \quad P_{\theta}=\beta_{3},
$$

where $E$ is the conserved value of (6.3.10) determined by the initial conditions. Using the above conserved coordinates with the generating function derivatives the solutions are found to satisfy

$$
\begin{aligned}
& p_{r}= \pm \sqrt{\left(E+m^{2 \alpha}-V(r)\right)^{\frac{1}{\alpha}}-m^{2}-\frac{\beta_{2}^{2}}{r^{2}}} \\
& p_{\phi}= \pm \sqrt{\beta_{2}^{2}-\frac{\beta_{3}^{2}}{\sin ^{2} \phi}} \\
& p_{\theta}=\beta_{3}, \\
& \gamma_{1}+t= \pm \frac{1}{2 \alpha} \int \frac{r\left(E+m^{2 \alpha}-V(r)\right)^{\frac{1-\alpha}{\alpha}}}{\sqrt{r^{2}\left(\left(E+m^{2 \alpha}-V(r)\right)^{\frac{1}{\alpha}}-m^{2}\right)-\beta_{2}^{2}}} \mathrm{~d} r \\
& \gamma_{2} \pm \int \frac{\beta_{2}}{r \sqrt{r^{2}\left(\left(E+m^{2 \alpha}-V(r)\right)^{\frac{1}{\alpha}}-m^{2}\right)-\beta_{2}^{2}}} \mathrm{~d} r= \pm \int \frac{\beta_{2} \sin \phi}{\sqrt{\beta_{2}^{2} \sin ^{2} \phi-\beta_{3}^{2}}} \mathrm{~d} \phi
\end{aligned}
$$

and

$$
\gamma_{3} \pm \int \frac{\beta_{3}}{\sin \phi \sqrt{\beta_{2}^{2} \sin ^{2} \phi-\beta_{3}^{2}}} \mathrm{~d} \phi=\theta
$$


where $\gamma_{i}, i=1,2,3$, are constants of integration that may be determined using the initial conditions. We can now completely solve the coordinates analogously how it is done in Example 6.2.3.

There are some conditions we need to impose such that the above are well-defined:

(i) $E+m^{2 \alpha}-V(r)>0$,

(ii) $\left(E+m^{2 \alpha}-V(r)\right)^{\frac{1}{\alpha}}-m^{2}-\frac{\beta_{2}^{2}}{r^{2}}>0$,

(iii) $\beta_{2}^{2}-\frac{\beta_{3}^{2}}{\sin ^{2} \phi}>0$.

We deal with these as we did in Example 4.1.3. Following the method seen there we deduce that $p_{r} \neq 0$ is sufficient for the above to hold. 


\section{Appendix A}

\section{Hypergeometric Series}

The statements and results contained in this appendix are in reference to [25], [1] and [3]. Before defining the hypergeometric series we need shifted factorials:

Definition A.1. For any $a \in \mathbb{R}$ or $\mathbb{C}$ the shifted factorial or Pochhammer symbol is defined by

$$
(a)_{n}=a(a+1) \cdot \ldots \cdot(a+n-1), \quad n>0,(a)_{0}=1 .
$$

Definition A.2. The hypergeometric series is defined as

$$
{ }_{r} F_{s}\left(a_{1}, \ldots, a_{r} ; b_{1}, \ldots, b_{s} ; z\right)=\sum_{n=0}^{\infty} \frac{\left(a_{1}\right)_{n} \cdot \ldots \cdot\left(a_{r}\right)_{n}}{n !\left(b_{1}\right)_{n} \cdot \ldots \cdot\left(b_{s}\right)_{n}} z^{n}
$$

with $r$ complex numerator parameters $a_{1}, \ldots, a_{r}, s$ complex denominator parameters $b_{1}, \ldots, b_{s}$, and $z$ a complex variable. For (A.1) to be well-defined, each $b_{k}, k=$ $1, \ldots, s$, cannot be a negative integer or zero.

Hypergeometric series can be used to represent many well-known functions by taking specific values for the parameters $a_{1}, \ldots, a_{r}$ and $b_{1}, \ldots, b_{s}$. An example is

$$
\sin ^{-1} z=z_{2} F_{1}\left(\frac{1}{2}, \frac{1}{2} ; \frac{3}{2} ; z^{2}\right)
$$

The convergence of (A.1) is discussed in the next theorem:

Theorem A.3. Suppose we have the hypergeometric series ${ }_{r} F_{s}$ as in Definition A.2.

(i) If $r \leq s$ the series converges absolutely for all $z$,

(ii) if $r=s+1$ the series converges absolutely for $|z|<1$, 
(iii) if $r>s+1$ then the series diverges for all $z \neq 0$.

Proof. The proof is found at [1, page 62].

There are further convergence properties when $|z|=1$ and $r=s+1$ :

Theorem A.4. Consider ${ }_{s+1} F_{s}\left(a_{1}, \ldots, a_{s+1} ; b_{1}, \ldots, b_{s} ; z\right)$ such that $|z|=1$. Then

(i) if $\operatorname{Re}\left(\sum_{k=1}^{s} b_{k}-\sum_{l=1}^{s+1} a_{l}\right)>0$, the series converges absolutely,

(ii) if $z=e^{i \theta} \neq 1$ and $0 \geq \operatorname{Re}\left(\sum_{k=1}^{s} b_{k}-\sum_{l=1}^{s+1} a_{l}\right)>-1$, the series converges conditionally,

(iii) if $\operatorname{Re}\left(\sum_{k=1}^{s} b_{k}-\sum_{l=1}^{s+1} a_{l}\right) \leq-1$, the series diverges.

Proof. The proof can be found at [1, pages 62 to 63$]$.

Of most use to us is when $r=2$ and $s=1$ in (A.1) of Definition A.2.

Definition A.5. The hypergeometric series ${ }_{2} F_{1}(a, b ; c ; z)$ has the form

$$
{ }_{2} F_{1}(a, b ; c ; z):=\sum_{n=0}^{\infty} \frac{(a)_{n}(b)_{n}}{n !(c)_{n}} z^{n}
$$

where $a, b, c$ and $z$ are all complex and $(.)_{n}$ is the shifted factorial from Definition A.1. The series is defined for $|z|<1$. This particular form is known as Gauss' series, the (ordinary) hypergeometric series or the Gauss hypergeometric series.

Relating the above to its generalisation at Definition A.2, note $a$ and $b$ correspond to $a_{1}, \ldots, a_{r}$, and $c$ corresponds to $b_{1}, \ldots, b_{s}$.

Remark A.6. As a function ${ }_{2} F_{1}$ does have a continuation to the complex plane. However this is not necessary for what we want to do in this document and so we refer to [1, Chapter 2] for more details.

We see in Chapter 4 how hypergeometric series appear in solutions of differential equations. Hence of interest is this observation from [3]: 
Theorem A.7. The hypergeometric series (A.1) is the solution of the generalised hypergeometric equation:

$$
L_{(a),(b)} u(x)=0, \quad u(0)=1,
$$

where by $L_{(a),(b)}$ we denote the differential operator

$$
L_{(a),(b)}=z^{-1} D \prod_{k=1}^{s}\left(D+b_{k}-1\right)-\prod_{l=1}^{r}\left(D+a_{l}\right)
$$

for $D=z \frac{\mathrm{d}}{\mathrm{d} z}$.

Proof. This is seen at [3, page 265].

Taking $s=1$ and $r=2$ in Theorem A.7 we obtain a special case of the above as seen at $[3]$ :

Corollary A.8. Let $_{2} F_{1}(a, b ; c ; z)$ be a hypergeometric series as in Definition A.5. Then it is a solution to the second-order differential equation

$$
L_{a b c} u(z)=z(1-z) \frac{\mathrm{d}^{2} u}{\mathrm{~d} z^{2}}+[c-(a+b+1) z] \frac{\mathrm{d} u}{\mathrm{~d} z}-a b u=0
$$

for some function $u \in C^{2}(\mathbb{C})$. The equation $(A .5)$ is known as the hypergeometric equation (see [68, page 283]) or Euler's hypergeometric differential equation as in [1, page 75]. The operator $L_{a b c}$ is the hypergeometric operator.

Remark A.9. Similar to what was said in Remark A.6, solutions for Euler's hypergeometric differential equation may also be extended. However this will also not be of use to us in this particular document and we refer to [3] for further details.

An example of solving the above differential equation can be found below.

Example A.10. (Following [68, page 283])

The differential equation

$$
z\left(z \frac{\mathrm{d}}{\mathrm{d} z}+a\right)\left(z \frac{\mathrm{d}}{\mathrm{d} z}+b\right) u-\left(z \frac{\mathrm{d}}{\mathrm{d} z}-\alpha\right)\left(z \frac{\mathrm{d}}{\mathrm{d} z}-\beta\right) u=0
$$

has the solution

$$
z^{\alpha}{ }_{2} F_{1}(a+\alpha, b+\alpha ; \alpha-\beta+1 ; z) .
$$

A further solution to (A.6) also comes from [23, page 209]:

$$
z^{1-c} F_{1}(a+1-c, b+1-c ; 2-c ; z) .
$$




\section{Appendix B}

\section{Full Calculations of Examples}

Here we provide the full details for the calculations behind the examples for the Harmonic Oscillator and the Kepler problem in Chapter 4. The method used to solve the two-dimensional examples for either case is almost identical. The only clear distinction is the unique potential $V$ for each case. Hence below we present only one example for each of two-dimensional cases that include a general potential $V$. As before the one-dimensional cases have been presented as separate examples.

One may also notice the lack of reference to the examples corresponding to the relativistic dynamics. This is largely due to the same reasoning again as each of its examples follow the same method as is found with the Harmonic Oscillator or Kepler problem. Hence to determine the solutions found at Section 4.3 for either one or two dimensions, we only need to follow the procedure shown in the corresponding example below and use what is shown at Section 4.3 as a guide for what we are aiming for. In the case of one-dimension, this should be even easier since the abscence of absolute values in the problems means we need not consider cases of different signs as is required in Examples B.1 and B.2.

A final point is that conditions should be taken to guarantee that the solutions are well-defined. We recall that such conditions were taken in the corresponding examples to the ones below at Chapter 4. Hence we do not repeat them here and only concentrate on demonstrating the calculations themselves.

We begin with the one-dimensional example for the Harmonic Oscillator:

Example B.1. We would like to study the dynamics associated with the Hamilton function $\mathcal{H} \in C^{1}\left(\mathbb{R}^{2}\right)$

$$
\mathcal{H}(q, p)=\frac{1}{\alpha}|p|^{\alpha}+\frac{1}{\alpha^{*}}|q|^{\alpha^{*}}
$$

for $1<\alpha \leq 2$ and $\alpha^{*}=\frac{\alpha}{\alpha-1}$. Hamilton's equations follow as

$$
\left\{\begin{array}{l}
\dot{q}=p|p|^{\alpha-2}, \\
\dot{p}=-q|q|^{\alpha^{*}-2}
\end{array}\right.
$$


Due to the absolute values the system of differential equations at (B.2) is not so easy to solve. We will consider the system for four different cases, namely when $q \geq 0$ and $p \geq 0, q \geq 0$ and $p<0, q<0$ and $p \geq 0$ and $q<0$ and $p<0$. To follow will be a method solving (B.2) for $q \geq 0$ and $p \geq 0$. The method may then be carried out analogously for the three remaining cases, hence giving a symmetrical property of sorts. However we do not do the latter. Instead we conclude by providing a presentation of the solutions that covers each possible case for the sign of $q$ and $p$.

Let us begin with $q \geq 0$ and $p \geq 0$, i.e. the Hamilton function (B.1) appears as

$$
\mathcal{H}(q, p)=\frac{1}{\alpha} p^{\alpha}+\frac{1}{\alpha^{*}} q^{\alpha^{*}}
$$

We solve

$$
\left\{\begin{array}{l}
\dot{q}=p^{\alpha-1}, \\
\dot{p}=-q^{\alpha^{*}-1}, \quad q, p \geq 0 .
\end{array}\right.
$$

We set initial conditions to employ, namely $q\left(t_{0}\right)=q_{0}$ and $p\left(t_{0}\right)=p_{0}$. Once the general solutions have been found, these conditions will be included to obtain the complete solutions for the initial value problem.

We know from Section 3.3 that introducing conserved quantites can simplify the solving. From Theorem 3.3.1 the Hamilton function has a constant value E determined by the initial conditons, i.e.

$$
\mathcal{H}(q(t), p(t))=\mathcal{H}\left(q_{0}, p_{0}\right)=E
$$

Rearranging (B.5) we can obtain further equations for $q$ and $p$ :

$$
q=\alpha^{* \frac{1}{\alpha^{*}}}\left(E-\frac{1}{\alpha} p^{\alpha}\right)^{\frac{1}{\alpha^{*}}}>0 \text { and } p=\alpha^{\frac{1}{\alpha}}\left(E-\frac{1}{\alpha^{*}} q^{\alpha^{*}}\right)^{\frac{1}{\alpha}}>0 .
$$

Substituting these expressions into (B.4) we obtain a new system of decoupled ODE's:

$$
\left\{\begin{array}{l}
\dot{q}=\alpha^{\frac{1}{\alpha^{*}}}\left(E-\frac{1}{\alpha^{*}} q^{\alpha^{*}}\right)^{\frac{1}{\alpha^{*}}} \\
\dot{p}=-\alpha^{* \frac{1}{\alpha}}\left(E-\frac{1}{\alpha} p^{\alpha}\right)^{\frac{1}{\alpha}}
\end{array}\right.
$$

Using the method of separation of variables with the first equation of (B.7) we have

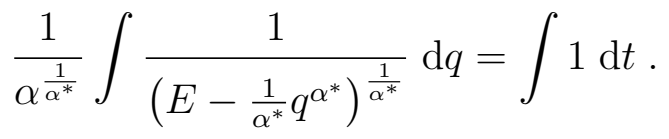

Integrating gives

$$
(\alpha E)^{-\frac{1}{\alpha^{*}}} q_{2} F_{1}\left(\frac{\alpha-1}{\alpha}, \frac{\alpha-1}{\alpha} ; \frac{2 \alpha-1}{\alpha} ; \frac{q^{\alpha^{*}}}{\alpha^{*} E}\right)=t+A_{1}
$$


where $A_{1}$ denotes the constant obtained through integration. It is worth noting that the term $q^{\alpha^{*}}$ is well-defined since we have taken the assumption that $q \geq 0$.

By Definition A.5 the hypergeometric series contained in (B.9) converges for

$$
\left|\frac{q^{\alpha^{*}}}{\alpha^{*} E}\right|<1
$$

or

$$
|q|<\left(\alpha^{*} E\right)^{\frac{1}{\alpha^{*}}} .
$$

In fact the inequality (B.10) agrees with what is needed for the first equation of (B. 7 ) to be well-defined.

Using (B.9) the general solution for $q$ follows as

$$
q(t)=u_{1}^{-1}\left((\alpha E)^{\frac{1}{\alpha^{*}}}\left(t+A_{1}\right)\right)
$$

where the function $u_{1}$ is defined as

$$
u_{1}(q):=q_{2} F_{1}\left(\frac{\alpha-1}{\alpha}, \frac{\alpha-1}{\alpha} ; \frac{2 \alpha-1}{\alpha} ; \frac{q^{\alpha^{*}}}{\alpha^{*} E}\right) .
$$

More needs to be said about $u_{1}$, in particular about its domain and range. How does this connect to the existence of the inverse $u_{1}^{-1}$ ? To do this let us suppose that $u_{1}$ is defined and continuously differentiable on a neighbourhood of $x_{0}$. Then using the local inverse function theorem (see [64, page 175]), if $u_{1}^{\prime}\left(x_{0}\right) \neq 0$ then there exists a neighbourhood of $x_{0}$, let this be $M_{1}$, such that $\left.u_{1}\right|_{M_{1}}$ has a continuously differentiable inverse $u_{1}^{-1}$ defined on the image $M_{2}=u_{1}\left(M_{1}\right)$.

Using the fundamental theorem of calculus (see [46, page 176]) it holds that

$$
\int\left(1-\frac{x^{\alpha^{*}}}{\alpha^{*} E}\right)^{\frac{-1}{\alpha^{*}}} \mathrm{~d} q=u_{1}(x) .
$$

Therefore $u_{1}$ is the primitive of the mapping

$$
x \mapsto\left(1-\frac{x^{\alpha^{*}}}{\alpha^{*} E}\right)^{\frac{-1}{\alpha^{*}}} .
$$

Through this we see that $u_{1}^{\prime}\left(x_{0}\right) \neq 0$ is equivalent to

$$
\left|x_{0}\right|<\left(\alpha^{*} E\right)^{\frac{1}{\alpha^{*}}} .
$$

Thus, under the condition (B.14) the inverse $u_{1}^{-1}$ exists for every point in the domain of $u_{1}$. This gives $u_{1}$ as a mapping from $M_{1}$ to $M_{2}$. Notice the condition (B.14) 
coincides with the inequality we needed at (B.10). Hence (B.10) acts as the uniform condition for equation (B.9) and the inverse $u_{1}^{-1}$.

We now move on to the second equation in the system (B.7). Using the method of separation of variables we have

$$
-\frac{1}{\alpha^{* \frac{1}{\alpha}}} \int \frac{1}{\left(E-\frac{1}{\alpha} p^{\alpha}\right)^{\frac{1}{\alpha}}} \mathrm{d} p=\int 1 \mathrm{~d} t .
$$

Integrating then gives

$$
-\left(\alpha^{*} E\right)^{-\frac{1}{\alpha}} p_{2} F_{1}\left(\frac{1}{\alpha}, \frac{1}{\alpha} ; \frac{\alpha+1}{\alpha} ; \frac{p^{\alpha}}{\alpha E}\right)=t+A_{2},
$$

$A_{2}$ a further constant of integration. Note the term $p^{\alpha}$ is always well-defined since we have taken the assumption that $p \geq 0$. Again by Definition A.5 the hypergeometric series contained in (B.15) converges for

$$
|p|<(\alpha E)^{\frac{1}{\alpha}} .
$$

Similar to what we had with (B.10), the inequality (B.16) agrees with what is needed for the second equation of (B.7) to be well-defined.

Defining a further continuously differentiable function $u_{2}$ :

$$
u_{2}(p):=p_{2} F_{1}\left(\frac{1}{\alpha}, \frac{1}{\alpha} ; \frac{\alpha+1}{\alpha} ; \frac{p^{\alpha}}{\alpha E}\right),
$$

it holds that

$$
p(t)=u_{2}^{-1}\left(-\left(\alpha^{*} E\right)^{\frac{1}{\alpha}}\left(t+A_{2}\right)\right),
$$

assuming that $u_{2}^{-1}$ also exists.

One can learn more about the domain and range of $u_{2}$ as well as its inverse $u_{2}^{-1}$ in an analogous manner to how the same was investigated for $u_{1}$. Let $u_{2}$ be defined and continuously differentiable on a neighbourhood $N_{1}$ of $y_{0}$. The primitive of $u_{2}$ is

$$
x \mapsto\left(1-\frac{x^{\alpha}}{\alpha E}\right)^{-\frac{1}{\alpha}} .
$$

Using the local inverse function theorem once again if

$$
\left|y_{0}\right|<(\alpha E)^{\frac{1}{\alpha}}
$$

is true, then the inverse $u_{2}^{-1}$ exists for every point in the domain of $u_{2}$ such that it is continuously differentiable and defined on the image $u_{2}\left(N_{1}\right)=N_{2}$. Notice, the 
condition (B.19) coincides with (B.16). Hence (B.16) acts as the uniform condition for the existence of both the equation (B.15) and the inverse $u_{2}^{-1}$.

It is now clear that the conditions (B.10) and (B.16) are important for our solutions to be well-defined. To build on this we could use Theorem A.4 to extend them so that we also get absolute convergence for when $|q|=\left(\alpha^{*} E\right)^{\frac{1}{\alpha^{*}}}$ and $|p|=$ $(\alpha E)^{\frac{1}{\alpha}}$. However, having this extension would leave (B.13) and (B.18) as possibly undefined. Therefore, although we would gain a larger class of $q$ and $p$ such that the hypergeometric series converge, this extension does not allow the inverses of $u_{1}$ and $u_{2}$ to be well-defined. Hence we keep to the conditions $|q|<\left(\alpha^{*} E\right)^{\frac{1}{\alpha^{*}}}$ and $|p|<(\alpha E)^{\frac{1}{\alpha}}$.

Now that we have the general solutions, we can solve the initial value problem by incorporating the initial conditions $q\left(t_{0}\right)=q_{0}$ and $p\left(t_{0}\right)=p_{0}$. We will take the initial value of $t$ to be 0 .

Using $q(0)=q_{0}$, the general solution (B.11) appears as

$$
u_{1}^{-1}\left((\alpha E)^{\frac{1}{\alpha^{*}}} A_{1}\right)=q_{0},
$$

or rearranging for $A_{1}$,

$$
A_{1}=(\alpha E)^{\frac{-1}{\alpha^{*}}} u_{1}\left(q_{0}\right) .
$$

This gives the complete solution

$$
q(t)=u_{1}^{-1}\left((\alpha E)^{\frac{1}{\alpha^{*}}} t+u_{1}\left(q_{0}\right)\right) .
$$

Similarly using $p(0)=p_{0}$ the general solution (B.17) follows as

$$
u_{2}^{-1}\left(-\left(\alpha^{*} E\right)^{\frac{1}{\alpha}} A_{2}\right)=p_{0},
$$

or rearranging for $A_{2}$,

$$
A_{2}=-\left(\alpha^{*} E\right)^{\frac{-1}{\alpha}} u_{2}\left(p_{0}\right) .
$$

Therefore the complete solution for $p$ appears as

$$
p(t)=u_{2}^{-1}\left(-\left(\alpha^{*} E\right)^{\frac{1}{\alpha}} t+u_{2}\left(p_{0}\right)\right) .
$$

The above method may be repeated for each of the three remiaining cases of signs $q$ and $p$ may have. For example, when $q \geq 0$ and $p<0$, the system of differential equations (B.7) becomes

$$
\left\{\begin{array}{l}
\dot{q}=-\alpha^{\frac{1}{\alpha^{*}}}\left(E-\frac{1}{\alpha^{*}} q^{\alpha^{*}}\right)^{\frac{1}{\alpha^{*}}} \\
\dot{p}=-\alpha^{* \frac{1}{\alpha}}\left(E-\frac{1}{\alpha}(-p)^{\alpha}\right)^{\frac{1}{\alpha}}
\end{array}\right.
$$


This gives the solutions

$$
q(t)=u_{1}^{-1}\left(-(\alpha E)^{\frac{1}{\alpha^{*}}} t+u_{1}\left(q_{0}\right)\right)
$$

and

$$
p(t)=\tilde{u}_{2}^{-1}\left(-\left(\alpha^{*} E\right)^{\frac{1}{\alpha}} t+\tilde{u}_{2}\left(p_{0}\right)\right),
$$

where $u_{1}$ is as at (B.12), and $\tilde{u}_{2}$ is defined as

$$
\tilde{u}_{2}(p):=p_{2} F_{1}\left(\frac{1}{\alpha}, \frac{1}{\alpha} ; \frac{\alpha+1}{\alpha} ; \frac{(-p)^{\alpha}}{\alpha E}\right) .
$$

Comparing the above solutions to what was obtained when $q \geq 0$ and $p \geq 0$, we see that the only difference is the sign coefficient for certain expressions. For example there is an additional minus for the solution of $q$ at (B.23) when compared to solution of $q$ at (B.20). Similar observations may be made as the method is carried out for when $q<0$ and $p \geq 0$, and $q<0$ and $p<0$.

In collecting the four sets of solutions we would eventually have, we observe that whenever $q<0$ or $p<0$, the terms $-q$ or $-p$ may equivalently be written as $|q|$ or $|p|$ respectively. Using this we present the following solutions covering all four possible cases of signs that $q$ or $p$ may have.

We begin with the following system of differential equations who acts as a generalisation of the systems (B.7) and (B.22):

$$
\left\{\begin{array}{l}
\dot{q}= \pm \alpha^{\frac{1}{\alpha^{*}}}\left(E-\frac{1}{\alpha^{*}}|q|^{\alpha^{*}}\right)^{\frac{1}{\alpha^{*}}} \\
\dot{p}= \pm \alpha^{* \frac{1}{\alpha}}\left(E-\frac{1}{\alpha}|p|^{\alpha}\right)^{\frac{1}{\alpha}} .
\end{array}\right.
$$

Having used the initial conditions, the solutions for $q \in \mathbb{R}$ and $p \in \mathbb{R}$ follow as

$$
q(t)=f_{1}^{-1}\left( \pm(\alpha E)^{\frac{1}{\alpha^{*}}} t+f_{1}\left(q_{0}\right)\right)
$$

and

$$
p(t)=f_{2}^{-1}\left( \pm\left(\alpha^{*} E\right)^{\frac{1}{\alpha}} t+f_{2}\left(p_{0}\right)\right)
$$

where the functions $f_{1}$ and $f_{2}$ are defined as

$$
f_{1}(q):=q_{2} F_{1}\left(\frac{\alpha-1}{\alpha}, \frac{\alpha-1}{\alpha} ; \frac{2 \alpha-1}{\alpha} ; \frac{|q|^{\alpha^{*}}}{\alpha^{*} E}\right)
$$

and

$$
f_{2}(p):=p_{2} F_{1}\left(\frac{1}{\alpha}, \frac{1}{\alpha} ; \frac{\alpha+1}{\alpha} ; \frac{|p|^{\alpha}}{\alpha E}\right)
$$


respectively. The signs in each of the expressions above is determined by which of the four cases of possible signs for $q$ and $p$ is considered. Using the same investigations as before the solutions as well as the inverses $f_{1}^{-1}$ and $f_{2}^{-1}$ can be found to be welldefined whenever $|q|<\left(\alpha^{*} E\right)^{\frac{1}{\alpha^{*}}}$ and $|p|<(\alpha E)^{\frac{1}{\alpha}}$.

Now we solve Lagrange's equation. Calculating the partial Legendre transform of the Hamilton function (B.1) with respect to $p$, the corresponding Lagrange function follows as

$$
\mathcal{L}(q, \eta)=\frac{1}{\alpha^{*}}\left(|\eta|^{\alpha^{*}}-|q|^{\alpha^{*}}\right), \quad \alpha^{*}=\frac{\alpha}{\alpha-1},
$$

where for $q$ and $\eta$ fixed it holds that $\mathcal{L}(., \eta) \in C^{2}(\mathbb{R})$ and $\mathcal{L}(q,.) \in C^{2}(\mathbb{R})$. Lagrange's equation follows as

$$
\dot{\eta}|\eta|^{\alpha^{*}-2}=\frac{-q|q|^{\alpha^{*}-2}}{\left(\alpha^{*}-1\right)} .
$$

Recall it was set that $\dot{q}=\eta$ and so (B.29) can be rewritten as

$$
\ddot{q}|\dot{q}|^{\alpha^{*}-2}=\frac{-q|q|^{\alpha^{*}-2}}{\left(\alpha^{*}-1\right)} .
$$

Similar to the system (B.2), the absolute values make it difficult to solve (B.30). Hence we use a similar approach to what was done to solve (B.2): we choose to initially only solve (B.30) for when $q \geq 0$ and $\dot{q} \geq 0$. We then use the fact that the method may be carried out analogously for the remaining cases $q \geq 0$ and $\dot{q}<0$, $q<0$ and $\dot{q} \geq 0$ and $q<0$ and $\dot{q}<0$, to ultimately express a solution that collects all the cases together as was done with the Hamilton function.

Beginning with $q \geq 0$ and $\dot{q} \geq 0$, the Lagrange function (B.28) follows as

$$
\mathcal{L}(q, \dot{q})=\frac{1}{\alpha^{*}}\left(\dot{q}^{\alpha^{*}}-q^{\alpha^{*}}\right)
$$

with Lagrange's equation at (B.30) appearing as

$$
\ddot{q} \dot{q}^{\alpha^{*}-2}=-\frac{q^{\alpha^{*}-1}}{\left(\alpha^{*}-1\right)} .
$$

Recollecting Theorem 3.3.2 we know that E satisfies

$$
\frac{\partial \mathcal{L}}{\partial \dot{q}} \dot{q}-\mathcal{L}=E,
$$

or

$$
\frac{1}{\alpha} \dot{q}^{\alpha^{*}}+\frac{1}{\alpha^{*}} q^{\alpha^{*}}=E .
$$


Rearranging (B.34) then solves $\dot{q}$ to be

$$
\dot{q}=\alpha^{\frac{1}{\alpha^{*}}}\left(E-\frac{1}{\alpha^{*}} q^{\alpha^{*}}\right)^{\frac{1}{\alpha^{*}}},
$$

which is the first equation of (B.7). Hence solving for $q$ will follow as it did before with the Hamilton function. Substituting the solution we would gain for $q$ into equation (B.35) would then allow us to solve for $\dot{q}$.

As mentioned before this method may then be carried out for the remaining cases of sign for $q$ and $\dot{q}$. For example when $q \geq 0$ and $\dot{q}<0$ the Lagrange function (B.28) appears as

$$
\mathcal{L}(q, \dot{q})=\frac{1}{\alpha^{*}}\left((-\dot{q})^{\alpha^{*}}-q^{\alpha^{*}}\right)
$$

giving $\dot{q}$ to satisfy

$$
\dot{q}=-\alpha^{\frac{1}{\alpha^{*}}}\left(E-\frac{1}{\alpha^{*}} q^{\alpha^{*}}\right)^{\frac{1}{\alpha^{*}}} .
$$

Comparing (B.36) and (B.37) to the Lagrange function (B.31) and equation (B.35) respectively, we see that the only difference is the addition of a minus at certain places. A similar observation was made when comparing corresponding terms between different cases in regard to Hamilton's equations. In fact we may follow what was done before even further and do similarly with the remaining cases. In collecting all four cases together, we observe that whenever $\dot{q}<0$ we can write $|\dot{q}|$ for $-\dot{q}$. Using this we present the following solutions covering all four possible cases for signs that $q$ or $\dot{q}$ may have.

We begin with the following equation who acts as a generalisation of (B.35) and (B.37):

$$
\dot{q}= \pm \alpha^{\frac{1}{\alpha^{*}}}\left(E-\frac{1}{\alpha^{*}}|q|^{\alpha^{*}}\right)^{\frac{1}{\alpha^{*}}}
$$

which coincides with the first equation of (B.24) who was used when solving Hamilton's equations. Hence from this point onwards solving for $q$ follows as it did when looking at the Hamilton function. As before the sign at (B.38) is determined by which case of signs for $q$ and $\dot{q}$ is considered. Given a solution for $q$, we can find $\dot{q}$ by substituting this solution into (B.38).

Example B.2. We want to study the dynamics associated with $\mathcal{H}_{1, K} \in C^{1}(\mathbb{R} \backslash\{0\} \times$ $\mathbb{R})$ such that

$$
\mathcal{H}_{1, K}(q, p)=\frac{1}{\alpha}|p|^{\alpha}-\gamma_{1, \alpha}|q|^{\alpha-1}, \quad \gamma_{1, \alpha}<0 .
$$


Hamilton's equations follow as

$$
\left\{\begin{array}{l}
\dot{q}=p|p|^{\alpha-2}, \\
\dot{p}=(\alpha-1) \gamma_{1, \alpha} q|q|^{\alpha-3}
\end{array}\right.
$$

Note there is a concern for having singularities in (B.40). The full details were discussed in Example 4.2.1 of Section 4.2.

The absolute values present in the system of differential equations (B.40) makes it difficult to solve. We follow what was done in Example B.1 which required the solving of a system of differential equations with a similar problem, namely (B.2). We will exploit a symmetry property and consider the system for four different cases, when $q>0$ and $p \geq 0, q>0$ and $p<0, q<0$ and $p \geq 0$ and $q<0$ and $p<0$. Having solved (B.40) for $q>0$ and $p \geq 0$, the method may then be carried out analogously for the three remaining cases. As before we do not do the latter. However to close we present solutions that cover each possible case for the sign of $q$ and $p$.

We begin with when $q>0$ and $p \geq 0$, i.e. the Hamilton function (B.39) appears as

$$
\mathcal{H}_{1, K}(q, p)=\frac{1}{\alpha} p^{\alpha}-\gamma_{1, \alpha} q^{\alpha-1}
$$

We solve

$$
\left\{\begin{array}{l}
\dot{q}=p^{\alpha-1}, \\
\dot{p}=(\alpha-1) \gamma_{1, \alpha} q^{\alpha-2}, \quad q>0, p \geq 0 .
\end{array}\right.
$$

As before we set initial conditions to employ, namely $q\left(t_{0}\right)=q_{0}$ and $p\left(t_{0}\right)=p_{0}$. Once the general solutions have been found, these conditions will be included to obtain the complete solutions for the initial value problem.

We know from Theorem 3.3.1 that the Hamilton function has a constant value $E$ determined by the initial conditons, i.e.

$$
\mathcal{H}_{1, K}(q(t), p(t))=\mathcal{H}_{1, K}\left(q_{0}, p_{0}\right)=E .
$$

Rearranging (B.42) we can obtain further equations for $q$ and $p$ :

$$
q=\left(-\tilde{\gamma}_{1, \alpha}\right)^{\frac{-1}{\alpha-1}}\left(\alpha E-p^{\alpha}\right)^{\frac{1}{\alpha-1}}>0 \text { and } p=\left(\alpha E+\tilde{\gamma}_{1, \alpha} q^{\alpha-1}\right)^{\frac{1}{\alpha}}>0 .
$$

Substituting these expressions into (B.41) we obtain a new system of decoupled ODE's:

$$
\left\{\begin{array}{l}
\dot{q}=\left(\alpha E+\tilde{\gamma}_{1, \alpha} q^{\alpha-1}\right)^{\frac{\alpha-1}{\alpha}}, \\
\dot{p}=-\alpha^{\frac{2-\alpha}{\alpha-1}}(\alpha-1)\left(-\gamma_{1, \alpha}\right)^{\frac{1}{\alpha-1}}\left(\alpha E-p^{\alpha}\right)^{\frac{\alpha-2}{\alpha-1}} .
\end{array}\right.
$$

Using the method of separation of variables with the first equation of (B.44) we have

$$
\int\left(\alpha E+\tilde{\gamma}_{1, \alpha} q^{\alpha-1}\right)^{\frac{1-\alpha}{\alpha}} \mathrm{d} q=\int 1 \mathrm{~d} t
$$


Integrating gives

$$
\begin{aligned}
& \alpha^{\frac{1-3 \alpha}{\alpha}} E^{\frac{1-2 \alpha}{\alpha}}\left(\alpha^{2} E q_{2} F_{1}\left(\frac{1}{\alpha-1}, \frac{-1}{\alpha} ; \frac{\alpha}{\alpha-1} ; \frac{-\tilde{\gamma}_{1, \alpha} q^{\alpha-1}}{\alpha E}\right)\right. \\
& \left.-\tilde{\gamma}_{\alpha, 1} q^{\alpha}{ }_{2} F_{1}\left(\frac{\alpha-1}{\alpha}, \frac{\alpha}{\alpha-1} ; \frac{2 \alpha-1}{\alpha-1} ; \frac{-\tilde{\gamma}_{1, \alpha} q^{\alpha-1}}{\alpha E}\right)\right) \\
& =t+A_{1}
\end{aligned}
$$

where $A_{1}$ denotes the constant obtained through integration. Note that the term $q^{\alpha^{*}}$ is well-defined since we have taken the assumption that $q>0$.

We use Definition A.5 once again to confirm that the hypergeometric series contained in (B.46) converges for

$$
|q|<\left(\frac{E}{-\gamma_{1, \alpha}}\right)^{\frac{1}{\alpha-1}} .
$$

In fact (B.47) agrees with what is needed for the first equation of (B.44) to be welldefined.

Using (B.46) the general solution for $q$ follows as

$$
q(t)=v_{1}^{-1}\left(\alpha^{\frac{3 \alpha-1}{\alpha}} E^{\frac{2 \alpha-1}{\alpha}}\left(t+A_{1}\right)\right)
$$

where we define

$$
\begin{aligned}
v_{1}(q):= & \alpha^{2} E q_{2} F_{1}\left(\frac{1}{\alpha-1}, \frac{-1}{\alpha} ; \frac{\alpha}{\alpha-1} ; \frac{-\tilde{\gamma}_{1, \alpha} q^{\alpha-1}}{\alpha E}\right) \\
& -\tilde{\gamma}_{\alpha, 1} q^{\alpha}{ }_{2} F_{1}\left(\frac{\alpha-1}{\alpha}, \frac{\alpha}{\alpha-1} ; \frac{2 \alpha-1}{\alpha-1} ; \frac{-\tilde{\gamma}_{1, \alpha} q^{\alpha-1}}{\alpha E}\right) .
\end{aligned}
$$

In Example B.1, a similar function to $v_{1}$ was obtained whose domain, range and inverse were investigated. We do the same here following what was done before. Let $v_{1}$ be defined and continuously differentiable on a neighbourhood of $x_{0}$. Then using the local inverse function theorem whenever $v_{1}^{\prime}\left(x_{0}\right) \neq 0$, then there exists a neighbourhood of $x_{0}$, let this be $U_{1}$, such that $\left.v_{1}\right|_{U_{1}}$ has a continuously differentiable inverse $v_{1}^{-1}$ defined on the image $U_{2}=v_{1}\left(U_{1}\right)$.

Employing the fundamental theorem of calculus, it holds that

$$
\alpha^{\frac{3 \alpha-1}{\alpha}} E^{\frac{2 \alpha-1}{\alpha}} \int\left(\alpha E+\tilde{\gamma}_{1, \alpha} x^{\alpha-1}\right)^{\frac{1-\alpha}{\alpha}} \mathrm{d} x=v_{1}(x) .
$$

Therefore $v_{1}$ is the primitive of the mapping

$$
x \mapsto \alpha^{\frac{3 \alpha-1}{\alpha}} E^{\frac{2 \alpha-1}{\alpha}}\left(\alpha E+\tilde{\gamma}_{1, \alpha} x^{\alpha-1}\right)^{\frac{1-\alpha}{\alpha}} .
$$


Through this we see that $v_{1}^{\prime}\left(x_{0}\right) \neq 0$ is equivalent to

$$
\left|x_{0}\right|<\left(\frac{E}{-\gamma_{1, \alpha}}\right)^{\frac{1}{\alpha-1}} .
$$

Thus, under the condition (B.51) the inverse $v_{1}^{-1}$ exists for every point in the domain of $v_{1}$. This gives $v_{1}$ as a mapping from $U_{1}$ to $U_{2}$. Observe the condition (B.51) coincides with the inequality we needed at (B.47). Hence, (B.47) acts as a uniform condition for the existence of both the equation (B.46) and the inverse $v_{1}^{-1}$.

We now move on to the second equation in the system (B.44). Using the method of separation of variables we have

$$
-\frac{\alpha^{\frac{\alpha-2}{\alpha-1}}\left(-\gamma_{1, \alpha}\right)^{\frac{-1}{\alpha-1}}}{\alpha-1} \int\left(\alpha E-p^{\alpha}\right)^{\frac{2-\alpha}{\alpha-1}} \mathrm{~d} p=\int 1 \mathrm{~d} t .
$$

Integrating then gives

$$
-\frac{\left(-\gamma_{1, \alpha}\right)^{\frac{-1}{\alpha-1}} E^{\frac{2-\alpha}{\alpha-1}}}{\alpha-1} p_{2} F_{1}\left(\frac{\alpha-2}{\alpha-1}, \frac{1}{\alpha} ; \frac{\alpha+1}{\alpha} ; \frac{p^{\alpha}}{\alpha E}\right)=t+A_{2},
$$

$A_{2}$ a further constant of integration. Note the term $p^{\alpha}$ is always well-defined since we have taken the assumption that $p \geq 0$. Recollecting Definition A.5 one more time the hypergeometric series contained in (B.53) converges for

$$
|p|<(\alpha E)^{\frac{1}{\alpha}}
$$

Similar to what we had with (B.47), the inequality (B.54) agrees with what is needed for the second equation of (B.44) to be well-defined.

Defining a further continuously differentiable function $v_{2}$ :

$$
v_{2}(p):=p_{2} F_{1}\left(\frac{\alpha-2}{\alpha-1}, \frac{1}{\alpha} ; \frac{\alpha+1}{\alpha} ; \frac{p^{\alpha}}{\alpha E}\right),
$$

it holds that

$$
p(t)=v_{2}^{-1}\left(-\left(-\gamma_{1, \alpha}\right)^{\frac{1}{\alpha-1}}(\alpha-1) E^{\frac{\alpha-2}{\alpha-1}}\left(t+A_{2}\right)\right) .
$$

One can learn more about the domain, range and inverse of $v_{2}$ in an analogous manner to how this was done for $v_{1}$. The primitive of $v_{2}$ is

$$
x \mapsto(\alpha E)^{\frac{\alpha-2}{\alpha-1}}\left(\alpha E-x^{\alpha}\right)^{\frac{2-\alpha}{\alpha-1}} .
$$

Hence, assuming $v_{2}$ is defined and continuously differentiable on a neighbourhood $V_{1}$ of $y_{0}$, the condition we need is

$$
\left|y_{0}\right|<(\alpha E)^{\frac{1}{\alpha}} .
$$


Thus (B.57) gives that the inverse of $\left.v_{2}\right|_{V_{1}}, v_{2}^{-1}$, exists for every point in the domain of $v_{2}$ such that it is continuously differentiable and defined on the image $v_{2}\left(V_{1}\right)=$ $V_{2}$. Notice, the condition (B.57) coincides with the inequality we needed at (B.54). Hence, (B.54) in fact acts as the uniform condition for the existence of both the equation (B.53) and the inverse $v_{2}^{-1}$.

It is now clear that the conditions (B.47) and (B.54) are important for our solutions to be well-defined. To build on this we could try to use Theorem A.4 as we tried to do in Example B.1 to extend them. This would give absolute convergence for when $|q|=\left(\frac{E}{-\gamma_{1, \alpha}}\right)^{\frac{1}{\alpha-1}}$ and $|p|=(\alpha E)^{\frac{1}{\alpha}}$. However we encounter a similar problem to before. Having this extension would leave equation (B.50) and the second equation of (B.44) as possibly undefined. Therefore we keep to the conditions $|q|<\left(\frac{E}{-\gamma_{1, \alpha}}\right)^{\frac{1}{\alpha-1}}$ and $|p|<(\alpha E)^{\frac{1}{\alpha}}$.

We can now solve the initial value problem by incorporating the initial conditions $q\left(t_{0}\right)=q_{0}$ and $p\left(t_{0}\right)=p_{0}$. As before we will take the initial value of $t$ to be 0 .

Using $q(0)=q_{0}$, the general solution (B.48) appears as

$$
v_{1}^{-1}\left(\alpha^{\frac{3 \alpha-1}{\alpha}} E^{\frac{2 \alpha-1}{\alpha}} A_{1}\right)=q_{0}
$$

or rearranging for $A_{1}$,

$$
A_{1}=\alpha^{\frac{1-3 \alpha}{\alpha}} E^{\frac{1-2 \alpha}{\alpha}} v_{1}\left(q_{0}\right) .
$$

This gives the complete solution

$$
q(t)=v_{1}^{-1}\left(\alpha^{\frac{3 \alpha-1}{\alpha}} E^{\frac{2 \alpha-1}{\alpha}} t+v_{1}\left(q_{0}\right)\right) .
$$

Similarly using $p(0)=p_{0}$ the general solution (B.55) follows as

$$
v_{2}^{-1}\left(-\left(-\gamma_{1, \alpha}\right)^{\frac{1}{\alpha-1}}(\alpha-1) E^{\frac{\alpha-2}{\alpha-1}} A_{2}\right)=p_{0},
$$

or rearranging for $A_{2}$ gives

$$
A_{2}=-\frac{\left(-\gamma_{1, \alpha}\right)^{\frac{-1}{\alpha-1}} E^{\frac{2-\alpha}{\alpha-1}}}{\alpha-1} v_{2}\left(p_{0}\right) .
$$

Therefore the complete solution for $p$ appears as

$$
p(t)=v_{2}^{-1}\left(-\left(-\gamma_{1, \alpha}\right)^{\frac{1}{\alpha-1}}(\alpha-1) E^{\frac{\alpha-2}{\alpha-1}} t+v_{2}\left(p_{0}\right)\right) .
$$

The above method may be repeated for each of the three remiaining cases for the sign of $q$ and $p$. For example, when $q>0$ and $p<0$, the system of differential equations 
(B.44) becomes

$$
\left\{\begin{array}{l}
\dot{q}=-\left(\alpha E+\tilde{\gamma}_{1, \alpha} q^{\alpha-1}\right)^{\frac{\alpha-1}{\alpha}} \\
\dot{p}=-\alpha^{\frac{2-\alpha}{\alpha-1}}(\alpha-1)\left(-\gamma_{1, \alpha}\right)^{\frac{1}{\alpha-1}}\left(\alpha E-(-p)^{\alpha}\right)^{\frac{\alpha-2}{\alpha-1}}
\end{array}\right.
$$

The corresponding solutions then follow as

$$
q(t)=v_{1}^{-1}\left(-\alpha^{\frac{3 \alpha-1}{\alpha}} E^{\frac{2 \alpha-1}{\alpha}} t+v_{1}\left(q_{0}\right)\right)
$$

and

$$
p(t)=\tilde{v}_{2}^{-1}\left(-\left(-\gamma_{1, \alpha}\right)^{\frac{1}{\alpha-1}}(\alpha-1) E^{\frac{\alpha-2}{\alpha-1}} t+\tilde{v}_{2}\left(p_{0}\right)\right) .
$$

In the above $v_{1}$ is as at (B.49) and $\tilde{v}_{2}$ is defined as

$$
\tilde{v}_{2}(p):=p_{2} F_{1}\left(\frac{\alpha-2}{\alpha-1}, \frac{1}{\alpha} ; \frac{\alpha+1}{\alpha} ; \frac{(-p)^{\alpha}}{\alpha E}\right) .
$$

We now make a similar observation to what was made when comparing individual cases of $q$ and $p$ in Example B.1. We look back to the solutions (B.58) and (B.59) that were obtained for when $q>0$ and $p \geq 0$. In comparison to (B.61) and (B.62) above, the only difference is the sign coefficient for certain expressions. For example there is an additional minus for the solution of $q$ at (B.61) when compared to solution of $q$ at (B.58). As before similar observations may be made as the method is carried out for when $q<0$ and $p \geq 0$, and $q<0$ and $p<0$.

In collecting the four sets of solutions we would eventually have, we observe that whenever $q<0$ or $p<0$, the terms $-q$ or $-p$ may equivalently be written as $|q|$ or $|p|$ respectively. Using this we present the following solutions covering all four possible cases for signs that $q$ or $p$ may have.

The systems of differential equations (B.44) and (B.60) turn out to be specfiic cases of

$$
\left\{\begin{array}{l}
\dot{q}= \pm\left(\alpha E+\tilde{\gamma}_{1, \alpha}|q|^{\alpha-1}\right)^{\frac{1}{\alpha^{*}}} \\
\dot{p}= \pm(\alpha-1) \alpha^{\frac{2-\alpha}{\alpha-1}}\left(-\gamma_{1, \alpha}\right)^{\frac{1}{\alpha-1}}\left(\alpha E-|p|^{\alpha}\right)^{\frac{\alpha-2}{\alpha-1}}
\end{array}\right.
$$

Having used the initial conditions, the solutions then follow as

$$
q(t)=g_{1}^{-1}\left( \pm \alpha^{\frac{3 \alpha-1}{\alpha}} E^{\frac{2 \alpha-1}{\alpha}} t+g_{1}\left(q_{0}\right)\right)
$$

and

$$
p(t)=g_{2}^{-1}\left( \pm\left(-\gamma_{1, \alpha}\right)^{\frac{1}{\alpha-1}}(\alpha-1) E^{\frac{\alpha-2}{\alpha-1}} t+g_{2}\left(p_{0}\right)\right)
$$

where the functions $g_{1}$ and $g_{2}$ are defined as

$$
g_{1}(q):=\alpha^{2} E|q|_{2} F_{1}\left(\frac{1}{\alpha-1}, \frac{-1}{\alpha} ; \frac{\alpha}{\alpha-1} ; \frac{-\tilde{\gamma}_{1, \alpha}|q|^{\alpha-1}}{\alpha E}\right)
$$




$$
-\tilde{\gamma}_{1, \alpha}|q|^{\alpha}{ }_{2} F_{1}\left(\frac{\alpha-1}{\alpha}, \frac{\alpha}{\alpha-1} ; \frac{2 \alpha-1}{\alpha-1} ; \frac{-\tilde{\gamma}_{1, \alpha}|q|^{\alpha-1}}{\alpha E}\right)
$$

and

$$
g_{2}(p):=p_{2} F_{1}\left(\frac{\alpha-2}{\alpha-1}, \frac{1}{\alpha} ; \frac{\alpha+1}{\alpha} ; \frac{|p|^{\alpha}}{\alpha E}\right),
$$

respectively. The signs in each of the expressions above is determined by which of the four cases of possible signs for $q$ and $p$ is considered. The solutions as well as the inverses $g_{1}^{-1}$ and $g_{2}^{-1}$ can be found to be well-defined whenever $|q|<\left(\frac{E}{-\gamma_{1, \alpha}}\right)^{\frac{1}{\alpha-1}}$ and $|p|<(\alpha E)^{\frac{1}{\alpha}}$ as before.

Now we solve the corresponding Lagrange's equation. The corresponding Lagrange function for the Hamilton function (B.39) follows as

$$
\mathcal{L}_{1, K}(q, \eta)=\frac{1}{\alpha^{*}}|\eta|^{\alpha^{*}}+\gamma_{1, \alpha}|q|^{\alpha-1}, \quad \alpha^{*}=\frac{\alpha}{\alpha-1},
$$

where $\mathcal{L}_{1, K}(., \eta) \in C^{1}(\mathbb{R} \backslash\{0\})$ and $\mathcal{L}_{1, K}(q,.) \in C^{2}(\mathbb{R})$. Lagrange's equation follows as

$$
\dot{\eta}|\eta|^{\alpha^{*}-2}=\gamma_{1, \alpha}(\alpha-1)^{2} q|q|^{\alpha-3} .
$$

Recall it was set that $\dot{q}=\eta$ and so (B.66) may alternatively be rewritten as

$$
\ddot{q}|\dot{q}|^{\alpha^{*}-2}=\gamma_{1, \alpha}(\alpha-1)^{2} q|q|^{\alpha-3} .
$$

The absolute values make it once again difficult to solve the differential equation at (B.67). Hence we use a similar approach to what was done to solve (B.40): we choose to initially only solve (B.67) for when $q>0$ and $\dot{q} \geq 0$. We then use that the method may be carried out analogously for the remaining cases $q>0$ and $\dot{q}<0$, $q<0$ and $\dot{q} \geq 0$ and $q<0$ and $\dot{q}<0$ to collect all the cases together within an overall single set of solutions.

Starting with $q>0$ and $\dot{q} \geq 0$, the Lagrange function (B.65) follows as

$$
\mathcal{L}_{1, K}(q, \dot{q})=\frac{1}{\alpha^{*}} \dot{q}^{\alpha^{*}}+\gamma_{1, \alpha} q^{\alpha-1}
$$

with Lagrange's equation at (B.67) following as

$$
\ddot{q} \dot{q}^{\alpha^{*}-2}=\gamma_{1, \alpha}(\alpha-1)^{2} q^{\alpha-2} .
$$

Using Theorem 3.3.2 once again, we know that E satisfies

$$
\frac{\partial \mathcal{L}_{1, K}}{\partial \dot{q}} \dot{q}-\mathcal{L}_{1, K}=E
$$


or

$$
\frac{1}{\alpha} \dot{q}^{\alpha^{*}}-\gamma_{1, \alpha} q^{\alpha-1}=E .
$$

Rearranging (B.71) then gives $\dot{q}$ to be

$$
\dot{q}=\left(\alpha E+\tilde{\gamma}_{1, \alpha} q^{\alpha-1}\right)^{\frac{1}{\alpha^{*}}}
$$

which is the first equation of (B.44). Therefore solving for $q$ will follow as it did before with the Hamilton function. Substituting the solution we would gain for $q$ into equation (B.72) would then allow us to solve for $\dot{q}$.

This method may be carried out for the remaining cases of sign for $q$ and $\dot{q}$. For example when $q>0$ and $\dot{q}<0$ the Lagrange function (B.65) appears as

$$
\mathcal{L}_{1, K}(q, \dot{q})=\frac{1}{\alpha^{*}}(-\dot{q})^{\alpha^{*}}+\gamma_{1, \alpha} q^{\alpha-1}
$$

giving $\dot{q}$ to satisfy

$$
\dot{q}=-\left(\alpha E+\tilde{\gamma}_{1, \alpha} q^{\alpha-1}\right)^{\frac{1}{\alpha^{*}}} .
$$

If we compare the Lagange function (B.73) and equation (B.74) to the corresponding terms for when $q>0$ and $\dot{q} \geq 0$, namely equations (B.68) and (B.72) respectively, we see that the only difference is the addition of a minus at certain places. We noticed similar properties when comparing terms between different cases when looking at Hamilton's equations. In fact, writing $|\dot{q}|$ for $-\dot{q}$ for whenevrer $\dot{q}<0$, we may present a result covering all possible cases of sign for $q$ and $\dot{q}$.

Equations (B.72) and (B.74) are specific cases of

$$
\dot{q}= \pm\left(\alpha E+\tilde{\gamma}_{1, \alpha}|q|^{\alpha-1}\right)^{\frac{1}{\alpha^{*}}}
$$

which coincides with the first equation of (B.63) when solving Hamilton's equations. Hence from this point onwards solving for $q$ follows as it did when looking at the Hamilton function. As before the sign at (B.75) is determined by which case of signs for $q$ and $\dot{q}$ is considered. Given a solution for $q$, we can find $\dot{q}$ by substituting this solution into (B.75).

We now look at studying the two-dimenional extension on the previous two examples. Recall we choose to treat a general potential $V$ to cover both the Harmonic Oscillator and the Kepler problem.

Example B.3. The Hamilton function whose dynamics we want to study is $\mathcal{H} \in$ $C^{1}\left(\mathbb{R}^{4}\right)$ such that

$$
\mathcal{H}(q, p)=\frac{1}{\alpha}\|p\|^{\alpha}+V(q) .
$$


Hamilton's equations follow as

$$
\left\{\begin{array}{l}
\dot{q}_{j}=p_{j}\|p\|^{\alpha-2}, \\
\dot{p}_{j}=-V_{q_{j}}(q),
\end{array} \quad j=1,2 .\right.
$$

Solving the above system can be difficult in its current form. A useful change of variables is to polar coordinates, i.e. taking the Cartesian coordinates to be

$$
q_{1}=r \cos \vartheta \quad \text { and } \quad q_{2}=r \sin \vartheta
$$

where $r \geq 0$ and $\vartheta \in[0,2 \pi)$.

With the above equations, the Hamilton function (B.76) is equivalent to the following new function $\mathcal{H}_{2} \in C^{1}((0, \infty) \times[0,2 \pi) \times \mathbb{R} \backslash\{0\} \times \mathbb{R})$ :

$$
\mathcal{H}_{2}\left(r, \vartheta, p_{r}, p_{\vartheta}\right)=\frac{1}{\alpha}\left(p_{r}^{2}+\frac{p_{\vartheta}^{2}}{r^{2}}\right)^{\frac{\alpha}{2}}+V(r) .
$$

Hamilton's equations follow as:

$$
\begin{gathered}
\dot{r}=p_{r}\left(p_{r}^{2}+\frac{p_{\vartheta}^{2}}{r^{2}}\right)^{\frac{\alpha-2}{2}}, \\
\dot{\vartheta}=\frac{p_{\vartheta}}{r^{2}}\left(p_{r}^{2}+\frac{p_{\vartheta}^{2}}{r^{2}}\right)^{\frac{\alpha-2}{2}}, \\
\dot{p}_{r}=\frac{p_{\vartheta}^{2}}{r^{3}}\left(p_{r}^{2}+\frac{p_{\vartheta}^{2}}{r^{2}}\right)^{\frac{\alpha-2}{2}}-V_{r}(r)
\end{gathered}
$$

and

$$
\dot{p}_{\vartheta}=0
$$

implying that $p_{\vartheta}(t)=\kappa$ for all $t, \kappa$ being some constant.

We now make use of Section 3.3 and establish any conserved quantities we can exploit. We know that the Hamilton function (B.79) has a conserved value E determined by the initial values, i.e.

$$
\mathcal{H}_{2}\left(r(t), \vartheta(t), p_{r}(t), p_{\vartheta}(t)\right)=\mathcal{H}_{2}\left(r^{0}, \vartheta^{0}, p_{r}^{0}, p_{\vartheta}^{0}\right)=E>0 .
$$

Rearranging (B.82) and taking $p_{\vartheta}=\kappa$, we find $p_{r}$ to be

$$
p_{r}= \pm \sqrt{\alpha^{\frac{2}{\alpha}}(E-V(r))^{\frac{2}{\alpha}}-\frac{\kappa^{2}}{r^{2}}}
$$


Substituting this new value into (B.80) with $p_{\vartheta}=\kappa$ we have

$$
\dot{r}= \pm \sqrt{\alpha^{\frac{2}{\alpha}}(E-V(r))^{\frac{2}{\alpha}}-\frac{\kappa^{2}}{r^{2}}} \alpha^{\frac{\alpha-2}{\alpha}}(E-V(r))^{\frac{\alpha-2}{\alpha}} .
$$

Using separation of variables with (B.84) gives

$$
\gamma+t= \pm \int \frac{\alpha^{\frac{2-\alpha}{\alpha}} r(E-V(r))^{\frac{2-\alpha}{\alpha}}}{\sqrt{\alpha^{\frac{2}{\alpha}} r^{2}(E-V(r))^{\frac{2}{\alpha}}-\kappa^{2}}} \mathrm{~d} r
$$

for a constant of integration $\gamma$. When able to integrate the right-hand side of (B.85) explicitly, we would obtain an expression in terms of $r$. Rearranging this new equation would then solve $r$ in terms of $t$ and the constants $E, \kappa$ and $\gamma$. This would give the general solution for $r$. The constant $\gamma$ may then be eliminated using the initial conditions.

The solution for $r$ may then be used to solve $\vartheta$. Substituting (B.83) into (B.81) and taking $p_{\vartheta}=\kappa$ we have

$$
\dot{\vartheta}=\frac{\kappa \alpha^{\frac{\alpha-2}{\alpha}}}{r^{2}}(E-V(r))^{\frac{\alpha-2}{\alpha}} .
$$

We may then substitute our solution for $r$ from (B.85) into (B.86) to obtain a firstorder differential equation involving only $\vartheta$. This equation may then be integrated to find $\vartheta$ with a further constant of integration. This constant may then be eliminated as before using initial conditions to completely solve $\vartheta$.

The corresponding Lagrange function to (B.76) is

$$
\mathcal{L}(q, \eta)=\frac{1}{\alpha^{*}}\|\eta\|^{\alpha^{*}}-V(q)
$$

where $\mathcal{L}(., \eta) \in C^{1}\left(\mathbb{R}^{2}\right)$ and $\mathcal{L}(q,.) \in C^{2}\left(\mathbb{R}^{2}\right)$. Lagrange's equations in this case appear as

$$
\ddot{q}_{j}\|\dot{q}\|^{\alpha^{*}-2}+\left(\alpha^{*}-2\right) \dot{q}_{j}\|\dot{q}\|^{\alpha^{*}-4} \sum_{l=1}^{2} \dot{q}_{l} \ddot{q}_{l}=-V_{q_{j}}(q), \quad j=1, \ldots, n,
$$

which can be difficult to manage in the above form. As with the Hamilton function (B.76) we adopt polar coordinates. Using the transformation equations (B.78) the Lagrange function (B.87) can be rewritten as

$$
\mathcal{L}_{2}(r, \vartheta, \dot{r}, \dot{\vartheta})=\frac{1}{\alpha^{*}}\left(\dot{r}^{2}+r^{2} \dot{\vartheta}^{2}\right)^{\frac{\alpha^{*}}{2}}-V(r)
$$


where $\mathcal{L}_{2}(., ., \dot{r}, \dot{\vartheta}) \in C^{1}((0, \infty) \times[0,2 \pi))$ and $\mathcal{L}_{2}(r, \vartheta, .,.) \in C^{2}\left(\mathbb{R}^{2}\right)$. Lagrange's equations follow as

$$
\frac{\mathrm{d}}{\mathrm{d} t}\left(\dot{r}\left(\dot{r}^{2}+r^{2} \dot{\vartheta}^{2}\right)^{\frac{\alpha^{*}-2}{2}}\right)=r \dot{\vartheta}^{2}\left(\dot{r}^{2}+r^{2} \dot{\vartheta}^{2}\right)^{\frac{\alpha^{*}-2}{2}}-V_{r}(r)
$$

and

$$
\frac{\mathrm{d}}{\mathrm{d} t}\left(r^{2} \dot{\vartheta}\left(\dot{r}^{2}+r^{2} \dot{\vartheta}^{2}\right)^{\frac{\alpha^{*}-2}{2}}\right)=0
$$

implying that $p_{\vartheta}(t)=\kappa$ for all $t, \kappa$ being some constant. We recall from Theorem 3.3.2 that $E$ satisfies

$$
\frac{\partial \mathcal{L}_{2}}{\partial \dot{r}} \dot{r}+\frac{\partial \mathcal{L}_{2}}{\partial \dot{\vartheta}} \dot{\vartheta}-\mathcal{L}_{2}=E
$$

or

$$
\frac{1}{\alpha}\left(\dot{r}^{2}+r^{2} \dot{\vartheta}^{2}\right)^{\frac{\alpha^{*}}{2}}+V(r)=E
$$

Rearranging (B.90) we have for $\dot{r}^{2}$,

$$
\dot{r}^{2}=\alpha^{\frac{2}{\alpha^{*}}}(E-V(r))^{\frac{2}{\alpha^{*}}}-r^{2} \dot{\vartheta}^{2} .
$$

Similarly, rearranging (B.90) for $\dot{\vartheta}^{2}$ we have

$$
\dot{\vartheta}^{2}=\frac{1}{r^{2}}\left(\alpha^{\frac{2}{\alpha^{*}}}(E-V(r))^{\frac{2}{\alpha^{*}}}-\dot{r}^{2}\right) .
$$

Substituting (B.92) into (B.89) gives for $\dot{r}$

$$
\dot{r}= \pm \sqrt{\alpha^{\frac{2}{\alpha}}(E-V(r))^{\frac{2}{\alpha}}-\frac{\kappa^{2}}{r^{2}}} \alpha^{\frac{\alpha-2}{\alpha}}(E-V(r))^{\frac{\alpha-2}{\alpha}}
$$

which coincides with (B.84). Therefore we can use separation of variables once again to obtain the expression (B.85).

Substituting (B.91) into (B.89) gives for $\dot{\vartheta}$

$$
\dot{\vartheta}=\frac{\kappa \alpha^{\frac{\alpha-2}{\alpha}}}{r^{2}}(E-V(r))^{\frac{\alpha-2}{\alpha}}
$$

which coincides with (B.86). Hence the coordinates $r$ and $\vartheta$ may then be solved as they were when considering the Hamilton function.

To obtain the special cases of the Harmonic Oscillator and the Kepler problem we carry out the method as seen above and take $V(q)=\frac{1}{\alpha^{*}}\|q\|^{\alpha^{*}}$ and $V(q)=$ $-\gamma_{2, \alpha}\|q\|^{\alpha-2}$ respectively. The solutions obtained here would then coincide with what is seen at Examples 4.1.2 and 4.2.2. Note in the case of the Kepler problem we would again have to impose that $q \neq 0$ to avoid any singularities. 


\section{Bibliography}

[1] Andrews, G.E., Askey, R., Roy, R., Special Functions. Encyclopedia of Mathematics and its Applications, Vol. 71, Cambridge University Press, Cambridge 1999.

[2] Arnold, V.I., Mathematical Methods of Classical Mechanics, 2nd ed. Graduate Texts in Mathematics 60, Springer Verlag, New York 1989.

[3] Beals, R., Wong, R., Special Functions. A Graduate Text. Cambridge Studies in Advanced Mathematics 126, Cambridge University Press, Cambridge 2010.

[4] Berg, C., Forst, G., Potential Theory on Locally Compact Abelian Groups. Ergebnisse der Mathematik und ihrer Grenzgebiete, Bd. 87, Springer Verlag, Berlin 1975.

[5] Bertoin, J., Lévy Processes. Cambridge Tract in Mathematics, Vol. 121, Cambridge University Press, Cambridge 1996.

[6] Bogdan, K., Byczkowski, T., Kulczycki, T., Ryznar, M., Song, R., Vondraček, Z., Potential Analysis of Stable Processes and Its Extensions. In: Graczyk, P., Stos, A., (eds.), Lecture Notes in Mathematics, Vol. 1980, Sringer-Verlag, Berlin 2009.

[7] Böttcher, B., Schilling, R.L., Wang, J., Lévy-Type Processes: Construction, Approximation and Sample Path Properties. Lecture Notes in Mathematics, Vol. 2099, Springer Verlag, Berlin 2013.

[8] Bray, L.J., Investigations on Transition Densities of Certain Classes of Stochastic Processes. PhD-thesis, Swansea University, Swansea 2016.

[9] Bray, L.J., Jacob, N., Some considerations on the structure of transition densities of symmetric Lévy processes. Commun. Stoch. Anal., 10 (2016), 405-420.

[10] Buttazzo, G., Giaquinta, M., Hildebrandt, S., One-dimensional Variational Problems. An Introduction. Oxford Lecture Series in Mathematics and Its Applications 15, Oxford University Press, New York 1998. 
[11] Calin, O., Chang, D-C., Sub-Riemannian Geometry. General Theory and Examples. Cambridge University Press, Cambridge 2009.

[12] Carmona, R., Masters, W.C., Simon, B., Relativistic Schrödinger operators: Asymptotic behaviour of the eigenfunctions. J. Funct. Anal., 91 (1990), 117-142.

[13] Daubechies, I., An uncertainity principle for functions with generalized kinetic energy. Commun. Math. Phys., 90 (1983), 311-320.

[14] Daubechies, I., One electron molecules with relativistic kinetic energy: Properties of the discrete spectrum. Commun. Math. Phys., 94 (1984), 523-535.

[15] Daubechies, I., Lieb, E., One electron relativistic molecules with Coulomb interaction. Commun. Math. Phys., 90 (1983), 497-510.

[16] Demuth, M., Casteren, J. van., On spectral theory of selfadjoint Feller generators. Reviews Math. Phys., 1 (1989), 325-414.

[17] Demuth, M., Casteren, J. van., Stochastic Spectral Theory for Selfadjoint Feller Operators - A Functional Integration Approach. Probability and Its Applications, Birkhäuser Verlag, Basel 2000.

[18] DiBenedetto, E., Real Analysis. Birkhäuser Advanced Texts, Basler Lehrbücher, Birkhäuser, Boston 2002.

[19] Ekeland, I., Convexity Methods in Hamiltonian Mechanics. Ergebnisse der Mathematik und ihrer Grenzgebiete, 3. Folge, Bd. 19, Springer Verlag, Berlin 1990.

[20] Evans, K.P., Jacob, N., (to appear).

[21] Fefferman, C., Phong, D.H., Subelliptic eigenvalue problems. In: Beckner, W., et al. (eds.), Conference on Harmonic Analysis in Honor of Antoni Zygmund, Wadsworth, Pacific Grove (1983), 590-606.

[22] Fefferman, C., Sanchez-Calle, A., Fundamental solutions for second order elliptic operators. Ann. Math., 124 (1986), 247-272.

[23] Forsyth, A.R., A Treatise on Differential Equations, 6th ed. St Martin's Press, New York 1961.

[24] Fukushima, M., Oshima, Y., Takeda, M., Dirichlet Forms and Symmetric Markov Processes. de Gruyter Studies in Mathematics 19, Walter de Gruyter Verlag, Berlin 1994. 
[25] Gasper, G., Rahman, M., Basic Hypergeometric Series, 2nd ed. Encyclopedia of Mathematics and its Applications, Vol. 96, Cambridge University Press, Cambridge 2004.

[26] Goldstein, H., Poole, C., Safko, J., Classical Mechanics, 3rd ed. Pearson Education International, Upper Saddle River, N.J. 074582002.

[27] Gregory, R.D., Classical Mechanics. An Undergraduate Text. Cambridge University Press, Cambridge 2006.

[28] Grigor'yan, A., Heat Kernels and Analysis on Manifolds. Providence, RI: Amer. Math. Soc., MA: International Press, Boston 2009.

[29] Grigor'yan, A., Hu, J., Lau, K-S., Comparison inequalities for heat semigroups and heat kernels on metric measure spaces. J. Funct. Anal., 259 (2010), 26132641.

[30] Harris, J., Investigations on Metric Spaces Associated with Continuous Negative Definite Functions and Bounds for Transition Densities of Certain Lévy Processes. PhD-thesis, Swansea University, Swansea 2016.

[31] Harris, J., Jacob, N., Some thoughts and investigations on densities of oneparameter operator semi-groups. In: Trutnau, G., et al. (eds.), Stochastic Partial Differential Equations and Related Fields, Festschrift Michael Röckner, Springer Verlag, (to appear 2018).

[32] Heinonen, J., Lectures on Analysis on Metric Spaces. Springer-Verlag, New York 2001.

[33] Herbst, I.W., Sloan, A.D., Perturbation of translation invariant positivity preserving semigroups on $L^{2}\left(\mathbb{R}^{n}\right)$. Trans. Amer. Math. Soc., 236 (1978), 325-360.

[34] Hoh, W., The martingale problem for a class of pseudo differential operators. Math. Ann., 300 (1994), 121-147.

[35] Hoh, W., A symbolic calculus for pseudo differential operators generating Feller semigroups. Osaka J. Math., 35 (1998), 798-820.

[36] Hoh, W., Pseudo Differential Operators Generating Markov Processes. Habilitationsschrift, Universitäet Bielefeld, Bielefeld 1998.

[37] Hörmander, L., Hypoelliptic second order differential equations. Acta Math., 119 (1967), 147-171. 
[38] Hörmander, L., The Analysis of Linear Partial Differential Operators I. Die Grundlehren der Mathematischen Wissenschaften, Vol. 256, Springer, Berlin 1983.

[39] Hörmander, L., The Analysis of Linear Partial Differential Operators II. Die Grundlehren der Mathematischen Wissenschaften, Vol. 257, Springer, Berlin 1983.

[40] Hörmander, L., The Analysis of Linear Partial Differential Operators III. Die Grundlehren der Mathematischen Wissenschaften, Vol. 274, Springer, Berlin 1985.

[41] Hörmander, L., The Analysis of Linear Partial Differential Operators IV. Die Grundlehren der Mathematischen Wissenschaften, Vol. 275, Springer, Berlin 1985.

[42] Ivrii, V.Y., Microlocal Analysis and Precise Spectral Asymptotics. Springer Verlag, Berlin 1998.

[43] Jacob, N., Pseudo-Differential Operators and Markov Processes. Vol.I: Fourier Analysis and Semigroups. Imperial College Press, London 2001.

[44] Jacob, N., Pseudo-Differential Operators and Markov Processes. Vol.II: Generators and Their Potential Theory. Imperial College Press, London 2002.

[45] Jacob, N., Pseudo-Differential Operators and Markov Processes. Vol.III: Markov Processes and Applications. Imperial College Press, London 2005.

[46] Jacob, N., Evans, K.P., A Course in Analysis Vol. I: Introductory Calculus, Analysis of Functions of One Real Variable. World Scientific Publishing, Singapore 2016.

[47] Jacob, N., Evans, K.P., A Course in Analysis. Vol. II: Differentiation and Integration of Functions of Several Variables, Vector Calculus. World Scientific Publishing, Singapore 2016.

[48] Jacob, N., Knopova, V., Landwehr, S., Schilling, R.L., A geometric interpretation of the transition density of a symmetric Lévy process. Sci China Math., 55 (2012), 1099-1126.

[49] Jacob, N., Rhind, E., Aspects of micro-local analysis and geometry in the study of Lévy-type generators. In: Witt, I., et al. (eds.), Dynamical Methods in Open Quantum Systems, Springer Verlag, (to appear 2018). 
[50] Knopova, V., Schilling, R.L., A note on the existence of transition probability densities for Lévy processes. Forum Math., 25 (2013), 125-149.

[51] Landau, L.D., Lifshitz, E.M., Mechanics and Electrodynamics, 1st ed. A Shorter Course of Theoretical Physics, Vol. 1, Pergamon Press, Oxford 1972.

[52] Landwehr, S., On the Geometry Related to Jump Processes. PhD-thesis, Swansea University, Swansea 2010.

[53] Maslov, V.P., Nonstandard characteristics in asymptotical problems. Proceedings of the International Congress of Mathematicians, Vol. 1, (Warsaw, 1983), PWN, Warsaw, (1984), 139-183.

[54] Nagel, A., Stein, E.M., Wainger, S., Balls and metrics defined by vector fields I: Basic properties. Acta Math., 155 (1985), 103-147.

[55] Rothschild, L., Stein, E.M., Hypoelliptic differential operators and nilpotent groups. Acta Math., 137 (1976), 247-320.

[56] Sato, K., Lévy Processes and Infinitely Divisible Distributions. Cambridge Studies in Advanced Mathematics, Vol. 68, Cambridge University Press, Cambridge 1999 .

[57] Schilling, R.L., On Feller processes with sample paths in Besov spaces. Math. Ann., 309 (1997), 663-675.

[58] Schilling, R.L., Feller processes generated by pseudo-differential operators: On the Hausdorff dimension of their sample paths. J. Theor. Probab., 11 (1998), 303-330.

[59] Schilling, R.L., Growth and Hölder conditions for the sample paths of Feller processes. Probab. Theory Relat. Fields., 112 (1998), 565-611.

[60] Schilling, R.L., Song, R., Vondraček, Z., Bernstein Functions. Theory and Applications. de Gruyter Studies in Mathematics 37, Walter de Gruyter, Berlin 2010 .

[61] Shubin, M.A., Pseudodifferential Operators and Spectral Theory. Transl. from the Russian, Springer Verlag, Berlin 1987.

[62] Simon, B., Convexity: An Analytic Viewpoint. Cambridge Tracts in Mathematics 187, Cambridge University Press, Cambridge 2011.

[63] Strichartz, R.S., Sub-Riemannian geometry. J. Differential Geom., 24 (1986), 221-263. 
[64] Strichartz, R.S., The Way of Analysis, rev.ed. Jones and Bartlett Publishers, Inc., Sudbury, MA 2000.

[65] Sturm, K-T., Analysis on local Dirichlet spaces. I. Recurrence, conservativeness and $L^{p}$-Liouville properties. J. Reine Angew. Math., 456 (1994), 173-196.

[66] Sturm, K-T., Analysis on local Dirichlet spaces. II. Upper Gaussian estimates for the fundamental solutions of parabolic equations. Osaka J. Math., 32 (1995), $275-312$.

[67] Sturm, K-T., Analysis on local Dirichlet spaces. III. The parabolic Harnack inequality. J. Math. Pures Appl., (9) 75 (1996), 273-297.

[68] Whittaker, E.T., Watson, G.N., A Course of Modern Analysis. An Introduction to the General Theory of Infinite Processes and of Analytic Functions; with an Account of the Principal Transcendental Functions, 4th ed. Cambridge University Press, Cambridge 1952. 